\title{
Simulação computacional adaptativa de escoamentos bifásicos viscoelásticos
}

\author{
Catalina Maria Rúa Alvarez
}

TESE APRESENTADA

$\mathrm{AO}$

Instituto DE MATEMÁTiCA E EstatísticA

DA

Universidade DE SÃo PAUlo

PARA

OBTENÇÃO DO TÍTULO

$\mathrm{DE}$

DOUTOR EM CIÊNCIAS

Programa: Matemática Aplicada

Orientador: Prof. Dr. Alexandre Megiorin Roma

Co-orientador: Prof. Dr. Hector D. Ceniceros

Durante o desenvolvimento deste trabalho o autor recebeu auxílio financeiro do $\mathrm{CNPq}_{\text {e da Petrobras. }}$

São Paulo, Julho de 2013. 


\title{
Simulação computacional adaptativa de escoamentos bifásicos viscoelásticos
}

\author{
Esta tese contém as correções e alterações \\ sugeridas pela Comissão Julgadora durante a defesa \\ realizada por Catalina Maria Rúa Alvarez em 28/05/2013. \\ O original encontra-se disponível no Instituto de \\ Matemática e Estatística da Universidade de São Paulo.
}

Comissão Julgadora:

- Prof. Dr. Alexandre Megiorin Roma (orientador) - IME-USP

- Prof. Dr. Luis Carlos Castro Santos - IME-USP

- Prof. Dr. Antonio Castelo Filho - ICMC-USP

- Prof. Dr. Rudimar Luiz Nós - UTFPE

- Prof. Dr. Aristeu da Silveira Neto - UFU-FEMEC 
"Para los amores de mi vida que nunca dejaron de creer en mi... A ustedes, John y Simon." 


\section{Agradecimentos}

Ao professor Alexandre, por toda sua ajuda, ensinamentos, orientação e paciência; é uma pessoa que admiro demasiado e a quem agradeço a conclusão desta tese e de minha parte espero receber inúmeras vezes a ele e à professora Angela no frio de Pasto para continuar esta e outras pesquisas. Ao professor Hector, por apresentar o problema, por toda sua disposição e por me receber junto com sua esposa Rosana na University of California Santa Barbara.

Ao Rudimar e à Millena, sem o trabalho de vocês eu não teria tido a oportunidade de trabalhar com estes grandes grupos de pesquisa FEMEC-UFU, ICMC-USP e IME-USP; toda sua ajuda e sugestões foi muito valiosa para mim. Aos membros da banca, pela paciência e leitura desta tese; suas correções enriqueceram este trabalho. Ao Cassio Oishi por compartilhar futuras aplicações e pesquisas que podem ser realizadas com este trabalho.

A toda minha família, ela é tão grande que tenho medo de esquecer de alguém, porque também considero meus grandes amigos como parte de minha família (mi mamá, mi papá, mis hermanos, mis sobrinos, mis suegros, mis cunhadas, mis tios y primos, mis amigos en Puerto Rico, para quienes estaban allí y ahora viven en otro lugar; para minha Pri Pri e o Alvaro, à Rita e o João, à Noely e o Pedro, os amigos do STAR, Eliza, Holguin y mis grandes amigos Aleja y Javi), em todo momento me acompanhando física ou emocionalmente, toda sua ajuda tem sido muito valiosa para mim.

Para as pessoas que completam minha alma, meu filho e meu esposo, agradeço sua espera e confiança, este trabalho é de vocês, não tenho palavras, "ustedes lo saben todo".

Aos colegas do LabMAP, do IME-USP e de trabalho com o professor Alexandre (Fabio Fonseca, Felipe Nunes e Wellington), os administradores do LabMAP, especialmente ao Marcello Souza. Ao pessoal do MFLab da Universidade Federal de Uberlândia e aos professores do ICMC-USP; com eles aprendi muitas coisas novas nas reuniões do projeto. Um agradecimento especial para o Ricardo Serfaty e para o professor Aristeu Silvera Neto que permitiram minha participação no projeto e deram seu apoio em parte desta pesquisa.

Agradeço a todos os funcionários do IME-USP, audiovisuais, secretárias do departamento do MAP, da contabilidade e à CPG. Ao IME, pelo auxílio financeiro na participação de eventos. Ao CNPq e à Petrobras pelo suporte financeiro durante a realização deste trabalho. 


\section{Resumo}

Rúa Alvarez, C.M. Simulação computacional adaptativa de escoamentos bifásicos viscoelásticos. 2013. Tese (Doutorado) - Instituto de Matemática e Estatística, Universidade de São Paulo, 2013.

A simulação computacional de escoamentos incompressíveis multifásicos tem avançado continuamente e é uma área extremamente importante em Dinâmica de Fluidos Computacional (DFC) por suas várias aplicações na indústria, em medicina e em biologia, apenas para citar alguns exemplos. Apresentamos modelos matemáticos e métodos numéricos tendo em vista simulações computacionais de fluidos bifásicos newtonianos e viscoelásticos (não newtonianos), em seus regimes transiente e estacionário de escoamento. Os ingredientes principais requeridos são o Modelo de Um Fluido e o Método da Fronteira Imersa em malhas adaptativas, usados em conjunto com os métodos da Projeção de Chorin-Temam e de Uzawa.

Tais metodologias são obtidas a partir de equações a derivadas parciais simples as quais, naturalmente, são resolvidas em malhas adaptativas empregando métodos multinível-multigrid. Em certas ocasiões, entretanto, para escoamentos modelados pelas equações de Navier-Stokes (e.g. em problemas onde temos altos saltos de massa específica), tem-se problemas de convergência no escopo destes métodos. Além disso, no caso de escoamentos estacionários, resolver as equações de Stokes em sua forma discreta por tais métodos não é uma tarefa fácil. Verificamos que zeros na diagonal do sistema linear resultante impedem que métodos de relaxação usuais sejam empregados.

As dificuldades mencionadas acima motivaram-nos a pesquisar por, a propor e a desenvolver alternativas à metodologia multinível-multigrid. No presente trabalho, propomos métodos para obter explicitamente as matrizes que representam os sistemas lineares oriundos da discretização daquelas equações a derivadas parciais simples que são a base dos métodos de Projeção e de Uzawa. Ter em mãos estas representações matriciais é vantajoso pois com elas podemos caracterizar tais sistemas lineares em termos das propriedades de seus raios espectrais, suas definições e simetria. Muito pouco (ou nada) se sabe efetivamente sobre estes sistemas lineares associados a discretizações em malhas compostas bloco-estruturadas. É importante salientarmos que, além disso, ganhamos acesso ao uso de bibliotecas numéricas externas, como o PETSc, com seus pré-condicionadores e métodos numéricos, seriais e paralelos, para resolver sistemas lineares.

Infraestrutura para nossos desenvolvimentos foi propiciada pelo código denominado "AMR2D", um código doméstico para problemas em DFC que vem sendo cuidado ao longo dos anos pelos grupos de pesquisa em DFC do IME-USP e da FEMEC-UFU. Estendemos este código, adicionando módulos para escoamentos viscoelásticos e para escoamentos estacionários modelados pelas equações de Stokes. Além disso, melhoramos de maneira notável as rotinas de cálculo de valores fantasmas. Tais melhorias permitiram a implementação do Método dos Gradientes Bi-Conjugados, baseada em visitas retalho-a-retalho e varreduras da estrutura hierárquica nível-a-nível, essencial à 
implementação do Método de Uzawa.

Palavras-chave: Escoamentos bifásicos, Método de Uzawa, Método da Projeção, refinamento adaptativo de malhas, fluido não newtoniano, baixo número de Reynolds. 


\section{Abstract}

Rúa Alvarez, C.M. Adaptive computational simulation of two-phase viscoelastic flows. 2013. Thesis (Ph.D.)- Institute of Mathematics and Statistics, University of São Paulo, 2013.

Numerical simulation of incompressible multiphase flows has continuously of advanced and is an extremely important area in Computational Fluid Dynamics (CFD) because its several applications in industry, in medicine, and in biology, just to mention a few of them. We present mathematical models and numerical methods having in sight the computational simulation of two-phase Newtonian and viscoelastic fluids (non-Newtonian fluids), in the transient and stationary flow regimes. The main ingredients required are the One-fluid Model and the Immersed Boundary Method on dynamic, adaptive meshes, in concert with Chorin-Temam Projection and the Uzawa methods.

These methodologies are built from simple linear partial differential equations which, most naturally, are solved on adaptive grids employing mutilevel-multigrid methods. On certain occasions, however, for transient flows modeled by the Navier-Stokes equations (e.g. in problems where we have high density jumps), one has convergence problems within the scope of these methods. Also, in the case of stationary flows, solving the discrete Stokes equations by those methods represents no straight forward task. It turns out that zeros in the diagonal of the resulting linear systems coming from the discrete equations prevent the usual relaxation methods from being used.

Those difficulties, mentioned above, motivated us to search for, to propose, and to develop alternatives to the multilevel-multigrid methodology. In the present work, we propose methods to explicitly obtain the matrices that represent the linear systems arising from the discretization of those simple linear partial differential equations which form the basis of the Projection and Uzawa methods. Possessing these matrix representations is on our advantage to perform a characterization of those linear systems in terms of their spectral, definition, and symmetry properties. Very little is known about those for adaptive mesh discretizations. We highlight also that we gain access to the use of external numerical libraries, such as PETSc, with their preconditioners and numerical methods, both in serial and parallel versions, to solve linear systems.

Infrastructure for our developments was offered by the code named "AMR2D" - an in-house CFD code, nurtured through the years by IME-USP and FEMEC-UFU CFD research groups. We were able to extend that code by adding a viscoelastic and a stationary Stokes solver modules, and improving remarkably the patchwise-based algorithm for computing ghost values. Those improvements proved to be essential to allow for the implementation of a patchwise Bi-Conjugate Gradient Method which "powers" Uzawa Method.

Keywords: Two-phase flows, Uzawa Method, Projection Method, adaptive mesh refinement, nonNewtonian fluid, low Reynolds number. 


\section{Sumário}

Lista de Abreviaturas xiii

Lista de Símbolos $\quad$ XV

Lista de Figuras $\quad$ xvii

Lista de Tabelas $\quad$ xix

Lista de Algoritmos $\quad$ xxi

1 Introdução $\quad \mathbf{1}$

1.1 Motivação científica . . . . . . . . . . . . . . . . . . . . 1

1.2 Metodologia e revisão da literatura . . . . . . . . . . . . . . . . . . . . . . . . . . . . . . . .

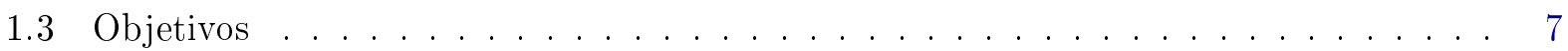

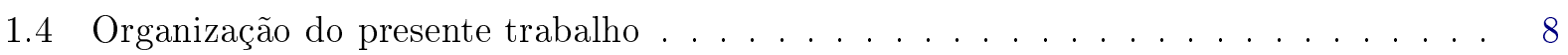

2 Modelos Matemáticos $\quad 11$

2.1 Escoamento incompressível de um fluido bifásico . . . . . . . . . . . . . . . 11

2.2 Dinâmica da interface de separação . . . . . . . . . . . . . . . . . . . . . . 14

2.3 Modelagem matemática da fase viscoelástica . . . . . . . . . . . . . . . . . 16

2.3.1 Detalhamento do Modelo Oldroyd-B . . . . . . . . . . . . . . . . 17

2.3.2 Detalhamento do Modelo FENE-P . . . . . . . . . . . . . . . . . . . 18

2.4 Adimensionalização do modelo matemático . . . . . . . . . . . . . . . . . . 20

2.5 Modelos matemáticos resultantes . . . . . . . . . . . . . . . . . . . . . 21

2.5.1 Escoamentos incompressíveis transientes de fluidos bifásicos . . . . . . . . . 21

2.5.2 Força interfacial . . . . . . . . . . . . . . . . . . . . . 21

2.5.3 Tensor viscoelástico . . . . . . . . . . . . . . . . . . . . . . . . . 22

2.5.4 Escoamentos incompressíveis estacionários de fluidos bifásicos . . . . . . . . 22

3 Metodologia numérica: discretizações no tempo e no espaço (malha uniforme) 23

3.1 Discretização no espaço: malhas uniformes . . . . . . . . . . . . . . . . . . . . 24

3.1.1 Domínio euleriano . . . . . . . . . . . . . . . . . . . . . 24

3.1 .2 Domínio lagrangiano . . . . . . . . . . . . . . . . 26

3.1 .3 Função indicadora de fluido . . . . . . . . . . . . . . . . . . . . 27

3.2 Escoamentos incompressíveis transientes . . . . . . . . . . . . . . . . 29

3.2.1 Método da Projeção de Chorin-Temam . . . . . . . . . . . . . . . . . . . 29 
3.2.2 Discretização no tempo: métodos implícitos-explícitos . . . . . . . . . . . . 30

3.2.3 Resumo da discretização do modelo transiente . . . . . . . . . . . . . . . . . 32

3.3 Discretização das equações lagrangianas . . . . . . . . . . . . . . . . . . 33

3.3.1 Discretização para a equação de movimento da interface . . . . . . . . . . . . . 34

3.3 .2 Discretização da força lagrangiana . . . . . . . . . . . . . . . . . . 35

3.4 Discretização do tensor viscoelástico . . . . . . . . . . . . . . . . . 36

3.4.1 Discretização do termo advectivo . . . . . . . . . . . . . . . . 36

3.4 .2 Modelo Oldroyd-B . . . . . . . . . . . . . . . . . . . . . . . . 40

3.4 .3 Modelo FENE-P . . . . . . . . . . . . . . . . . . . . . . . . 41

3.5 Escoamentos incompressíveis estacionários . . . . . . . . . . . . . . . 42

3.5.1 Comentários sobre a discretização da equação de Stokes em um fluido viscoelástico . . . . . . . . . . . . . . . . . . 45

3.6 Sumário da metodologia numérica f . . . . . . . . . . . . . . . . . 46

3.6.1 Escoamentos incompressíveis transientes de fluidos bifásicos . . . . . . . . . . 47

3.6 .2 Força interfacial . . . . . . . . . . . . . . . . . . . . . 47

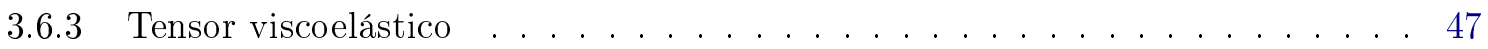

3.6.4 Escoamentos incompressíveis estacionários de fluidos bifásicos . . . . . . . . . 48

4 Metodologia numérica: AMR e representação matricial $\quad 51$

4.1 Domínio euleriano: malha composta . . . . . . . . . . . . . . . . . 52

4.1 .1 Definição da malha composta . . . . . . . . . . . . . . . . . 54

4.1.2 Células fantasmas e seus valores . . . . . . . . . . . . . . . . 55

4.1.3 Discretização dos operadores diferenciais na malha composta . . . . . . . . . 59

4.2 Estrutura matricial . . . . . . . . . . . . . . . . . . . . . 60

4.2 .1 Mapeamento de células e enumeração . . . . . . . . . . . . . . . . 63

4.2.2 Representação matricial de células fantasmas . . . . . . . . . . . . . . 68

4.2 .3 Representação matricial das discretizações . . . . . . . . . . . . . . . . . . 71

4.3 SAMR Método de Uzawa com BiCG-Stab (versão matricial) . . . . . . . . . . . . . . 72

5 Resultados numéricos $\quad \mathbf{7 5}$

5.1 Normas e análise da convergência numérica em malhas uniformes e em malhas com-

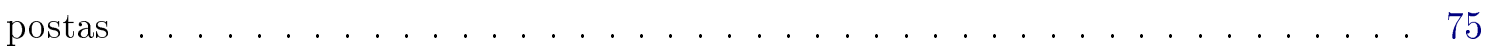

5.2 Verificação do cálculo matricial de valores fantasmas . . . . . . . . . . . . . 77

5.2.1 Resultados do cálculo matricial de valores fantasmas . . . . . . . . . . . . . 79

5.2.2 Tempo computacional do cálculo matricial de valores fantasmas . . . . . . . . 81

5.3 Resolução matricial de equações diferenciais parciais . . . . . . . . . . . . . . . 85

5.3.1 Primeiro problema-modelo: equação elíptica para variáveis centradas . . . . . 87

5.3.2 Segundo problema-modelo: equação elíptica para variáveis nas arestas . . . . 102

5.3.3 Terceiro problema-modelo: equação parabólica para variáveis nas arestas . . . 105

5.4 Verificação do tensor viscoelástico . . . . . . . . . . . . . . . . . . . . . . . 110

5.4 .1 Verificação do termo de advecção com ENO3 . . . . . . . . . . . . . . . 110

5.4 .2 Verificação do Modelo Oldroyd-B . . . . . . . . . . . . . . . . . . . . . . 113

5.4 .3 Verificação do Modelo FENE-P . . . . . . . . . . . . . . . . . . . . . . . 114

5.5 Verificação das equações de Navier-Stokes (escoamento incompressível transiente) . 115 
5.5.1 Fluido newtoniano monofásico . . . . . . . . . . . . . . . . . 115

5.5.2 Fluido não newtoniano monofásico . . . . . . . . . . . . . . . . . . 116

5.6 Verificação das equações de Stokes (escoamento incompressível estacionário) . . . . . 117

5.6 .1 Fluido newtoniano monofásico . . . . . . . . . . . . . . . . . . 117

5.6 .2 Fluido newtoniano bifásico f . . . . . . . . . . . . . . . . . . . . 119

5.6.3 Fluido não newtoniano monofásico . . . . . . . . . . . . . . . . . . . . . . 119

6 Conclusão e perspectivas futuras $\quad 123$

$\begin{array}{lr}\text { A Adimensionalização } & 127\end{array}$

B Mapa de visibilidade para variáveis definidas no meio das arestas 131

$\begin{array}{lll}\text { C Enumeração para variáveis definidas no meio das arestas } & 137\end{array}$

$\begin{array}{ll}\text { Referências Bibliográficas } & 141\end{array}$

$\begin{array}{lr}\text { Índice Remissivo } & 149\end{array}$ 


\section{Lista de Abreviaturas}

$\begin{array}{ll}\text { AMR } & \text { Adaptive Mesh Refinement } \\ \text { AMR2D } & \text { Código para o caso bidimensional da técnica AMR } \\ \text { AMR3D } & \text { Código para o caso tridimensional da técnica AMR } \\ \text { BiCG-Stab } & \text { Método do gradiente biconjugado estabilizado } \\ \text { CG } & \text { Método do Gradiente Conjugado } \\ \text { CSR } & \text { Compressed Sparse Rows } \\ \text { ENO } & \text { Esquema Essential Non-Oscillatory } \\ \text { ENO3 } & \text { Método ENO de terceira ordem } \\ \text { FENE } & \text { Finite Extendible Nonlinear Elastic } \\ \text { FENE-P } & \text { Aproximação do Modelo FENE proposta por Peterlin } \\ \text { GMRES } & \text { Método dos Resíduos Minimos Generalizados } \\ \text { ILU } & \text { Factorização LU incompleta } \\ \text { MMG } & \text { Método multinível-multigrid } \\ \text { NS } & \text { Equações de Navier-Stokes } \\ \text { Oldroyd-B } & \text { Modelo Oldroyd-B } \\ \text { PETSc } & \text { Portable, Extensible Toolkit for Scientific Computation } \\ \text { BCGS } & \text { Siglas no PETSc para o método do gradiente biconjugado estabilizado } \\ \text { SAMR } & \text { Structured Adaptive Mesh Refinement } \\ \text { SAMRAI } & \text { Structured Adaptive Mesh Refinement Infrastructure } \\ \text { SIMPLE } & \text { Semi-Implicit Method for Pressure Linked } \\ \text { SIMPLER } & \text { a SIMPLE Revised algorithm } \\ \text { RK2 } & \text { Método Runge-Kutta de segunda ordem } \\ \text { TVD } & \text { Total Variation Diminishing } \\ \text { Uzawa } & \text { Método de Uzawa }\end{array}$




\section{Lista de Símbolos}

u Campo de velocidade

$p \quad$ Campo de pressão

$\rho \quad$ Massa específica do fluido

$\mu \quad$ Viscosidade dinâmica

S Tensor de tensões produzido por macromoléculas

f Forças externas que atuam no fluido

$\alpha_{p} \quad$ Constante que controla se o fluido é puramente neutoniano ou não

$\Omega \quad$ Domínio euleriano

$\Gamma \quad$ Domínio lagrangiano

$\sigma \quad$ Tensão superficial

$\rho^{-} \quad$ Massa específica da fase dispersa

$\mu^{-} \quad$ Viscosidade dinâmica da fase dispersa

$\rho^{+} \quad$ Massa específica da fase contínua

$\mu^{+} \quad$ Viscosidade dinâmica da fase contínua

$\alpha \quad$ Parâmetro lagrangiano

$\kappa_{e} \quad$ Curvatura euleriana

$H$ Função de Heaviside

$\mathbf{f}_{s} \quad$ Formulação clássica para a força interfacial

$\mathbf{f}_{e} \quad$ Formulação euleriana para a força interfacial

$\mathbf{f}_{h} \quad$ Formulação híbrida para a força interfacial

$\mathbf{S}_{p} \quad$ Tensor viscoelástico

$\tau_{p} \quad$ Escala do tempo para a relaxação do polímero

$\mathbf{S}_{p}^{\nabla} \quad$ Derivada convectada superior

$\mathbf{C}^{(0)}$ Valor inicial do tensor $\mathbf{C}$

$\mathbf{S}_{p}^{(0)} \quad$ Valor inicial do tensor $\mathbf{S}$

$L_{c} \quad$ Comprimento característico dado pelo comprimento do domínio

$U_{c} \quad$ Velocidade característica

$t_{c} \quad$ Tempo característico

$\mu_{c} \quad$ Viscosidade característica

$G \quad$ Estresse isotrópico induzido pelo campo polimérico na ausência de fluido

$W_{i} \quad$ Número de Weissenberg

Re Número de Reynolds 
l Parâmetro relacionado com a extensibilidade média máxima das cadeias poliméricas da ordem do domínio

$l_{\text {top }} \quad$ Nível mais fino 


\section{Lista de Figuras}

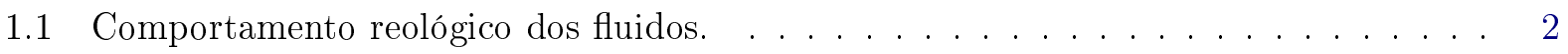

1.2 Bolhas em fluidos newtoniano e não newtoniano. . . . . . . . . . . . . . . . . 3

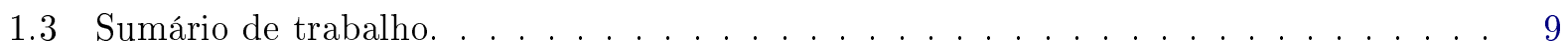

2.1 Definição de interface, fase contínua e fase dispersa. . . . . . . . . . . . . . . . . . . 12

2.2 Função indicadora e de Heaviside contínuas. . . . . . . . . . . . . . . . . . . . . 13

2.3 Representação de pontos no domínio euleriano e lagrangiano. . . . . . . . . . . . . 15

2.4 Definição do tensor viscoelástico bifásico. . . . . . . . . . . . . . . . . . . . 16

3.1 Localização das variáveis em uma célula computacional $(i, j)$ de $\mathcal{G}_{\Omega} \ldots \ldots$. . . . . . 24

3.2 Malhas euleriana e lagrangiana. . . . . . . . . . . . . . . . 26

3.3 Construção da função indicadora discreta. . . . . . . . . . . . . . . . . . 28

3.4 Função indicadora e de Heaviside discretas. . . . . . . . . . . . . . . . . . 28

3.5 Distribuição de células em uma malha uniforme em uma direção. . . . . . . . . . . . . 38

4.1 Malha composta e sua representação como união de retalhos. . . . . . . . . . . . . 52

4.2 Exemplos de malhas propriamente e não propriamente aninhadas. . . . . . . . . . . 54

4.3 Aproximação para células fantasmas em um retalho de AMR. . . . . . . . . . . . . 55

4.4 Células visíveis e recobertas na malha composta. . . . . . . . . . . . . . . . . . . . . . . . . . 56

4.5 Interpolação cúbica para célula recoberta. . . . . . . . . . . . . . . . . . . . 57

4.6 Interpolação fina-grossa. . . . . . . . . . . . . . . . . . 58

4.7 Representação de um nó da lista ligada matricial. . . . . . . . . . . . . . . . . . . . 62

4.8 Enumeração de retalhos. . . . . . . . . . . . . . . . . . . . . . . 64

4.9 Mapa de visibilidade nível base no centro da célula. . . . . . . . . . . . . . . . 65

4.10 Mapa de visibilidade nível base $+1=L_{2}$ no centro da célula. . . . . . . . . . . 66

4.11 Nível base $+2=L_{3}$ no centro da célula. . . . . . . . . . . . . . . . . . 66

4.12 Enumeração no centro da célula. . . . . . . . . . . . . . . . . . . . . . . . . . . . 67

4.13 Lista ligada matricial . . . . . . . . . . . . . . . . . . . . . . 68

4.14 Estêncil para interpolação cúbica . . . . . . . . . . . . . . . . . . . . . . . . . . . . .

4.15 Interpolação malha fina-grossa na célula $(i+1, j) \ldots \ldots \ldots \ldots$. . . . . . 70

4.16 Estêncil da interpolação fina grossa em T-Junctions. . . . . . . . . . . . . 70

5.1 Malha composta com três níveis de refinamento e sua divisão em retalhos. . . . . . . 80

5.2 Malhas e retalhos usados no cálculo de células fantasmas para Neumann. . . . . . . . 82

5.3 Malhas e retalhos usados no cálculo de células fantasmas periódicas. . . . . . . . . 83

5.4 Padrão de esparsidade da equação elíptica. . . . . . . . . . . . . . . . . . . . . 88 
5.5 Malha composta com dois níveis de refinamento e solução obtida com o PETSc. . . . 89

5.6 Erro na resolução da equação de Poisson com dois níveis de refinamento. . . . . . . . 91

5.7 Malha composta com sete níveis de refinamento. . . . . . . . . . . . . . . . 91

5.8 Interface fina-grossa em malha composta. . . . . . . . . . . . . . . . . 93

$5.9 \omega$ variável. (a) Altura e (b) zona de transição. . . . . . . . . . . . . . . . . . . 95

5.10 Malhas uniforme e composta. . . . . . . . . . . . . . . . . . . 95

5.11 Malha composta $128 \times 128 L 2=G 7 L 3$ e $\omega(x, y)$ para $h=0.4 \ldots \ldots$. . . . . . . 97

5.12 Comportamento do resíduo por iteração com os métodos BCGS e o multinívelmultigrid para $h=0.1$ (esquerda) e para $h=0.4$ (direita) . . . . . . . . . 100

5.13 Padrão de esparsidade para $h=0$ correspondente à discretização na malha composta da Figura 5.10. . . . . . . . . . . . . . . . . . . . . . . 102

5.14 C-grid e aproximação de $u$ e $v$, com o laplaciano definido nas arestas e condições Dirichlet. . . . . . . . . . . . . . . . . . . . . 104

5.15 Malha composta (à esquerda) e malha composta refinada (à direita) com a aproxi-

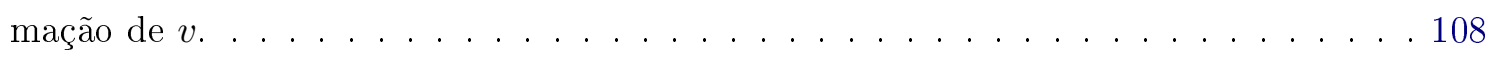

5.16 Padrão de esparsidade da matriz na equação de difusão com o método de Euler Implícito. . . . . . . . . . . . . . . . . . . . . 109

5.17 Malha composta com variação para $p$ e aproximação para $\phi$ usando ENO3 com condições de contorno mistas de Dirichlet e periódica. . . . . . . . . . . . . . 112

5.18 Malha composta empregada na verificação da equação de Stokes para um escoamento incompressível estacionário. . . . . . . . . . . . . . . . . 118

5.19 Aproximações obtidas com a solução da equação de Stokes para um escoamento incompressível estacionário de um fluido bifásico a baixo cisalhamento. Da esquerda à direta, temos a força $f_{x}$ dada pela interface na aresta vertical, a função indicadora, a pressão e a malha composta em cada caso. . . . . . . . . . . . . . . . . . . 120

6.1 Cinza: trabalho futuro a curto prazo. Branco: trabalho futuro a longo prazo. . . . . 125

B.1 Mapa de visibilidade nível base na aresta $x$ para condição de contorno de Dirichlet ou de Neumann. . . . . . . . . . . . . . . . . . . . . . . . . . . . . 132

B.2 Mapa de visibilidade na aresta $x$ para condição de contorno de Dirichlet ou de Neumann. (a) Nível base $+1=L_{2}$. (b) Nível base $+2=L_{3} . \quad \ldots \ldots \ldots$. . . . . . . 133

B.3 Mapa de visibilidade nível base na aresta $y$ para condição de contorno de Dirichlet ou de Neumann. . . . . . . . . . . . . . . . . . . . . . . . . . . . . 134

B.4 Mapa de visibilidade na aresta $x$ para condição de contorno de Dirichlet ou de Neumann. (a) Nível base $+1=L_{2}$. (b) Nível base $+2=L_{3} . \ldots \ldots \ldots$. . . . . . 135

B.5 Malha composta para caso periódico. . . . . . . . . . . . . 135

B.6 Mapa de visibilidade na aresta $x$ caso periódico . . . . . . . . . . . 136

C.1 Enumeração na aresta $x$ para condições de contorno de Dirichlet ou de Neumann. . . 138

C.2 Enumeração na aresta $y$ para condições de contorno de Dirichlet ou de Neumann. . . 138

C.3 Enumeração na aresta $x$ para o caso periódico. . . . . . . . . . . . . . . . 139 


\section{Lista de Tabelas}

5.1 Erro e ordem de convergência no cálculo dos valores das células fantasmas empregando o produto matriz-vetor e o cálculo usual nível-a-nível, retalho-a-retalho. Condição de contorno de Dirichlet. . . . . . . . . . . . . . . . . . 80

5.2 Erro e ordem de convergência no cálculo dos valores das células fantasmas empregando o produto matriz-vetor e o cálculo usual nível-a-nível, retalho-a-retalho. Condição de contorno de Neumann. . . . . . . . . . . . . . . . . . . . . . . . 80

5.3 Erro e ordem de convergência no cálculo dos valores das células fantasmas empregando o produto matriz-vetor e o cálculo usual nível-a-nível, retalho-a-retalho. Condição de contorno periódica. . . . . . . . . . . . . . . . . . . 80

5.4 Tempo no cálculo de valores em células fantasmas. Produto matriz-vetor vs. cálculo convencional. . . . . . . . . . . . . . . . . . . . . . . . 81

5.5 Informação sobre a quantidade de retalhos por nível da Figura $5.2 \ldots$. . . . . . . . 84

5.6 Tempo no cálculo de valores em células fantasma. Produto matriz-vetor vs. cálculo

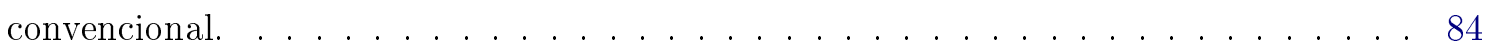

5.7 Informação sobre a quantidade de retalhos por nível da Figura 5.3. . . . . . . . . . . 84

5.8 Erro, ordem de convergência e número de iterações para a equação de Poisson em malha uniforme. Variáveis no centro de célula. . . . . . . . . . . . . . . . 89

5.9 Erro, ordem de convergência e número de iterações para a equação de Poisson em malha composta com dois níveis. Variáveis no centro de células. . . . . . . . . . . . 90

5.10 Informação da matriz para malha uniforme e composta com 2 níveis de refinamento da Figura 5.5. . . . . . . . . . . . . . . . . . . . 90

5.11 Informação sobre a quantidade de células da malha composta com sete níveis de refinamento. . . . . . . . . . . . . . . . . . . . . . 9 90

5.12 Erro e número de iterações em malha composta com sete níveis. . . . . . . . . . . . 92

5.13 Erro, ordem de convergência e número de iterações com $h=10^{3}, d=2$ e dois níveis

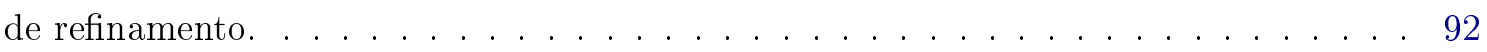

5.14 Erro e ordem de convergência com $h=0$ em malha uniforme (à esquerda) e em malha composta (à direita), usando condição de contorno periódica em todas as direções. 96

5.15 Erro e ordem de convergência com $h=0$ em malha composta. . . . . . . . . . . . 97

5.16 Comparação entre métodos do PETCs e o método multinível-multigrid "nativo" do AMR2D $(h=0$ em malha composta). . . . . . . . . . . . . . . . 98

5.17 Comparação de pré-condicionadores do PETCs fixando o solver BCGS ( $h=0 \mathrm{em}$

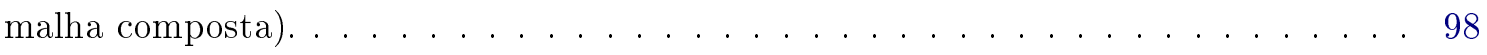


5.18 Comparação em paralelo entre os métodos GMRES e BCGS do PETSc e o método multinível-multigrid "nativo" do AMR2D. . . . . . . . . . . . . . . . . . . . . . . . . 99

5.19 Comparações de paralelismo $(h=0.1$ em malha composta $) . \quad$. . . . . . . . . . . . . 99

5.20 Comparações de paralelismo $(h=0.4$ em malha composta $) .. . . . . .100$

5.21 Diferentes valores de $h$ para o BCGS em malha composta. . . . . . . . . . . . . . . 100

5.22 Solução manufaturada em malha composta com dois níveis de refinamento e $h=0.0$. 101

5.23 Diferentes valores de $h$ em malha composta. . . . . . . . . . . . . . . . . . . . 101

5.24 Informações da matriz para $\omega=1 / \rho$ e diferentes valores de $h$. . . . . . . . . . . 102

5.25 Erro e razão de convergência para $u$ e $v$ para a solução da equação de Poisson, com condições de contorno de Dirichlet. . . . . . . . . . . . . . . . . . . . . . . . . . . . . 104

5.26 Erros, ordem de convergência e número de iterações na equação de difusão com o método de Euler Implícito. . . . . . . . . . . . . . . . . . . . . . . . . . . . 109

5.27 Erro, ordem de convergência e número de iterações na equação de difusão com o Método de Gear. . . . . . . . . . . . . . . . . . . . . . . . . . . . . . . . 110

5.28 Erro e ordem de convergência usando ENO3 com condição de Dirichlet para malhas uniforme e composta. . . . . . . . . . . . . . . . . . . . . . . . . 111

5.29 Erro e ordem de convergência usando ENO3 com condição mista de Dirichlet e periódica. Malha composta. . . . . . . . . . . . . . . . . . . . . . . . . . 112

5.30 Erro e ordem de convergência em malha uniforme e em malha composta empregando ENO3 com condição de contorno mista de Dirichlet e periódica. . . . . . . . . . . . . 113

5.31 Erro e ordem de convergência nas malhas uniforme (L1) e composta (L3) para cada uma das componentes do tensor $\mathbf{S}$ com o Modelo Oldroyd-B. . . . . . . . . . . . . . 114

5.32 Erro e ordem de convergência para as componentes do tensor viscoelástico com Mod-

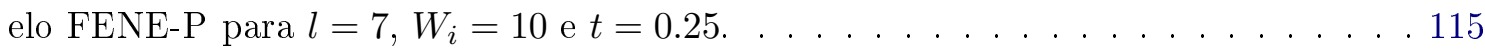

5.33 Erro e ordem para cada direção da velocidade e a pressão usando o IMEX com parâmetros $\left(\frac{1}{8}, \frac{1}{2}\right)$ e $\left(0, \frac{1}{2}\right)$, na solução das equações de Navier-Stokes. . . . . . . . . . 116

5.34 Erro e ordem de convergência na solução das equações de Navier-Stokes viscoelásticas. 117

5.35 Erro e ordem de convergência na solução das equações de Stokes para um escoamento incompressível estacionário monofásico. . . . . . . . . . . . . . . . . . . . . . . . 118

5.36 Número de iterações do Método BiCG-Stab para cada malha composta empregada e norma do divergente da velocidade.

5.37 Erro e razão de convergência com a solução manufatura da equação de Stokes com o termo viscoelástico. . . . . . . . . . . . . . . . . . . . . . . . 121 


\section{Lista de Algoritmos}

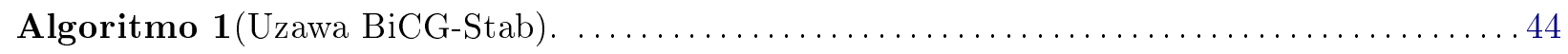

Algoritmo 2(SAMR Método de Uzawa com BiCG-Stab retalho-a-retalho) . . . . . . . . . . . 73 


\section{Capítulo 1}

\section{Introdução}

\subsection{Motivação científica}

Fluidos bifásicos viscoelásticos têm um papel muito importante em aplicações industriais e constantemente aparecem na Medicina, na Biologia, na Química, na indústria farmacêutica, na engenharia de petróleo, entre outras, dado que estes fluidos estão presentes em emulsões, na dinâmica de microestruturas, na produção de tintas, na mistura de polímeros, na extração de petróleo, em processos alimentícios, etc. As propriedades reológicas de escoamentos bifásicos nos quais uma das fases não é newtoniana (fluidos complexos) são bem diferentes das propriedades de qualquer fluido newtoniano. A importância destes escoamentos, numa vasta gama de aplicações médicas, industriais e da engenharia, destaca a necessidade de entendermos melhor esta classe de fluidos.

Nas décadas de 50 e de 60 , o desenvolvimento comercial de monômeros para a produção de novos polímeros cresceu bastante pois, neste período, iniciou-se o emprego de processos industriais nos quais dois ou mais polímeros eram misturados para criar um novo material com diferentes propriedades físicas. Por serem mais econômicos, o desenvolvimento de novas técnicas para a modificação dos polímeros já existentes, passou a ser muito atraente e, hoje em dia, ocupa um espaço importante na área de pesquisa de dinâmica dos fluidos.

Pelo comportamento dos fluidos, estes podem ser classificados em dilatantes, nos quais a resistência aumenta com o aumento da tensão aplicada (e.g. areia movediça ou suspensões com amido), pseudoplásticos, nos quais a resistência diminui com o aumento da pressão aplicada (e.g. tintas e sangue) e plástico de Bingham, os quais precisam de uma tensão finita para começar a escoar (e.g. pasta de dente e ketchup). A Figura 1.1 mostra o comportamento destes fluidos não newtonianos e dos fluidos newtonianos, quando se é aplicada uma tensão neles.

Dos fluidos não newtonianos, as emulsões são o ponto principal na produção de materiais avançados, particularmente quando uma ou ambas fases são não newtonianas. Por exemplo, há um grande interesse na mistura de polímeros incompatíveis devido ao atrativo de suas propriedades mecânicas e pela necessidade de reciclagem de materiais plásticos. O processamento destes materiais exige um detalhado conhecimento reológico no derretimento de emulsões e isto, por sua vez, requer uma melhor compreensão de interações entre gotículas num escoamento bifásico.

Assim sendo, a investigação da dinâmica de interfaces de separação entre dois fluidos, tais como as que delimitam gotas e bolhas em escoamentos bifásicos com um componente viscoelástico são um problema de relevante interesse tecnológico. Esta é precisamente a grande motivação que impulsiona nosso trabalho: o estudo, o desenvolvimento e o teste de metodologias numéricas robustas e eficientes 


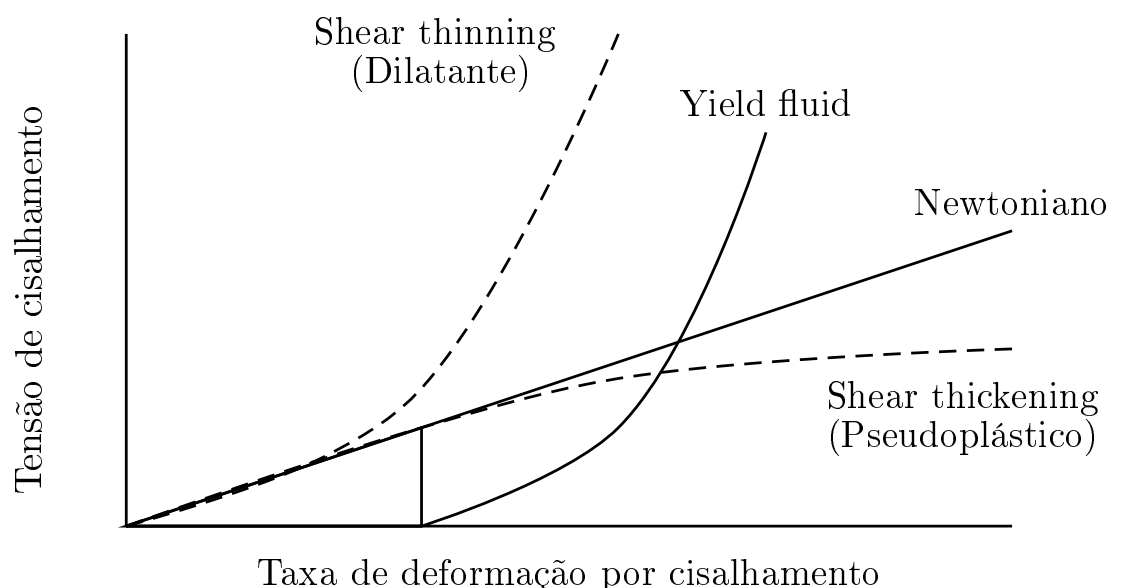

Figura 1.1: Comportamento reológico dos fluidos.

que, quando combinadas, permitam, em última análise, propor técnicas numéricas para simular escoamentos incompressíveis de fluidos bifásicos nos quais se possa ter uma fase viscoelástica.

\subsection{Metodologia e revisão da literatura}

Nosso trabalho é orientado ao desenvolvimento de técnicas e metodologias numéricas as quais, quando combinadas, possibilitem a simulação computacional eficiente e robusta do escoamento incompressível de fluidos bifásicos nos quais ao menos uma das fases possa ser viscoelástica (não newtoniana). Na literatura, existem várias equações constitutivas que modelam os diferentes tipos de fluidos viscoelásticos. Estas equações procuram modelar a presença de cadeias poliméricas, interconectadas e dispersas no fluido, sendo tais cadeias responsáveis pelo efeito de "memória" nos fluidos viscoelásticos.

Um modelo que descreve uma relação entre a velocidade do fluido, as propriedades poliméricas e o tensor de tensões poliméricas é o Modelo Oldroyd-B [99, 100]. Este é um dos modelos constitutivos mais simples que há para a modelagem matemática de fluidos viscoelásticos [27, 44, 99]. O Modelo Oldroyd-B pode ser deduzido de princípios microscópicos e da aplicação da Lei de Hooke, assumindo que as cadeias poliméricas comportam-se como molas que podem ser estendidas infinitamente. Este modelo é criticado dado que fisicamente a extensão das cadeias poliméricas é finita.

O Modelo FENE (Finite Extendible Nonlinear Elastic) baseia-se na teoria cinética de moléculas poliméricas com extensão elástica finita e não linear. Desafortunadamente, para determinar o tensor viscoelástico com este modelo é necessário ter informação estatística da configuração molecular o qual aumenta a dimensão do sistema [60].

O Modelo FENE-P, uma variação do Modelo FENE proposta por Peterlin [19], modela fluidos viscoelásticos empregando uma aproximação estatística para ter um sistema fechado de equações no espaço físico e assim conseguir uma solução numérica. Este modelo tem um parâmetro que controla a extensibilidade média máxima das cadeias poliméricas. Se a extensão máxima é muito grande, este modelo se comporta como o Modelo Oldroyd-B.

Recentemente surgiram outros modelos para estudar propriedades de fluidos viscoelásticos como, por exemplo, a técnica log-conformation [43].

As equações diferenciais para estes modelos são equações hiperbólicas e há que se ter muito 
cuidado na solução numérica das mesmas. O uso de esquemas como o ENO (Essential NonOscillatory) [51, 93] de alta ordem é crucial na discretização do termo advectivo nos modelos constitutivos (e.g. Oldroyd-B e FENE-P) usados na modelagem de fluidos viscoelásticos. Este esquema diminui a dissipação numérica nos problemas hiperbólicos, tendo um tratamento especial nas descontinuidades. Discretizações temporais de alta ordem são necessárias, diminuindo as restrições numéricas como as que aparecem ao se discretizar o tensor de tensões poliméricas com o Método das Diferenças Finitas e o Método de Euler explícito [43, 54]. Shu e Osher [93] propuseram para a discretização temporal de equações hiperbólicas métodos de Runge-Kutta TVD (total variation diminishing).

No caso de simulações de bolhas, a solução numérica de modelos matemáticos demanda grande esforço computacional. Este esforço aumenta quando é necessária alta precisão e rapidez computacional. Assim, na captura de detalhes e de fenômenos locais, sabendo que a região de interesse pode ser muito pequena quando comparada com o domínio computacional, uma discretização usando malhas uniformes é bastante ineficiente. Desta forma, o uso de malhas bloco-estruturadas com refinamento adaptativo localizado [16], técnica conhecida como SAMR (Structured Adaptive Mesh Refinement), é uma solução eficiente e ótima na busca de precisão.

As malhas empregadas em nosso trabalho, conhecidas como "malhas compostas" ou "malhas bloco-estruturadas" (SAMR - Structured AMR), são aquelas introduzidas por Berger e Oliger [16, $17,18]$, formadas por blocos retangulares ("retalhos") de refinamentos que se adaptam e recobrem regiões específicas do domínio.

Seguindo a técnica proposta por Berger e Colella [16, 17, 18], Villar implementou um código computacional para problemas bidimensionais ao qual nós nos referiremos em nosso trabalho como "AMR2D" [32, 111]. Em seu trabalho de doutorado, Villar [111] descreve a estrutura de dados necessária à implementação da técnica SAMR. Nossos desenvolvimentos têm por base aquela implementação a qual, ao longo do tempo, foi sendo aprimorada e estendida de diversas maneiras que ficarão claras ao longo dos vários capítulos a seguir.

Fluidos viscoelásticos, na verdade os não newtonianos em geral, têm propriedades materiais e um comportamento diferente dos fluidos newtonianos. As diferenças têm origem nas propriedades microscópicas, na estrutura molecular de tais fluidos. É interessante, pela grande quantidade de aplicações na prática, estudar os fenômenos físicos exibidos por fluidos viscoelásticos. Exemplos marcantes são os de sua rigidez e dos efeitos inerciais, introduzidos pelas não-linearidades e pela elasticidade [60, 75]. Na Figura 1.2, mostramos a diferença da geometria adquirida por uma bolha em meios newtoniano e não newtoniano, respectivamente.

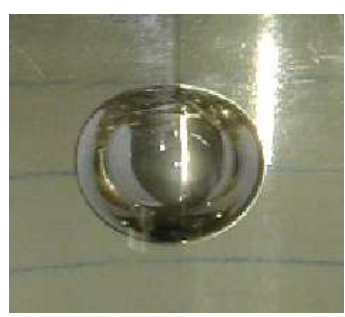

(a)

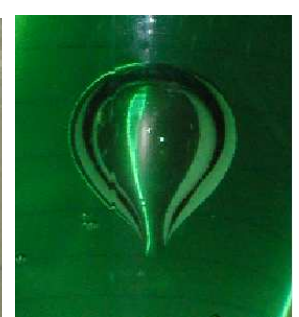

(b)

Figura 1.2: Bolhas em fluidos (a) newtoniano e (b) não newtoniano. E. J. Hinch [52].

As equações que modelam matematicamente a dinâmica de estruturas imersas em fluidos newtonianos e não newtonianos são as equações de Navier-Stokes em conjunto com a força da interface 
imersa que interage com o fluido e o tensor viscoelástico. Pela variabilidade de escalas de comprimento entre as aplicações, que poderiam ser macro ou até nano dependendo do caso, é conveniente trabalharmos com a forma adimensional dessas equações. A adimensionalização permite fazermos testes de interesse de acordo com as propriedades dinâmicas de escoamentos como, por exemplo, no caso de movimento de estruturas imersas em fluidos com baixo número de Reynolds [44, 99].

Ao modelarmos fluidos bifásicos, é preciso determinar de forma correta e eficiente cada uma das propriedades físicas das diferentes fases envolvidas no escoamento. Técnicas como as de "acompanhamento de interface" (front-tracking methods [31, 78, 81, 106, 107]) identificam as propriedades das fases imiscíveis por intermédio do uso de uma função indicadora. Existem diferentes maneiras de obtermos a função indicadora. Ela pode ser obtida empregando-se algoritmos de Geometria Computacional [31] ou resolvendo-se equações a derivadas parciais do tipo elíptico [92, 107] ou hiperbólico [73, 94]. No caso de "técnicas de captura" (front-capturing methods [73, 74, 92]), tais fases são naturalmente identificadas por uma função escalar, como no caso de métodos level set [73, 74, 92]. Entretanto, em tais métodos, cálculos de propriedades geométricas como o da normal e da curvatura da interface de separação entre as fases são mais difíceis de serem feitos de maneira precisa. Ao combinarmos qualquer dessas técnicas com refinamento adaptativo de malhas, como os oriundos da técnica SAMR, é possível realizarmos simulações computacionais da dinâmica de fluidos complexos contendo estruturas imersas que variam no tempo. No presente trabalho, o controle das propriedades e o modelo válido em cada material ou fluido é feito com o uso de uma função indicadora eficiente proposta por Ceniceros e Roma [31, 111] e usamos uma malha lagrangiana separada da malha euleriana para seguir explicitamente o movimento da interface (no estilo do Método da Fronteira Imersa [32, 81, 107]).

Em aplicações nas quais o número de Reynolds é "muito pequeno", podemos obter a partir da adimensionalização das equações de Navier-Stokes (Apêndice A) a equação de Stokes para escoamentos estacionários. No contexto de "baixo Reynolds", temos aplicações como a natação de microorganismos [98] e o escoamento de polímeros viscosos [57, 99, 100, 101], entre outras. Escoamentos a baixo número de Reynolds ocorrem quando a velocidade do fluido é baixa ou a viscosidade é muito alta ou as escalas de comprimento do fluido são muito pequenas quando comparadas com um comprimento característico. Claro, os adjetivos "alto", "baixo", "pequeno" e "grande" dependem de comparações com escalas características de velocidade, viscosidade, comprimento, etc., que caracterizam a dinâmica e as propriedades físicas nas aplicações de interesse. É de nosso interesse estudar a dinâmica de escoamentos a baixo número de Reynolds no caso de fluidos viscoelásticos. Desse interesse decorre a necessidade de estudarmos a resolução da equação de Stokes.

Para resolver a equação de Stokes no caso estacionário, partimos do estudo de um fluido newtoniano com baixo número de Reynolds, escoando de maneira incompressível. Tal escoamento pode ser modelado matematicamente pelas equações

$$
\begin{aligned}
-\nabla p+\nabla^{2} \mathbf{u} & =\mathbf{f}, \\
\nabla \cdot \mathbf{u} & =0,
\end{aligned}
$$

onde $p$ é a pressão, u é o campo de velocidade e f é o termo forçante.

Procuramos, em um primeiro momento, trabalhar com as equações de continuidade e de quantidade de movimento de maneira acoplada, olhando para o problema discreto como sendo dado por 
um único e grande sistema linear, cujas incógnitas eram as componentes da velocidade e a pressão. Como é natural dentro do contexto de malhas bloco-estruturadas, procuramos resolver esse sistema linear usando um método multinível-multigrid. Tais métodos têm sido implementados e reportados no contexto SAMR por vários grupos de pesquisa [2, 3, 29, 32], em várias aplicações.

No caso de escoamentos estacionários modelados pelas equações de Stokes, entretanto, identificamos logo de partida um problema: ao ter as equações acopladas em um único sistema, obtemos um bloco de zeros na diagonal do sistema linear e, portanto, obtemos um sistema do tipo saddle point [21, 22, 36, 117], para o qual a relaxação de Gauss-Seidel, frequentemente usada em métodos multigrid, não pode mais ser aplicada $[72,114]$. Buscamos alternativas para solucionar esse problema: a necessidade de um outro tipo de relaxador. Segundo a literatura, poderíamos empregar relaxadores que usam uma "transformação de iteração" como, por exemplo, a relaxação distribuída e o precondicionamento [8,70,115], ou ainda relaxadores que acrescentam uma "pressão artificial", como o Método SIMPLE (Semi-Implicit Method for Pressure Linked) [6, 77] e o Método SIMPLER (a SIMPLE Revised algorithm) [77]. Implementamos uma relaxação baseada nos métodos que acrescentam a pressão artificial. Desafortunadamente, em malhas bloco-estruturadas, não obtivemos sucesso. Acreditamos que o motivo seja a implementação das condições de contorno e a condição de compatibilidade usada para a pressão no contexto SAMR [49, 64]. Mais investigações seriam necessárias para esclarecer esse ponto.

Em uma outra abordagem, decidimos resolver o sistema linear desacoplando a velocidade da pressão empregando o Método de Uzawa [4, 7, 56, 57]. A implementação do Método de Uzawa [4] empregando malhas compostas bloco-estruturadas SAMR no contexto do Método das Diferenças Finitas está dentre as contribuições expressivas deste trabalho. O uso daquele método em malhas adaptativas, do nosso conhecimento, é encontrado na literatura empregando-se apenas o Método dos Elementos Finitos [40, 56, 59]. Como nós usamos o Método das Diferenças Finitas e o SAMR, conseguimos discretizar de maneira simples e ao mesmo tempo adaptativa o método, comparativamente à discretização via o Método dos Elementos Finitos.

O Método de Uzawa desacopla a pressão da velocidade, formando dois sistemas lineares que podem ser resolvidos de forma iterativa. Na literatura, o principal método usado para a solução destes sistemas é o Método dos Gradientes Conjugados (CG [56, 59, 69]). Entretanto, encontramos também implementações empregando os métodos SOR (Successive Over-Relaxation $[13,117,118]$ ), GMRES (Generalized Minimal RESidual method [13, 22]) e o BiCG-Stab (BiConjugate Gradient Stabilized method $[57,108])$, dentre outros.

Com o Método de Uzawa, o sistema linear que obtemos para a pressão usando malhas adaptativas SAMR não é simétrico. Assim, usamos o Método BiCG-Stab [13, 57, 108], o qual permite encontrar soluções para sistemas não simétricos. Como resultado, ao desacoplar a pressão da velocidade com o Método de Uzawa em conjunto com o Método BiCG-Stab, precisamos resolver duas equações elípticas em cada iteração do Método BiCG-Stab. Estas equações são para variáveis definidas no meio das arestas das células computacionais e para sua solução o primeiro esquema que empregamos foi o Método de Gauss-Seidel preto-vermelho [23, 104] (red-black) com a técnica SAMR, inicialmente sem sucesso no contexto do Método BiCG-Stab.

A busca pelas razões que levavam à dificuldade de convergência do Método BiCG-Stab em malhas compostas bloco-estruturadas fez com que estudássemos de maneira mais profunda a determinação de valores fantasmas (detalhes mais adiante) e, em particular, as interpolações responsáveis 
por sua determinação, as quais estão intimamente ligadas à troca de informação entre os diversos "retalhos" de malhas entre os diferentes níveis de refinamento. Essa busca levou de forma natural ao interesse por uma representação matricial explícita das interpolações para os valores fantasmas e da forma discreta das equações diferenciais envolvidas em nosso trabalho. Além do entendimento mais aprofundado da formação de valores fantasmas que tais representações matriciais dos sistemas lineares envolvidos permitiria, vislumbramos a possibilidade do uso de bibliotecas numéricas existentes e o acesso que elas dariam a vários métodos numéricos e seus pré-condicionadores, dedicados à resolução de sistemas lineares - inclusive suas versões paralelas. A formulação matricial explícita de nossos problemas em SAMR abriria um mundo totalmente novo de possibilidades a serem exploradas.

São raríssimos os relatos sobre a formação e sobre as propriedades dessas matrizes no contexto SAMR [41, 42, 53, 83]. Tal abordagem ainda foi pouquíssimo explorada desde as primeiras propostas envolvendo este tipo de malha espacial. Consideramos que estejam dentre nossas principais contribuições o desenvolvimento de algoritmos, sua implementação e testes no contexto de valores fantasmas em malhas SAMR e na discretização de algumas equações diferenciais parciais, fundamentais à resolução dos modelos matemáticos apresentados. A formação das representações matriciais explícitas é bastante complexa e o nível de habilidade exigido em sua obtenção elevado. Entretanto, elas são extremamente úteis no estudo das propriedades dos sistemas lineares formados em malhas bloco-estruturadas (e.g. definição e simetria, número de condicionamento, raio espectral e autovalores, dentre outras) e por permitem o uso direto de bibliotecas numéricas externas de Álgebra Linear Computacional (métodos, pré-condicionadores e paralelismo). Muito pouco se sabe com certeza sobre os sistemas lineares em malha composta. Pela oportunidade de esclarecermos e oferecermos informação nesse sentido, tal abordagem ocupa o centro de nossa atenção e esforço e dedicamos grande parte do capítulo de resultados a ela, sendo ela o "núcleo" de nosso trabalho.

A representação matricial explícita para uma equação de Poisson assume posição de destaque em nosso trabalho: primeiro, porque equações elípticas precisam ser resolvidas nas iterações do Método de Uzawa com o BiCG-Stab nas equações de Stokes (escoamentos incompressíveis estacionários). Segundo, porque ao resolver as equações de Navier-Stokes para escoamentos bifásicos com o do Método da Projeção de Chorin-Temam [34, 97] também passamos pela resolução de uma equação elíptica para a correção da pressão. Em malha composta e com a metodologia multinívelmultigrid [65, 85, 102], quando há um "salto grande" entre as massas específicas das fases envolvidas (e.g. ar e água), não é incomum observarmos problemas de convergências (e.g. elevados números de V-ciclos devem ser empregados ou mesmo a estagnação da queda do resíduo) [111]. Nesse último caso, ao empregarmos a representação matricial explícita para a discretização da equação de Poisson para a correção da pressão, conseguiremos comparar diferentes métodos de resolução e obter a convergência em casos nos quais o método multinível-multigrid falha.

Durante o desenvolvimento de nosso trabalho, realizamos uma viagem com duração de um mês à University of California at Santa Barbara para desenvolver pesquisa com nosso co-orientador, Professor Dr. Hector D. Ceniceros do Departamento de Matemática. Durante essa estadia foi implementado o esquema ENO de terceira ordem para a solução de equações diferenciais hiperbólicas [51, 73, 93] em malha composta e foram observados quais aplicações seriam possíveis com essa ferramenta que estava implementada. Esse nosso interesse e desenvolvimentos de representações matriciais explícitas para valores fantasmas e discretizações em malhas compostas, permitiu nossa 
participação em um projeto conjunto FEMEC-UFU, ICMC-USP e IME-USP com financiamento Petrobras. Atualmente, os algoritmos e as estruturas de dados propostas em nosso trabalho estão sendo estendidas para versões tridimensionais.

\subsection{Objetivos}

De maneira geral, nosso objetivo é o de pavimentar de maneira firme o caminho que levará a metodologias numéricas robustas capazes de lidarem de maneira eficiente com aplicações complexas envolvendo fluidos bifásicos com fases imiscíveis, nos quais podemos ter ao menos uma das fases dadas por um fluido viscoelástico. Esta pavimentação é feita por intermédio do estudo de alguns problemas-modelo essenciais, dados por equações diferenciais parciais. No contexto de nossa motivação científica, fluidos newtonianos e não newtonianos em escoamentos incompressíveis transientes e estacionários, tais problemas-modelo são dados por equações elípticas e parabólicas, com a função incógnita localizada em posições diferentes das células computacionais que formam a discretização no espaço do domínio computacional (e.g. centros de células e meios de arestas). Nosso ponto de partida e ferramenta de trabalho principal é o código AMR2D, desenvolvido originalmente por Villar [111] para simular computacionalmente escoamentos incompressíveis transientes de fluidos newtonianos bifásicos. Tal código emprega o Método da Projeção de Chorin-Temam em consonância com métodos implícitos-explícitos, malhas bloco-estruturadas SAMR e o Método da Fronteira Imersa [32]. Ao propormos os algoritmos principais para a formação das matrizes explícitas, tomamos especial cuidado para que as implementações que fizemos em duas dimensões pudessem ser estendidas sem grande dificuldade e de maneira natural aos códigos já existentes tridimensionais (AMR3D [71, 90]).

Nossos objetivos específicos são:

1. Rever o algoritmo tradicional para o cálculo de valores fantasmas, baseado em operações retalho-a-retalho e laços de varredura nível-a-nível e implementado no AMR2D, com o intuito de alterá-lo de maneira a permitir a implementação de outros métodos numéricos para resolver sistemas lineares, diferentes dos métodos multinível-multigrid;

2. Obter representações matriciais explícitas, inclusive propor estruturas de dados apropriadas, para o cálculo de valores fantasmas e para discretizar em malha composta os problemas-modelo essenciais mencionados acima;

3. Implementar na malha composta o Método BiCG-Stab com algoritmo baseado em operações retalho-a-retalho e laços de varredura nível-a-nível, necessário para resolver as equações de Stokes estacionárias com o Método de Uzawa;

4. Aproximar em malha composta a solução da equação hiperbólica para as equações constitutivas do tensor viscoelástico (modelos Oldroyd-B e FENE-P).

Aproveitando o momento, tomamos como objetivo de nosso trabalho, mas de maneira apenas secundária, a conjunção dos conhecimentos adquiridos no processo acima para dar os passos iniciais na direção do desenvolvimento das metodologias adaptativas que, em trabalhos futuros, culminarão na simulação computacional efetiva dos escoamentos envolvidos nas aplicações mencionadas. 
Representamos nossos objetivos na forma de um fluxograma, mostrado na Figura 1.3. Os blocos (a) e (h) na cor azul contêm contribuições dadas por nosso trabalho na implementação AMR2D: em (a), ao depurar e solucionar detalhes na implementação da subrotina para o cálculo de valores fantasmas (Seção 4.1.2) e, em (b), as contribuições ao incluir a forma paramétrica do Método IMEX e o tensor viscoelástico na implementação existente para solucionar as equações de Navier-Stokes em escoamentos incompressíveis (seções 3.2.2 e 3.4). Nossas contribuições mais salientes estão na cor preta na Figura 1.3. Em amarelo, estão os itens nos quais a nossa pesquisa dá passos iniciais e olha para desenvolvimentos em trabalhos futuros. Em (e), temos em mente a aplicação de um escoamento cisalhante de uma bolha circular no contexto dado pelas equações de Stokes, na qual observamos a deformação geométrica da forma esperada mas, para qual, precisaremos ainda estudar com mais profundidade a física envolvida no problema (Subseção 5.6.2). Na implementação do modelo bifásico para fluidos viscoelásticos foram iniciados os primeiros passos e sua continuidade é trabalho futuro, e estão representados na cor cinza pelos blocos (i) e (j).

\subsection{Organização do presente trabalho}

Os desenvolvimentos apresentados poderão ser usados futuramente no estudo do comportamento de bolhas em fluidos newtonianos [32] e em fluidos viscoelásticos. A metodologia empregada permitirá também a simulação computacional de escoamentos bifásicos, transientes e estacionários, em domínios complexos (e.g. canais com e sem estenose [54]).

O presente trabalho está estruturado como descrito a seguir. O Capítulo 1 apresenta as motivações científicas que nortearam nossos esforços e descreve nossos objetivos, tanto de maneira genérica como específica. Com relação à revisão da literatura, optamos por distribuí-la ao longo do texto, fornecendo informações adicionais sempre que necessário sobre o contexto em foco. Os modelos matemáticos para escoamentos incompressíveis de um fluido bifásico nos regimes transiente e estacionário, nos quais uma das fases poderá ser viscoelástica, são apresentados no Capítulo 2. Também nele são apresentados os modelos matemáticos constitutivos do tensor viscoelástico (modelos Oldroyd-B e FENE-P) e o tratamento da interface de separação entre as fases via Método da Fronteira Imersa [81, 81]. O Capítulo 3 apresenta uma descrição dos métodos numéricos e da discretização das equações utilizadas na solução numérica das equações propostas no capítulo de modelos matemáticos quando a discretização espacial é dada por uma malha uniforme. Isto facilita sobremaneira a exposição e o entendimento das técnicas empregadas. No Capítulo 4, descrevemos malhas compostas bloco-estruturadas do tipo SAMR, sua geração e propriedades, além de fornecermos a descrição detalhada de como se obtêm nelas os valores fantasmas. A representação matricial que propusemos para solucionar, por meio de bibliotecas externas, sistemas lineares oriundos da discretização de equações diferenciais usando a técnica SAMR também se encontra, para variáveis definidas em centros de célula, no Capítulo 4 (detalhes para variáveis em meio de arestas podem ser encontrados nos apêndices B e C). No Capítulo 5, apresentamos as verificações numéricas relativas à abordagem matricial para os problemas-modelo essenciais mencionados anteriormente e resultados preliminares relativos à metodologia para simulação computacional dos escoamentos de interesse futuro. Predominantemente, verificamos a correta implementação dos algoritmos desenvolvidos por intermédio da comparação dos resultados numéricos com resultados analíticos empregando a estratégia de soluções manufaturadas [23, 95]. Fazemos nossas considerações finais no Capítulo 6. 


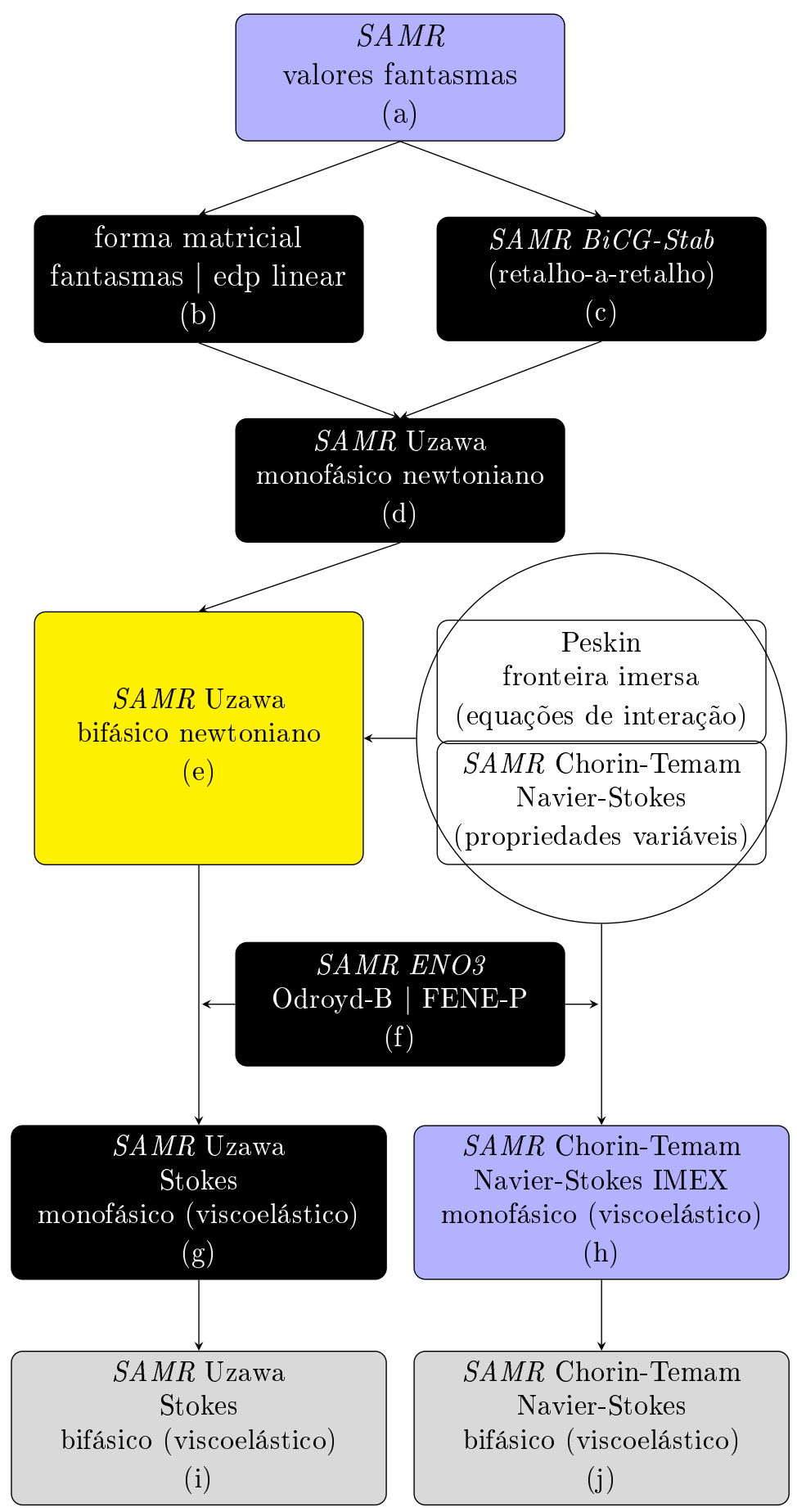

Figura 1.3: Branco: conhecimento anterior (AMR2D). Azul: contribuições. Amarelo: primeiras investigaçôes. Preto: trabalho original. Cinza: trabalho futuro. 
Finalmente, os apêndices A, B e C contêm informações que suplementam o presente trabalho. 


\section{Capítulo 2}

\section{Modelos Matemáticos}

Neste Capítulo, apresentamos os modelos matemáticos bidimensionais que descrevem a dinâmica de um escoamento incompressível de um fluido bifásico nos regimes transiente $(R e>0)$ e estacionário $(R e=0)$ nos quais uma das fases poderá ser viscoelástica (fluido não newtoniano). Dedicamos a Seção 2.1 à discussão da modelagem matemática de escoamentos transientes de fluidos bifásicos. Para discernir entre a fase dispersa e a fase contínua, empregamos uma função indicadora de fluido no contexto de um método híbrido front-tracking/front-capturing [32, 106, 107]. Por um lado, a posição dos pontos da interface de separação entre as fases é conhecida e acompanhada de maneira explícita utilizando uma metodologia lagrangiana (front-tracking) (Seção 2.2). Ao mesmo tempo, por outro lado, sua posição é empregada no cálculo de uma função que a tem como curva de nivel zero - estratégia típica de métodos de captura de interface como o Método da Curva de Nível (Level Set Method). Tal função auxilia na identificação das fases como mostraremos. Na Seção 2.3, apresentamos dois modelos para o tensor viscoelástico: o Modelo Oldroyd-B e o Modelo FENE-P. A ideia por trás destes modelos é caracterizar moléculas de polímeros com longas cadeias que exibem e agregam propriedades elásticas ao fluido. Na Seção 2.4, mostramos o modelo adimensional, ressaltando o caso em que o número de Reynolds é pequeno - situação da qual obtemos o modelo matemático para escoamentos bifásicos incompressíveis e estacionários (equação de Stokes). Finalmente, na Seção 2.5, fazemos um sumário dos modelos apresentados.

\subsection{Escoamento incompressível de um fluido bifásico}

Resolveremos as equações do escoamento num domínio retangular $\Omega$ cujas condições de contorno são uma combinação escolhida dentre as condições de Dirichlet, de Neumann e periódica. O fluido pode ser composto por até duas fases imiscíveis cujas propriedades materiais se mantêm constantes. Interfaces de separação entre fases diferentes estão sujeitas a forças de tensão superficial. Nosso ponto de partida para modelar tal escoamento é o Modelo de Um Fluido proposto por Tryggvason e colaboradores [105, 106, 107].

Neste contexto, a dinâmica do sistema bifásico é modelada matematicamente pelas equações de Navier-Stokes compostas pela equação da quantidade de movimento linear e da continuidade, 
respectivamente dadas por

$$
\begin{aligned}
\rho(\mathbf{x})\left[\frac{\partial \mathbf{u}}{\partial t}+(\mathbf{u} \cdot \nabla) \mathbf{u}\right] & =-\nabla p+\nabla \cdot\left[\mu(\mathbf{x})\left(\nabla \mathbf{u}+\nabla \mathbf{u}^{T}\right)\right]+\alpha_{p} \nabla \cdot \mathbf{S}(\mathbf{x})+\mathbf{f} \\
\frac{\partial \rho(\mathbf{x})}{\partial t}+\nabla \cdot(\rho(\mathbf{x}) \mathbf{u}) & =0
\end{aligned}
$$

onde $\mathbf{u}=(u, v)$ é o campo de velocidade, $p$ a pressão, $\rho$ é a massa específica do fluido, $\mu$ é a viscosidade dinâmica, $\mathbf{S}$ é o tensor de tensões produzido por macromoléculas, $\mathbf{f}=\left(f_{u}, f_{v}\right)$ são forças externas que atuam no fluido (e.g. da gravidade e de tensão superficial) e $\alpha_{p}$ é uma constante que define se o fluido é newtoniano ou não newtoniano.

A constante $\alpha_{p}$ só tomará os valores 0 ou 1 . Quando $\alpha_{p}$ for 1 deixamos de ter uma relação linear entre a viscosidade e a velocidade do fluido e o fluido torna-se não newtoniano. Para fixarmos as ideias, consideremos o domínio $\Omega$ (domínio euleriano) e a interface $\Gamma$ (domínio lagrangiano) representados na Figura 2.1(a).

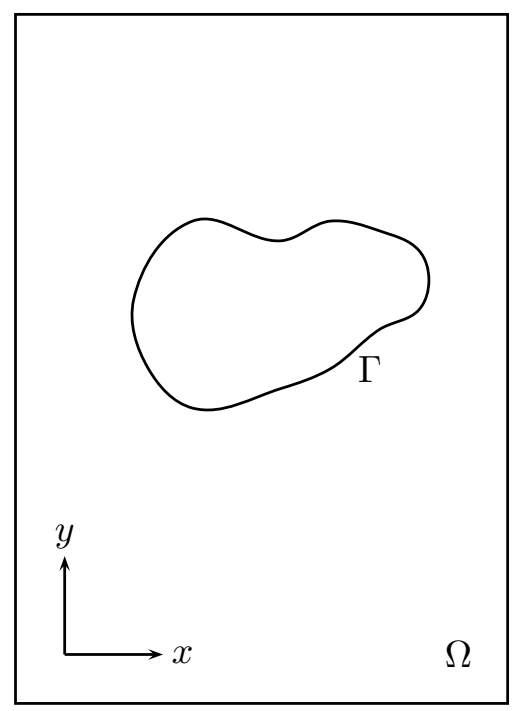

(a)

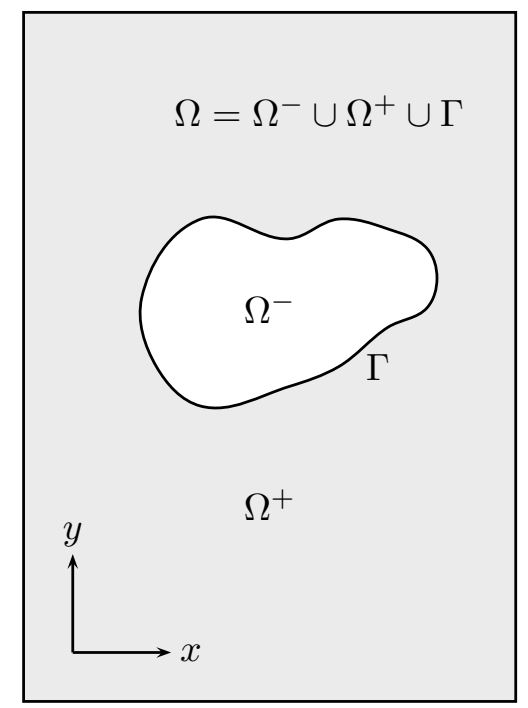

(b)

Figura 2.1: (a) Interface $\Gamma$ em um domínio euleriano $\Omega$. (b) Fase dispersa, $\Omega^{-}$, fase contínua, $\Omega^{+}$.

O Modelo de Um Fluido [32, 105, 106, 107] requer a definição de uma função indicadora de fluidos para discernir as diferentes fases entre si: uma, a "fase dispersa", contida no interior da região delimitada pela interface de separação dada pela curva fechada simples, $\Gamma$. Esta região denotaremos por $\Omega^{-}$. A outra, a "fase contínua", está definida no exterior e denotaremos por $\Omega^{+}=\Omega-\Omega^{-}$. Note que $\Omega^{-} \cap \Omega^{+}=\emptyset$ e que $\Gamma=\partial \Omega^{-}=\partial \Omega^{+}$, onde $\partial \Omega^{-}$e $\partial \Omega^{+}$representam o contorno de $\Omega^{-}$e $\Omega^{+}$, respectivamente. A representação da fase dispersa e da fase contínua é mostrada na Figura 2.1(b).

Tipicamente, construimos uma função indicadora de fluidos $\phi$ que satisfaz as propriedades:

1. ser pelo menos contínua em $\Omega$ e é tal que

2. $\phi<0$ em $\Omega^{-}, \phi=0$ sobre $\Gamma$ e $\phi>0$ em $\Omega^{+}$.

A Figura 2.2(a) mostra a representação da função indicadora $\phi$. Existem diversas formas de se obter esta função, que vão desde a aplicação de Geometria Computacional [31] até a solução de uma equação diferencial parcial (e.g. elíptica [92, 107] e hiperbólica [73, 94]). 
Para fluidos bifásicos nos quais não há mistura e cada fase individualmente mantém suas propriedades constantes sobre linhas de corrente, podemos substituir a equação (2.2) por

$$
\nabla \cdot \mathbf{u}=0
$$

A posição da interface, supostamente conhecida, é empregada para atualizar as propriedades materiais por intermédio de $\phi$ (lembremos que estamos com um Método de Acompanhamento de Fronteira - Front-tracking Method). A ideia aqui é solucionar um só conjunto de equações que modelem o escoamento [32, 73, 106, 107].

A Função de Heaviside que depende da função indicadora $\phi$, dada por

$$
H(\phi)= \begin{cases}0, & \text { se } \phi \leq 0 \\ 1, & \text { se } \phi>0\end{cases}
$$

é usada para atualizar as propriedades materiais das fases que compõem o fluido [31, 32, 73, 106]. Escolhemos esta função por que ela se anula na fase dispersa $(\phi<0)$ e é igual a 1 na fase contínua $(\phi>0)$, como mostra a Figura 2.2(b). Mais detalhes sobre a Função de Heaviside podem ser encontradas em [73, 106].

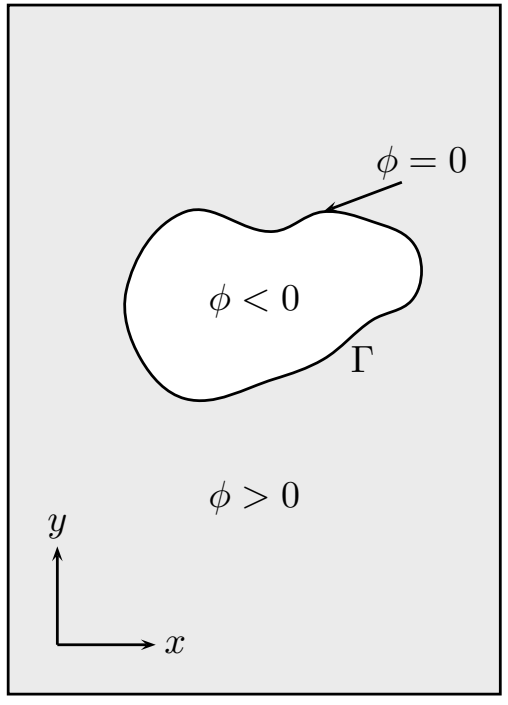

(a)

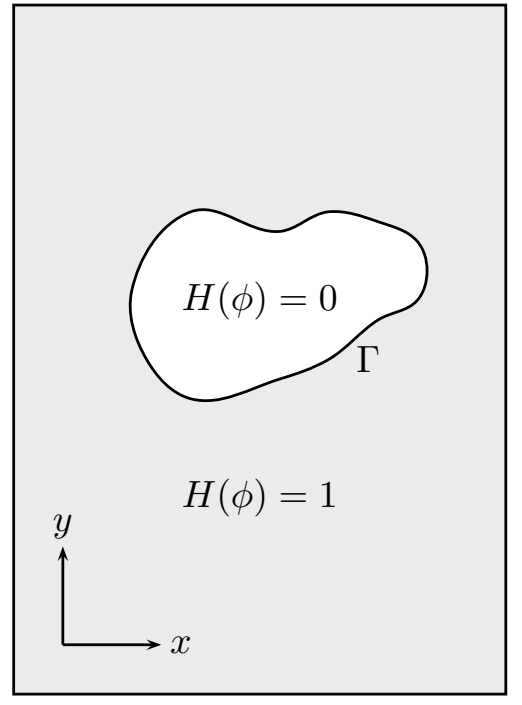

(b)

Figura 2.2: (a) Função indicadora, positiva para fase contínua e negativa para fase dispersa. (b) Função de Heaviside: o valor 1 representa a fase contínua e 0 a fase dispersa.

Suponhamos que $\rho^{-}$e $\mu^{-}$e que $\rho^{+}$e $\mu^{+}$denotem respectivamente a massa específica e a viscosidade dinâmica da fase dispersa e da fase contínua. Com o auxílio de (2.4), podemos definir a massa específica e a viscosidade dinâmica para cada ponto do domínio como

$$
\begin{gathered}
\rho(\phi)=\rho^{-}+\left(\rho^{+}-\rho^{-}\right) H(\phi), \\
\mu(\phi)=\mu^{-}+\left(\mu^{+}-\mu^{-}\right) H(\phi) .
\end{gathered}
$$


Pelas considerações anteriores, passamos de (2.1)-(2.2), para um modelo matemático dado por

$$
\begin{aligned}
\rho(\phi)\left[\frac{\partial \mathbf{u}}{\partial t}+(\mathbf{u} \cdot \nabla) \mathbf{u}\right] & =-\nabla p+\nabla \cdot\left[\mu(\phi)\left(\nabla \mathbf{u}+\nabla \mathbf{u}^{T}\right)\right]+\alpha_{p} \nabla \cdot \mathbf{S}(\phi)+\mathbf{f}, \\
\nabla \cdot \mathbf{u} & =0 .
\end{aligned}
$$

\subsection{Dinâmica da interface de separação}

No Modelo de Um Fluido [32, 105, 106, 107], o movimento da interface e a influência da tensão superficial no escoamento, contabilizada por uma parcela que compõe o termo forçante $\mathbf{f}$ em (2.7), são feitos como no Método da Fronteira Imersa proposto por Peskin [68, 81]. A força de tensão superficial na interface é "espalhada" ao fluido por intermédio da expressão

$$
\mathbf{f}_{\mathbf{s}}(\mathbf{x}, t)=\int_{\Gamma} \mathbf{F}(\alpha, t) \delta(\mathbf{x}-\mathbf{X}(\alpha, t)) d \alpha,
$$

onde $\mathbf{F}(\alpha, t)$ é a densidade de força da interface, $\mathbf{X}(\alpha, t)$ são os pontos da interface, $\alpha$ é o parâmetro lagrangiano e $\mathbf{x}$ pertence ao domínio euleriano. Na Figura 2.3, apresentamos uma representação para cada uma destas variáveis.

A posição dos pontos lagrangianos que compõem a interface de separação (fronteira imersa) é obtida pela solução da equação diferencial ordinária proveniente da condição de não deslizamento (no-slip condition) aplicada a eles,

$$
\frac{\partial \mathbf{X}((\alpha, t), t)}{\partial t}=\mathbf{U}(\mathbf{X}(\alpha, t), t)
$$

onde

$$
\mathbf{U}(\mathbf{X}(\alpha, t), t) \doteq \mathbf{u}(\mathbf{X}(\alpha, t), t)=\int_{\Omega} \mathbf{u}(\mathbf{x}, t) \delta(\mathbf{x}-\mathbf{X}(\alpha, t)) d \mathbf{x}
$$

A expressão (2.10) define a operação de "interpolação" da velocidade euleriana do escoamento, $\mathbf{u}$, para os pontos da interface, resultando na velocidade lagrangiana dos pontos da interface.

Para determinar a densidade de força interfacial temos duas formulações: a formulação clássica [81, 85, 106], que determina a força interfacial diretamente sobre o domínio lagrangiano e depois a espalha para o domínio euleriano empregando a proposta original do Método de Fronteira Imersa (2.9), e a formulação híbrida [32, 92], que define a densidade de força da interface com uma formulação puramente euleriana dependente da função indicadora de fluido, seguindo a ideia dos métodos de captura de interface.

Na formulação clássica, isto é, como originalmente proposta por Peskin [81, 85, 106], a expressão para determinar a densidade de força aplicada pela interface no domínio euleriano é dada por

$$
\begin{aligned}
\mathbf{F} & =\frac{\partial(\sigma \mathbf{t})}{\partial \alpha}=\frac{\partial \sigma}{\partial \alpha} \mathbf{t}+\sigma \frac{\partial \mathbf{t}}{\partial \alpha} \\
& =\frac{\partial \sigma}{\partial \alpha} \mathbf{t}+\sigma\left\|\frac{\partial \mathbf{X}}{\partial \alpha}\right\| \kappa \mathbf{n},
\end{aligned}
$$

onde $\sigma$ é a tensão superficial, $\kappa=\left\|\frac{\partial \mathbf{t}}{\partial \alpha}\right\| /\left\|\frac{\partial \mathbf{X}}{\partial \alpha}\right\|$ é a curvatura da interface, $\mathbf{t}=\frac{\partial \mathbf{X}}{\partial \alpha} /\left\|\frac{\partial \mathbf{X}}{\partial \alpha}\right\|$ é o vetor tangente unitário à direção da interface e $\mathbf{n}=\frac{\partial \mathbf{t}}{\partial \alpha} /\left\|\frac{\partial \mathbf{t}}{\partial \alpha}\right\|$ é o vetor normal unitário à interface 


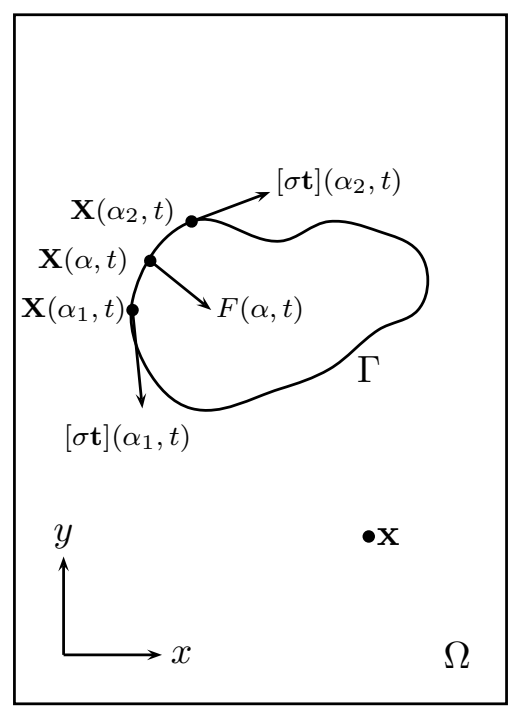

Figura 2.3: Representação de pontos no dominio euleriano e lagrangiano. O ponto $\mathrm{x}$ se encontra no domínio euleriano. Os pontos $\mathbf{X}\left(\alpha_{i}, t\right)$ pertencem ao dominio lagrangiano com $\alpha_{i} \in \alpha$.

(Figura 2.3). Quando o coeficiente de tensão superficial $\sigma$ é constante e $\left\|\frac{\partial \mathbf{X}}{\partial \alpha}\right\|=1$, temos que

$$
\mathbf{F}=\sigma \kappa \mathbf{n}
$$

Logo, para $\sigma$ constante, de (2.9) e (2.13) temos a formulação clássica para a força de tensão superficial (via acompanhamento de interface) dada por

$$
\mathbf{f}_{s}=\sigma \int_{\Gamma} \kappa(\alpha) \delta(\mathbf{x}-\mathbf{X}(\alpha, t)) \mathbf{n}(\alpha) d \alpha .
$$

Por outro lado, lembrando de técnicas de captura de interface [74, 92], uma forma alternativa para calcular a força oriunda da tensão superficial, de maneira puramente euleriana e usando uma função indicadora de fluido $\phi$, é dada por

$$
\mathbf{f}_{e}(\mathbf{x})=\sigma \kappa_{e}(\mathbf{x}) \nabla H(\phi(\mathbf{x}))
$$

onde $\kappa_{e}=-\nabla \cdot \frac{\nabla \phi}{|\nabla \phi|}$ é a curvatura euleriana e $H$ é a Função de Heaviside (2.4).

Seguindo [92], há ainda uma terceira maneira para obtermos a força de tensão superficial a partir de uma formulação híbrida. Denotada por $\mathbf{f}_{h}$ ela se escreve como

$$
\mathbf{f}_{h}(\mathbf{x})=\sigma \kappa_{L}(\mathbf{x}) \nabla H(\phi(\mathbf{x}))
$$

onde

$$
\kappa_{L}(\mathbf{x})=\frac{1}{\sigma} \frac{\mathbf{f}_{s}(\mathbf{x}) \cdot \mathbf{n}_{L}(\mathbf{x})}{\mathbf{n}_{L}(\mathbf{x}) \cdot \mathbf{n}_{L}(\mathbf{x})}, \quad \operatorname{com} \quad \mathbf{n}_{L}(\mathbf{x})=\int_{\Gamma} \delta(\mathbf{x}-\mathbf{X}(\alpha, t)) \mathbf{n}(\alpha) d \alpha
$$

Consideramos aqui que uma das fases poderá ser viscoelástica (não newtoniana). Os modelos que empregaremos influenciarão o escoamento pela ação do termo $\nabla \cdot \mathbf{S}(\phi)$ em (2.7). Se o escoamento for puramente newtoniano, escolheremos $\alpha_{p}$ como sendo zero. No que segue, detalharemos os modelos 
matemáticos considerados para a fase viscoelástica.

\subsection{Modelagem matemática da fase viscoelástica}

O tensor $\mathbf{S}$, assumido simétrico, permite caracterizar os fluidos como newtonianos, quando $\nabla \cdot \mathbf{S}$ é nulo, ou como não newtonianos no caso contrário. No caso bifásico definimos $\mathbf{S}$ como sendo

$$
\mathbf{S}(\phi)=I+\left(\mathbf{S}_{\mathbf{p}}-I\right) H(\phi)
$$

onde $\mathbf{S}_{\mathbf{p}}$ é o tensor para a fase viscoelástica. Uma representação esquemática desta situação pode ser observada na Figura 2.4 .

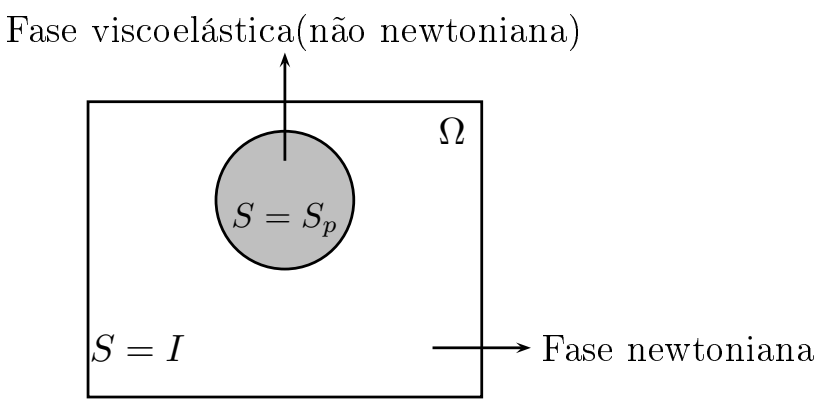

Figura 2.4: Definição do tensor $S$ no caso bifásico com uma fase viscoelástica (em cinza).

Um dos modelos matemáticos mais simples para representarmos fluidos viscoelásticos é o Modelo Oldroyd-B [60, 99, 100, 101, 103], o qual se escreve como

$$
\tau_{p} \mathbf{S}_{\mathbf{p}}^{\nabla}=-\left(\mathbf{S}_{\mathbf{p}}-G I\right)
$$

onde, como antes, $\mathbf{S}_{\mathbf{p}}$ é o tensor viscoelástico, $\tau_{p}$ é a escala de tempo de relaxação do polímero, $G$ é o estresse isotrópico induzido pelo campo viscoelástico na ausência de fluido e

$$
\mathbf{S}_{\mathbf{p}} \nabla=\frac{\partial \mathbf{S}_{\mathbf{p}}}{\partial t}+\mathbf{u} \cdot \nabla \mathbf{S}_{\mathbf{p}}-\left(\nabla \mathbf{u} \mathbf{S}_{\mathbf{p}}+\mathbf{S}_{\mathbf{p}} \nabla \mathbf{u}^{T}\right)
$$

é a derivada convectada superior.

O Modelo Oldroyd-B descreve uma relação entre o gradiente da velocidade do fluido $(\nabla \mathbf{u})$, as propriedades poliméricas e o tensor viscoelástico e pode ser proposto a partir de considerações a nível microscópico: vem da aplicação da Lei de Hooke assumindo-se que as cadeias poliméricas sejam representadas por molas que podem ser estendidas infinitamente. Devido a esta hipótese, este modelo é bastante criticado. Na literatura, encontramos outros modelos que também aproximam o tensor $\mathbf{S}$ por molas mas para os quais a extensão das cadeias poliméricas são finitas. É o caso do Modelo FENE-P, o qual é uma modificação do Modelo FENE (Finitely Extensible Nonlinear 
Elastic) proposta por Peterlin [19]. No Modelo FENE-P, a expressão para o tensor é dada por

$$
\begin{aligned}
\tau_{p} \mathbf{C}^{\nabla} & =-\left(\mathbf{S}_{\mathbf{p}}-G I\right), \\
\mathbf{S}_{\mathbf{p}} & =\frac{\mathbf{C}}{1-\left(\operatorname{tr}(\mathbf{C}) / l^{2}\right)},
\end{aligned}
$$

sendo $l$ um parâmetro relacionado à extensibilidade média máxima das cadeias poliméricas e $\mathbf{C}$ um tensor intermediário para determinar o tensor $\mathbf{S}_{p}$.

Um valor adimensional importante no estudo do comportamento dos Métodos Oldroyd-B e FENE-P, é o número de Weissenberg, denotado por $W_{i}$, o qual é dado pela razão entre o tempo de relaxação polimérica e o tempo característico do fluido. Mais detalhes no Apêndice A.

\subsubsection{Detalhamento do Modelo Oldroyd-B}

Seja

$$
\mathbf{S}=\left[\begin{array}{ll}
\mathbf{S}_{x x} & \mathbf{S}_{x y} \\
\mathbf{S}_{x y} & \mathbf{S}_{y y}
\end{array}\right]
$$

a representação matricial do tensor viscoelástico simétrico e bidimensional, onde $S_{x x}, S_{x y}$ e $S_{y y}$ são suas componentes tensoriais.

Consideremos primeiramente as expressões

$$
\begin{gathered}
\nabla \mathbf{u} \cdot \mathbf{S}=\left[\begin{array}{ll}
\frac{\partial u}{\partial x} S_{x x}+\frac{\partial u}{\partial y} S_{x y} & \frac{\partial u}{\partial x} S_{x y}+\frac{\partial u}{\partial y} S_{y y} \\
\frac{\partial v}{\partial x} S_{x x}+\frac{\partial v}{\partial y} S_{x y} & \frac{\partial v}{\partial x} S_{x y}+\frac{\partial v}{\partial y} S_{y y}
\end{array}\right] \\
\mathbf{S} \cdot \nabla \mathbf{u}^{T}=\left[\begin{array}{ll}
\frac{\partial u}{\partial x} S_{x x}+\frac{\partial u}{\partial y} S_{x y} & \frac{\partial v}{\partial x} S_{x x}+\frac{\partial v}{\partial y} S_{x y} \\
\frac{\partial u}{\partial x} S_{x y}+\frac{\partial u}{\partial y} S_{y y} & \frac{\partial v}{\partial x} S_{x y}+\frac{\partial v}{\partial y} S_{y y}
\end{array}\right]
\end{gathered}
$$

Pela equação da continuidade (2.3), temos $\frac{\partial u}{\partial x}+\frac{\partial v}{\partial y}=0$ e, assim

$$
\nabla \mathbf{u} \cdot \mathbf{S}+\mathbf{S} \cdot \nabla \mathbf{u}^{T}=\left[\begin{array}{cc}
2\left(\frac{\partial u}{\partial x} S_{x x}+\frac{\partial u}{\partial y} S_{x y}\right) & \frac{\partial v}{\partial x} S_{x x}+\frac{\partial u}{\partial y} S_{y y} \\
\frac{\partial v}{\partial x} S_{x x}+\frac{\partial u}{\partial y} S_{y y} & 2\left(\frac{\partial v}{\partial x} S_{x y}+\frac{\partial v}{\partial y} S_{y y}\right)
\end{array}\right]
$$

Da expressão (2.24) e ao substituirmos a forma matricial de $\mathbf{S}$ (2.23) na forma adimensional do Modelo Oldroyd-B

$$
\mathbf{S}^{\nabla}=-W_{i}^{-1}(\mathbf{S}-I)
$$

tal que

$$
\frac{\partial \mathbf{S}}{\partial t}+\mathbf{u} \cdot \nabla \mathbf{S}-\left(\nabla \mathbf{u S}+\mathbf{S} \nabla \mathbf{u}^{T}\right)=-W_{i}^{-1}(\mathbf{S}-I),
$$


obtemos para cada uma das componentes tensoriais o sistema de equações

$$
\begin{aligned}
& \frac{\partial \mathbf{S}_{x x}}{\partial t}+u \frac{\partial \mathbf{S}_{x x}}{\partial x}+v \frac{\partial \mathbf{S}_{x x}}{\partial y}-2\left(\frac{\partial u}{\partial x} \mathbf{S}_{x x}+\frac{\partial u}{\partial y} \mathbf{S}_{x y}\right)=-W_{i}^{-1}\left(\mathbf{S}_{x x}-1\right) \\
& \frac{\partial \mathbf{S}_{y y}}{\partial t}+u \frac{\partial \mathbf{S}_{y y}}{\partial x}+v \frac{\partial \mathbf{S}_{y y}}{\partial y}-2\left(\frac{\partial v}{\partial x} \mathbf{S}_{x y}+\frac{\partial v}{\partial y} \mathbf{S}_{y y}\right)=-W_{i}^{-1}\left(\mathbf{S}_{y y}-1\right) \\
& \frac{\partial \mathbf{S}_{x y}}{\partial t}+u \frac{\partial \mathbf{S}_{x y}}{\partial x}+v \frac{\partial \mathbf{S}_{x y}}{\partial y}-\frac{\partial v}{\partial x} \mathbf{S}_{x x}-\frac{\partial u}{\partial y} \mathbf{S}_{y y}=-W_{i}^{-1} \mathbf{S}_{x y}
\end{aligned}
$$

que podem ser reescritas como

$$
\begin{gathered}
\frac{\partial \mathbf{S}_{x x}}{\partial t}=g_{x x}(\mathbf{S}, \mathbf{u}), \\
\frac{\partial \mathbf{S}_{y y}}{\partial t}=g_{y y}(\mathbf{S}, \mathbf{u}), \\
\frac{\partial \mathbf{S}_{x y}}{\partial t}=g_{x y}(\mathbf{S}, \mathbf{u}),
\end{gathered}
$$

onde

$$
\begin{aligned}
& g_{x x}(\mathbf{S}, \mathbf{u})=-\left(u \frac{\partial \mathbf{S}_{x x}}{\partial x}+v \frac{\partial \mathbf{S}_{x x}}{\partial y}\right)+2\left(\frac{\partial u}{\partial x} \mathbf{S}_{x x}+\frac{\partial u}{\partial y} \mathbf{S}_{x y}\right)-W_{i}^{-1}\left(\mathbf{S}_{x x}-1\right), \\
& g_{y y}(\mathbf{S}, \mathbf{u})=-\left(u \frac{\partial \mathbf{S}_{y y}}{\partial x}+v \frac{\partial \mathbf{S}_{y y}}{\partial y}\right)+2\left(\frac{\partial v}{\partial x} \mathbf{S}_{x y}+\frac{\partial v}{\partial y} \mathbf{S}_{y y}\right)-W_{i}^{-1}\left(\mathbf{S}_{y y}-1\right) \\
& g_{x y}(\mathbf{S}, \mathbf{u})=-\left(u \frac{\partial \mathbf{S}_{x y}}{\partial x}+v \frac{\partial \mathbf{S}_{x y}}{\partial y}\right)+\frac{\partial v}{\partial x} \mathbf{S}_{x x}+\frac{\partial u}{\partial y} \mathbf{S}_{y y}-W_{i}^{-1} \mathbf{S}_{x y} .
\end{aligned}
$$

Se definirmos

$$
\mathbf{g}(\mathbf{S}, \mathbf{u})=\left[\begin{array}{ll}
g_{x x}(\mathbf{S}, \mathbf{u}) & g_{x y}(\mathbf{S}, \mathbf{u}) \\
g_{x y}(\mathbf{S}, \mathbf{u}) & g_{y y}(\mathbf{S}, \mathbf{u})
\end{array}\right],
$$

o tensor $\mathbf{S}$ (2.27) é a solução de um sistema de equações diferenciais ordinárias da forma

$$
\frac{\partial \mathbf{S}}{\partial t}=\mathbf{g}(\mathbf{S}, \mathbf{u})
$$

cuja solução aproximada pode ser obtida por diferentes métodos. Na Seção 3.4 se encontra a solução que usamos neste trabalho.

Detalhes adicionais sobre a forma adimensional do Modelo Oldroyd-B encontram-se no Apêndice A.

\subsubsection{Detalhamento do Modelo FENE-P}

Como mencionamos, uma desvantagem do Modelo Oldroyd-B é que ele permite o alongamento infinito das cadeias poliméricas, o que não acomoda bem a realidade física dos polímeros e pode acontecer um grande aumento do tensor viscoelástico. Peterlin, propôs em 1961 um modelo com uma aproximação finita para as cadeias poliméricas com o uso de aproximações estatísticas do Modelo FENE "finitely extensible nonlinear elastic", conhecido como o modelo FENE-P. Este modelo está 
dado na forma adimensional por

$$
\begin{aligned}
W_{i} \mathbf{C}^{\nabla} & =-(\mathbf{S}-I), \\
\mathbf{S} & =\frac{\mathbf{C}}{1-\left(\operatorname{tr}(\mathbf{C}) / l^{2}\right)},
\end{aligned}
$$

sendo $l$ um parâmetro relacionado com a extensibilidade média máxima das cadeias poliméricas.

Para que este modelo seja bem definido, devemos determinar o valor inicial $\mathbf{C}^{(0)}$ que corresponde ao tensor $\mathbf{C}$, pois o valor inicial conhecido $\mathbf{S}^{(0)}$ corresponde ao tensor $\mathbf{S}$.

Para obtermos o valor de $\mathbf{C}^{(0)}$, partimos da equação (2.32) e isolamos nela $\mathbf{C}$, obtendo

$$
\mathbf{C}=\frac{l^{2}-\operatorname{tr}(\mathbf{C})}{l^{2}} \mathbf{S}
$$

Aplicando o traço nos dois lados da Equação (2.33), temos que

$$
\begin{aligned}
\operatorname{tr}(\mathbf{C}) & =\frac{l^{2}-\operatorname{tr}(\mathbf{C})}{l^{2}} \operatorname{tr}(\mathbf{S}) \\
& =\operatorname{tr}(\mathbf{S})-\frac{\operatorname{tr}(\mathbf{C}) \operatorname{tr}(\mathbf{S})}{l^{2}}
\end{aligned}
$$

e

$$
\operatorname{tr}(\mathbf{S})=\operatorname{tr}(\mathbf{C})\left(1+\frac{\operatorname{tr}(\mathbf{S})}{l^{2}}\right)
$$

Ao isolar o $\operatorname{tr}(\mathbf{C})$ na Equação (2.34), obtemos

$$
\operatorname{tr}(\mathbf{C})=\frac{\operatorname{tr}(\mathbf{S})}{1+\left(\operatorname{tr}(\mathbf{S}) / l^{2}\right)}
$$

Finalmente, substituindo (2.35) em (2.33) estabelecemos uma relação para o tensor $\mathbf{C}$ dependente do tensor $\mathbf{S}$, dada por

$$
\begin{aligned}
\mathbf{C} & =\mathbf{S}\left(\frac{l^{2}-\frac{\operatorname{tr}(\mathbf{S})}{1+\left(\operatorname{tr}(\mathbf{S}) / l^{2}\right)}}{l^{2}}\right) \\
& =\mathbf{S} \frac{l^{2}-\frac{l^{2} \operatorname{tr}(\mathbf{S})}{l^{2}+\operatorname{tr}(\mathbf{S})}}{l^{2}} \\
& =\mathbf{S} \frac{l^{2}}{l^{2}+\operatorname{tr}(\mathbf{S})}, \\
& =\frac{\mathbf{S}}{1+\left(\operatorname{tr}(\mathbf{S}) / l^{2}\right)} .
\end{aligned}
$$

Da expressão (2.36), temos que a condição inicial para o tensor $\mathbf{C}$ é dada por

$$
\mathbf{C}^{(0)}=\frac{\mathbf{S}^{(0)}}{1+\left(\operatorname{tr}\left(\mathbf{S}^{(0)}\right) / l^{2}\right)} .
$$

Os detalhes da solução matemática deste modelo são equivalentes aos feitos para o Modelo 
Oldroyd-B na Seção 2.3.1, dado que podemos expressar o Modelo FENE-P de uma forma equivalente à dada na expressão (2.30) para o Modelo Oldroyd-B. Assim, escrevemos

$$
\frac{\partial \mathbf{C}}{\partial t}=\mathbf{h}(\mathbf{C}, \mathbf{S}, \mathbf{u})
$$

onde

$$
\mathbf{h}(\mathbf{C}, \mathbf{S}, \mathbf{u})=-\mathbf{u} \cdot \nabla \mathbf{S}+\left(\nabla \mathbf{u} \mathbf{S}+\mathbf{S} \nabla \mathbf{u}^{T}\right)-W_{i}^{-1}(\mathbf{S}-I)
$$

A forma como trabalhamos o modelo do tensor viscoelástico bidimensional, nesta e na seção anterior facilita a extensão para o modelo tridimensional.

\subsection{Adimensionalização do modelo matemático}

A seguir, apresentamos a forma adimensional das equações (2.7), (2.8) e (2.19). Os detalhes para a adimensionalização estão no Apêndice A. Escolhendo valores característicos de velocidade, comprimento e tempo, dentro outros, definimos

$$
\overline{\mathbf{u}}=\frac{\mathbf{u}}{U_{c}}, \overline{\mathbf{x}}=\frac{\mathbf{x}}{L_{c}}, \bar{t}=\frac{t}{t_{c}}, \bar{p}=\frac{p L_{c}}{\mu_{c} U_{c}}, \bar{\mu}=\frac{\mu}{\mu_{c}}, \bar{\rho}=\frac{\rho}{\rho_{c}}, \overline{\mathbf{S}}_{p}=\frac{\mathbf{S}_{\mathbf{p}}}{G} \quad \text { e } \quad \overline{\mathbf{S}}(\phi)=I+\left(\overline{\mathbf{S}}_{\mathbf{p}}-I\right) H(\phi),
$$

onde $\overline{\mathbf{u}}=(\bar{u}, \bar{v})$ e $\overline{\mathbf{x}}=(\bar{x}, \bar{y}), L_{c}$ é o comprimento característico dado pelo comprimento do domínio, $U_{c}$ é a velocidade característica, $t_{c}$ é o tempo característico requerido para o fluido ser transportado a uma distância da ordem do comprimento do domínio $\left(t_{c}=\frac{L}{U_{c}}\right), \mu_{c}$ é a viscosidade característica e $\mathbf{S}_{p}$ é o tensor na fase viscoelástica.

O modelo adimensional que obtemos está dado por

$$
\begin{gathered}
\operatorname{Re} \bar{\rho}(\phi)\left[\frac{\partial \overline{\mathbf{u}}}{\partial \bar{t}}+\left(\overline{\mathbf{u}} \cdot \nabla^{\prime}\right) \overline{\mathbf{u}}\right]=-\nabla^{\prime} \bar{p}+\nabla^{\prime}\left[\bar{\mu}(\phi)\left(\nabla^{\prime} \overline{\mathbf{u}}+\nabla^{\prime} \overline{\mathbf{u}}^{T}\right)\right]+\alpha_{p} \beta \nabla^{\prime} \cdot \overline{\mathbf{S}}(\phi)+\overline{\mathbf{f}} \\
\nabla^{\prime} \cdot \overline{\mathbf{u}}=0
\end{gathered}
$$

onde $R e=\frac{\rho_{c} U_{c} L_{c}}{\mu_{c}}$ é o número de Reynolds, $\overline{\mathbf{f}}=\mathbf{f} \frac{L_{c}^{2}}{\mu_{c} U_{c}^{2}}$ e $\beta=G \frac{t_{c}}{\mu_{c}}$. A equação adimensional para o modelo Oldroyd-B (2.19), é dada por

$$
W_{i} \overline{\mathbf{S}}_{p}^{\nabla}=-\left(\overline{\mathbf{S}}_{p}-I\right)
$$

onde $W_{i}=\frac{\tau_{p}}{\tau_{c}}$ é o número de Weissenberg.

Nas aplicações em que o número de Reynolds é muito pequeno, isto é $R e<<1$, podemos obter a partir das equações adimensionais (2.40) a equação de Stokes estacionária

$$
-\nabla^{\prime} \bar{p}+\nabla^{\prime}\left[\bar{\mu}(\phi)\left(\nabla^{\prime} \overline{\mathbf{u}}+\nabla^{\prime} \overline{\mathbf{u}}^{T}\right)\right]+\alpha_{p} \beta \nabla^{\prime} \cdot \overline{\mathbf{S}}(\phi)+\overline{\mathbf{f}}=0
$$

Um dos nossos objetivos são as aplicações a baixos número de Reynolds e, por isso, nossa preocupação com modelos matemáticos adequados a estas situações como as equações de Stokes. Observe que a equação de Stokes (2.43) independe do tempo de maneira explícita. O avanço no 
tempo é forçado pelo tensor viscoelástico e pela força da fronteira imersa no fluido. Uma metodologia numérica para obter a solução das equações de Stokes será estudada no Capítulo 3.

Para facilitar daqui em diante, quando nos referirmos à forma adimensional de alguma das equações, suprimiremos os símbolos " - " e " " " que as representam.

A equação (2.43) não depende da densidade $\rho$, mas o número de Reynolds $R e$ precisa dela para ser determinado e a equação (2.43) é válida para Re pequeno.

Quando a viscosidade $\mu$ é constante, temos que $\mu_{c}=\mu$ e $\bar{\mu}=1$. Neste caso, a equação (2.43) é dada por

$$
-\nabla p+\nabla^{2} \mathbf{u}=\mathbf{f}
$$

onde $\mathbf{f}=-\alpha_{p} \beta \nabla \cdot \mathbf{S}(\phi)-\mathbf{f}_{s}$.

\subsection{Modelos matemáticos resultantes}

As equações apresentadas até aqui modelam a dinâmica de escoamentos incompressíveis transientes e estacionários de fluidos bifásicos, os quais podem conter uma interface imersa e uma fase viscoelástica. Segue um resumo dos modelos matemáticos.

\subsubsection{Escoamentos incompressíveis transientes de fluidos bifásicos}

$$
\begin{aligned}
\rho(\phi)\left[\frac{\partial \mathbf{u}}{\partial t}+(\mathbf{u} \cdot \nabla) \mathbf{u}\right] & =-\nabla p+\nabla \cdot\left[\mu(\phi)\left(\nabla \mathbf{u}+\nabla \mathbf{u}^{T}\right)\right]+\alpha_{p} \nabla \cdot \mathbf{S}(\phi)+\mathbf{f}, \\
\nabla \cdot \mathbf{u} & =0 \\
\mathbf{f}(\mathbf{x}, t) & =\int_{\Gamma} \mathbf{F}(\alpha, t) \delta(\mathbf{x}-\mathbf{X}(\alpha, t)) d \alpha \\
\frac{\partial \mathbf{X}(\alpha, t)}{\partial t} & =\int_{\Omega} \mathbf{u}(\mathbf{x}, t) \delta(\mathbf{x}-\mathbf{X}(\alpha, t)) d \mathbf{x} \\
\mathbf{S}(\phi) & =I+\left(\mathbf{S}_{\mathbf{p}}-I\right) H(\phi)
\end{aligned}
$$

\subsubsection{Força interfacial}

Definimos duas opções para determinar a força interfacial com tensão superficial $\sigma$ constante, dadas por:

- Formulação clássica

$$
\mathbf{f}(\mathbf{x})=\mathbf{f}_{s}(\mathbf{x})=\sigma \int \kappa(\alpha) \delta(\mathbf{x}-\mathbf{X}(\alpha, t)) \mathbf{n}(\alpha) d \alpha
$$

- Formulação híbrida

$$
\mathbf{f}(\mathbf{x})=\mathbf{f}_{h}(\mathbf{x})=\sigma \kappa_{L}(\mathbf{x}) \nabla H(\phi(\mathbf{x})) .
$$

As variáveis $\kappa(\alpha), \mathbf{n}(\alpha)$ e $\kappa_{L}(\mathbf{x})$ foram definidas em (2.12) e (2.17). 


\subsubsection{Tensor viscoelástico}

Para determinar o tensor viscoelástico $\mathbf{S}_{\mathbf{p}}$, definimos dois modelos:

- Modelo Oldroyd-B

$$
\tau_{p} \mathbf{S}_{\mathbf{p}}^{\nabla}=-\left(\mathbf{S}_{\mathbf{p}}-G I\right)
$$

- Modelo FENE-P

$$
\begin{aligned}
\tau_{p} \mathbf{C}^{\nabla} & =-\left(\mathbf{S}_{\mathbf{p}}-G I\right), \\
\mathbf{S}_{\mathbf{p}} & =\frac{\mathbf{C}}{1-\left(\operatorname{tr} \mathbf{C} / l^{2}\right)},
\end{aligned}
$$

onde $\tau_{p}, G$ e $l$ foram definidos em (2.19) e (2.22).

2.5.4 Escoamentos incompressíveis estacionários de fluidos bifásicos

$$
\begin{aligned}
-\nabla p+\nabla\left[\mu(\phi)\left(\nabla \mathbf{u}+\nabla \mathbf{u}^{T}\right)\right] & =-\mathbf{f}-\alpha_{p} \beta \nabla \cdot \mathbf{S}(\phi), \\
\nabla \cdot \mathbf{u} & =0, \\
\mathbf{f}(\mathbf{x}, t) & =\int \mathbf{F}(\alpha, t) \delta(\mathbf{x}-\mathbf{X}(\alpha, t)) d \alpha, \\
\frac{\partial \mathbf{X}(\alpha, t)}{\partial t} & =\int_{\Omega} \mathbf{u}(\mathbf{x}, t) \delta(\mathbf{x}-\mathbf{X}(\alpha, t)) d \mathbf{x}, \\
\mathbf{S}(\phi) & =I+\left(\mathbf{S}_{\mathbf{p}}-I\right) H(\phi) .
\end{aligned}
$$




\section{Capítulo 3}

\section{Metodologia numérica: discretizações no tempo e no espaço (malha uniforme)}

Neste Capítulo, descrevemos as discretizações no tempo e no espaço adotadas para o modelo matemático proposto no Capítulo 2. No espaço, apresentamos aqui a discretização em uma malha uniforme com o intuito de simplificar a exposição. No capítulo subsequente, descrevemos as alterações e particularidades requeridas para a extensão da discretização espacial para uma malha bloco-estruturada composta por refinamentos localizados (Structured Adaptive Mesh Refinement Grid - SAMR grid). Nossa motivação é a simulação de escoamentos incompressíveis de fluidos bifásicos newtonianos e não newtonianos (viscoelásticos) com estruturas neles imersas.

Na Seção 3.1, apresentamos as aproximações associadas ao domínio espacial. Isto inclui as discretizações dos domínios euleriano e lagrangiano e, neles, as dos operadores diferenciais espaciais. Empregaremos o Método de Diferenças Finitas. Além disso, apresentamos brevemente como obtemos a função indicadora de fluidos.

A metodologia numérica para a solução de escoamentos incompressíveis bifásicos transientes é descrita na Seção 3.2. Para tais escoamentos, modelados pelas equações de Navier-Stokes, empregamos o Método da Projeção de Chorin-Temam [34, 97]. Baseado no Teorema da Decomposição de Hodge [35], tal método desacopla a velocidade da pressão. Em conjunto com o Método da Projeção de Chorin-Temam, para a discretização no tempo empregamos uma metodologia implícita-explícita (IMEX) [5, 32, 112]. Na Seção 3.3, apresentamos a discretização das equações do movimento da interface de separação entre as fases.

A aproximação do tensor viscoelástico é descrita na Seção 3.4. Nos modelos Oldroyd-B e FENE$\mathrm{P}$ [19, 60, 99, 103], a dinâmica temporal é descrita, a cada passo no tempo, pela derivada convectada superior. Na discretização do tensor viscoelástico, empregamos no tempo um esquema do tipo "TVD" (Total Variation Diminishing), dado por um Método de Runge-Kutta de segunda ordem [24], e no espaço, o termo advectivo tem sua discretização dada por um esquema "ENO" de terceira ordem (Essentially Non-Oscillatory scheme) [9, 73, 91, 93].

A metodologia numérica que empregamos para os escoamentos estacionários de Stokes (escoamentos a Reynolds zero, $R e \rightarrow 0$ ) é descrita na Seção 3.5. Como o termo forçante desta equação depende do tempo, é necessário resolvê-la a todo instante de tempo. Empregamos, neste contexto, o Método de Uzawa [40, 56, 58, 59] para desacoplar a pressão e a velocidade. Os sistemas lineares resultantes para a pressão e para a velocidade são resolvidos com o Método BiCG-Stab. 


\subsection{Discretização no espaço: malhas uniformes}

\subsubsection{Domínio euleriano}

O domínio computacional é um retângulo $\Omega=\left[A_{1}, B_{1}\right] \times\left[A_{2}, B_{2}\right]$, que inicialmente discretizaremos por uma malha uniforme cartesiana $\mathcal{G}_{\Omega}$, orientada na direção dos eixos coordenados. A malha $\mathcal{G}_{\Omega}$ está subdividida em $n_{x}$ células de espaçamento $\Delta x=\frac{A_{2}-A_{1}}{n_{x}}$ no eixo $x$ e $n_{y}$ células de espaçamento $\Delta y=\frac{B_{2}-B_{1}}{n_{y}}$ no eixo $y$. Denotamos o espaçamento da malha como $h$ quando $\Delta x=\Delta y$.

Entorno de todo o domínio, agregamos uma certa quantidade de células que terão valores dependentes das condições de contorno. Estas células são conhecidas como células fantasmas ou simplesmente fantasmas. A quantidade de células fantasmas na direção normal, $n_{g}$, é a mesma em cada uma das fronteiras ao redor do domínio $\Omega$. Os índices de uma célula fantasma $(i, j)$ satisfazem $\left(-n_{g}+1\right)<i<1$ ou $n_{x}<i<\left(n_{x}+n_{g}\right)$ e $\left(-n_{g}+1\right)<j<1$ ou $n_{y}<j<\left(n_{y}+n_{g}\right)$.

As posições das variáveis em cada célula do domínio computacional serão como as em "malhas deslocadas" (staggered grid), como as do tipo MAC (Marker-And-cell [50, 67]). Nestas malhas, as variáveis escalares são definidas no centro de cada célula e as vetoriais no meio das arestas da célula. As variáveis escalares são a pressão e as propriedades materiais do fluido, como a massa específica e a viscosidade. As variáveis vetoriais como a velocidade, a força dada pela interface da fronteira imersa ou a força viscoelástica, têm a primeira componente definida no meio das arestas verticais e a segunda componente no meio das arestas horizontais.

A principal razão dessa escolha está no fato de que com ela é possível definir convenientemente aproximações de segunda ordem para os operadores divergente e gradiente [85]. A Figura 3.1 mostra a localização das variáveis em uma célula $(i, j)$, com $1<i<n_{x}$ e $1<j<n_{y}$, da malha cartesiana discreta $\mathcal{G}_{\Omega}$. Definimos as componentes do tensor viscoelástico $\boldsymbol{S}$ (2.23) no centro das células [103], como mostra a Figura 3.1. Comentários sobre o posicionamento em cada célula de cada uma das componentes do tensor viscoelástico estão na Seção 3.4. As discretizações, tanto do modelo transiente como do modelo estacionário, foram realizadas em malhas deslocadas.

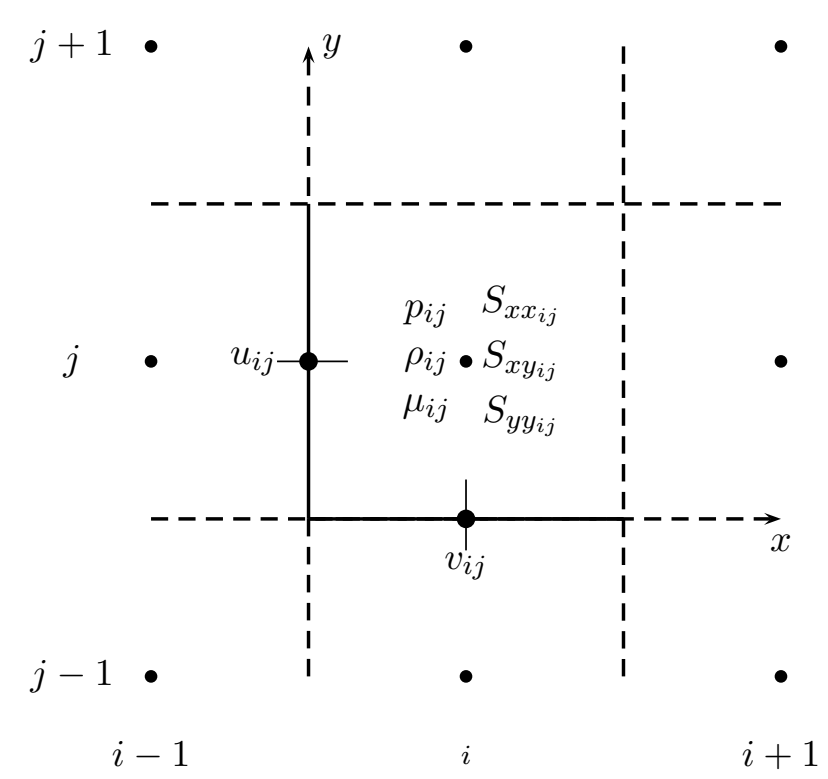

Figura 3.1: Localização das variáveis em uma célula computacional $(i, j)$ de $\mathcal{G}_{\Omega}$. 
Consideramos aqui que as variáveis definidas em uma célula $(i, j)$ estão ou no seu centro, ou em sua aresta oeste ou em sua aresta sul. Na literatura, é comum que uma variável localizada no centro da célula seja diferenciada de uma localizada no meio de uma aresta, agregando ao subíndice $i$ (similarmente ao $j$ ) da variável localizada no meio da aresta vertical (horizontal) o valor $\pm 1 / 2$. Neste trabalho, não acrescentamos nenhum valor aos índices das variáveis para identificar as respectivas posições delas nas células. Se a variável $\varphi$ pertence a célula $(i, j)$, esta variável é representada por $\varphi_{i j}$ independente da posição que tenha na célula. Para identificar a posição da variável $\varphi_{i j}$ na célula é preciso saber se a variável é escalar ou vetorial. No último caso, é necessário saber qual das duas componentes a variável representa. Só usamos algum subíndice com o valor $\pm 1 / 2$ quando é necessário explicar algum conceito onde é preciso entender claramente a posição da variável na célula. Nas discretizações, tivemos muito cuidado para que a posição das variáveis discretizadas sempre estivesse claramente identificada.

$\mathrm{Na}$ malha deslocada descrita anteriormente, introduzimos os operadores de diferenças finitas para aproximar os operadores diferenciais espacias. Para uma variável escalar $\varphi$ e uma variável vetorial $\boldsymbol{\psi}=\left(\psi_{1}, \psi_{2}\right)$, localizadas em suas posições correspondentes mostradas na Figura 3.1, definimos os seguintes operadores:

- Gradiente (G)

$$
(\mathbf{G} \varphi)_{i j}=\left(\left(\mathbf{D}_{\mathbf{1}} \varphi\right)_{i j},\left(\mathbf{D}_{\mathbf{2}} \varphi\right)_{i j}\right)
$$

onde $\mathbf{D}_{\mathbf{1}} \varphi=\frac{\partial \varphi}{\partial x}, \mathbf{D}_{\mathbf{2}} \varphi=\frac{\partial \varphi}{\partial y}$

$$
\begin{aligned}
& \left(\mathbf{D}_{1} \varphi\right)_{i j}=\frac{\varphi_{i j}-\varphi_{i-1 j}}{\Delta x} \\
& \left(\mathbf{D}_{2} \varphi\right)_{i j}=\frac{\varphi_{i j}-\varphi_{i j-1}}{\Delta y}
\end{aligned}
$$

- Divergente (D·)

$$
(\mathbf{D} \cdot \boldsymbol{\psi})_{i j}=\frac{\psi_{1, i+1 j}-\psi_{1, i j}}{\Delta x}+\frac{\psi_{2, i j+1}-\psi_{2, i j}}{\Delta y}
$$

- Laplaciano (L)

$$
\begin{aligned}
(\mathbf{L} \phi)_{i j} & =(\boldsymbol{D} \cdot \boldsymbol{G} \phi)_{i j} \\
& =\frac{\phi_{i+1 j}-2 \phi_{i j}+\phi_{i-1 j}}{\Delta x^{2}}+\frac{\phi_{i j+1}-2 \phi_{i j}+\phi_{i j-1}}{\Delta y^{2}}, \\
(\mathbf{L} \psi)_{i j} & =\left(\left(\mathbf{L} \psi_{1}\right)_{i j},\left(\mathbf{L} \psi_{2}\right)_{i j}\right),
\end{aligned}
$$

onde, para $k=1,2$,

$$
\left(\mathbf{L} \psi_{k}\right)_{i j}=\frac{\psi_{k, i+1 j}-2 \psi_{k, i j}+\psi_{k, i-1 j}}{\Delta x^{2}}+\frac{\psi_{k, i j+1}-2 \psi_{k, i j}+\psi_{k, i j-1}}{\Delta y^{2}} .
$$




\subsubsection{Domínio lagrangiano}

Como vimos anteriormente, a discretização das equações transientes de Navier-Stokes (ou das equações estacionárias de Stokes) é feita em uma malha euleriana. A discretização das equações para a interface de separação, dadas pelo Método da Fronteira Imersa, é feita em uma malha lagrangiana. Com esta abordagem, as duas malhas são tratadas separadamente e as informações que são comuns às duas discretizações são atualizadas a cada passo no tempo por meio da discretização das equações de interpolação (2.10) e espalhamento (2.9). A Figura 3.2 mostra a discretização dos domínios lagrangiano e euleriano.

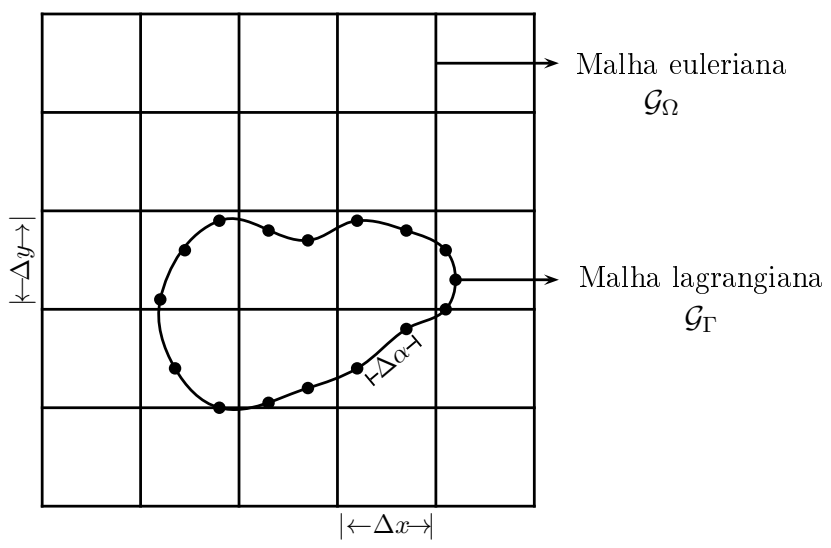

Figura 3.2: Malhas euleriana e lagrangiana.

A malha lagrangiana $\mathcal{G}_{\Gamma}$ é formada por uma coleção $n_{\alpha}$ de pontos igualmente espaçados. O espaçamento $\Delta \alpha$ entre dois pontos consecutivos $\mathbf{X}_{k}$ e $\mathbf{X}_{k+1}$ é, de certa forma, dependente da malha euleriana $[63,111]$. No presente trabalho, adota-se uma densidade de dois pontos lagrangianos por célula da malha euleriana [111], dada por

$$
\begin{gathered}
\Delta \alpha=\min \left(\frac{\Delta x}{2}, \frac{\Delta y}{2}\right), \\
n_{\alpha}=\frac{L_{\Gamma}}{\Delta \alpha},
\end{gathered}
$$

onde $L_{\Gamma}$ é o perímetro da interface. Na malha lagrangiana $\mathcal{G}_{\Gamma}$ os pontos são dados de forma consecutiva, $\mathbf{X}_{1}, \cdots, \mathbf{X}_{k-1}, \mathbf{X}_{k}, \mathbf{X}_{k+1}, \cdots, \mathbf{X}_{n_{\alpha}}$, onde $\mathbf{X}_{k}=\mathbf{X}\left(\alpha_{k}\right)=\left(X_{k}, Y_{k}\right)$ para $k=1, \cdots, n_{\alpha}$. A localização espacial dos pontos lagrangianos é arbitrária, uma vez que a interface de separação pode se mover por todo o domínio físico $\mathcal{G}_{\Omega}$. É possível, entretanto, determinar a posição relativa de um ponto de $\mathcal{G}_{\Gamma}$ na malha euleriana. A célula da malha euleriana que contém o ponto lagrangiano $\left(X_{k}, Y_{k}\right)$ é

$$
i=\left\lfloor\frac{X_{k}-A_{1}}{\Delta x}\right\rfloor+1 \text { e } j=\left\lfloor\frac{Y_{k}-B_{1}}{\Delta y}\right\rfloor+1
$$

onde $\lfloor\cdot\rfloor$ é a parte inteira de um número real.

É um fato conhecido da comunidade científica que trabalha com métodos de acompanhamento de fronteira que os pontos lagrangianos tendem a se aglomerar ou a se distanciar em regiões com alta curvatura ou com baixa curvatura, respectivamente. Para mantermos uma boa distribuição do espaçamento entre pontos lagrangianos consecutivos (sua "equidistribuição"), devemos empregar uma estratégia que mantenha esta propriedade e que a distância entre pontos seja mantida adequada. Ou seja, $\Delta \alpha>\min (\Delta x, \Delta y)$ ou $\Delta \alpha<\min \left(\frac{\Delta x}{4}, \frac{\Delta y}{4}\right)$. Quando o espaçamento entre pontos 
é inadequado, a quantidade de pontos lagrangianos deve ser dobrada se $\Delta \alpha>\min (\Delta x, \Delta y)$, ou reduzida pela metade se $\Delta \alpha<\min \left(\frac{\Delta x}{4}, \frac{\Delta y}{4}\right)$.

Na literatura, encontramos trabalhos $[62,113]$ que utilizam técnicas de equidistribuição baseadas em splines. A idea básica do algoritmo é representar a interface com um spline, calcular a sua integral de linha para obter o comprimento dessa curva e subdividir esse comprimento em $n_{\alpha}$ partes de comprimento igual.

No presente trabalho, empregamos outra estratégia de equidistribuição de pontos. Adicionamos uma velocidade tangencial auxiliar que mantêm os pontos lagrangianos em $\mathcal{G}_{\Gamma}$ igualmente espaçados ao longo de toda a interface. O procedimento para determinar esta velocidade auxiliar é dado em [30, 32].

O acoplamento entre os domínios lagrangiano e euleriano é dado pelos operadores de espalhamento (2.9) e interpolação (2.10). As formas discretas que envolvem o processo de espalhamento da densidade de força interfacial lagrangiana para a malha euleriana e o processo de interpolação das velocidades da malha euleriana para a malha lagrangiana são descritos na Seção 3.3.

\subsubsection{Função indicadora de fluido}

Determinar corretamente cada uma das propriedades físicas das diferentes fases envolvidas em um escoamento multifásico é um problema importante na área de simulação numérica. Os métodos numéricos para calcular de forma correta o escoamento de fluidos multifásicos, podem ser divididos em duas classes principais: métodos de captura de interfaces (front-capturing methods) e métodos de acompanhamento de interfaces (front-tracking methods). Nos métodos de captura de interfaces, tais como o método level set [73, 74, 92] e o de volume de fluido (VoF [84]), a interface do fluido está definida implicitamente por meio de uma função escalar (e.g. uma função distância ou uma fração de volume) que atua como uma função indicadora de fluido. Estes métodos capturam o movimento da interface sobre uma malha euleriana e refletem automaticamente as mudanças na topologia da interface. Por outro lado, os métodos de acompanhamento de interface [31, 78, 81, 106, 107] usam uma malha separada para seguir explicitamente o movimento da interface e assim obtêm, em geral, uma representação exata das quantidades geométricas da interface.

No presente trabalho, o controle das propriedades de cada material ou fluido é feito por meio de uma função indicadora de fluidos $\phi[31,111]$. Esta função indicadora satisfaz a propriedade de ser positiva na fase contínua e negativa na fase dispersa, mencionada na Seção 2.1.

Há diferentes técnicas para definir uma função que identifique as fases contínua e dispersa, sendo uma das mais conhecidas dada pela solução de uma equação de Poisson para $\phi$ [92, 107]. Uma outra proposta, robusta e que garante precisão para as regiões com elevadas curvaturas, para definir uma função indicadora de forma eficiente foi proposta por Ceniceros e Roma [31]. Esta abordagem emprega ideias de Geometria Computacional para a construção da função indicadora e tem baixo custo computacional, uma vez que não existe a necessidade de resolver sistemas lineares.

A ideia para a construção geométrica da função indicadora se baseia na função distância euclidiana. Dados os pontos lagrangianos, uma aproximação para a interface $\Gamma$ é representada pela união por segmentos de reta de cada par de pontos consecutivos, formando um polígono $P$. Cada um destes pontos são os vértices $V_{i}$ do polígono, como mostra a Figura 3.3.

Dessa maneira, definimos $\phi$ como a função indicadora somente em $T_{\Gamma}$, a qual é uma banda estreita centrada em $\Gamma$ de largura $2 \epsilon>0$. Fora dessa banda, $\phi$ é continuamente definida para que 

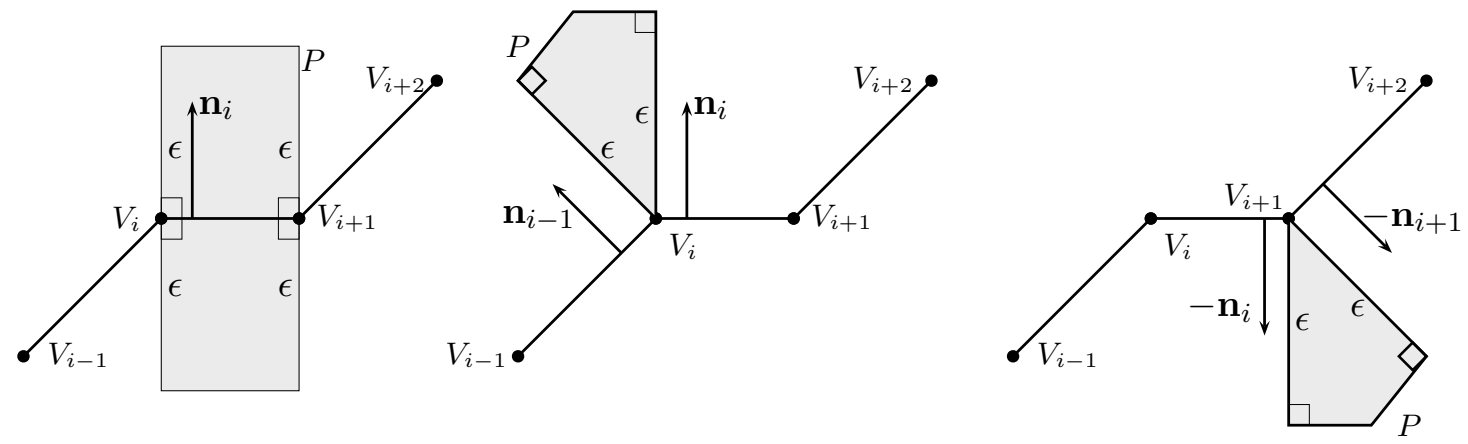

Figura 3.3: Conjunto de pontos dentro de uma distância $\epsilon$ a $\Gamma$, para o qual o ponto mais próximo está definido em bordas ou vértices.

tenha os valores $\pm \epsilon$, tal que

$$
\phi(\mathbf{x})= \begin{cases}-\epsilon, & \text { se } d(\mathbf{x})<-\epsilon \\ d(\mathbf{x}), & \text { se }-\epsilon \leq d(\mathbf{x}) \leq \epsilon \\ +\epsilon, & \text { se } d(\mathbf{x})>\epsilon\end{cases}
$$

onde $d(\mathbf{x})$ é a distância euclidiana de um dado ponto $\mathbf{x}$ até a interface $\Gamma$, para a qual o sinal é escolhido de acordo com a direção do vetor normal às arestas de $P$. A Figura 3.4 (a) mostra a função indicadora e a região $T_{\Gamma}$ (a banda) de tamanho $2 \epsilon$.

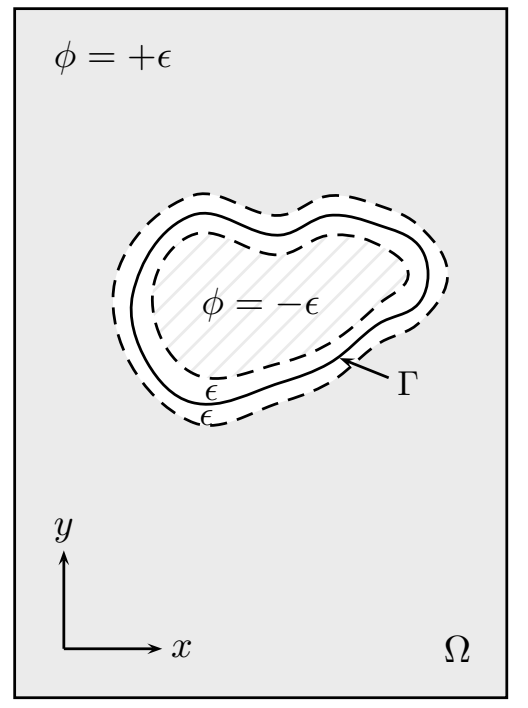

(a)

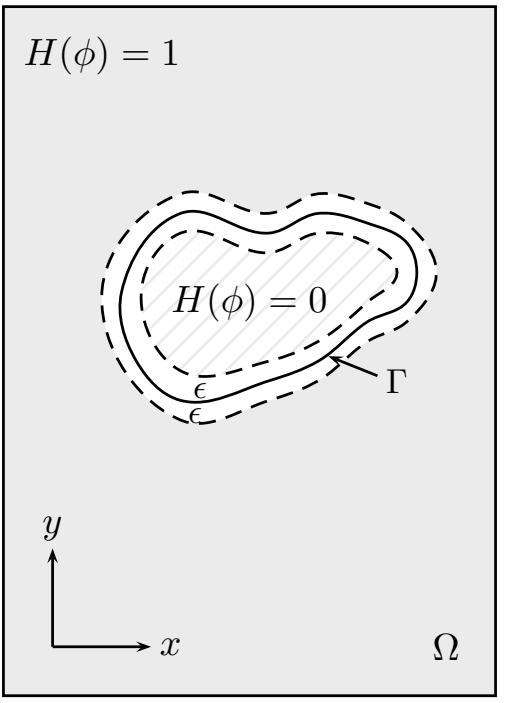

(b)

Figura 3.4: (a) Função indicadora discreta. (b) Função de Heaviside discreta.

Para determinar a função indicadora ao longo do tempo, é usado um algoritmo Closest Point Transform (CPT [66]). O algoritmo determina a menor distância euclidiana dos pontos da malha euleriana em relação à interface $\Gamma$. $\mathrm{O}$ algoritmo $\mathrm{CPT}$ é usado para encontrar o ponto mais próximo de $\Gamma$ e determinar a distância Euclidiana de todos os pontos da malha euleriana que se encontram em uma dada faixa $T_{\Gamma}$. Duas faixas são então definidas: uma para a região de "vértices" e outra para as "arestas" que representam a interface, como mostra a Figura 3.3. Este processo é executado a cada passo do tempo, após a posição da interface ter sido atualizada. Maiores detalhes sobre o algoritmo podem ser encontrados em [31]. 
A implementação do algoritmo da função indicadora no $A M R \mathscr{Q D}$ segue esta estratégia e foi desenvolvido por Villar [111]. Há também uma implementação, disponível na linguagem C, para a função indicadora desenvolvida por Macedo [37].

Empregamos a função indicadora de fluido em conjunto com a Função de Heaviside de forma que (mais detalhes na Seção 3.1.3)

$$
H_{\epsilon}(\phi)= \begin{cases}0 & \text { se } \phi<-\epsilon \\ \frac{1}{2}+\frac{\phi}{2 \epsilon}+\frac{1}{2 \pi} \sin \left(\frac{\pi \phi}{\epsilon}\right) & \text { se }-\epsilon \leq \phi \leq \epsilon \\ 1 & \text { se } \phi>\epsilon\end{cases}
$$

onde $\epsilon$ é a largura da faixa na função indicadora de fluidos (3.11). A Figura 3.4 (b) mostra a função Heaviside discreta. Observe que a discretização (3.12) tem uma variação suave entre 0 e 1 se $|\phi| \leq \epsilon$.

\subsection{Escoamentos incompressíveis transientes}

Nesta Seção, descrevemos a metodologia numérica para a solução de um escoamento incompressível transiente, modelado pelas equações de Navier-Stokes (2.7)-(2.8). A discretização das equações de Navier-Stokes é feita em uma malha euleriana usando o Método da Projeção de Chorin-Temam [34, 97], descrito na Seção 3.2.1. Na discretização no tempo, empregamos os métodos IMEX [5, 112], descritos na Seção 3.2.2, os quais evitam restrições temporais severas oriundas do termo difusivo, tal como é feito em [25, 32].

O modelo matemático bifásico e a seleção das fases é feita com o uso da função indicadora de fluidos (3.11) e a Função de Heaviside (3.12) [31, 73, 92, 106, 107]. A discretização das equações é a mesma sem distinguir a fase contínua da dispersa. Esta seleção automática é válida para as propriedades escalares (massa específica, viscosidade) e também para distinguir a fase newtoniana da não newtoniana, seguindo a formulação do Capítulo 2, nas equações (2.5), (2.6) e (2.18). Quando há uma interface de separação entre fases (fronteira imersa), temos a força interfacial que é calculada sobre a interface e é distribuída para a malha euleriana [48, 81, 85, 99].

\subsubsection{Método da Projeção de Chorin-Temam}

O objetivo do Método da Projeção consiste em desacoplar, na equação da conservação da quantidade de movimento (2.7), o cálculo da velocidade do da pressão. Chorin [34] e Temam [97], de maneira independente, constataram que para escoamentos incompressíveis a pressão não possui significado termodinâmico, forçando o campo de velocidade a satisfazer a condição de incompressibilidade (2.3). O Método da Projeção é um método do tipo "passo fracionado" [45], já que obtém um campo de velocidade auxiliar e com este se corrige a pressão [14, 34, 55, 85].

No Método da Projeção de Chorin-Temam para escoamentos incompressíveis, a velocidade e a pressão são determinadas em dois passos. No primeiro, um campo de velocidades auxiliar $\widetilde{\mathbf{u}}$ é calculado com a equação (2.7) da conservação da quantidade de movimento, desprezando a condição de incompressibilidade dada na equação (2.3). No segundo passo, o campo de velocidade auxiliar $\widetilde{\mathbf{u}}$ é projetado no espaço dos campos vetoriais com divergente nulo para calcular a correção da pressão. A partir dela corrigimos a pressão e o campo de velocidade u, satisfazendo a condição 
de incompressibilidade. O Método da Projeção tem como base o Teorema da Decomposição de Helmholtz-Hodge [35], cujo enunciado é apresentado a seguir.

Teorema 3.2.1. Seja $\Omega$ uma região no espaço (ou no plano) com fronteira $\partial \Omega$ suave. Um campo vetorial $\mathbf{w}$ em $\Omega$ pode ser decomposto de maneira única como

$$
\mathbf{w}=\mathbf{u}_{d}+\nabla \phi
$$

onde $\nabla \cdot \mathbf{u}_{d}=0$, $\phi$ é uma propriedade escalar e $\mathbf{u}_{d}$ é paralelo a $\partial \Omega$, isto é, $\mathbf{u}_{d} \cdot \mathbf{n}=0$ e $\mathbf{n}$ é o vetor normal a $\mathbf{u}_{\mathbf{d}}$.

A decomposição descrita na expressão (3.13) pode ser vista como a projeção do campo vetorial no espaço dos campos vetoriais com divergente nulo. Resumindo, para obtermos a solução das equações de Navier-Stokes usando o Método da Projeção executamos os seguintes passos:

Passo 1. Calcular o campo de velocidade auxiliar $\widetilde{\mathbf{u}}$

Passo 2. Projetar o campo de velocidade auxiliar $\widetilde{\mathbf{u}} \operatorname{com} \nabla \cdot \mathbf{u}=0$ e determinar a correção da pressão $\varphi$;

Passo 3. Corrigir o campo de velocidade u usando a nova correção da pressão $\varphi$;

Passo 4. Corrigir a pressão $p$.

Para solucionar o campo de velocidade auxiliar $\widetilde{\mathbf{u}}$ usaremos os esquemas IMEX definidos a seguir.

\subsubsection{Discretização no tempo: métodos implícitos-explícitos}

Para uma equação da forma

$$
u_{t}=g(u)+f(u)
$$

onde $g$ representa um termo difusivo e $f$ um termo advectivo, Ascher et al. [5] propõem uma discretização com esquemas temporais implícitos-explícitos (aos quais nós temos nos referido simplesmente por métodos "IMEX"). Tais métodos tratam explicitamente o termo advectivo e implicitamente o termo difusivo. O tratamento explícito do termo advectivo evita a resolução de um sistema não linear e o tratamento implícito do termo difusivo evita a escolha de um tamanho restritivo para o passo de tempo $\Delta t$.

Os métodos IMEX são obtidos por meio de expansões em séries de Taylor da equação (3.14) as quais, dependendo da ordem desejada para o método, determinam o valor de constantes apropriadas para a discretização. Inicialmente, as discretizações que podiam ser usadas com os métodos IMEX precisavam que o passo de tempo $\Delta t$ fosse constante em cada passo temporal. Esta restrição foi derrubada por Wang e Ruuth [112], que apresentaram uma forma geral de um método IMEX de $s$ passos, com um tamanho de passo $\Delta t$ variável. Os métodos de $s$ passos possuem ordem de aproximação $s$.

No presente trabalho, serão apresentados os esquemas IMEX de dois passos que aceitam o uso de $\Delta t$ variável ou constante. 
Consideremos três instantes de tempo $t^{n-1}, t^{n}$ e $t^{n+1}$, de forma que o tamanho do passo do tempo seja $\Delta t_{n}=t^{n}-t^{n-1}$ e $\Delta t_{n+1}=t^{n+1}-t^{n}$. A forma geral de uma aproximação para a equação (3.14) com o uso de esquemas IMEX de dois passos e ordem dois, e com tamanho de passo de integração variável, é dada pela expressão [112]

$$
\frac{\alpha_{2} u^{n+1}+\alpha_{1} u^{n}+\alpha_{0} u^{n-1}}{\Delta t_{n+1}}=\theta_{2} g^{n+1}+\theta_{1} g^{n}+\theta_{0} g^{n-1}+\beta_{1} f^{n}+\beta_{0} f^{n-1}
$$

onde $u^{k}, f^{k}$ e $g^{k}$ são uma aproximação para $u\left(t^{k}\right), f\left(u^{k}\right)$ e $g\left(u^{k}\right)$ respectivamente, sendo $k=$ $n-1, n, n+1$, e $\alpha_{i}, \beta_{i}$ e $\theta_{i}$ parâmetros que dependem de $\Delta t_{n+1}$ e $\Delta t_{n}$, com $\alpha_{2} \neq 0$ e $\theta_{2} \neq 0$. Os parâmetros $\alpha_{i}, \theta_{i}$ e $\beta_{i}$ são dados por:

$$
\begin{gathered}
\alpha_{2}=\frac{\Delta t_{n}+2 \gamma \Delta t_{n+1}}{\Delta t_{n}+\Delta t_{n+1}}, \alpha_{1}=\frac{(1-2 \gamma) \Delta t_{n+1}-\Delta t_{n}}{\Delta t_{n}}, \alpha_{0}=\frac{(2 \gamma-1) \Delta t_{n+1}^{2}}{\Delta t_{n}}\left(\Delta t_{n}+\Delta t_{n+1}\right) \\
\theta_{2}=\gamma+\frac{\Delta t_{n} c}{2 \Delta t_{n+1}}, \theta_{1}=1-\frac{\Delta t_{n} c}{2 \Delta t_{n+1}}-\frac{c}{2}-\gamma, \theta_{0}=\frac{c}{2} \\
\beta_{1}=\frac{\gamma \Delta t_{n+1}}{\Delta t_{n}}+1, \beta_{0}=-\frac{\gamma \Delta t_{n+1}}{\Delta t_{n}}
\end{gathered}
$$

A forma paramétrica de (3.14) em função dos coeficientes $\alpha_{i}, \theta_{i}$ e $\beta_{i}$ (3.16)-(3.18) permite que, ao combinarmos $\gamma$ e $c$, possamos obter diferentes métodos de aproximação temporal. Para os esquemas de dois passos é possível escolher dentre os métodos de Cranck-Nicolson-Adams-Bashforth (CNAB), Cranck-Nicolson-Adams-Bashforth Modificado (MCNAB), Gear extrapolado e Cranck-NicolsonLeap-Frog (CNLF). Os esquemas CNAB, MCNAB, Gear e CNLF são obtidos, respectivamente, ao se escolher o par de parâmetros $(c, \gamma)$ da forma, $\left(0, \frac{1}{2}\right),\left(\frac{1}{8}, \frac{1}{2}\right),(0,1)$ e $(1,0)$.

Os esquemas temporais IMEX de dois passos precisam de um esquema temporal de um passo para aproximar o primeiro passo de integração. Para a aproximação no primeiro passo no tempo, usamos o esquema IMEX Euler semi-implícito de um passo e de primeira ordem, dado pela expressão

$$
\frac{u^{n+1}-u^{n}}{\Delta t_{n+1}}=g(u)^{n+1}+f(u)^{n} .
$$

Para a solução da equação da conservação da quantidade de movimento (2.7), empregamos uma estratégia semi-implícita para tratar a não linearidade do termo difusivo quando a viscosidade $\mu$ é variável. Esta estratégia pode ser encontrada em [29, 39, 46] e consiste em somar e subtrair a (2.7) o termo viscoso $\lambda \nabla^{2} \mathbf{u}$. Empregando $\lambda=c_{1}|\mu|_{\text {max }}$, com $c_{1}>0,(2.7)$ pode ser reescrita como

$$
\rho \frac{\partial \mathbf{u}}{\partial t}=\lambda \nabla^{2} \mathbf{u}-\nabla p+\mathbf{f}(\mathbf{u})
$$

onde

$$
\mathbf{f}(\mathbf{u})=-\lambda \nabla^{2} \mathbf{u}-\rho(\mathbf{u} \cdot \nabla) \mathbf{u}+\nabla \cdot\left[\mu\left(\nabla \mathbf{u}+\nabla \mathbf{u}^{T}\right)\right]+\alpha_{p} \nabla \cdot \mathbf{S}(\mathbf{x})+\mathbf{f}_{\mathbf{s}}
$$




\subsubsection{Resumo da discretização do modelo transiente}

Na discretização para as equações de Navier-Stokes (2.7)-(2.8), para um escoamento incompressível de um fluido bifásico, usamos o Método da Projeção para desacoplar a velocidade e a pressão e a metodologia IMEX para a discretização temporal, apresentadas respectivamente nas seções 3.2.1 e 3.2.2.

A discretização temporal da equação da conservação da quantidade de movimento (2.7), com o método IMEX de segunda ordem, é dada por

$$
\rho^{n} \frac{\alpha_{2} \mathbf{u}^{n+1}+\alpha_{1} \mathbf{u}^{n}+\alpha_{0} \mathbf{u}^{n-1}}{\Delta t_{n+1}}=\theta_{2} \mathbf{g}^{n+1}+\theta_{1} \mathbf{g}^{n}+\theta_{0} \mathbf{g}^{n-1}+\beta_{1} \mathbf{f}^{n}+\beta_{0} \mathbf{f}^{n-1},
$$

onde $\mathbf{f}$ foi definida em (3.21) e

$$
\mathbf{g}(\mathbf{u})=\lambda \nabla^{2} \mathbf{u}-\nabla p
$$

Para $l=n-1, n, n+1$ e $k=n-1, n$, o esquema semi-implícito é dado por

$$
\begin{gathered}
\mathbf{g}^{l}=-\mathbf{G} p^{l}+\lambda \mathbf{L} \mathbf{u}^{l} \mathrm{e} \\
\mathbf{f}^{k}=-\lambda \mathbf{L} \mathbf{u}^{k}-\rho\left(\mathbf{u}^{k} \cdot \mathbf{G}\right) \mathbf{u}^{k}+\mathbf{D} \cdot\left[\mu\left(\mathbf{G} \mathbf{u}^{k}+\left(\mathbf{G} \mathbf{u}^{k}\right)^{T}\right)\right]+\alpha_{p} \mathbf{D} \cdot \mathbf{S}^{k}+\mathbf{f}_{s}^{k}
\end{gathered}
$$

Partindo desta discretização temporal, o acoplamento da pressão e da velocidade é tratado seguindo os passos descritos na seção 3.2.1, como segue

$$
\begin{gathered}
\frac{\alpha_{2} \widetilde{\mathbf{u}}+\alpha_{1} \mathbf{u}^{n}+\alpha_{0} \mathbf{u}^{n-1}}{\Delta t^{n+1}}=\theta_{2}\left(\frac{\lambda \mathbf{L} \widetilde{\mathbf{u}}-\mathbf{G} p^{n}}{\rho^{n}}\right)+\frac{\theta_{1} \mathbf{g}^{n}+\theta_{0} \mathbf{g}^{n-1}}{\rho^{n}}+\frac{\beta_{1} \mathbf{f}^{n}+\beta_{0} \mathbf{f}^{n-1}}{\rho^{n}}, \\
\widetilde{\mathbf{u}}=\mathbf{u}^{n+1}+\frac{\Delta t}{\alpha_{2}} \mathbf{G} \varphi \\
\mathbf{D} \cdot \mathbf{u}^{n+1}=0
\end{gathered}
$$

onde $\widetilde{\mathbf{u}}$ é uma velocidade auxiliar determinada da equação (3.26) e $\varphi$ é a correção da pressão obtida de (3.26)-(3.27).

Para que a condição de incompressibilidade (3.28) seja satisfeita, de (3.27) obtemos a equação elíptica para a correção da pressão

$$
\mathbf{D} \cdot\left[\frac{1}{\rho^{n}} \mathbf{G} \varphi\right]=\frac{\alpha_{2}}{\Delta t} \mathbf{D} \cdot \widetilde{\mathbf{u}}
$$

donde o campo de velocidade $\mathbf{u}^{n+1}$ e a pressão $p^{n+1}$ corregidos estão, respectivamente, dados por

$$
\begin{array}{r}
\mathbf{u}^{n+1}=\widetilde{\mathbf{u}}-\frac{\Delta t}{\alpha_{2}} \frac{\mathbf{G} \varphi}{\rho^{n}}, \\
p^{n+1}=p^{n}+\varphi .
\end{array}
$$

Uma observação importante sobre as condições de contorno numéricas para a velocidade e a pressão é que as fronteiras com condição de contorno de Dirichlet(Neumann) para a velocidade devem ter condição de contorno de Neumann(Dirichlet) para a pressão. Quando a condição de contorno para a velocidade é periódica em uma ou ambas as direções, a pressão também terá esta condição. 
Os sistemas oriundos das equações (3.26) e (3.29) são resolvidos usando o método multigrid (multinível-multigrid) geométrico com ciclo-V e com relaxador Gauss-Seidel preto-vermelho [65, 85, 102, 104, 111], da implementação AMR2D [110]. Por outro lado, será apresentado no Capítulo 4, uma nova ideia para ser empregada na solução destes sistemas, usando uma representação matricial [42, $80,82,88]$.

Nesta tese, a solução numérica das equações de Navier-Stokes foi tomado da implementação AMR2D [110, 111], para a qual foi incrementada a discretização temporal paramétrica IMEX e o tensor viscoelástico. Esta discretização pode ser estendida para o caso 3D da implementação AMR3D [71]. Mais detalhes sobre a implementação espacial e temporal para a solução das equações de Navier-Stokes e sobre o método multinível-multigrid podem ser encontradas em [71, 111].

Para completar a solução das equações de Navier-Stokes (2.7)-(2.8), nas seções 3.3 e 3.4 apresentamos a discretização numérica para a força interfacial e para o tensor viscoelástico, respectivamente.

\subsection{Discretização das equações lagrangianas}

Para que o acoplamento entre os domínios lagrangiano e euleriano seja possível, devemos obter uma forma discreta para os processos de espalhamento (2.9) e interpolação (2.10), definidos para o espalhamento da densidade de força interfacial lagrangiana para a malha euleriana e para a interpolação das velocidades da malha euleriana para a malha lagrangiana.

Dado que a Função Delta de Dirac tem papel de destaque nestes processos de espalhamento e de interpolação, da troca de informações entre as malhas euleriana e lagrangiana, iniciaremos por sua discretização.

A Função Delta de Dirac $\delta$ e a Função de Heaviside $H$ satisfazem que $\delta(\phi)=\nabla H(\phi)$ [73]. Assim no caso discreto, com $H_{\epsilon}$ dado por (3.12), temos que a Função Delta de Dirac discreta $\delta_{h}$ é aproximada por

$$
\delta_{h}(\xi)= \begin{cases}0, & \text { se } \xi<-h \\ \frac{1}{2 h}+\frac{1}{2 h} \cos \left(\frac{\pi \xi}{h}\right), & \text { se }|\xi| \leq h \\ 0, & \text { se } \xi>h\end{cases}
$$

onde $\xi \in \mathbb{R}$ e $h$ é um parâmetro numérico dependente dos espaçamentos $\Delta x$ e $\Delta y$ da malha euleriana.

Tal escolha de $\delta_{h}$ fornece boas propriedades de regularização numa vizinhança da interface de separação e é motivada por um conjunto de condições de compatibilidade descritas por Peskin [81]. Outras possíveis aproximações para esta função podem ser encontradas em [116].

Uma propriedade importante para as aproximações com a Função Delta de Dirac discreta no caso bidimensional [81] é que $\delta_{h}(\mathbf{x})=\delta_{h}(x) \delta_{h}(y)$, no caso em que $\mathbf{x}$ é um ponto no espaço bidimensional.

Sejam $\mathcal{S}_{n}$ e $\mathcal{S}_{n}^{*}$ os operadores discretos de espalhamento (2.9) e de interpolação (2.10), respectivamente, no instante de tempo $t^{n}$. Estes operadores são dados por

$$
\begin{gathered}
\left(\mathcal{S}_{n} G\right)(\mathbf{x})=\sum_{\alpha \in \mathcal{G}_{\Gamma}} G(\alpha) \delta_{h}\left(\mathbf{x}-\mathbf{X}^{n}(\alpha)\right) \Delta \alpha \\
\left(\mathcal{S}_{n}^{*} w\right)(\alpha)=\sum_{\mathbf{x} \in \mathcal{G}_{\Omega}} w(\mathbf{x}) \delta_{h}\left(\mathbf{x}-\mathbf{X}^{n}(\alpha)\right) \Delta x \Delta y
\end{gathered}
$$

onde $\mathbf{x} \in \mathcal{G}_{\Omega}, \mathbf{X}^{n}(\alpha)=\mathbf{X}\left(\alpha, t^{n}\right)$ e $G$ e $w$ são funções tais que $G(\alpha) \in \mathcal{G}_{\Gamma}$ e $w(\mathbf{x}) \in \mathcal{G}_{\Omega}$. 
Note que

$$
\begin{gathered}
\left(\mathcal{S}_{n} \mathbf{F}\right)\left(\mathbf{x}_{i j}\right)=\sum_{k=1}^{n_{\alpha}} \mathbf{F}^{n}\left(\alpha_{k}\right) \delta_{h}\left(\mathbf{x}_{i j}-\mathbf{X}_{k}^{n}\right) \Delta \alpha \\
\left(\mathcal{S}_{n}^{*} \mathbf{u}\right)\left(\alpha_{\mathbf{k}}\right)=\sum_{i, j} \mathbf{u}_{i j}^{n} \delta_{h}\left(\mathbf{x}_{i j}-\mathbf{X}_{k}^{n}\right) \Delta x \Delta y
\end{gathered}
$$

\subsubsection{Discretização para a equação de movimento da interface}

Como foi dito na Seção 3.1.2, os pontos lagrangianos precisam estar igualmente espaçados. No presente trabalho, para manter os pontos igualmente espaçados adicionamos às velocidades da interface uma velocidade tangencial $u_{a}(\mathbf{X}, t)[30,32]$, com

$$
u_{a}(\mathbf{X}(\alpha, t), t)=-u_{t}(\mathbf{X}, t)+\int_{0}^{\alpha}\left[\sigma_{\alpha} \kappa u_{n}-\left\langle\sigma_{\alpha} \kappa u_{n}\right\rangle\right] d \alpha^{\prime}
$$

onde $u_{t}=\mathbf{u} \cdot \mathbf{t}, u_{n}=\mathbf{u} \cdot \mathbf{n}, \sigma_{\alpha}=\sqrt{X^{2}(\alpha, t)+Y^{2}(\alpha, t)}$ é o comprimento métrico do arco, $\kappa$ é a curvatura média, $\mathbf{n}$ é o vetor normal unitário, $\mathbf{t}$ é o vetor tangente unitário e o operador \langle\rangle define uma média espacial.

Considerando que a equidistribuição dos pontos lagrangianos é feita juntamente com o cálculo do movimento da interface, adicionamos a velocidade auxiliar tangencial $u_{a}(\mathbf{X}, t) \boldsymbol{t}$, à velocidade da interface. Assim, a relação que interpola as velocidades eulerianas para os pontos lagrangianos (2.11) é agora dada por

$$
\mathbf{U}(\mathbf{X}(\alpha, t), t)=\int_{\Omega} \mathbf{u}(\mathbf{x}, t) \delta(\mathbf{x}-\mathbf{X}(\alpha, t)) d \mathbf{x}+u_{a}(X(\alpha, t), t) \mathbf{t} .
$$

A discretização para obter a nova posição dos pontos lagrangianos, dada pela condição de não deslizamento (2.10), é feita seguindo a ideia da discretização temporal paramétrica IMEX. Neste caso, não temos nenhum termo difusivo e $g=0$ em (3.14). Por tanto, a discretização obtida é explícita e é dada por

$$
\frac{\alpha_{2} \mathbf{X}^{n+1}+\alpha_{1} \mathbf{X}^{n}+\alpha_{0} \mathbf{X}^{n-1}}{\Delta t_{n+1}}=\beta_{1} \mathbf{U}^{n}+\beta_{0} \mathbf{U}^{n-1}
$$

onde $\alpha_{i}$ e $\beta_{j}$ para $i=0,2$ e $j=0,1$ estão definidos pelas expressões (3.16)-(3.18) e para $k=n-1, n$

$$
\mathbf{U}^{k}=\left(\mathcal{S}_{k}^{*} \mathbf{u}\right)(\alpha)+u_{a}^{k}\left(\mathbf{X}^{k}(\alpha), t\right) \mathbf{t}
$$

A aproximação de (3.39) com o Método de Gear extrapolado [32] é obtida quando o par de parâmetros $(c, \gamma)$ é $(0,1)$.

Com a posição dos pontos lagrangianos no passo de tempo $n+1$, podemos calcular a função indicadora $\phi^{n+1}$ e assim atualizar as propriedades que dependem dela, tais como a densidade e a viscosidade por meio de

$$
\begin{gathered}
\rho^{n+1}=\rho^{-}+\left(\rho^{+}-\rho^{-}\right) H_{\epsilon}\left(\phi^{n+1}\right), \\
\mu^{n+1}=\mu^{-}+\left(\mu^{+}-\mu^{-}\right) H_{\epsilon}\left(\phi^{n+1}\right) .
\end{gathered}
$$


A discretização da densidade de força lagrangiana via formulações clássica e híbrida, a discretização dos operadores de espalhamento (2.9) e de interpolação (2.10) e a equidistribuição de pontos foram previamente implementados e fazem parte do código AMR2D [110]. Utilizamos tal implementação para dar os primeiros passos na construção da metodologia adaptativa para escoamentos incompressíveis transientes bifásicos.

\subsubsection{Discretização da força lagrangiana}

A densidade de força $\mathbf{f}^{n+1}$ em (2.7), para tensão superficial $\sigma$ constante, pode ser obtida da formulação clássica (2.14) ou de uma formulação híbrida (2.16).

$\mathrm{Na}$ formulação clássica, a força é dada pelo espalhamento da densidade de força interfacial para o domínio euleriano por

$$
\mathbf{f}_{s}^{n}(\mathbf{x})=\left(\mathcal{S}_{n} \mathbf{F}\right)(\mathbf{x})
$$

onde $\mathbf{F}$ é dado por (2.13) e, no que segue, mostramos sua forma discreta.

Seja $D_{\alpha} \boldsymbol{\psi}=\left(D_{\alpha} \psi_{1}, D_{\alpha} \psi_{2}\right)=\left(\frac{\partial \psi_{1}}{\partial \alpha}, \frac{\partial \psi_{2}}{\partial \alpha}\right)$, para uma variável vetorial $\boldsymbol{\psi}=\left(\psi_{1}, \psi_{2}\right)$. Empregando diferenças centradas, a discretização deste operador para $i=1,2$ e $k \in \mathcal{D}_{\Gamma}$, é dada por

$$
\left(D_{\alpha} \psi_{i}\right)_{k}=\frac{\psi_{i, k+1}-\psi_{i, k-1}}{2 \Delta \alpha}
$$

Sabendo que a norma euclidiana de um vetor $\boldsymbol{\psi}$ é dada por $\|\boldsymbol{\psi}\|=\sqrt{\psi_{1}^{2}+\psi_{2}^{2}}$, podemos escrever a curvatura $(2.13)$ no $k$-ésimo ponto lagrangiano $\kappa_{k}$ como

$$
\begin{aligned}
\kappa_{k} & =\frac{\left\|D_{\alpha} \boldsymbol{t}_{k}\right\|}{\left\|D_{\alpha} \boldsymbol{X}_{k}\right\|} \\
& =\frac{\sqrt{\left(D_{\alpha} t_{x}\right)_{k}^{2}+\left(D_{\alpha} t_{y}\right)_{k}^{2}}}{\sqrt{\left(D_{\alpha} X\right)_{k}^{2}+\left(D_{\alpha} Y\right)_{k}^{2}}},
\end{aligned}
$$

onde $\mathbf{t}_{k}=\left(t_{x_{k}}, t_{y_{k}}\right)$ é o vetor tangente unitário no $k$-ésimo ponto lagrangiano dado por

$$
\mathbf{t}_{k}=\frac{D_{\alpha} \mathbf{X}_{k}}{\left\|D_{\alpha} \mathbf{X}_{k}\right\|}
$$

Por sua vez, o vetor normal unitário $\mathbf{n}_{k}$ é dado por

$$
\mathbf{n}_{k}=\frac{D_{\alpha} \mathbf{t}_{k}}{\left\|D_{\alpha} \mathbf{t}_{k}\right\|}
$$

Finalmente, de (3.45)-(3.47), concluímos que

$$
\begin{aligned}
\mathbf{F} & =\sigma \kappa_{k} \mathbf{n}_{k} \\
& =\sigma \frac{D_{\alpha} \mathbf{t}_{k}}{\sqrt{\left(D_{\alpha} X\right)_{k}^{2}+\left(D_{\alpha} Y\right)_{k}^{2}}}
\end{aligned}
$$

Detalhes sobre a discretização da formulação híbrida (2.16) podem ser encontrados em [92]. 


\subsection{Discretização do tensor viscoelástico}

Seja

$$
\mathbf{S}=\left[\begin{array}{ll}
\mathbf{S}_{x x} & \mathbf{S}_{x y} \\
\mathbf{S}_{x y} & \mathbf{S}_{y y}
\end{array}\right]
$$

a representação matricial para o tensor viscoelástico bidimensional $\mathbf{S}$. Como mencionamos na Seção 3.1.1, a definição da célula computacional que usamos para a discretização do tensor viscoelástico está dada na Figura 3.1. No presente trabalho, empregamos uma célula com as velocidades deslocadas nas faces oeste e sul para a primeira e segunda componente vetorial, respectivamente. As variáveis escalares estão definidas no centro da célula e cada uma das componentes do tensor viscoelástico está definida também no centro da célula. Essa definição de posicionamento dentro da célula segue a abordagem empregada por Castelo [103]. Outro posicionamento para o tensor na célula também é usado na literatura [99, 100]: a componente cruzada do tensor, $\mathbf{S}_{x y}$, é posicionada no canto inferior esquerdo da célula para que a discretização que aproxima o divergente do tensor $\nabla \cdot \mathbf{S}$ seja "mais natural" (recaia no centro da célula). Por outro lado, com a definição de célula que usamos, só é necessária uma aproximação usando uma média para determinar o valor desse divergente, o que é feito facilmente.

\subsubsection{Discretização do termo advectivo}

O Modelo Oldroyd-B para a aproximação do tensor viscoelástico pode ser visto como uma equação hiperbólica de adveç̧ão da forma

$$
\frac{\partial \mathbf{S}}{\partial t}+\mathbf{u} \cdot \nabla \mathbf{S}=\mathbf{f}
$$

onde $\mathbf{f}=\nabla \mathbf{u S}+\mathbf{S} \nabla \mathbf{u}^{T}-W_{i}^{-1}(\mathbf{S}-\mathbf{I})$.

A discretização para esta equação precisa ser consistente com a física do problema e, portanto, devemos ter cuidado com o Método de Diferenças Finitas em relação à direção da velocidade u. Em [96], encontramos um análise detalhada da solução numérica da equação (3.49) no contexto de diferenças finitas. A seguir, para facilitar a exposição da estratégia numérica empregada, consideraremos o caso particular em que (3.49) é escrita para uma função escalar $S$.

Para compreendermos melhor a natureza das dificuldades na resolução numérica de (3.49), consideremos em primeira instância o Método de Euler para a discretização no tempo. Para termos estabilidade numérica, o passo de integração temporal $\Delta t$ dependerá fortemente da escolha da discretização espacial. Sendo o esquema numérico consistente, pelo Teorema de Lax-Richtmyer, decorrerá, neste caso, a convergência para a solução exata de (3.49) no limite de $\Delta t$ e $\Delta x$ indo a zero [96].

Aplicando à equação (3.49) o Método de Euler, temos a discretização

$$
\frac{S^{n+1}-S^{n}}{\Delta t}+u \cdot \frac{\partial S^{n}}{\partial x}=f^{n}
$$

A aproximação do termo advectivo em (3.50) dependerá do sentido (do sinal) da velocidade do escoamento. Por exemplo, se usarmos diferenças adiantadas (3.51) teremos estabilidade numérica 
(e portanto convergência para a solução do problema) apenas se $u_{i}<0$ [96].

$$
\frac{S_{i}^{n+1}-S_{i}^{n}}{\Delta t}+u_{i} \cdot\left(\frac{S_{i+1}^{n}-S_{i}^{n}}{\Delta x}\right)=0 .
$$

Por outro lado, se usamos diferenças atrasadas

$$
\frac{S_{i}^{n+1}-S_{i}^{n}}{\Delta t}+u_{i} \cdot\left(\frac{S_{i}^{n}-S_{i-1}^{n}}{\Delta x}\right)=0
$$

só temos convergência quando $u_{i}>0$.

O processo de escolher uma aproximação para as derivadas parciais, dependendo do sinal da velocidade $u$ em cada ponto, é conhecido como diferenciação upwind ou upwinding.

Segundo o Teorema da Equivalência de Lax-Richtmyer [96], a convergência da aproximação de equações diferenciais lineares com esquemas de diferenças finitas é obtida se o esquema usado é consistente e estável.

A combinação do Método de Euler em (3.50) com o esquema upwind é consistente, mas pelo Teorema da Equivalência de Lax-Richtmyer deve ser estável para ser convergente. Para o método ser estável, este deve satisfazer a condição CFL (Courant-Friedreichs-Lewy Condition) dada por

$$
\Delta t<\frac{\Delta x}{\max |u|}
$$

Definindo $\alpha=\Delta t\left(\frac{\max |u|}{\Delta x}\right)$ temos estabilidade para $0<\alpha<1$. No caso de duas dimensões, a estabilidade é obtida para $0<\alpha<1 \mathrm{com}$

$$
\alpha=\Delta t\left(\frac{\max |\mathbf{u}|}{\min (\Delta x, \Delta y)}\right)
$$

Para conseguir uma ordem maior de convergência, poderíamos combinar o Método de Euler para a aproximação temporal com o esquema de diferenças finitas centradas de segunda ordem para a discretização espacial

$$
\frac{S_{i}^{n+1}-S_{i}^{n}}{\Delta t}+u_{i} \frac{S_{i+1}^{n}-S_{i-1}^{n}}{2 \Delta x}=0,
$$

mas para o método ser estável a condição de estabilidade é muito mais restritiva, pois $\Delta t$ deve ser da ordem de $\Delta x^{2}$ [96]. Para $\Delta t$ da ordem de $\Delta x$, o método não é estável [96].

Outra forma que pode ser usada para resolver a equação (3.49) é aproximar $S$ por uma função e depois calcular a derivada desta aproximação. Em cada ponto da discretização, aproximamos $S$ por uma função polinomial por partes com $r$ derivadas contínuas. Para isto, precisamos escolher $r+1$ pontos convenientes e tal escolha determinará a eficácia desta metodologia. O primeiro par de pontos são o ponto na posição para aproximar $x_{i}$ e o outro ponto é escolhido segundo a direção da velocidade como no upwinding, $x_{i-1}$ para $u_{i}>0$ e $x_{i+1}$ para $u_{i}<0$. Assim para aumentar a ordem de aproximação, adicionamos um novo ponto consecutivo à direita ou esquerda, controlando a derivada na ordem do ponto a adicionar.

Conforme Shu e Osher [73, 93], usamos para aproximar o polinômio a interpolação polinomial de diferenças divididas (polinômio interpolador na forma de Newton [23]).

O índice $i$ na célula indica a posição da variável que desejamos aproximar e $i+1 / 2$ a posição 
na metade entre a variável $i$ e a variável $i+1$. Por exemplo, a Figura 3.5 mostra uma aproximação nos nós da célula em uma dimensão em uma malha uniforme.

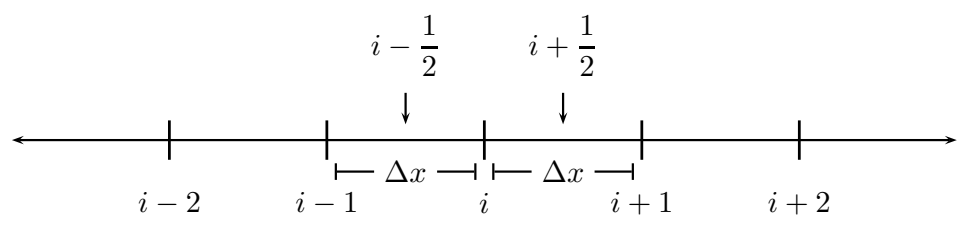

Figura 3.5: Distribuição de células em uma malha uniforme em uma direção.

Logo, a diferença dividida de ordem zero é $D_{i}^{0} S=q_{i}$ e a diferença dividida de primeira ordem é $D_{i+1 / 2}^{1}=\frac{D_{i+1}^{0} S-D_{i}^{0} S}{\Delta x}$, a qual está definida entre o nó $i$ e o nó $i+1$. Então, para obter a aproximação de terceira ordem, precisamos também da diferença de segunda ordem

$$
D_{i}^{2} S=\frac{D_{i+1 / 2}^{1} S-D_{i-1 / 2}^{1} S}{2 \Delta x}
$$

e da diferença dividida de terceira ordem

$$
D_{i+1 / 2}^{3} S=\frac{D_{i+1}^{2} S-D_{i}^{2} S}{3 \Delta x} .
$$

A construção polinomial de $S$ é da forma

$$
S(x)=q(x)=q_{0}(x)+q_{1}(x)+q_{2}(x)+q_{3}(x),
$$

onde $q_{r}$ é um polinômio de grau $r$ que depende da interpolação de $S$ por diferenças divididas.

Substituindo $x_{i}$ e derivando $S(x)$, obtemos a aproximação espacial desejada

$$
S^{\prime}\left(x_{i}\right)=\frac{\partial S_{i}}{\partial x}=q_{1}^{\prime}\left(x_{i}\right)+q_{2}^{\prime}\left(x_{i}\right)+q_{3}^{\prime}\left(x_{i}\right)
$$

Assim sendo, devemos determinar os outros pontos que podemos usar para determinar $q_{1}, q_{2}$ e $q_{3}$. O primeiro ponto $x_{k_{1}}$ é escolhido por meio de uma escolha upwind. Se $u_{i}>0$, escolhemos $k_{1}=i-1$ e, em caso contrário, escolhemos $k_{1}=i$. Decorre, portanto, que

$$
q_{1}(x)=\left(x-x_{i}\right) D_{k_{1}+1 / 2}^{1} S \quad \text { e } \quad q_{1}^{\prime}(x)=D_{k_{1}+1 / 2}^{1} S .
$$

Desta forma, temos que a aproximação de primeira ordem corresponde com a anteriormente mencionada pelo upwind em (3.50) e (3.51).

Escolheremos o próximo ponto de maneira a produzir a menor segunda derivada para a parábola a ser interpolada. Isto é, se $\left|D_{k_{1}}^{2} S\right| \leq\left|D_{k_{1}+1}^{2} S\right|$, escolhemos $k_{2}=k_{1}-1$ e caso contrário $k_{2}=k_{1}$. Portanto,

$$
q_{2}(x)=\left(x-x_{k_{1}}\right)\left(x-x_{k_{1}+1}\right) D_{k_{2}+1}^{2} S,
$$


com

$$
q_{2}^{\prime}\left(x_{i}\right)=\left(2 x_{i}-x_{k}-x_{k_{1}+1}\right) D_{k_{2}+1}^{2} S .
$$

Até aqui, com $s^{\prime}\left(x_{i}\right)=q_{1}^{\prime}\left(x_{i}\right)+q_{2}^{\prime}\left(x_{i}\right)$, temos uma aproximação para $s^{\prime}\left(x_{i}\right)$ de segunda ordem. Agora para obter a aproximação de terceira ordem, escolhemos o ponto que produza a menor terceira derivada no polinômio que passa pelos pontos correspondentes. Se $\left|D_{k_{2}+1 / 2}^{3} S\right| \leq\left|D_{k_{2}+3 / 2}^{3} S\right|$, então escolhemos $k_{3}=k_{2}$ e, em caso contrário, $k_{3}=k_{2}+1$, e assim

$$
q_{3}(x)=\left(x-x_{k_{2}}\right)\left(x-x_{k_{2}+1}\right)\left(x-x_{k_{2}+2}\right) D_{k_{3}+1 / 2}^{3} S,
$$

com

$$
q_{3}^{\prime}\left(x_{i}\right)=\left(3 x_{i}^{2}+x_{k_{2}+1} x_{k_{2}+2}+x_{k_{2}}\left(x_{k_{2}+1}+x_{k_{2}+2}\right)-2 x_{i}\left(x_{k_{2}}+x_{k_{2}+1}+x_{k_{2}+2}\right)\right) D_{k_{3}+1 / 2}^{3} S .
$$

Finalmente, definimos $q_{1}^{\prime}, q_{2}^{\prime}$ e $q_{3}^{\prime}$ para aproximar a derivada de $S$. Se desejarmos uma aproximação de ordem maior, devemos contínuar com este processo inserindo um a um os novos pontos. No caso bidimensional, este processo é feito para aproximar $\frac{\partial S}{\partial x}$ comparando com a velocidade na direção horizontal $u$ e para aproximar $\frac{\partial S}{\partial y}$ comparando com a velocidade na direção vertical $v$.

Para termos uma boa precisão tanto no tempo quanto no espaço, devemos escolher esquemas temporais de ordem mais alta para (2.42). O Método de Euler, usado no exemplo anterior, é apenas de primeira ordem. Shu e Osher [93] propuseram métodos de Runge-Kutta com a propriedade TVD (Total Variation Diminishing) para aumentar a ordem de aproximação na discretização temporal. TVD é uma propriedade de alguns esquemas de discretização para solucionar equações diferenciais hiperbólicas, definida por Harten [51]. Para entender o significado de um método TVD, vamos definir para (3.49) a variação total de $S$, como segue

$$
T V(S)=\int\left|\frac{\partial S}{\partial x}\right| d x
$$

No caso discreto, a variação total de $S$ é dada por

$$
T V(S)=\sum_{j}\left|S_{j+1}-S_{j}\right|
$$

Um método numérico que discretiza (3.49) é TVD se

$$
T V\left(S^{n+1}\right) \leq T V\left(S^{n}\right)
$$

Esta propriedade é importante, especialmente em aplicações com ondas de choque, porque garante que o esquema não está inserindo oscilações na aproximação obtida. Em nosso trabalho, usamos um Runge-Kutta TVD de segunda ordem, como aquele empregado por Ceniceros e Fisher [27, 51, 93] no contexto de fluidos viscoelásticos. Nós utilizamos na discretização temporal um método RungeKutta de segunda ordem (RK2 [24]) e para a aproximação espacial do termo advectivo dos modelos Oldroyd-B e FENE-P, usamos o esquema ENO de terceira ordem apresentado na próxima seção. 


\subsubsection{Modelo Oldroyd-B}

Ao usarmos para modelar o tensor $\mathbf{S}$ o Modelo Oldroyd-B (2.3.1)

$$
\mathbf{S}^{\nabla}=-W_{i}^{-1}(\mathbf{S}-I)
$$

obtivemos um modelo matemático que descreve a dinâmica de evolução do tensor viscoelástico cuja solução se reduz à resolução do sistema de equações diferenciais da forma

$$
\frac{\partial \mathbf{S}}{\partial t}=\mathbf{g}(\mathbf{S}, \mathbf{u})
$$

onde g está dado por (2.28) e (2.29).

Há uma grande variedade de métodos para obter a solução numérica de equações diferenciais desta forma [23, 24]. Usamos o método de Runge-Kutta de segunda ordem explícito [23, 24], que denotamos RK2, e obtivemos a discretização para o avanço no tempo $n+1$ para o tensor da seguinte forma

$$
\mathbf{S}^{n+1}=\frac{\mathbf{S}^{n}+\hat{\mathbf{S}}^{n+2}}{2}
$$

onde

$$
\begin{aligned}
& \hat{\mathbf{S}}^{n+1}=\mathbf{S}^{n}+\Delta t g\left(\mathbf{S}^{n}, u^{n+1}\right), \\
& \hat{\mathbf{S}}^{n+2}=\hat{\mathbf{S}}^{n+1}+\Delta t g\left(\hat{\mathbf{S}}^{n+1}, \mathbf{u}^{n+1}\right) .
\end{aligned}
$$

Na discretização de $\mathbf{g}\left(\hat{\mathbf{S}}^{n+1}, \mathbf{u}^{n+1}\right)$, devemos ter um cuidado especial com o termo advectivo. Inicialmente porque a discretização deve acompanhar a direção da velocidade e não gerar instabilidades numéricas por incompatibilidade com o problema físico. Depois, porque temos uma dependência numérica do número de Weissenberg [43, 54].

Se o método usado para discretizar o termo advectivo não é conveniente, quando aumentamos o número de Weissenberg a discretização pode não acompanhar o fenômeno e divergir [43, 54, 96]. Em 2005, Hulsen e colaboradores [54] estudaram a influência do número de Weissenberg ao discretizar a equação para o tensor viscoelástico com um esquema explícito de diferenças finitas de primeira ordem. Concluíram que além da condição de restrição temporal, há também uma restrição espacial que depende do número de Weissenberg, tornando de esse esquema demasiado restritivo. Uma solução apresentada para esta discretização baseia-se em uma mudança de variáveis [43, 54].

Por este motivo, usamos na discretização do termo advectivo o método ENO de terceira ordem explicado.

Dessa forma, temos que a discretização para o tensor na componente tensorial $\mathbf{S}_{x x}$ é dada por

$$
\underbrace{\frac{\partial S_{x x}}{\partial t}+}_{\text {RK2 }} \underbrace{u \frac{\partial S_{x x}}{\partial x}+v \frac{\partial S_{x x}}{\partial y}}_{\text {ENO }}=\cdots,
$$


e para os outros termos temos, em cada célula $(i, j)$, que

$$
\begin{aligned}
\left(S_{x x} \frac{\partial u}{\partial x}\right)_{i j} & =S_{x x, i j} \frac{u_{i+1 j}-u_{i j}}{\Delta x} \\
\left(S_{x x} \frac{\partial u}{\partial y}\right)_{i j} & =S_{x x, i j}\left(\frac{u_{i j+1}-u_{i j-1}}{2 \Delta y}+\frac{u_{i+1 j+1}-u_{i+1 j-1}}{2 \Delta y}\right) / 2 \\
& =S_{x y, i j} \frac{u_{i j+1}+u_{i+1 j+1}-u_{i j-1}-u_{i+1 j-1}}{4 \Delta y} .
\end{aligned}
$$

Assim, para esta componente do tensor temos, passo a passo, no RK2:

\section{Passo 1.}

$$
\begin{array}{r}
S_{x x_{1}}=\hat{S}^{n+1}=S_{x x}^{n}+\Delta t[\underbrace{-\left(u^{n+1} \frac{\partial S_{x x}^{n}}{\partial x}+v^{n+1} \frac{\partial S_{x x}^{n}}{\partial y}\right)_{1}}_{\text {ENO }}+2\left(S_{x x}^{n} \frac{\partial u^{n+1}}{\partial x}+S_{x y}^{n} \frac{\partial u^{n+1}}{\partial y}\right) \\
\left.-\omega i^{-1}\left(S_{x x}-1\right)+f_{x x}\right] ;
\end{array}
$$

\section{Passo 2.}

$$
\begin{array}{r}
S_{x x_{2}}=\hat{S}^{n+2} \\
=S_{x x_{1}}+\Delta t[\underbrace{-\left(u^{n+1} \frac{\partial S_{x x_{1}}}{\partial x}+v^{n+1} \frac{\partial S_{x x_{1}}}{\partial y}\right)_{1}}_{\text {ENO }}+2\left(S_{x x_{1}} \frac{\partial u^{n+1}}{\partial x}+S_{x y_{1}} \frac{\partial u^{n+1}}{\partial y}\right) \\
\left.-\omega i^{-1}\left(S_{x x_{1}}-1\right)\right]
\end{array}
$$

\section{Passo 3.}

$$
S_{x x}^{n+1}=\frac{\left(S_{x x}^{n}+S_{x x_{2}}\right)}{2} .
$$

De forma análoga, fazemos o mesmo processo para as outras duas componentes do tensor viscoelástico, determinando primeiro $S_{x x}^{n+1}$, depois $S_{x y}^{n+1}$ e por último $S_{y y}^{n+1}$. Estas discretizações são dependentes e portanto, em cada um dos passos, isso deve ser feito para cada uma das três componentes.

\subsubsection{Modelo FENE-P}

O Modelo FENE-P está dado por (2.31)-(2.32), onde $l$ é um parâmetro para o controle da extensibilidade média máxima das cadeias poliméricas e $\mathbf{C}$ é um tensor auxiliar intermediário empregado para determinar o tensor $\mathbf{S}[19,100,101]$.

Para obter da equação constitutiva para o tensor viscoelástico $\mathbf{S}$ usando o Modelo FENE-P, o tensor no instante de tempo $t=t^{n+1}$, devemos determinar primeiro o tensor $\mathbf{C}^{n+1}$ usando $\mathbf{C}^{n}, \mathbf{S}^{n}$ e $\mathbf{u}^{n+1}$ em (2.31) e depois calcular o tensor $\mathbf{S}^{n+1}$ em (2.32). 
Como vimos na Seção 2.3.2, (2.31) pode ser reescrita na forma

$$
\frac{\partial \mathbf{C}}{\partial t}=\mathbf{h}(\mathbf{C}, \mathbf{S}, \mathbf{u})
$$

onde $\mathbf{h}$ é dado pela expressão (2.39). Logo, a discretização temporal que usamos neste modelo é a mesma usada para discretizar o Modelo Oldroyd-B:

$$
\mathbf{C}^{n+1}=\frac{\mathbf{C}^{n}+\hat{\mathbf{C}}^{n+2}}{2}
$$

sendo

$$
\begin{aligned}
\hat{\mathbf{C}}^{n+1} & =\mathbf{C}^{n}+\Delta t \mathbf{h}\left(\mathbf{C}^{n}, \mathbf{S}^{n}, u^{n+1}\right), \\
\hat{\mathbf{C}}^{n+2} & =\hat{\mathbf{C}}^{n+1}+\Delta t \mathbf{h}\left(\hat{\mathbf{C}}^{n+1}, \hat{\mathbf{S}}^{n+1}, \mathbf{u}^{n+1}\right), \\
\hat{\mathbf{S}}^{n+1} & =\frac{\hat{\mathbf{C}}^{n+1}}{1-\left(\operatorname{tr}\left(\hat{\mathbf{C}}^{n+1}\right) / l^{2}\right)},
\end{aligned}
$$

onde, para $k=0,1$,

$$
\begin{aligned}
& \hat{\mathbf{C}}^{n+k+1}=\hat{\mathbf{C}}^{n+k}+\Delta t \mathbf{h}\left(\hat{\mathbf{C}}^{n+k}, \hat{\mathbf{S}}_{p}^{n+k}, \mathbf{u}^{n+1}\right) \\
& \mathbf{h}\left(\hat{\mathbf{C}}^{n+k}, \hat{\mathbf{S}}_{p}^{n+k}, \mathbf{u}^{n+1}\right)=-\mathbf{u}^{n+1} \cdot \nabla \hat{\mathbf{C}}^{n+k}+ \\
&+\left(\mathbf{G} \mathbf{u}^{n+1} \cdot \hat{\mathbf{C}}^{n+k}+\hat{\mathbf{C}}^{n+k} \cdot \mathbf{G u}^{T, n+1}\right)+ \\
&-W_{i}^{-1}\left(\hat{\mathbf{S}}_{p}^{n+k}-I\right), \\
& \hat{\mathbf{S}}^{n+k+1}=\frac{\hat{\mathbf{C}}^{n+k+1}}{1-\left(\operatorname{tr}\left(\hat{\mathbf{C}}^{n+k+1}\right) / l^{2}\right)} .
\end{aligned}
$$

Lembrando que $\operatorname{tr}\left(\hat{\mathbf{C}}^{n+1}\right)=\hat{\mathbf{C}}_{x x}^{n+1}+\hat{\mathbf{C}}_{y y}^{n+1}$.

Finalmente, a atualização para o tensor $\mathbf{S}^{n+1}$ é dada por

$$
\mathbf{S}^{n+1}=\frac{\mathbf{C}^{n+1}}{1-\left(\operatorname{tr}\left(\mathbf{C}^{n+1}\right) / l^{2}\right)}
$$

Lembramos que a condição inicial do tensor $\mathbf{C}$ é dada em (2.37).

\subsection{Escoamentos incompressíveis estacionários}

Resolveremos as equações estacionárias de Stokes (2.43) empregando o Método de Uzawa [4]. Esse método, no caso de escoamentos incompressíveis estacionários, tem o papel que o Método da Projeção tem no caso transiente: ele desacopla a pressão da velocidade [40, 56, 58, 59].

Assim, assumindo $\mu$ constante, obtivemos na Seção 2.3 as equações estacionárias de Stokes (2.44), onde $\mathbf{f}$ é a força total que atua no fluido. Estas equações junto à condição da continuidade (2.8), modelam o escoamento de um fluido estacionário incompressível.

Empregamos, para representar a forma discreta deste modelo, a seguinte notação para os ope- 
radores discretizados:

$$
\begin{array}{r}
-\mathbf{G} p+\mathbf{L u}=\mathbf{f}, \\
\mathbf{D} \cdot \mathbf{u}=0,
\end{array}
$$

onde $\mathbf{G}$ denota o operador gradiente, $\mathbf{L}$ o operador Laplaciano e $\mathbf{D}$ o divergente.

Para calcular a velocidade $\mathbf{u}$ e a pressão $p$, seguimos o Método de Uzawa [4, 36, 58, 69, 108], o qual por meio de aplicação de operadores e do uso da equação de continuidade, transforma as equações estacionárias de Stokes e a da continuidade, conseguindo isolar a pressão em uma única equação que não depende da velocidade. Este processo é descrito a seguir.

Inicialmente aplicamos $\mathbf{L}^{-1}$ em (3.62), obtendo assim

$$
-\mathbf{L}^{-1} \mathbf{G} p+\mathbf{u}=\mathbf{L}^{-1} \mathbf{f} .
$$

Aplicando a (3.64) D·, obtemos

$$
-\mathbf{D} \cdot \mathbf{L}^{-1} \mathbf{G} p+\mathbf{D u}=\mathbf{D L}^{-1} \mathbf{f} .
$$

Ao substituirmos (3.63) em (3.65), obtemos

$$
\mathbf{D} \cdot \mathbf{L}^{-1} \mathbf{G} p=-\mathbf{D L}^{-1} \mathbf{f}
$$

Podemos representar (3.66) como um sistema linear da forma

$$
\mathbf{A} p=\mathbf{g}
$$

onde $\mathbf{A}=\mathbf{D} \cdot \mathbf{L}^{-1} \mathbf{G}$ é um operador linear e $\mathbf{g}=-\mathbf{D} \cdot \mathbf{L}^{-1} \mathbf{f}$.

A relação para o campo de velocidade u, seguindo a discretização da pressão (3.67), é dada ao se isolar a velocidade $\mathbf{u}$ em (3.64)

$$
\begin{aligned}
\mathbf{u} & =\mathbf{L}^{-1} \mathbf{f}+\mathbf{L}^{-1} \mathbf{G} p \\
& =\mathbf{L}^{-1}(\mathbf{f}+\mathbf{G} p)
\end{aligned}
$$

Como discutiremos em maiores detalhes no Capítulo 4, para malhas bloco-estruturadas, o uso de refinamento adaptativo produzirá representações matriciais de $\mathbf{A}$, as quais, ao contrário do caso uniforme, não serão simétricas. Dado que nosso foco principal é a discretização neste contexto, para resolver o sistema linear resultante (3.67), devemos usar um método válido para matrizes que não sejam simétricas [117, 118]. Uma opção é o uso do Método do Gradiente Biconjugado Estabilizado (BiCG-Stab [13, 57, 108, 118]), o qual estendemos para o contexto de malhas bloco-estruturadas.

O Método BiCG-Stab é o resultado de uma combinação entre o Método do Gradiente Biconjugado (BiCG) e o Método dos Resíduos Mínimos Generalizados (GMRES) [13, 108]. O Método BiCG é uma generalização do Método do Gradiente Conjugado (CG) para matrizes não simétricas. Tais métodos pertencem à classe dos métodos iterativos e dependem do cálculo de produtos internos, vetores de resíduos e vetores de direção durante suas iterações. O Método BiCG-Stab, diferente do Método BiCG, não realiza operações como o cálculo da transposta de uma matriz. Maiores detalhes 
sobre estes métodos podem ser encontrados na literatura na área de Álgebra Linear Computacional.

O Algoritmo 1 descreve os passos para o calculo da pressão e da velocidade nas equações (3.62) e (3.63), seguindo o Método de Uzawa BiCG-Stab [57], para um valor inicial da pressão $\mathbf{p}^{(0)}$.

Algoritmo 1 (Uzawa BiCG-Stab).

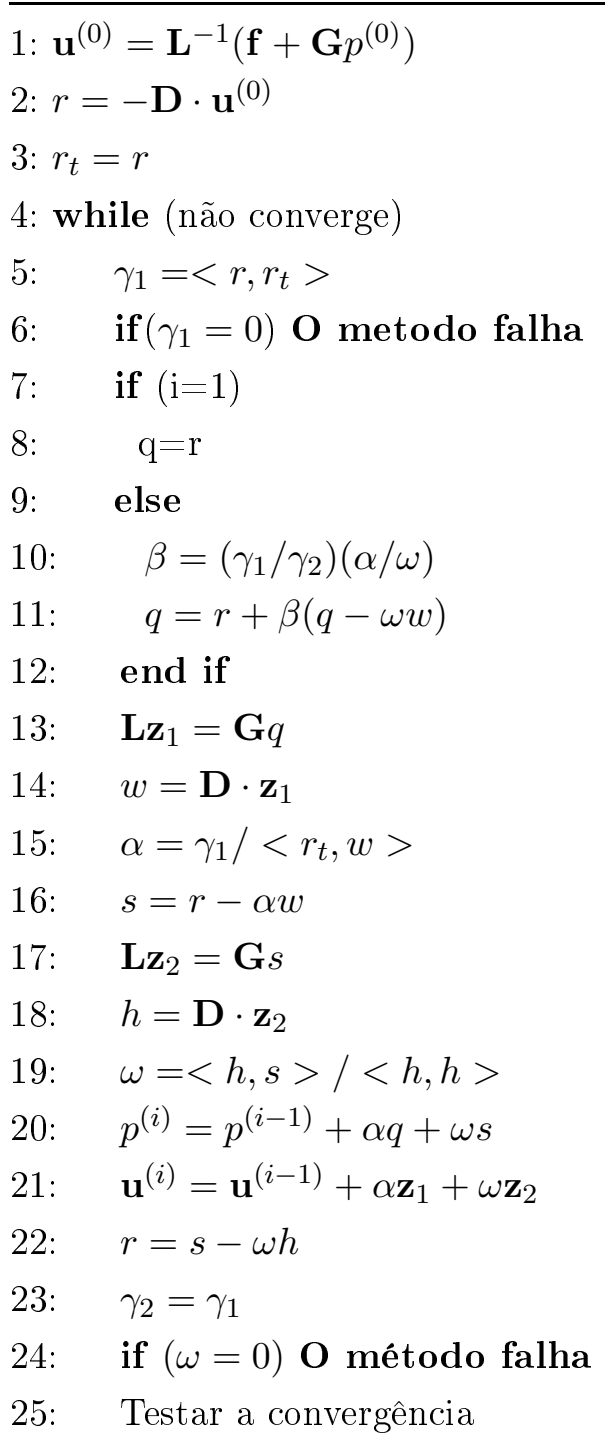

26: end while

No Algoritmo 1, o resíduo $r=\boldsymbol{D} \cdot \mathbf{u}$ vem da condição de incompressibilidade (2.8). Empregamos para ele a condição de contorno de Dirichlet homogênea, sem nos importarmos qual é a condição de contorno dada para a velocidade $\mathbf{u}$. Além disso, para as fronteiras do domínio nas quais a velocidade $\mathbf{u}$ tem condição de contorno de Dirichlet, os vetores de busca $\mathbf{z}_{1}$ e $\mathbf{z}_{2}$ terão condição de contorno de Dirichlet homogênea.

A velocidade e a pressão, no algoritmo apresentado, são calculadas em um mesmo laço de iteração. Isto porque (3.66) e (3.68) geram as mesmas direções de busca ao serem resolvidas simultaneamente com o Método BiCG-Stab.

Observemos que para cada passo de iteração do BiCG-Stab, devemos solucionar duas equações 
elípticas $L \mathbf{z}_{1}=G q$ e $L \mathbf{z}_{2}=G s$, onde $\mathbf{z}_{1}=\left(z_{1 u}, z_{1 v}\right)$ e $\mathbf{z}_{2}=\left(z_{2 u}, z_{2 v}\right)$ têm suas componentes definidas no meio da aresta na célula computacional, como mostra a Figura 3.1. Para solucionar essas equações, passamos a usar uma metodologia matricial que apresentaremos no Capítulo 4.

O uso de uma representação matricial para solucionar tais equações elípticas permite que exploremos o uso de bibliotecas numéricas gratuitas (com implementações variadas de diversos métodos, inclusive versões paralelas). No caso da discretização do domínio espacial em malha uniforme a matriz é simples e simétrica mas, quando usada malha bloco-estruturada com refinamento localizado, devemos ter muito cuidado para que a matriz consiga capturar os detalhes de interpolações na determinação dos valores nas células fantasmas.

\subsubsection{Comentários sobre a discretização da equação de Stokes em um fluido viscoelástico}

A discretização das equações estacionárias de Stokes para um fluido incompressível (3.62)-(3.63), com termo forçante $\mathbf{f}$, foi feita usando o Método de Uzawa. No caso de fluidos viscoelásticos com uma interface imersa, a força total é dada por $\mathbf{f}=-\alpha_{p} \mathbf{D} \cdot \mathbf{S}-\mathbf{f}_{s}$.

A discretização apresentada anteriormente, empregando o Método de Uzawa no contexto do Método BiCG-Stab, é feita supondo que $\mathbf{S}$ e $\mathbf{f}_{s}$ são conhecidos, sendo que as equações para a discretização temporal a serem resolvidas com estes métodos são

$$
\begin{gathered}
-\mathbf{G} p^{n+1}+\mathbf{L} \mathbf{u}^{n+1}=-\alpha_{p} \mathbf{D} \cdot \mathbf{S}^{n}-\mathbf{f}_{s}^{n} \\
\mathbf{D} \cdot \mathbf{u}^{n+1}=0 .
\end{gathered}
$$

A discretização espacial para a força viscoelástica, dado que cada uma das componentes do tensor são definidas no centro da célula e a força viscoelástica tem cada uma de suas componentes definida no ponto médio das respectivas arestas, como mostra a Figura 3.1, é dada por

$$
(\mathbf{D} \cdot \mathbf{S})_{i j}=\left[\begin{array}{l}
\left(\partial_{x} S_{x x}\right)_{i j}+\left(\partial_{y} S_{x y}\right)_{i j} \\
\left(\partial_{x} S_{x y}\right)_{i j}+\left(\partial_{y} S_{x y}\right)_{i j}
\end{array}\right] .
$$

Logo, para obter os valores discretos de cada uma das componentes de (3.71), temos

$$
\text { Para o centro de célula }\left\{\begin{array}{l}
\left(\partial_{x} S_{x x}\right)_{i j}^{c}=\frac{S_{x x, i+1 j}-S_{x x, i-1 j}}{2 \Delta x} \\
\left(\partial_{y} S_{x y}\right)_{i j}^{c}=\frac{S_{x y, i j+1}-S_{x y, i j-1}}{2 \Delta y} \\
\left(\partial_{x} S_{x y}\right)_{i j}^{c}=\frac{S_{x y, i+1 j}-S_{x y, i-1 j}}{2 \Delta x} \\
\left(\partial_{y} S_{y y}\right)_{i j}^{c}=\frac{S_{y y, i j+1}-S_{y y, i j-1}}{2 \Delta y}
\end{array} .\right.
$$

Dado que o divergente do tensor viscoelástico é definido no meio das arestas, temos que: 
Para a face $x\left\{\begin{array}{rl}\partial_{x} S_{x x}+\partial_{y} S_{x y} \\ \left(\partial_{x} S_{x x}\right)_{i j}=\frac{S_{x x_{i j}}-S_{x x_{i-1 j}}}{\Delta x} \\ \left(\partial_{y} S_{x y}\right)_{i j}=\left(\frac{S_{x y_{i j+1}}-S_{x y_{i j-1}}}{2 \Delta y}+\frac{S_{x y_{i-1 j+1}}-S_{x y_{i-1 j-1}}}{2 \Delta y}\right) / 2 \\ =\frac{S_{x y_{i j+1}}+S_{x y_{i-1 j+1}}-S_{x y_{i j-1}}-S_{x y_{i-1 j-1}}}{4 \Delta y}\end{array} ;\right.$

Para a face $y\left\{\begin{aligned}\left(\partial_{x} S_{x y}+\partial_{y} S_{y y}\right. & \\ & =\frac{S_{x y_{i+1 j}}+S_{x y_{i+1 j-1}}-S_{x y_{i-1 j}}-S_{x y_{i-1 j-1}}}{4 \Delta x} \\ & =\left(\frac{S_{x y_{i+1 j}}-S_{x y_{i-1 j}}}{2 \Delta x}+\frac{S_{x y_{i+1 j-1}}-S_{x y_{i-1 j-1}}}{2 \Delta x}\right) / 2 \\ \left(\partial_{y} S_{y y}\right)_{i j} & =\frac{S_{y y_{i j}}-S_{y y_{i j-1}}}{\Delta y}\end{aligned}\right.$

Os passos para a discretização das equações estacionárias de Stokes contendo ao menos uma fase viscoelástica, dados $\mathbf{u}^{n}, \mathbf{S}^{n}$ e $\mathbf{f}_{s}^{n}$, se escrevem como:

Passo 1. Defina $\mathbf{f}=\mathbf{f}_{s}^{n}+\beta \nabla \cdot \mathbf{S}^{n}$

Passo 2. Use o Método de Uzawa para encontrar $p^{n+1}$ e $\mathbf{u}^{n+1}$;

Passo 3. Determine $\mathbf{S}^{n+1}$ com um dos modelos Oldroyd-B ou FENE-P, usando RK2

$$
\mathbf{S}^{n+1}=\frac{\mathbf{S}^{n}+\hat{\mathbf{S}}^{n+2}}{2}
$$

Passo 4. Espalhe a força e movimentar e atualizar a posição dos pontos lagrangianos.

\subsection{Sumário da metodologia numérica}

Terminamos esse Capítulo com um sumário da discretização para um passo no tempo típico. Por simplicidade, não empregamos subíndices espaciais $i, j$. Dos valores calculados da pressão $p^{k}$, a posição dos pontos na interface $\mathbf{X}^{k}$, o campo de velocidade $\mathbf{u}^{k}$, a densidade de força da interface $\mathbf{f}_{s}^{k}$ e do tensor viscoelástico $\mathbf{S}^{k}$, no tempo $t=t^{k}$ para $k=n, n-1$, atualizamos o valor de cada uma destas variáveis, no instante de tempo $t^{n+1}$, para os diferentes modelos resumidos na Secção 2.4, como segue. 


\subsubsection{Escoamentos incompressíveis transientes de fluidos bifásicos}

$$
\begin{gathered}
\frac{\alpha_{2} \widetilde{\mathbf{u}}+\alpha_{1} \mathbf{u}^{n}+\alpha_{0} \mathbf{u}^{n-1}}{\Delta t^{n+1}}=\theta_{2}\left(\frac{\lambda \mathbf{L} \widetilde{\mathbf{u}}-\mathbf{G} p^{n}}{\rho^{n}}\right)+\frac{\theta_{1} \mathbf{g}^{n}+\theta_{0} \mathbf{g}^{n-1}}{\rho^{n}}+\frac{\beta_{1} \mathbf{f}^{n}+\beta_{0} \mathbf{f}^{n-1}}{\rho^{n}}, \\
\mathbf{D} \cdot\left[\frac{1}{\rho^{n}} \mathbf{G} \varphi\right]=\frac{\alpha_{2}}{\Delta t} \mathbf{D} \cdot \widetilde{\mathbf{u}} \\
\mathbf{u}^{n+1}=\widetilde{\mathbf{u}}-\frac{\Delta t}{\alpha_{2}} \frac{\mathbf{G} \varphi}{\rho^{n}} \\
\mathbf{D} \cdot \mathbf{u}^{n+1}=0 \\
p^{n+1}=p^{n}+\varphi \\
\frac{\alpha_{2} \mathbf{X}^{n+1}+\alpha_{1} \mathbf{X}^{n}+\alpha_{0} \mathbf{X}^{n-1}}{\Delta t}=\beta_{1} \mathbf{U}^{n}+\beta_{0} \mathbf{U}^{n-1} \\
\phi^{n+1} \doteq \phi\left(\mathbf{X}^{n+1}\right)
\end{gathered}
$$

onde os parâmetros $\alpha_{i}, i=0,1,2$ e $\beta_{j}, j=0,1$, são definidos pelas expressões (3.16)-(3.18). Os termos $\mathbf{g}$ e f em (3.72) são dados em (3.24) e (3.25). A velocidade modificada da interface $\mathbf{U}^{k}$, $k=n-1, n, \mathrm{e}$

$$
\mathbf{U}^{k}=\left(\mathcal{S}_{k}^{*} \mathbf{u}\right)(\alpha)+u_{a}^{k}\left(\mathbf{X}^{k}(\alpha)\right) \mathbf{t}
$$

\subsubsection{Força interfacial}

A densidade de força $\mathbf{f}^{n+1}$ para tensão superficial $\sigma$ constante, pode ser obtida ou pela formulação clássica (2.14) ou pela híbrida (2.16), como segue, respectivamente,

$$
\begin{gathered}
\mathbf{f}^{n+1}=\mathbf{f}_{s}^{n+1}=\left(\mathcal{S}_{n+1} \mathbf{F}\right)(\mathbf{x}), \\
\mathbf{f}^{n+1}=\mathbf{f}_{h}^{n+1}=\sigma \kappa_{L} \nabla H_{\epsilon}\left(\phi^{n+1}\right) .
\end{gathered}
$$

Aqui a discretização para $\mathbf{F}$ é dada pelas expressões (3.45)-(3.48). Detalhes da discretização de $\mathbf{f}_{h}$ estão em [92]. A atualização para a função indicadora de fluido $\phi^{n+1}$, baseado na geometria no momento $t=t^{n+1}$, é obtido pela aplicação do algoritmo CPT [31].

\subsubsection{Tensor viscoelástico}

As propriedades materiais atualizadas e o tensor viscoelástico são dados por

$$
\begin{aligned}
& \rho^{n+1}=\rho^{-}+\left(\rho^{+}-\rho^{-}\right) H_{\epsilon}\left(\phi^{n+1}\right), \\
& \mu^{n+1}=\mu^{-}+\left(\mu^{+}-\mu^{-}\right) H_{\epsilon}\left(\phi^{n+1}\right), \\
& \mathbf{S}^{n+1}=I+\left(\mathbf{S}_{p}^{n+1}-I\right) H_{\epsilon}\left(\phi^{n+1}\right),
\end{aligned}
$$

onde $H_{\epsilon}$ é dado por (3.12) e o tensor $\mathbf{S}_{p}^{n+1}$ pode ser determinado pela discretização do Modelo Oldroyd-B ou do FENE-P, feita nas seções (3.4.2) e (3.4.3), respectivamente. 
- Para o Modelo Oldroyd-B:

$$
\mathbf{S}_{p}^{n+1}=\frac{\mathbf{S}_{p}^{n}+\hat{\mathbf{S}}_{p}^{n+2}}{2}
$$

onde, para $k=0,1$,

$$
\begin{aligned}
\hat{\mathbf{S}}_{p}^{n+k+1} & =\hat{\mathbf{S}}_{p}^{n+k}+\Delta \operatorname{tg}\left(\hat{\mathbf{S}}_{p}^{n+k}, \mathbf{u}^{n+1}\right), \\
\mathbf{g}\left(\hat{\mathbf{S}}_{p}^{n+k}, \mathbf{u}^{n+1}\right) & =-\mathbf{u}^{n+1} \cdot \nabla \hat{\mathbf{S}}_{p}^{n+k}+ \\
& +\left(\mathbf{G} \mathbf{u}^{n+1} \cdot \hat{\mathbf{S}}_{p}^{n+k}+\hat{\mathbf{S}}_{p}^{n+k} \cdot\left(\mathbf{G u}^{n+1}\right)^{T}\right)+ \\
& -W_{i}^{-1}\left(\mathbf{S}_{p}^{n+k}-I\right) .
\end{aligned}
$$

- Para o Modelo FENE-P:

$$
\begin{aligned}
\mathbf{C}^{n+1} & =\frac{\mathbf{C}^{n}+\hat{\mathbf{C}}^{n+2}}{2}, \\
\mathbf{S}^{n+1} & =\frac{\mathbf{C}^{n+1}}{1-\left(\operatorname{tr}\left(\mathbf{C}^{n+1}\right) / l^{2}\right)},
\end{aligned}
$$

onde, para $k=0,1$,

$$
\begin{aligned}
& \hat{\mathbf{C}}^{n+k+1}=\hat{\mathbf{C}}^{n+k}+\Delta t \mathbf{h}\left(\hat{\mathbf{C}}^{n+k}, \hat{\mathbf{S}}_{p}^{n+k}, \mathbf{u}^{n+1}\right) \\
& \mathbf{h}\left(\hat{\mathbf{C}}^{n+k}, \hat{\mathbf{S}}_{p}^{n+k}, \mathbf{u}^{n+1}\right)=-\mathbf{u}^{n+1} \cdot \nabla \hat{\mathbf{C}}^{n+k}+ \\
&+\left(\mathbf{G} \mathbf{u}^{n+1} \cdot \hat{\mathbf{C}}^{n+k}+\hat{\mathbf{C}}^{n+k} \cdot\left(\mathbf{G} \mathbf{u}^{n+1}\right)^{T}\right)+ \\
&-W_{i}^{-1}\left(\hat{\mathbf{S}}_{p}^{n+k}-I\right) \\
& \hat{\mathbf{S}}^{n+1}=\frac{\hat{\mathbf{C}}^{n+1}}{1-\left(\operatorname{tr}\left(\hat{\mathbf{C}}^{n+1}\right) / l^{2}\right)}
\end{aligned}
$$

A discretização do termo de advecção em (3.87) e em (3.91), é feita usando ENO3.

\subsubsection{Escoamentos incompressíveis estacionários de fluidos bifásicos}

$$
\begin{gathered}
-\mathbf{G} p^{n+1}+\mathbf{L} \mathbf{u}^{n+1}=\mathbf{f}, \\
\mathbf{D} \cdot \mathbf{L}^{-1} \mathbf{G} p^{n+1}=\mathbf{D} \cdot \mathbf{L}^{-1} \mathbf{f}, \\
\mathbf{u}^{n+1}=\mathbf{L}^{-1} \mathbf{f}+\mathbf{L}^{-1} \mathbf{G} p^{n+1}, \\
\mathbf{D} \cdot u^{n+1}=0, \\
\mathbf{f}_{s}^{n+1}=\left(\mathcal{S}_{n+1} \mathbf{F}\right)(\mathbf{x}), \\
\frac{\alpha_{2} \mathbf{X}^{n+1}+\alpha_{1} \mathbf{X}^{n}+\alpha_{0} \mathbf{X}^{n-1}}{\Delta t}=\beta_{1} \mathbf{U}^{n}+\beta_{0} \mathbf{U}^{n-1}, \\
\phi^{n+1} \doteq \phi\left(\mathbf{X}^{n+1}\right), \\
\mathbf{S}^{n+1}=I+\left(\mathbf{S}_{p}^{n+1}-I\right) H_{\epsilon}\left(\phi^{n+1}\right),
\end{gathered}
$$


onde $\mathbf{f}=-\alpha_{p} \mathbf{D} \cdot \mathbf{S}^{n}-\mathbf{f}_{s}^{n}$.

A solução dos sistemas (3.94)-(3.96) é feita usando o BiCG-Stab. Por outro lado, a solução dos sistemas que envolvem $\mathbf{L}^{-1} \mathbf{Z}$ sendo $\mathbf{Z}$ uma variável vetorial, é realizada com o uso de um esquema matricial e o PETSc. 


\section{Capítulo 4}

\section{Metodologia numérica: AMR e representação matricial das equações discretizadas}

No capítulo anterior, apresentamos a discretização espacial das equações que modelam o escoamento para o caso de malhas eulerianas uniformes. No presente capítulo, estendemos tais discretizações para o caso de malhas compostas com refinamentos adaptativos e, a seguir, estabelecemos a abordagem matricial na qual, agora, a matriz que reflete as discretizações em malhas compostas. A definição da matriz envolve elementos que identificam as incógnitas que precisam ser calculadas e as enumera para assim conseguir o estêncil de interpolações para valores fantasmas e da discretização em cada ponto (centro de célula e meio de aresta). A matriz que representa as equações discretizadas é armazenada em uma estrutura de dados baseada em listas ligadas e emprega o padrão Compressed Sparse Rows (CSR [88]) para armazenar as matrizes esparsas. Definimos a malha composta, a interpolação de valores em células fantasmas e a discretização de operadores diferencias na Seção 4.1. Em seguida, introduzimos na Seção 4.2 a estratégia desenvolvida no presente trabalho para criar a matriz de discretização. Ali, definimos duas estruturas matriciais: uma para armazenar o estêncil da interpolação de valores em células fantasmas e outra para armazenar a representação matricial dos operadores de diferenças finitas empregados. Uma primeira maneira de enumerarmos as células computacionais é proposta e empregada na criação das matrizes. Além disso, propomos a criação de um mapa auxiliar, o qual determina se em uma dada célula computacional há ou não uma equação a ser resolvida (isto é, se a célula computacional é visivel ou invisível). Definimos operações específicas sobre nossas listas ligadas que "traduzem de maneira natural" o estêncil que define a discretização dada nas células computacionais. Para finalizar, na Seção 4.3 mostramos o algoritmo resultante da aplicação do Método de Uzawa às equações de Stokes estacionárias e o Método BiCG-Stab para a solução do sistema discretizado resultante. A implementação retalho-a-retalho do Método BiCG-Stab juntamente à criação das matrizes que representam as equações discretizadas em malhas compostas são um avanço importante obtidos nesta tese. 


\subsection{Domínio euleriano: malha composta}

No presente trabalho, o domínio computacional é dado por um retângulo $\Omega=\left[A_{1}, B_{1}\right] \times\left[A_{2}, B_{2}\right]$ cuja discretização é dada por malhas bloco-estruturadas formadas por blocos retangulares de refinamentos que se adaptam e recobrem regiões específicas do domínio (SAMR). As malhas empregadas em nosso trabalho, denominadas "malhas compostas", são aquelas introduzidas por Berger e Oliger [16, 17, 18]. Mostramos um exemplo na Figura 4.1.
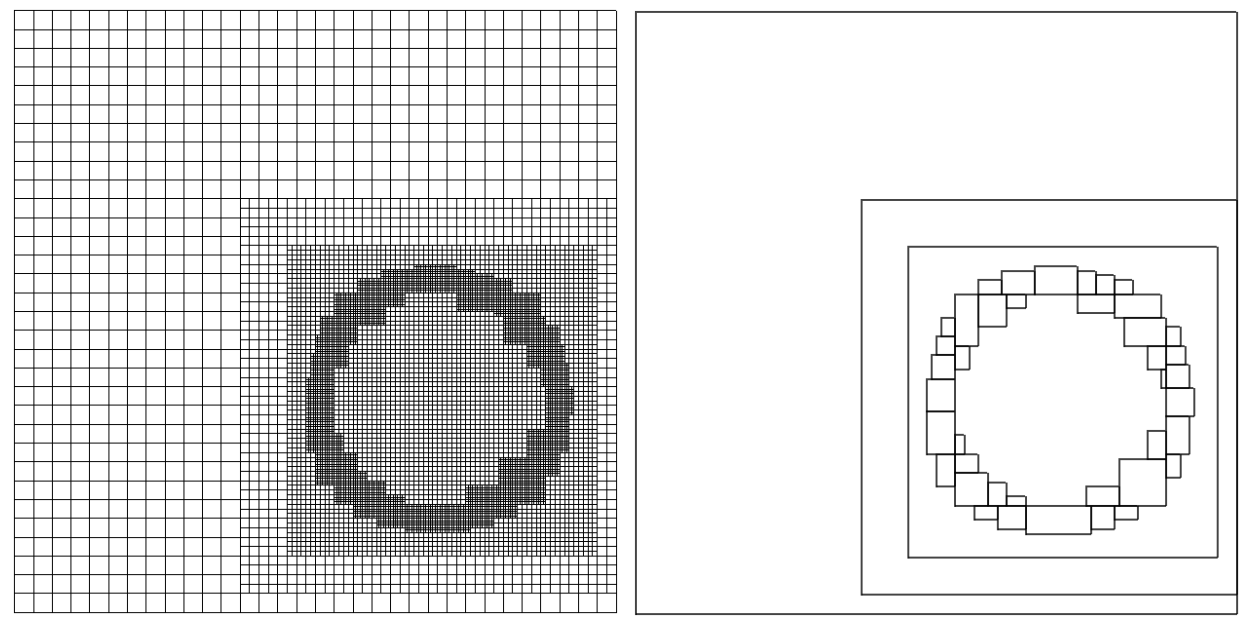

Figura 4.1: Malha composta com 4 niveis de refinamento e sua representação como união de retalhos.

Nessa técnica, refinamentos são obtidos pela união de "retalhos" (patches), cada qual dado por uma malha retangular cartesiana orientada na direção dos eixos coordenados. Uma grande vantagem dessa abordagem é que uma discretização originalmente definida em uma malha cartesiana uniforme pode ser facilmente reutilizada. Refinamentos adaptativos dados por malhas bloco-estruturadas mostraram sua eficácia na simulação da dinâmica de escoamentos incompressíveis $[9,15,16,25$, 29, 32, 78, 79]. No contexto do Método da Fronteira Imersa, o uso destas malhas foi introduzido por Roma, Peskin e Berger [86] no contexto de interfaces imersas e Ceniceros, Roma, Silveira-Neto e Villar no contexto de escoamentos bifásicos [32]. Mais recentemente, empregando a infraestrutura oferecida pela biblioteca SAMRAI ${ }^{1}$ (Structured Adaptive Mesh Refinement Infrastructure), Griffith simulou a dinâmica do escoamento sanguíneo e sua interação com válvulas e musculatura cardíaca [48] e, posteriormente, introduziu uma nova formulação adaptativa para o Método da Fronteira Imersa [47]. A biblioteca SAMRAI é desenvolvida e mantida pelo Lawrence Livermore National Laboratory que realiza algoritmos e software para viabilizar a aplicação dessa técnica de refinamento adaptativo a problemas de larga escala envolvendo física relevantes aos programas financiados pelo Departamento de Energia dos Estados Unidos. Comentaremos em mais detalhes na sequência.

Malhas compostas, além de possuírem implementação computacional relativamente simples quando comparadas a outras técnicas de refinamento adaptativo, oferecem como grande vantagem o fato de possuírem relações de vizinhança de fácil acesso (cada retalho tem uma estrutura natural de indexação). A seleção das regiões onde é necessária uma maior precisão pode ser feita, por exemplo, por intermédio de critérios baseados em propriedades físicas tais como altos gradientes, alta vorticidade e propriedades específicas do fluido. Em uma outra abordagem, Berger e Oliger [17]

\footnotetext{
${ }^{1}$ https://computation.llnl.gov/casc/SAMRAI/
} 
selecionam os pontos que necessitam refinamento com base em uma estimativa do erro de discretização local. Para isto, eles empregam extrapolações de Richardson. Para simulações computacionais de escoamentos multifásicos, uma região natural de grande interesse é a vizinhança da interface de separação entre as fases [111].

Existem pelo menos duas bibliotecas livres que se destacam no uso da técnica SAMR: a SAMRAI (Structured Adaptive Mesh Refinement Application Infrastructure) e a Chombo [3, 33, 79, 80, 109] ("Chombo" significa ferramenta ou container no idioma Swahili, falado em alguns países africanos, e.g. Kenya, Tanzania, Zanzibar, Uganda, República Democrática do Congo e Burundi, entre outros). Essas bibliotecas dão suporte ao paralelismo e permitem o uso de bibliotecas de Álgebra Linear Numérica para a resolução de sistemas associados às discretizações [3, 11, 33, 79].

Aqui, o momento se faz oportuno para alguns comentários sobre nossa experiência com a biblioteca SAMRAI. Inicialmente, tentamos empregá-la para os desenvolvimentos de nosso interesse e que envolviam a técnica SAMR. Para entender a estrutura do SAMRAI, definimos variáveis com funções exatas e tentamos refinar certas regões baseadas em propriedades específicas que definíamos. O que parecia ser simples revelou-se bastante complexo por não termos contato direto com os desenvolvedores da ferramenta. Outra dificuldade surgiu quando tentamos resolver um problema-modelo simples dado por uma equação hiperbólica em uma malha composta com refinamento também no tempo, ou seja, com sincronização de informações dos níveis em diferentes instantes de tempo. O tempo investido na realização destas tarefas, somado à dificuldade do uso da ferramenta e à ausência de um fórum de discussão realmente útil e ativo, terminaram por nos fazer desistir de seu uso. A má experiência com SAMRAI certamente não estimulou tentativas na direção da biblioteca Chombo que, na época, ainda encontrava-se em um estágio de desenvolvimento menos evoluído comparativamente (em 2009).

A partir daí, decidimos utilizar as implementações desenvolvidas por Villar [32, 111] (o "AMR2D") e ideias de Nós [71] (o "AMR3D"). Ambas possuem suporte para o SAMR e métodos multinívelmultigrid implementados para resolver os sistemas lineares associados às discretizações. No presente trabalho, utilizamos a implementação bidimensional tomando o devido cuidado para que a extensão para a versão tridimensional venha a ocorrer de forma natural (de fato, a abordagem matricial para variáveis em centro de células, a saber a correção de pressão, já está sendo transportada para o AMR3D).

A opção pela versão bidimensional foi feita com o intuito de adquirir experiência com a solução de escoamentos viscoelásticos em malhas compostas com estruturas e interfaces de separação imersas. O estudo da solução da equação estacionária de Stokes, com a qual podem ser modelados muitos dos escoamentos viscoelásticos de interesse, levou a optar pela criação de uma matriz para representar as discretizações em malha composta.

Antes de começarmos com a descrição das formas matriciais das equações discretizadas, vamos recordar brevemente como são definidas as malhas compostas bloco-estruturadas e as restrições que devem satisfazer os retalhos retangulares que as constituem, distribuídos dentre os diversos níveis de refinamento. Tais restrições não só permitem que a discretização do problema seja possível como também seja a mais simples possivel. Mais detalhes são encontrados em [16]. 


\subsubsection{Definição da malha composta}

Malhas compostas são definidas por uma sequência hierárquica de níveis de refinamento aninhados e progressivamente mais finos, $l=1,2,3, \ldots, l_{t o p}$. Se $G_{l, k}, k=1,2,3, \ldots, n_{l}$, é um conjunto de retalhos retangulares (patches), todos com os mesmos espaçamentos em cada uma das direções, então o l-ésimo nível de refinamento está dado por

$$
\text { nível } l=\bigcup_{k=1}^{n_{l}} G_{l, k} \text {. }
$$

É importante ressaltarmos que malhas diferentes de um mesmo nível não se sobrepõem, isto é, $G_{l, j} \cap G_{l, k}=\emptyset$, para todo par $1 \leq j, k \leq n_{l} \operatorname{com} j \neq k$.

Retalhos retangulares em diferentes níveis de refinamento devem estar "propriamente aninhados", isto é, eles devem satisfazer a seguintes propriedades [16, 17, 18]:

1. um retalho retangular em um nível fino deve começar e terminar no canto de uma célula do nível imediatamente mais grosso, e

2. as arestas de qualquer retalho em um nível podem apenas tocar células do mesmo nível, do nível imediatamente mais grosso ou do contorno do domínio computacional.

Na Figura 4.2(a), temos uma malha que não se encontra propriamente aninhada; ela não satisfaz a Propriedade 1, enunciada acima, no retalho $G_{2,1}$. Além disso, o retalho $G_{3,1}$ não satisfaz a Propriedade 2. A Figura 4.2(b) mostra uma malha propriamente aninhada.

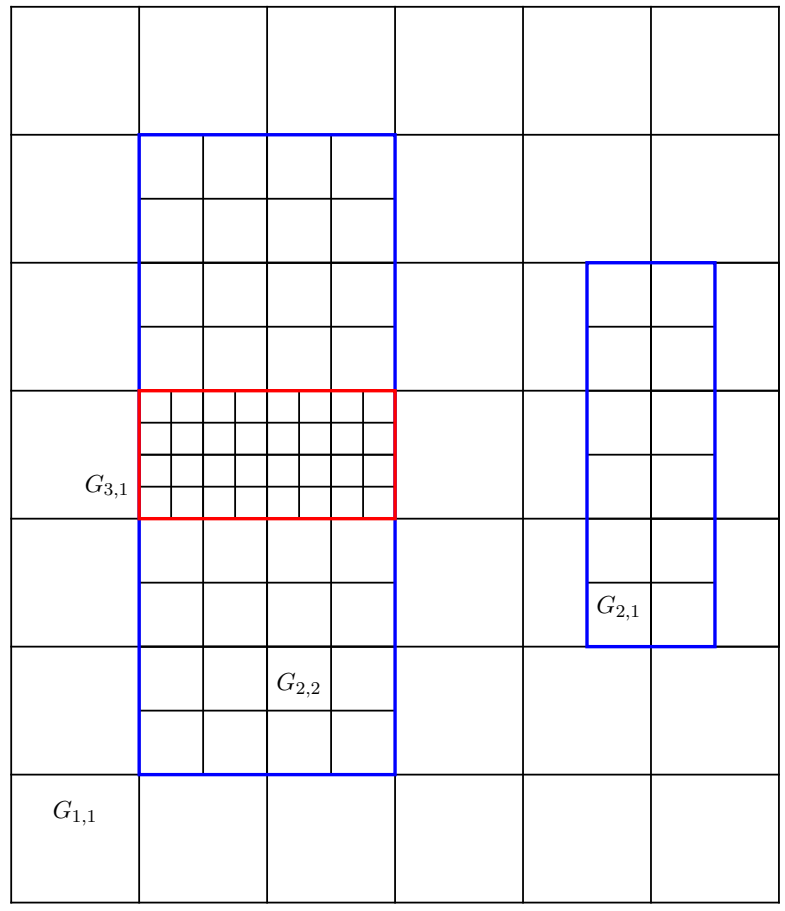

(a). Malha não propriamente aninhada

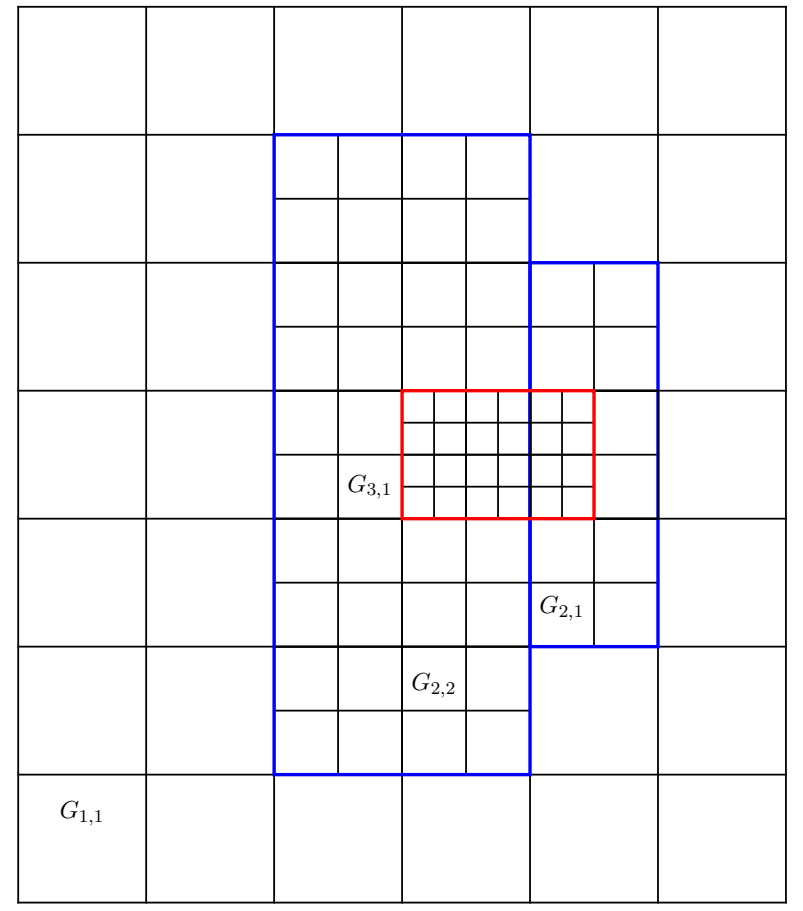

(b). Malha propriamente aninhada

Figura 4.2: Exemplos de malhas propriamente e não propriamente aninhadas. 


\subsubsection{Células fantasmas e seus valores}

Para permitir a comunicação de informação entre os diferentes retalhos da malha (e entre seus diferentes níveis de refinamento) e evitar que o estêncil da discretização de diferenças finitas próximo às interfaces de nível seja "formalmente diferente", definimos ao redor de cada um dos retalhos de malha uma região de células fantasmas. Os valores das variáveis nas células fantasmas são calculados por meio de interpolações polinomiais e fornecem uma aproximação do valor das variáveis naquela posição, definindo assim condiçôes de contorno em cada retalho. A Figura 4.3 mostra uma malha com dois níveis: o nível de base e mais um nível de refinamento. O segundo nível é composto por três retalhos de malha, $G_{2,1}, G_{2,2}$ e $G_{2,3}$. A Figura 4.3 mostra os tipos de aproximações que podem acontecer para se obter os valores armazenados nas células fantasmas, ilustradas no bloco $G_{2,2}$.

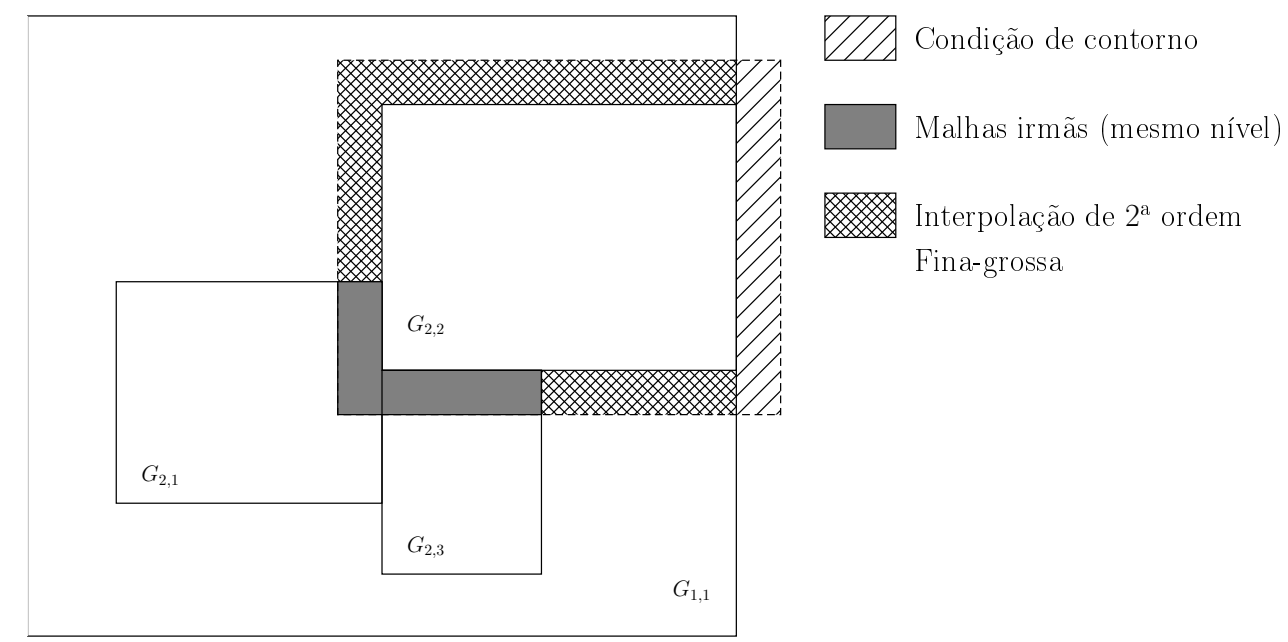

Figura 4.3: Aproximação para células fantasmas do retalho $G_{2,2}$.

A obtenção de valores nas células fantasmas definidas ao redor de cada retalho de cada nível de refinamento depende de interpolações que envolvem valores em células computacionais pertencentes a níveis de refinamento diferentes. Os pesos destas interpolações dependem da localização das variáveis nas células computacionais (centro de célula ou meio de aresta) e da ordem que se deseja para a aproximação. Por sua vez, tal ordem de aproximação está ligada à discretização dos operadores diferenciais e de como aproximamos as variáveis em células recobertas. Para aproximar os valores de células fantasmas em regiões com interface fina-grossa, usamos tipicamente interpolação com polinômios quadráticos [85], como discutiremos em detalhes mais adiante. Na Figura 4.6, mostramos um exemplo para obter tal interpolação.

Em uma malha composta, as variáveis que são obtidas por meio da resolução de uma equação diferencial estão definidas nas células de um retalho que não estão recobertas por outros retalhos de outros níveis de refinamento. Chamamos as células que contêm variáveis que provém da resolução de equações discretizadas de células visiveis pois são, de fato, literalmente aquelas que se vê quando se traça a figura da malha composta empregada. Todas as aproximações obtidas para variáveis em células visíveis dependem da discretização das equações usadas e do valor de variáveis pertencentes também a células visíveis.

A aproximação das variáveis definidas em células recobertas por uma malha do nível imediatamente mais fino, acima, pode ser definida usando uma interpolação de variáveis no nível mais fino. 
As interpolações que empregamos nesse caso, tipicamente são de dois tipos: ou a média dos valores das células do nível acima ou uma interpolação polinomial cúbica. Chamamos de células invisiveis às células recobertas e as células fantasmas de todos os retalhos de todos níveis de refinamento. $\mathrm{Na}$ Figura 4.4, mostramos para uma malha composta a região que corresponde a células visíveis, na cor cinza, e a região que corresponde a células recobertas para esta malha na cor branca.

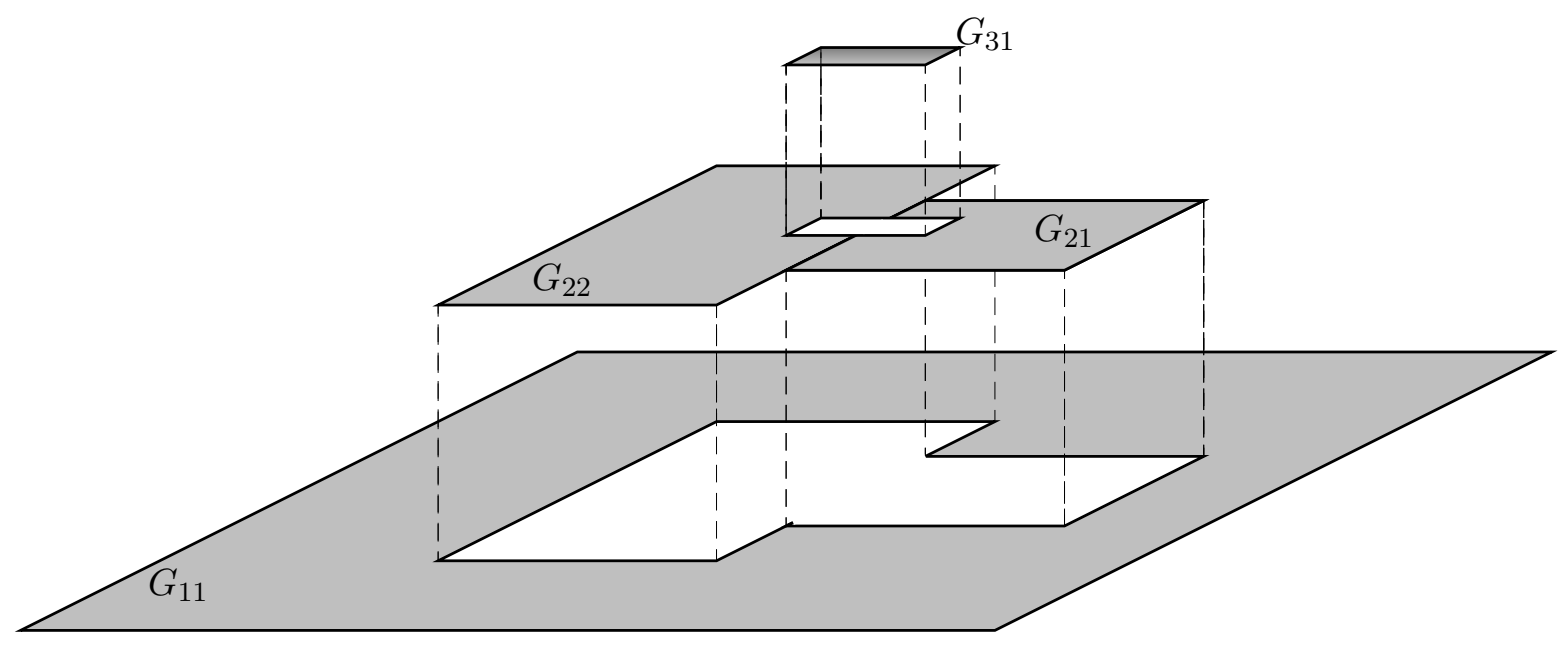

Figura 4.4: Células visiveis e recobertas na malha composta.

A relação entre células visíveis e as variáveis está dada em que as variáveis definidas em células visíveis são as variáveis que desejamos aproximar e para as quais os sistemas lineares determinam uma solução.

Cada um dos retalhos que forma a malha composta pode ser tratado como uma malha cartesiana uniforme. Em cada um deles, é possível fazer uma aproximação por diferenças finitas. Para obtermos aproximações corretas ao usarmos operações retalho-a-retalho e nível-a-nível, os valores em todas as células visíveis e invisíveis devem estar apropriadamente definidos.

Os passos para definir corretamente uma variável em uma malha composta, isto é, definir seus valores em células fantasmas e em células recobertas, são os seguintes:

Passo 1. Em células recobertas, os valores são definidos como a média aritmética dos valores finos, no nível de refinamento acima, que a recobrem;

Passo 2. Extrapolação-interpolação cúbica de valores no contorno de cada retalho de um dado nível para as células recobertas do nível mais grosso abaixo;

Passo 3. Interpolação fina-grossa;

Passo 4. Preenchimento de valores em células fantasmas sobrepostas por "malhas irmãs", isto é, injeção de valores diretamente de retalhos de mesmo nível, vizinhos;

Passo 5. Condição de contorno no bordo do domínio computacional.

Os passos 1 e 2 definem de maneira apropriada os valores nas células recobertas. Do passo 3 até o passo 5, definimos os valores de todas as células fantasmas. Estes passos são os que foram mostrados na Figura 4.3. 
Destacamos que a solução de sistemas lineares com o método multinível-multigrid [3, 32, 33, 71] está relacionada à ideia de uma matriz local para cada retalho. A solução final desejada é encontrada nas células visíveis. Entretanto, ao fazer a solução do sistema usando uma estragégia de varreduras retalho-a-retalho estamos aproximando também a solução nas células recobertas. Isto não é ineficiente, se comparado com a quantidade de condicionais que precisaríamos empregar para identificar os tipos de células, garantir suas condições de contorno e decidir calcular ou não uma incógnita. Estas dificuldades não existiriam se tivéssemos uma matriz global para todas as células visíveis de todos níveis. Nesse caso, a grande dificuldade está em relacionar o estêncil da discretização com todas as interpolações necessárias para obter os valores em cada célula fantasma, dadas do passo 1 até o passo 5 .

Para realizar a extrapolação-interpolação cúbica, por exemplo, empregamos 16 células vizinhas da célula recoberta que desejamos interpolar. Essa interpolação para células recobertas, definidas no contorno dos retalhos de malha, não é comum de ser encontrada na literatura relacionada à técnica SAMR [3, 33, 79, 109].

Para obtermos esta aproximação de quarta ordem, primeiro fazemos uma extrapolação cúbica para as células fantasmas do nível fino como na Figura 4.5(a), depois interpolamos até a aresta relacionada diretamente com a posição a ser aproximada como na Figura 4.5(b) e, finalmente, uma última interpolação linear define o valor da variável coberta.

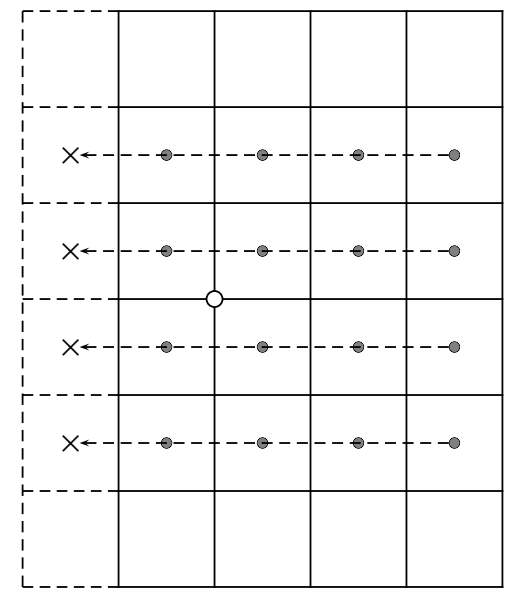

(a)

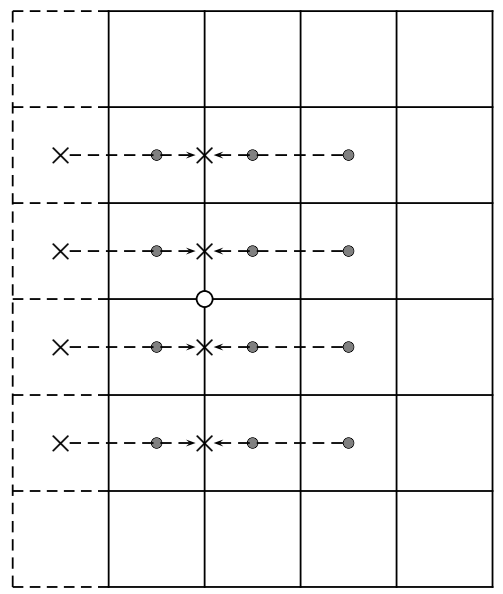

(b)

Figura 4.5: Interpolação cúbica para célula grossa: (a) Extrapolação cúbica, (b) Interpolação cúbica.

A extrapolação-interpolação cúbica para as células recobertas ao redor de cada retalho do nível mais fino é necessária pela experiência adquirida e acumulada ao longo do tempo (e.g. [71, 111]). Nas regiões denominadas "T-junctions", isto é os cantos formados pelos retalhos de diferentes tamanhos que compartilham arestas, a ordem da aproximação degenerava. Isto implicou no aumento da precisão das células recobertas por estas regiões, incluindo informações de ambos os retalhos. Identificar a localização de T-junctions pode ser mais caro do que simplesmente interpolar o contorno de células recobertas em todo os retalhos de cada nível de refinamento.

A interpolação fina-grossa que empregamos é dada por duas aproximações quadráticas: uma que relaciona o nível grosso e outra que relaciona a interpolação do nível grosso com as células no nível fino. A Figura 4.6 mostra um exemplo de interpolação quadrática na interface fina-grossa. A interpolação fina-grossa é uma operação que define valores em célula fantasmas com terceira ordem 
de precisão.

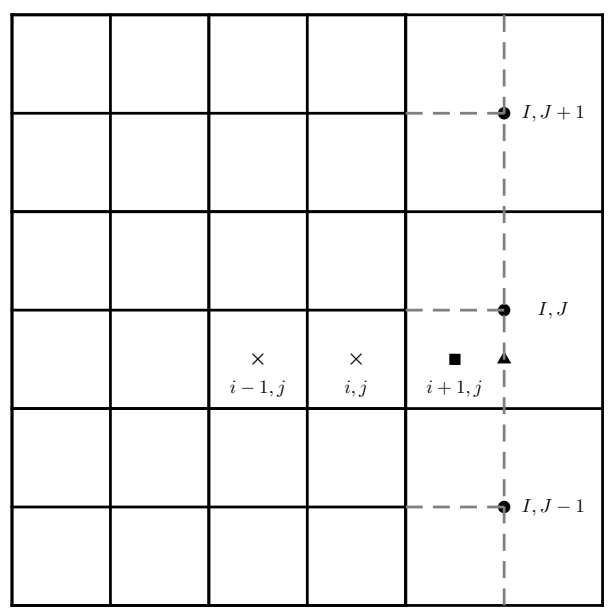

Interpolação quadrática

$$
\varphi_{\mathbf{\Lambda}}=\frac{5}{32} \varphi_{I J-1}+\frac{15}{16} \varphi_{I J}-\frac{3}{32} \varphi_{I J+1}
$$$$
\varphi_{i+1 j}=\frac{-1}{5} \varphi_{i-1 j}+\frac{2}{3} \varphi_{i j}+\frac{8}{15} \varphi_{\boldsymbol{\Lambda}}
$$

$$
\begin{aligned}
\varphi_{i+1 j} & =\frac{-1}{5} \varphi_{i-1 j}+\frac{2}{3} \varphi_{i j}+\frac{8}{15}\left(\frac{5}{32} \varphi_{I J-1}+\frac{15}{16} \varphi_{I J}-\frac{3}{32} \varphi_{I J+1}\right) \\
\varphi_{i+1 j} & =\frac{-1}{5} \varphi_{i-1 j}+\frac{2}{3} \varphi_{i j}+\frac{1}{12} \varphi_{I J-1}+\frac{1}{2} \varphi_{I J}-\frac{1}{20} \varphi_{I J+1}
\end{aligned}
$$

Figura 4.6: Interpolação fina-grossa na célula $(i+1, j)$.

Outros autores realizam esta interpolação de maneira linear e não quadrática e nas regiões com T-junctions fornecem uma interpolação particular para não incluir células recobertas [42, 64, 83]. Aqui, para incrementar a ordem das discretizações localmente, mantemos a interpolação fina-grossa de maneira quadrática.

A implementação AMR2D tem suporte para definir os valores em todas as células fantasmas da malha. Entretanto, foram necessárias algumas alterações para estender o uso das rotinas de cálculo existentes para outras situações. Em particular, podemos citar:

- a interpolação fina-grossa: os valores fantasmas oriundos da condição de contorno e de células irmãs precisaram ser redefinidos antes da execução dessa interpolação. Nas rotinas originais, havia um problema no cálculo das condições de contorno feitos antes de realizarmos a interpolação fina-grossa. Havia também um outro problema relacionado ao recobrimento por valores oriundos de células irmãs, os quais eram alterados depois das extrapolações cúbicas;

- a escolha de células que tocavam a fronteira do domínio físico destinadas a serem preenchidas com a condição de contorno. Este era um problema que só afetava malhas com mais de uma camada de células fantasmas, próximas à fronteira do domínio.

Antes de realizarmos tais alterações no cálculo de valores em células invisíveis, não obtínhamos convergência ao tentarmos resolver escoamentos estacionários via Método de Uzawa em uma implementação preliminar baseada na estratégia de varredura retalho-a-retalho, sem o uso de qualquer representação matricial das equações discretizadas. No caso em que são necessárias mais do que uma camada de células fantasmas (e.g. na implementação do esquema ENO de terceira ordem para o qual são necessárias três camadas), a necessidade dessas alterações tornava-se ainda mais evidente, produzindo a implementação original efeitos ainda mais salientes e dramáticos no que diz respeito a comportamentos obscuros de ordem e de convergência numérica. 
Um entendimento mais aprofundado, adquirido ao longo do tempo e com o desenvolvimento do corrente trabalho, possibilitou a elaboração de tais alterações na implementação original das rotinas de cálculo de valores fantasmas. Foi essencial o uso de formas matriciais para a obtenção dos valores fantasmas para percebermos o que deveria ser feito na maneira convencional como eles vinham sendo obtidos, empregando a estratégia baseada em varreduras retalho-a-retalho, nível-a-nível. É possível que estes detalhes de implementação tenham passado desapercebidos em processos de depuração anteriores. No presente trabalho, introduzimos testes de convergência e de investigação de ordem bem mais restritivos, empregando malhas compostas estáticas as quais possuem um número grande de retalhos em posições variadas e arbitrárias, espalhados em vários níveis de refinamento. No passado, tipicamente, usávamos apenas dois níveis de refinamento dados por um total de, quando muito, meia dúzia de retalhos de malha.

\subsubsection{Discretização dos operadores diferenciais na malha composta}

Suplementamos aqui a informação sobre a discretização no espaço dos operadores diferenciais, fornecida na Seção 3.1.1 para malhas uniformes. Em malhas compostas tal discretização está dada por:

- Gradiente: após a atualização dos valores fantasmas, aproximamos em cada retalho de cada nível o gradiente com a discretização (3.1);

- Divergente: aproximamos em cada retalho de cada nível o divergente com a discretização (3.4);

- Produto interno:

$$
\left\langle\varphi_{1}, \varphi_{2}\right\rangle=\sum_{i=1}^{n_{x}} \sum_{j=1}^{n_{y}} \varphi_{1, i j} \varphi_{2, i j} w_{i j} \Delta x \Delta y
$$

onde $w_{i j}$ é 0 se a célula $(i, j)$ é invisível e 1 em caso contrário.

Esta é uma operação que passa por todas as células de todos os níveis. Logo, para $\varphi_{1}, \varphi_{2} \in G_{l, k}$ com $l=1, \ldots, l_{\text {top }}$ e $k=1, \ldots, n_{l}$ temos

$$
\left\langle\varphi_{1}, \varphi_{2}\right\rangle_{l}=\sum_{l=1}^{l_{\text {top }}} \sum_{k=1}^{n_{l}}\left\langle\varphi_{1}, \varphi_{2}\right\rangle ;
$$

- Atualização de valores nas arestas compartilhadas de malhas irmãs: acertamos os valores de meio de arestas nas células fantasmas de retalhos que se tocam compartilhando arestas de seus bordos (Fillsister_Face);

- Média de variáveis: para todas as variáveis recobertas, se uma variável $\varphi_{i j}$ está definida em uma célula recoberta por um nível mais fino, então $\varphi_{i j}$ é a média das correspondentes variáveis do nível fino (Average_Var);

- Cálculo de fantasmas: aproxima as células fantasmas para todos os retalhos de cada nível, seguindo os passos (1)-(5) dados na Seção 4.1 (Calc_Ghost); 
- A Definição correta de uma variável localizada em uma aresta consiste de três passos: 1. Fillsister_Face, 2. Average_var e 3.Calc_Ghost (estas operações são dadas por correct_Definevar).

Os sistemas lineares, como por exemplo aqueles resultantes da discretização da equação de Poisson, podem ser solucionados por diferentes métodos, e.g. o Método de Gauss-Seidel e o multinívelmultigrid [29, 32, 85, 102, 104]. No caso de fluidos bifásicos, quando alguma das propriedades materiais do fluido apresenta um "salto acentuado", a resolução da equação para a correção de pressão (3.29) por estes métodos apresenta problemas, convergindo lentamente ou divergindo [111]. Isto vem corroborar a necessidade de estudarmos outras formas para métodos numéricos que possam ser empregados na estrutura dada pelas malhas compostas resolver tais equações.

$\mathrm{Na}$ tentativa de obtermos implementações mais robustas, de obtermos a resolução da equação estacionária de Stokes empregando o Método de Uzawa (baseado no BiCG-Stab), de resolver a dificuldade numérica introduzida quando há saltos elevados nas propriedades materiais (especialmente na massa específica), de entender melhor o comportamento e as características de sistemas lineares oriundos de discretizações em malhas do tipo SAMR (e.g. raio espectral e número de condição, entre outras), de obter acesso a diferentes solvers para sistemas lineares via bibliotecas numéricas gratuitas (e.g. PETSc), a paralelismo e a precondicionamentos do sistema linear, pensamos na criação da representação matricial "explícita" das equações discretizadas em malhas adaptativas bloco-estruturadas.

A obtenção da estrutura matricial que reflete uma discretização por diferenças finitas em uma malha composta está relacionada à localização das incógnitas e à estratégia de enumeração das células visíveis. Ao se empregar tão fortemente valores fantasmas definidos nas células invisíveis na discretização, o estêncil da interpolação é fortemente afetado e isto se reflete na matriz (e no seu processo de construção). Por este motivo, necessitamos de uma estratégia de construção da matriz que permita obter os valores ou os pesos empregados na interpolação dos valores fantasmas definidos nas células invisíveis e as posições de suas correspondentes variáveis.

\subsection{Estrutura matricial}

O uso da técnica SAMR nas discretizações espaciais nos permite concentrar o esforço computacional em regiões do domínio de especial interesse. Em tal técnica, temos acesso rápido à informação de vizinhos - está é uma de suas grandes vantagens (além de serem facilmente implementadas).

No trabalho presente, desenvolvemos uma maneira de obter a representação matricial de discretizações em malhas compostas. A abordagem matricial de problemas definimos em malhas SAMR é incomum, não havendo nenhuma ou pouquíssima documentação de seus aspectos algorítmicos. Inicialmente, Ewing [41, 42] apresentou matrizes em malhas compostas, as quais chamou "Compositegrid-Matrix", para a solução da equação de Poisson, determinando propriedades específicas sobre os valores próprios da matriz, entre outros. Para estas matrizes, Ewing determinou pesos bem específicos quando uma célula computacional é refinada em três novas células. Recentemente, Pletzer [83] propôs uma técnica para obtenção do estêncil na interpolação fina-grossa para uma interpolação linear ou quadrática. Ambos os trabalhos diferem daquele apresentado aqui, por serem específicos para o caso de variáveis no centro de célula, não usarem interpolações cúbicas que ajudam na interpolação fina-grossa continuar de segunda ordem em regiões como as T-junctions. Tanto quanto 
pudemos constatar, investigando não só a literatura como também fóruns de discussão especializados (SAMRAI e Chombo), o trabalho aqui apresentado é completamente original não só com relação aos graus dos interpolantes e formas de obtenção de valores fantasmas, como também no tratamento matricial de variáveis de meio de aresta.

No contexto de malhas compostas, as bibliotecas citadas anteriormente (SAMRAI e Chombo), não usam uma matriz explicitamente obtida, de forma global, para representar os sistemas lineares da malha composta. Por exemplo, Chombo fornece opções com matrizes locais oriundas da discretização em cada retalho [3]. SAMRAI e Chombo empregam na resolução dos sistemas lineares as bibliotecas PETSc e KINSol ${ }^{2}$ (KINSol é uma das bibliotecas que contém o SUNDIALS - SUite of Nonlinear and DIfferential/ALgebraic equation Solvers, desenvolvido pelo Center for Applied Scientific Computing do Lawrence Livermore National Laboratory nos Estados Unidos). As bibliotecas numéricas PETSc e KINSol usam classes de vetóres próprios e realizam o produto matriz-vetor sem utilizar uma forma explícita da matriz [3, 33, 79, 80, 109].

Para conseguir obter a estrutura matricial que representa uma discretização em uma malha SAMR, precisamos armazenar o estêncil de interpolações nas células recobertas e fantasmas. Logo, antes de definir a matriz é necessário termos acesso, para cada célula envolvida em uma discretização, qual é o seu estêncil ou, em uma outra maneira de se colocar, qual é o "caminho de células" na aproximação do valor que ela tem.

Definimos aqui duas estruturas: uma que armazena o estêncil das interpolações de células fantasmas e células recobertas que definem corretamente uma variável e uma outra que armazena o estêncil da discretização respeitando o tipo de célula computacional (se é uma célula definida por condição de contorno, por aproximação cúbica, por média aritmética, por interpolação fina-grossa ou por ser uma célula com o valor de um retalho de uma malha irmã).

A estrutura de dados que empregamos para armazenar a relação de pesos utilizados na interpolação de uma célula fantasma e do estêncil oriundo de uma discretização, ambos com os correspondentes índices das células, segue a ideia do armazenamento de matriz esparsa conhecida como Compressed Sparse Rows, ou CSR [88]. Com este formato de armazenamento, em cada linha da matriz esparsa só são armazenados para cada linha da matriz os elementos diferentes de zero e os índices da coluna correspondente na matriz. O objetivo principal deste tipo de estrutura de dados é diminuir a quantidade de memória usada no armazenamento e garantir operações na ordem da quantidade de elementos diferentes de zero por linha.

Em nosso caso, da mesma forma que no CSR, para acompanhar o estêncil na interpolação de células fantasmas ou de uma discretização do operador diferencial, armazenamos para cada célula os pesos relacionados à interpolação das células fantasmas e da discretização do operador diferencial e os índices segundo a enumeração das células correspondentes. Este é um processo iterativo no qual uma célula pode ter um número de índice associado a ela ou pode ter uma coleção de pesos e índices de uma outra interpolação feita.

Para o armazenamento de cada célula da estrutura que corresponde à sua interpolação, usamos uma lista ligada [89], onde cada nó $k$ da lista contém o valor do peso $w_{k}$, o índice da célula $I d_{k}$ e um ponteiro para o próximo nó da lista, como na Figura 4.7.

A lista ligada que usamos possui cabeça e está ordenada pelos índices $I d$ das células. Na Seção 4.2.2, temos uma representação desta lista. Nas listas ligadas, a busca, a inserção e a re-

\footnotetext{
${ }^{2}$ https://computation.llnl.gov/casc//sundials/main.html
} 


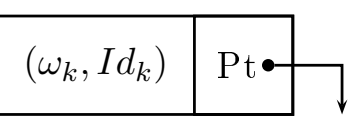

Figura 4.7: Representação de um nó da lista ligada matricial.

moção são na ordem da quantidade de elementos da lista [89]. Usamos essa lista ligada na estrutura matricial para o armazenamento das interpolações de células fantasmas e na estrutura matricial que armazena o estêncil das discretizações. A cabeça desta lista armazena informações específicas da célula com a qual está relacionada a lista, tais como o índice da própria célula, o apontador para o primeiro elemento da lista, a quantidade de elementos da lista (elementos não nulos nnz na linha da matriz que representa a lista) e o valor de condição de contorno denotado por $V a l_{-} B C$.

$\mathrm{O}$ valor $\mathrm{Val} \_\mathrm{BC}$ sempre será zero quando a condição de contorno é periódica ou de Neumann. No caso de condição de Dirichlet, o valor $V a l_{-} B C$ pode ser diferente de zero e está relacionado com o valor exato na fronteira do domínio dependendo da interpolação relacionada a ele. Temos que ter um cuidado especial com esta variável porque sem ela podemos não obter novamente a aproximação desejada em uma interpolação. Por exemplo, a aproximação com condição de contorno de Dirichlet para uma variável $\phi$ definida no centro de uma célula e que está na fronteira esquerda do domínio físico (na posição de célula fantasma) é

$$
\phi(x-\Delta x)=-\phi(x+\Delta x)+2 f(x),
$$

onde, neste caso, $V a l_{-} B C=2 f(x)$.

Esta representação é válida tanto para a estrutura de armazenamento para seguir o caminho de interpolação de células fantasmas, como para o armazenamento do estêncil da discretização.

Pela definição que estamos usando na inserção e remoção de elementos da lista, em que logo depois de cada uma destas operações a lista continua ordenada pela chave $I d$ de cada nó, concluímos que todos os elementos da lista têm o valor $I d$ diferente. Quando inserimos um novo elemento na lista, se o índice dele não se encontra na lista um novo nó sera criado na posição que lhe corresponde, mas se o índice já está na lista então será armazenado um novo peso $w$ com a soma deles e nenhum nó é criado.

No momento, empregamos estruturas de dados baseadas em listas ligadas pois não tínhamos ideia da quantidade de células que podiam estar envolvidas nas interpolações tais como, por exemplo, quantas células de quantos níveis poderiam estar envolvidas na definição de um estêncil correspondente a uma interpolação (para valores em células invisíveis) ou na discretização de um determinado operador diferencial. Com a experiência adquirida, esta estrutura poderá ser mudada para outra baseada no uso de vetores (muito mais adequada do ponto de vista de comunicação em ambientes de memória distribuída em futuras versões paralelas).

Ao longo do desenvolvimento da representação matricial, inicialmente trabalhamos com uma única estrutura que armazenava primeiro o estêncil da discretização do operador diferencial desejado para todas as células visíveis e, na sequência, o estêncil dos valores definidos para a variável considerada nas células invisíveis. Esta estrutura era vetorial e a dimensão dependia da quantidade de células visíveis e de células invisíveis e necessitava de uma relação única para células visíveis e outra para as invisíveis. Para casos particulares, com dois níveis de refinamento e variáveis definidas no centro de células, obtivemos sucesso com esta estratégia. Entretanto, quando voltamos nossa 
atenção a variáveis definidas no meio de arestas, esta estrutura tornou-se complexa e sua implementação inviável. Uma outra desvantagem desta estrutura inicial era que a mesma teria que ser recriada completamente para casos tridimensionais e novos algoritmos teriam que ser concebidos para obtermos a relação entre as células visíveis e invisíveis.

Finalmente, obtivemos sucesso em ambos os casos, para variáveis de centro de célula e de meio de aresta, com a estrutura que usamos atualmente. O armazenamento das listas ligadas em cada célula acompanha a estrutura de dados das variáveis no AMR2D. Então temos uma relação onde o índice da posição de cada variável na malha é o mesmo índice usado para o armazenamento da lista ligada relacionada a essa célula. Esta estrutura teve grandes vantagens, sendo a principal a extensão imediata para o caso tridimensional, dado que as estruturas de dados usadas no AMR2D e no AMR3D são semelhantes [71, 111]. Aqui, como a lista ligada é independente da dimensão do problema, as estruturas de dados que criamos são usadas no caso tridimensional sem ser necessária qualquer mudança significante, dado que esta estrutura acompanha as células da malha.

A base da nossa ideia de armazenamento de listas ligadas é identificar corretamente as células visíveis e invisíveis e criar uma enumeração única para células visíveis. Nós criamos uma enumeração que parece ser "natural", mas a estrutura aceita qualquer estratégia de enumeração, mesmo fornecidas como um "dado de entrada", desde que não se alterem as definições de "visibilidade" e "invisibilidade" das células.

\subsubsection{Mapeamento de células e enumeração}

Para traduzirmos a estrutura hierárquica do SAMR - dada por intermédio de níveis de refinamento e de retalhos de malhas cartesianas - para uma estrutura matricial, necessitamos que ambas reconheçam células visiveis e células invisiveis. Com esta finalidade, construimos um mapa binário relacionado com a posição de cada variável nas células computacionais, o qual denominamos de mapa de visibilidade. O mapa de visibilidade retorna 1 se a célula computacional é visível e 0 caso contrário. Precisamos criar um mapa para cada tipo de variável: um para variáveis localizadas no centro de célula, outro para variáveis de meio de aresta na direção $x$ e, outro ainda, para variáveis em meio de aresta na direção y (na verdade, nesses dois últimos casos, deveríamos dizer que teremos então "mapas de visibilidade de arestas" - entretanto, arriscaremos o abuso de linguagem pois acreditamos que não corremos o risco de provocar confusão).

Quando tratamos do caso em que as variáveis estão definidas no centro de célula, todas as células fantasmas e todas as células recobertas, isto é, todas as células invisíveis, terão valor zero. Para variáveis definidas em meio de aresta, o mapa devolve valor zero para arestas que têm interseção não nula com arestas pertencentes a algum retalho de alguma malha de algum nível mais fino que o recobre. Além disso, quando dois retalhos em um mesmo nível compartilham uma aresta, consideramos apenas uma delas como sendo visível - a mesma aresta, mas contida nesse outro retalho de mesmo nível, recebe o valor zero no mapa de visibilidade (de arestas).

As condições de contorno afetam o mapa de visibilidade, especialmente no caso em que ele relaciona variáveis definidas em meio de aresta com a condição de contorno periódica (veja detalhes no Apêndice B). Quando temos a condição de contorno de Dirichlet e o mapa diz respeito, por exemplo, às variáveis definidas em meio de arestas na direção $x$ (direção $y$ ), o valor das variáveis sobre as arestas a oeste ou sobre as arestas a leste da fronteira do domínio físico (arestas ao sul ou arestas ao norte, respectivamente) é conhecido e, por isso, poderiam ser considerados como 
células invisíveis (pois não há incógnita definida ali e, portanto, não há equação a ser resolvida). Entretanto, não é assim que fazemos aqui. Nossa intenção é a de traduzir o mais fielmente possível o algoritmo de cálculo de valores em células fantasmas implementado no AMR2D [111] para poder identificar eventuais pontos nos quais podemos melhorá-lo e estendê-lo para outras aplicações. Na implementação do AMR2D, os valores nas arestas no caso de condições de contorno de Dirichlet são considerados, primeiro, como "valores fantasmas" a serem determinados e, como tais, são aproximados primeiro por interpolações. Em seguida, eles são corrigidos (sobre-escritos) empregando os valores exatos conhecidos. Na implementação original, esta estratégia foi adotada para se evitar perguntas, o uso de comandos de seleção condicionais, para identificar se uma aresta está ou não no bordo do domínio computacional. Tais perguntas tornariam o programa menos eficiente.

Para ilustrar o mapa de visibilidade, consideremos a malha composta com três níveis de refinamento dada na Figura 4.2(b) e a enumeração em cada nível dos retalhos da malha como na Figura 4.8 .

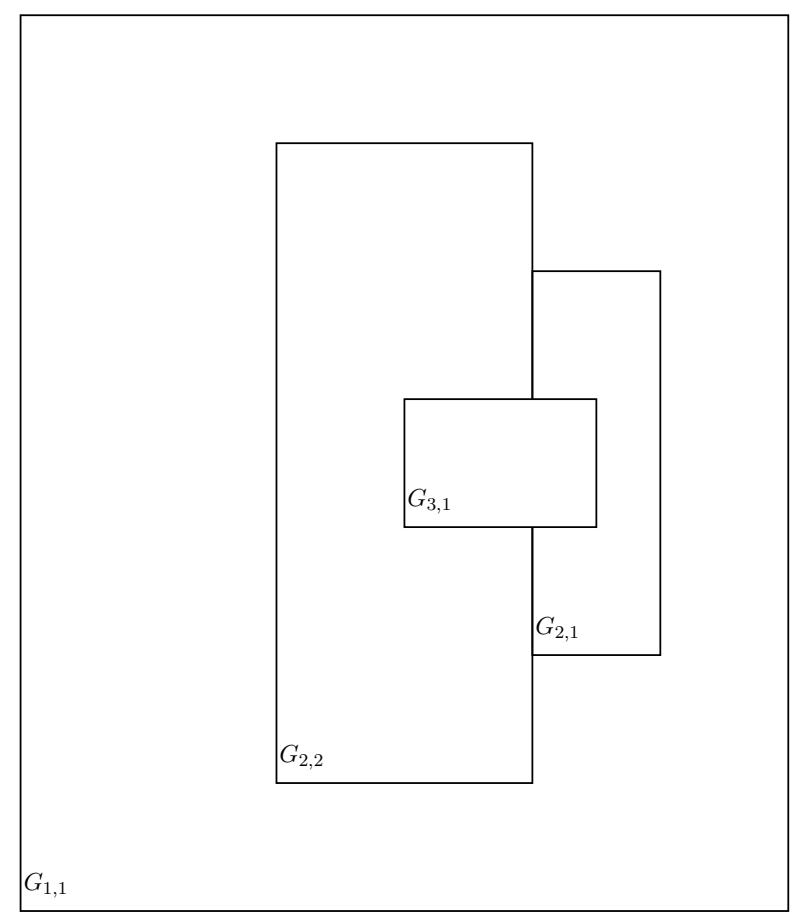

Figura 4.8: Enumeração de retalhos.

Para as variáveis de centro de célula com condição de contorno de Dirichlet (ou de Neumann) da malha dada na Figura 4.2(b), temos o mapa de visibilidade, representado nível a nível, nas figuras 4.9, 4.10 e 4.11.

Para o nível de base, representado na Figura 4.9, vemos que toda a região recoberta pelo nível mais fino acima (região cujo contorno está definido em negrito), tem valor "zero".

O nível de base é cercado por uma "moldura" de células fantasmas (uma moldura de espessura igual a um) cujos valores vêm de condições de contorno. Nosso mapa de visibilidade, nessa moldura, retorna também o valor zero. No segundo nível de refinamento, representado na Figura 4.10, nosso mapa retorna "um" para os centros de célula no interior dos retalhos apenas para as células que não estão recobertas. Centros de célula pertencentes às células fantasmas no contorno de cada retalho, 
onde os valores são determinados por interpolações fina-grossa ou por valores injetados oriundos de células de malhas irmãs, sempre têm valor zero. Por último, mostramos na Figura 4.11 o nível mais fino de refinamento, no qual todos os centros de células são visíveis e, portanto, recebem o valor "um". Como esperado, os centros de célula das células fantasmas retornam o valor "zero".

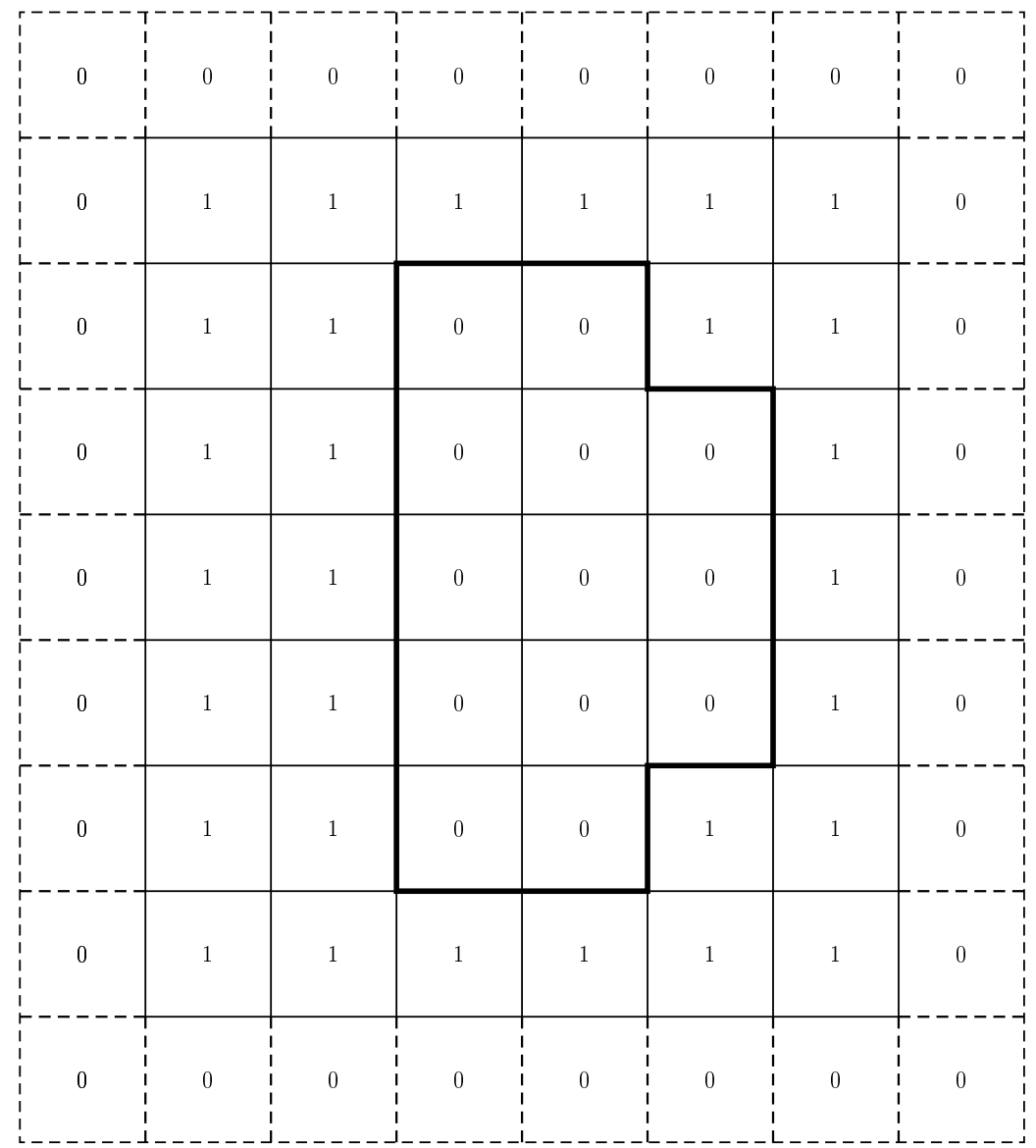

Figura 4.9: Mapa de visibilidade nivel base no centro da célula.

Para decidir se uma aresta é visível ou invisível, temos que ter um cuidado adicional, desnecessário no caso de centros de célula. Retalhos de malhas de um mesmo nível podem compartilhar um conjunto de arestas onde essas malhas, se justapostas, se tocam. Mesmo se essas arestas não estiverem recobertas por nenhuma malha de um nível mais fino, as arestas que forem compartilhadas por mais de um retalho de malha devem receber o valor "zero" para uma das malhas que as contêm. Para a outra malha, a aresta compartilhada receberá o valor "um". Em outras palavras, uma aresta compartilhada por dois retalhos diferentes pertencentes ao mesmo nível de refinamento, só pode estar representada uma única vez na matriz. Por isto, a convenção que assumimos no caso de arestas compartilhadas é que sempre a aresta que pertence ao retalho a leste (correspondentemente, ao retalho ao norte) será a visível. 

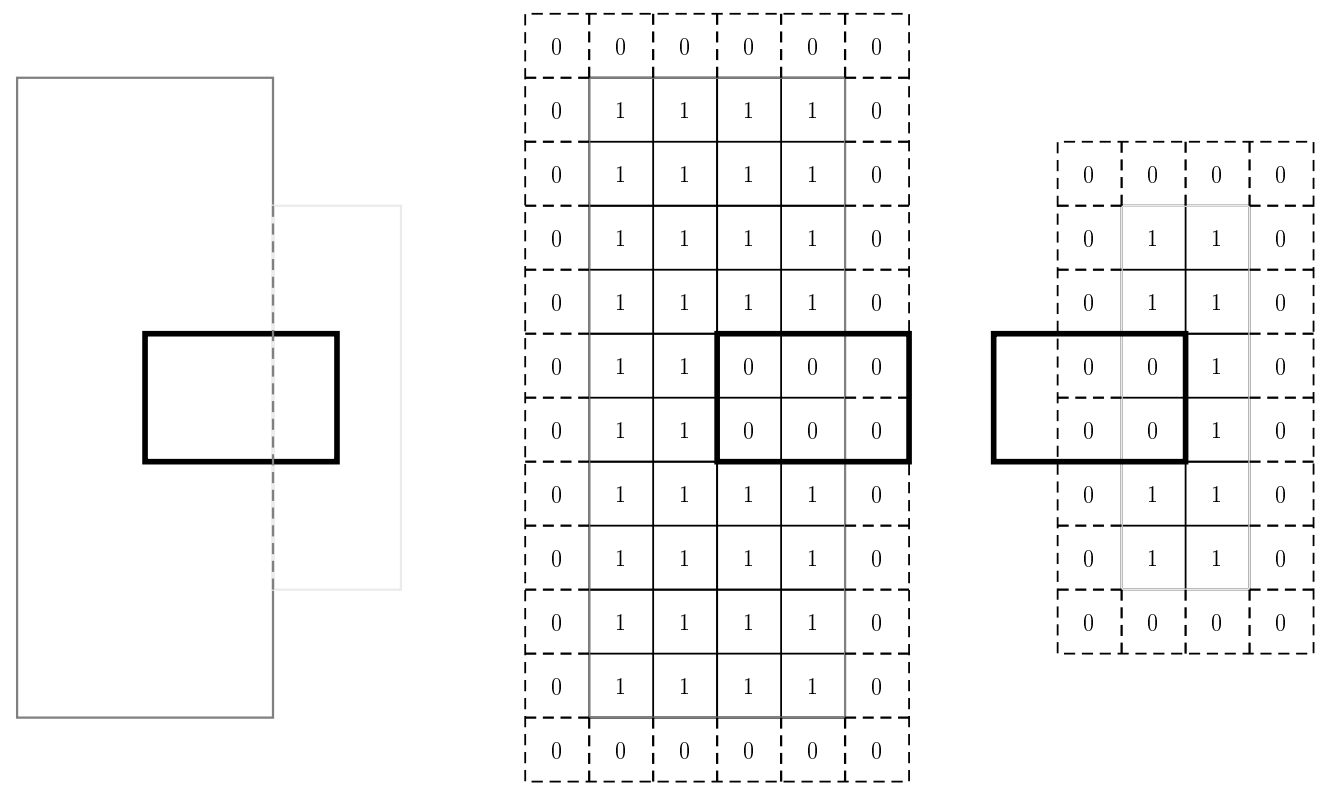

Figura 4.10: Mapa de visibilidade nível base $+1=L_{2}$ no centro da célula.

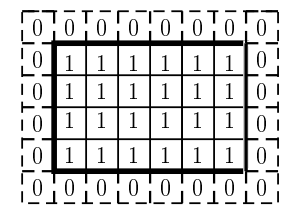

Figura 4.11: Nível base $+2=L_{3}$ no centro da célula.

Os mapas de visibilidade para variáveis definidas em meio de aresta na direção $x$ ou direção $y$, para nossa malha modelo, podem ser vistos no Apêndice B.

Para obtermos uma representação matricial que esteja associada a um conjunto de equações lineares oriundas de uma discretização para uma equação diferencial parcial, é necessário uma enumeração que relacione de forma consistente os valores de cada posição vetorial com uma posição matricial correspondente. Esta enumeração, no caso do AMR, é dada só para as variáveis que devem ser resolvidas, ou seja, nas células (arestas) visíveis, as quais são o nosso real interesse nas discretizações empregadas. Por isso, variáveis que estejam recobertas por níveis mais finos ou que sejam células fantasmas não possuem enumeração.

A enumeração de centros de células (arestas) não é única. Dependendo da maneira como ela é feita, essa enumeração poderá auxiliar a posicionar de forma mais conveniente os centros de células (arestas) que tenham alguma relação especial de vizinhança, como por exemplo, o estêncil da discretização ou de interpolação. Este tipo de preocupação é particularmente importante no contexto de programação paralela, pois se centros de células (arestas) próximos receberem números próximos, eles ocuparão posições próximas na matriz e isto facilitará a comunicação entre processadores diferentes. Tendo em vista que será necessário explorar no futuro diferentes formas de se enumerar centros de células (arestas) para determinar melhores algoritmos, visando ao aumento da eficiência (diminuição de comunicação) no contexto paralelo, mantivemos a enumeração como um dado de entrada. Em um outro contexto igualmente importante, as propriedades da matriz podem ser drasticamente modificadas do ponto de vista de Álgebra Linear Computacional. Enumerações diferentes certamente poderão alterar propriedades como as dadas pelo raio espectral, número de 
condição, autovetores, simetria (maior ou menor), gráfico de esparsidade, etc. Notamos que é possível encontrar na literatura outras enumerações ou ordens de percurso das células computacionais que seguem curvas especiais para a técnica SAMR, como por exemplo as Curvas de Morton [1].

Assim sendo, baseados em uma dada enumeração, formamos a matriz que reflete o estêncil de uma discretização desejada. Nessa matriz, devemos obter o o padrão e a conexão entre as células nos diferentes níveis estabelecidos pelas interpolações em células fantasmas e em células cobertas.

Em nosso caso, trabalhamos com uma enumeração que consideramos a mais "natural" dentro do contexto de malhas compostas, como as definidas pela técnica SAMR. Por exemplo, para os centros de células das células visíveis, a enumeração é feita nível a nível por varredura sequencial de seus respectivos retalhos, respeitando a ordem em que os retalhos aparecem na estrutura de dados que os contêm. Tal ordem, neste instante, não obedece a nenhuma regra pré-estabelecida e é determinada durante o processo de remalhagem. Os retalhos dentro de um nível que são criados primeiro são armazenados primeiro. Dado um retalho, iniciamos a enumeração com o contador incrementado de "um", isto é, com o número seguinte ao último empregado no retalho enumerado anteriormente. As células são varridas da esquerda para a direita, linha a linha, iniciando na célula do canto inferior esquerdo do retalho. Na Figura 4.12, temos a enumeração para variáveis definidas nos centros de células para a malha que tomamos como referência dada na Figura 4.2(b). Observe que seguimos seu respectivo mapa de visibilidade dado nas figuras 4.9, 4.10 e 4.11 .

\begin{tabular}{|c|c|c|c|c|c|c|c|c|}
\hline 24 & 25 & \multicolumn{2}{|c|}{26} & \multicolumn{2}{|c|}{27} & \multicolumn{2}{|c|}{28} & 29 \\
\hline \multirow{2}{*}{20} & \multirow{2}{*}{21} & 72 & 73 & 74 & 75 & \multirow{2}{*}{\multicolumn{2}{|c|}{22}} & \multirow{2}{*}{23} \\
\hline & & 68 & 69 & 70 & 71 & & & \\
\hline \multirow{2}{*}{17} & \multirow{2}{*}{18} & 64 & 65 & 66 & 67 & 38 & 39 & \multirow{2}{*}{19} \\
\hline & & 60 & 61 & 62 & 63 & 36 & 37 & \\
\hline \multirow{2}{*}{14} & \multirow{2}{*}{15} & 58 & 59 & \begin{tabular}{|l|l|}
94 & 95 \\
88 & 89 \\
\end{tabular} & \begin{tabular}{|l|l|}
96 & 97 \\
90 & 91 \\
\end{tabular} & \begin{tabular}{|l|l|}
98 & 99 \\
92 & 93 \\
\end{tabular} & 35 & \multirow{2}{*}{16} \\
\hline & & 56 & 57 & \begin{tabular}{|l|l|}
82 & 83 \\
76 & 77 \\
\end{tabular} & 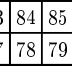 & \begin{tabular}{|l|l|}
86 & 87 \\
\end{tabular} & 34 & \\
\hline \multirow{2}{*}{11} & \multirow{2}{*}{12} & 52 & 53 & 54 & 55 & 32 & 33 & \multirow{2}{*}{13} \\
\hline & & 48 & 49 & 50 & 51 & 30 & 31 & \\
\hline \multirow{2}{*}{7} & \multirow{2}{*}{8} & 44 & 45 & 46 & 47 & \multirow{2}{*}{\multicolumn{2}{|c|}{9}} & \multirow{2}{*}{10} \\
\hline & & 40 & 41 & 42 & 43 & & & \\
\hline 1 & 2 & & & & 4 & 5 & & 6 \\
\hline
\end{tabular}

Figura 4.12: Enumeração no centro da célula.

A enumeração para variáveis definidas em meio de arestas $x$ ou de arestas $y$ é realizada seguindo a mesma ideia de enumeração para variáveis definidas em centros de célula. Enumeramos de forma consecutiva da esquerda para a direita, seguindo a ordem dos retalhos em cada nível e iniciando no nível mais grosso até o mais fino.

Dado que as posições visíveis são as únicas que receberão enumeração, o uso do mapa de visibilidade é muito importante para a enumeração das faces, já que arestas compartilhadas devem ter a mesma enumeração (elas devem definir a mesma linha na matriz e nunca linhas diferentes). No 
Apêndice $\mathrm{C}$, temos um exemplo de enumeração para variáveis definidas no meio de $\operatorname{arestas} x$ e de arestas $y$.

Empregamos a enumeração definida acima em nossa implementação mas, como mencionamos, também poderíamos empregar outras enumerações, dadas por outros algoritmos ou até mesmo fornecidas como dados de entrada. Note entretanto que, qualquer que seja o caso, tais enumerações só poderão ser utilizadas no programa se elas respeitarem o mapa de visibilidade.

\subsubsection{Representação matricial de células fantasmas}

Para obter a representação matricial dos operadores de diferenças empregados em malhas compostas obtidas com a técnica SAMR, iniciamos com o armazenamento do estêncil (e de seus respectivos pesos) que definem valores em células recobertas e fantasmas (isto é, nas células invisíveis). O "caminho de interpolações" seguido para obter as aproximações é dado pelos passos apresentados na Seção 4.1.2. No que segue, a palavra "célula" é usada indistintamente para significar as possíveis posições assumidas pelas variáveis, isto é, ou centro de célula ou centro de aresta (vertical ou horizontal). Eventuais diferenças de tratamento, quando necessárias, serão explicitadas.

Como mencionamos anteriormente, empregamos listas ligadas para obtermos a estrutura matricial que armazena o estêncil de interpolações. É importante lembrarmos também que, em células visíveis, não há nenhuma interpolação e que, portanto, em suas listas ligadas haverá apenas um nó - além da cabeça - o qual armazenará apenas o índice que identifica sua própria célula (seu "Id" de célula, a célula de onde ela parte), e o seu respectivo peso (no caso, "um"). Mostramos um exemplo na Figura 4.13(a).

Após determinarmos as listas ligadas de todas as células visíveis e de todas as células invisíveis, com seus respectivos estênceis de interpolação de células fantasmas e de células recobertas, as listas ligadas poderão conter de um a $m$ nós com pesos $w_{i}$, para $i=1 . . m$, diferentes de zero. Cada $I d_{i}$ de célula relaciona seu respetivo $w_{i}$ com uma célula visível. Na Figura 4.13(b), mostramos a lista ligada relacionada a uma célula invisível da estrutura matricial que armazena as interpolações de células fantasmas.

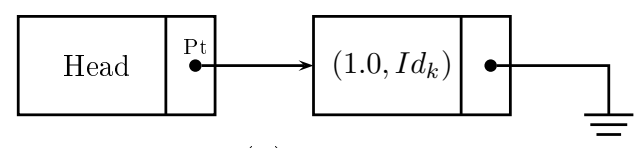

(a)

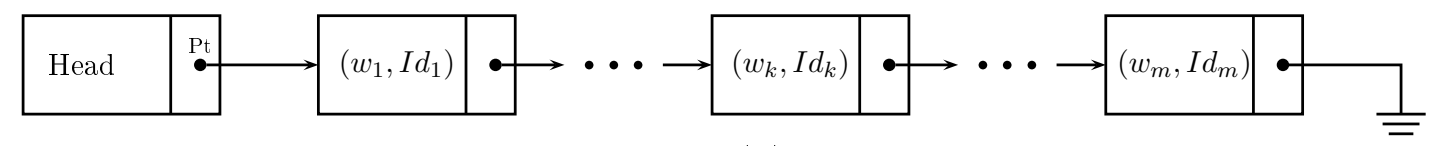

(b)

Figura 4.13: Representação de uma lista ligada associada a uma célula (a) visível e (b) invisível para um estêncil relacionado com $m$ células computacionais

Com base na enumeração, formamos a matriz que reflete o estêncil da discretização desejada. Esta matriz deve obter para todos os níveis da malha o comportamento que as interpolações em células fantasmas e células recobertas teria. Para conseguir isso, é necessário definir uma operação de listas ligadas que realize operações da forma $A+w\left(w_{1} B_{1}+w_{2} B_{2}+\cdots+w_{n} B_{n}\right)=A+w *$ $w_{1} B_{1}+w * w_{2} B_{2}+\cdots+w * w_{n} B_{n}$ e depois agrupe os termos de mesma classe. 
Para duas listas ligadas $A$ e $B$ da estrutura de dados definida, a operação merge $(A, B, w)$ consiste em inserir na lista A todos os elementos da lista B para os quais cada um dos pesos $w_{k}$ da lista é multiplicado por $w$. A inserção na lista ligada foi definida como uma inserção ordenada pela chave $I d$ de cada nó e, em caso de repetição, os pesos de cada lista são somados [89].

O algoritmo implementado para definir a estrutura matricial de interpolações das células fantasmas, isto é, armazenar os valores dos pesos que determinam cada estêncil de interpolação, reproduz exatamente as chamadas para a interpolação das variáveis de células fantasmas da estrutura na implementação AMR2D. Os passos são os seguintes:

Passo 1. Interpolação cúbica em uma camada de células recobertas por retalhos mais finos;

Passo 2. Interpolação fina-grossa;

Passo 3. Ajuste de valores entre malhas irmãs;

Passo 4. Aproximação pelas condições de contorno.

A definição de células fantasmas dada acima é diferente da que foi dada na Seção 4.1.2: nela, não incluímos o cálculo da média das variáveis recobertas, dado que a matriz para a discretização não precisa da aproximação nelas para a obtenção das variáveis em células visíveis. A média aritmética entre variáveis de diferentes níveis é usada para definir as variáveis para as operações retalho-aretalho, o que não é o caso da forma matricial.

Para o Passo 1, na forma matricial, a aproximação de uma célula recoberta com a extrapolaçãointerpolação cúbica vai armazenar o estêncil da interpolação que vai ser dado por uma das combinações possíveis dadas nas figuras 4.14(a)-(d).

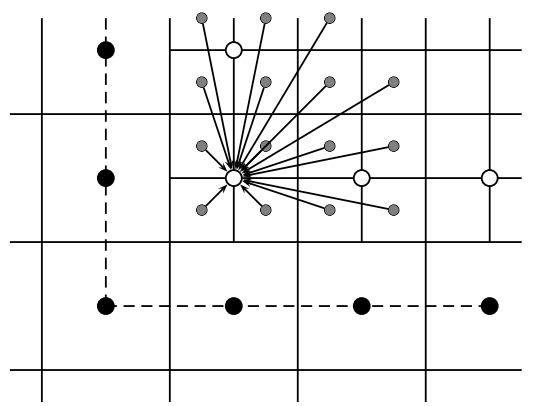

(a)

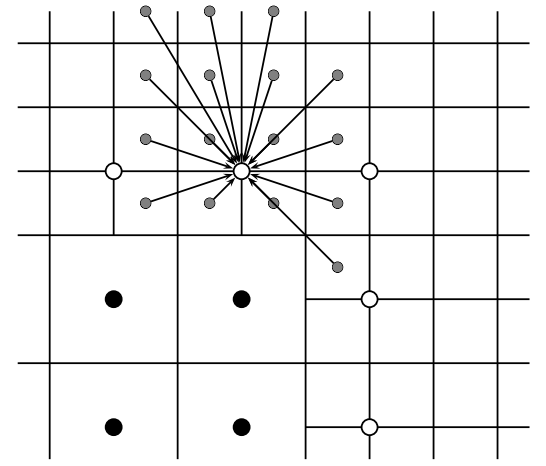

(c)

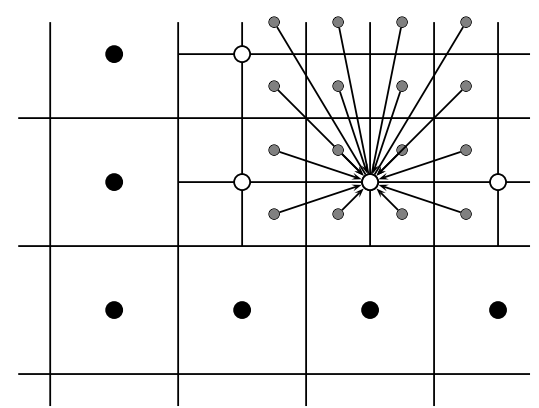

(b)

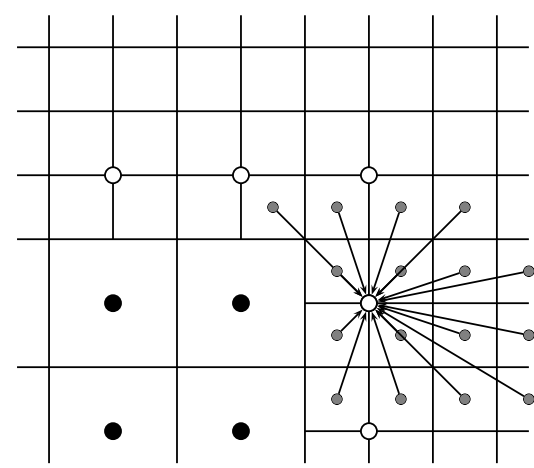

(d)

Figura 4.14: Estêncil para interpolação cúbica em diferentes posições.

Na forma matricial, ilustramos o Passo 2 na Figura 4.15, a qual mostra como é armazenado o 
estêncil da interpolação de uma célula fantasma definida na interface entre um bloco de um nível grosso e o consecutivo mais fino.
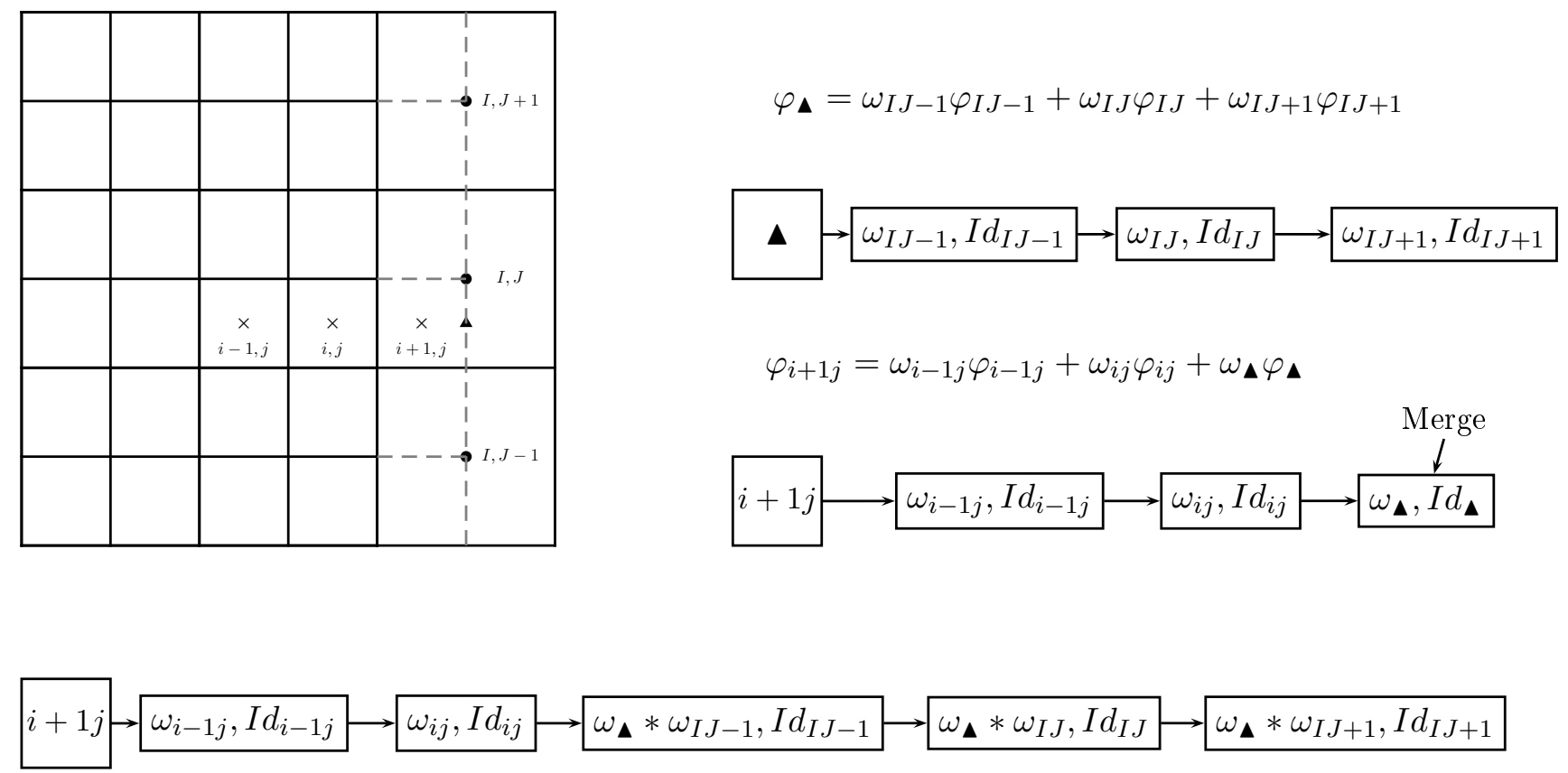

Figura 4.15: Interpolação malha fina-grossa na célula $(i+1, j)$.

Mostramos na Figura 4.16(a)-(b) o estêncil da interpolação quadrática na interface fina-grossa no caso da interseção de dois retalhos diferentes do mesmo nível onde é formado uma T-junctions. Aqui, podemos ver como o estêncil traz informação dos dois retalhos e a grande quantidade de células finas que estão envolvidas na interpolação. Se algumas das células do nível fino que estão relacionadas com este estêncil vêm de uma outra interpolação, elas estarão relacionadas com outro estêncil e teríamos mais células envolvidas na interpolação da Figura 4.16(b).

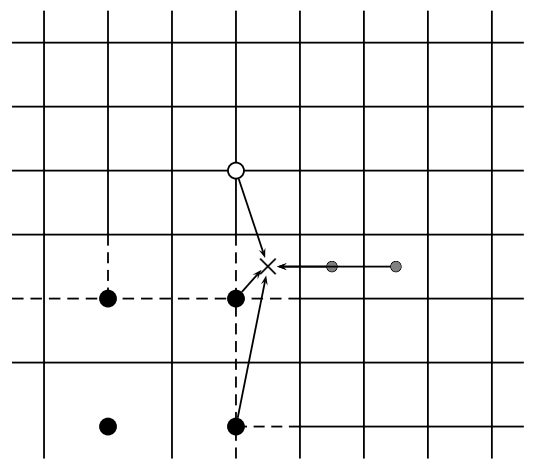

(a)

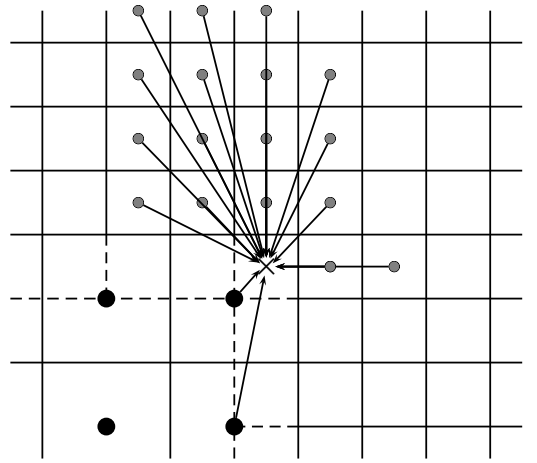

(b)

Figura 4.16: Estêncil da interpolação fina grossa em T-Junctions.

Ao terminar a etapa de preparação da lista ligada relacionada com cada célula invisível, por meio de interpolações que garantam a correta comunicação entre retalhos, cada uma destas listas terá no mínimo uma célula e no máximo a quantidade de células visíveis da malha, sendo que o último caso é improvável.

Dizemos que uma malha é "trivial" se sua malha base está constituída por uma única célula. Em 
geral, como trabalharemos com malhas não triviais, a quantidade de células é estritamente menor do que a quantidade de células visíveis na malha.

Com esta informação podemos encontrar, por meio do produto de uma matriz por um vetor, o valor resultante para cada chamada da interpolação no algoritmo usual no SAMR que define os valores nas células fantasmas. Esta matriz é a que armazena o estêncil de cada um dos passos para a aproximação de células fantasmas, de células recobertas e de células visíveis, como ilustrado na Figura 4.13(a).

Assim, o cálculo de valores em células invisíveis $\varphi_{I V}$ de uma variável $\varphi$ definida em uma malha composta é dado em forma vetorial por uma enumeração e pode ser feito com o produto de uma matriz $A_{G}$ contendo o estêncil de células fantasmas e o vetor correspondente $\varphi_{V}$ de células visíveis somado com um vetor $\varphi_{c c}$ dependente das condições de contorno. Dessa maneira,

$$
\varphi_{I V}=A_{G} \varphi_{V}+\varphi_{c c}
$$

Para definir as matrizes relacionadas com os operadores discretos é necessário que usemos a definição da estrutura matricial para as células fantasmas para, assim , conhecer o estêncil das aproximações nas células fantasmas.

\subsubsection{Representação matricial das discretizações}

A matriz que representa alguma discretização tem uma dimensão que depende da quantidade de células visíveis, pois é nelas que estão definidas as equações. Se o sistema é para uma variável então a dimensão da matriz é o número de células visíveis da variável. Se a matriz é para resolver sistema linear acoplado com duas variáveis, então a dimensão da matriz é a soma da quantidade de células visíveis para cada variável.

A matriz que tem a discretização é definida seguindo a mesma estrutura de lista ligada para cada célula computacional. Quando a matriz já esta definida na estrutura de dados, pode ser passada para uma biblioteca externa, como o PETSc, seguindo apropriadamente a maneira de se fazer a interface com cada biblioteca [11].

Antes de definir a discretização, deve ser criada a estrutura matricial de células fantasmas segundo a posição da variável na célula. Note que só é necessária uma estrutura matricial de células fantasmas para cada tipo de variável. Variáveis nas mesmas posições (em centros de célula ou em meios de arestas), independentemente de sua quantidade, compartilham a mesma estrutura matricial. O estêncil de células fantasmas e a operação merge de células definem a matriz de discretização. Dessa forma, não precisamos ter nenhum cuidado com a técnica SAMR e com a inserção de elementos da matriz respeitando as regras da lista. A definição dos pesos da matriz é feita de forma muito similar a do caso uniforme [88].

Por exemplo, o estêncil de cinco pontos para a aproximação do operador laplaciano, para uma variável definida no centro, é dado por

$$
\begin{aligned}
\mathbf{L} \varphi & =\frac{\varphi_{i+1 j}-2 \varphi_{i j}+\varphi_{i-1 j}}{\Delta x^{2}}+\frac{\varphi_{i j+1}-2 \varphi_{i j}+\varphi_{i j-1}}{\Delta y^{2}} \\
& =\frac{1}{\Delta x^{2}} \varphi_{i-1 j}+\frac{1}{\Delta y^{2}} \varphi_{i j-1}-2\left(\frac{1}{\Delta x^{2}}+\frac{1}{\Delta y^{2}}\right) \varphi_{i j}+\frac{1}{\Delta y^{2}} \varphi_{i j+1}+\frac{1}{\Delta x^{2}} \varphi_{i+1 j},
\end{aligned}
$$

onde cada termo deste estêncil relaciona a enumeração de cada célula com seu respectivo peso. 
Neste caso, o valor na posição da célula $(i, j)$ com número de linha da matriz dado por $I d_{i, j}$. Em função disto, criamos a lista ligada $l_{D}$ que armazenará o estêncil da discretização, como segue.

1. Da célula $(i, j)$ usamos a lista ligada $l_{G_{i j}}$ a qual corresponde à interpolação das células fantasmas. Nesta posição, o peso a ser inserido é $w_{i, j}=-2\left(\frac{1}{\Delta x^{2}}+\frac{1}{\Delta y^{2}}\right)$ e faz merge $\left(l_{D}, l_{G_{i j}}, w_{i j}\right)$ onde a lista $l_{D}$ estava inicialmente vazia.

2. Da célula $(i-1, j)$ usamos a lista ligada $l_{G_{i-1 j}}$, a qual corresponde à interpolação das células fantasmas, e o peso a ser inserido é $w_{i-1, j}=\frac{1}{\Delta x^{2}}$ e faz merge $\left(l_{D}, l_{G_{i-1 j}}, w_{i-1 j}\right)$ onde a lista $l_{D}$ tinha o estêncil inserido no passo anterior.

3. Da célula $(i+1, j)$ usamos a lista ligada $l_{G_{i+1 j}}$, a qual corresponde à interpolação das células fantasmas e o peso a ser inserido é $w_{i+1, j}=\frac{1}{\Delta x^{2}}$ e faz merge $\left(l_{D}, l_{G_{i+1 j}}, w_{i+1 j}\right)$.

4. Da célula $(i, j+1)$ usamos a lista ligada $l_{G_{i j+1}}$, a qual corresponde à interpolação das células fantasmas e o peso a ser inserido é $w_{i, j+1}=\frac{1}{\Delta y^{2}}$ e faz merge $\left(l_{D}, l_{G_{i j+1}}, w_{i j+1}\right)$.

5. Da célula $(i, j-1)$ usamos a lista ligada $l_{G_{i j-1}}$, a qual corresponde à interpolação das células fantasmas e o peso a ser inserido $w_{i, j-1}=\frac{1}{\Delta y^{2}}$ e faz merge $\left(l_{D}, l_{G_{i j-1}}, w_{i j-1}\right)$.

A inserção dos elementos da matriz é natural ao se usar a rotina merge definida de forma particular para as listas. A lista ligada da estrutura de células fantasmas na posição $(i, j)$ sempre é de um nó visível. Por outro lado, não temos certeza qual das outras células $(i-1, j),(i+1, j)$, $(i, j+1)$ e $(i, j-1)$ corresponde a um nó visível, mas isto não preocupa porque, se algum deles não é visível, a lista $l_{G}$ na sua posição já tem o estêncil da interpolação de células fantasma que lhe corresponde e merge insere cada um destes na lista ligada $l_{D}$ multiplicando cada peso pelo correspondente peso da discretização.

A matriz tem dimensão do número de células visíveis e devemos ter um mapa que relaciona cada posição das variáveis visíveis com seu índice de enumeração e com isto evitamos realizar buscas.

Para definir uma outra matriz de discretização, temos que seguir os mesmos passos: criar a estrutura matricial que armazena em cada célula uma lista ligada associada a um estêncil e depois, realizar em cada linha da matriz e para cada $(i, j)$ a chamada de merge com a respectiva lista ligada de células fantasmas e o peso da interpolação.

Esta matriz é depois passada para o PETSc, que resolve o sistema segundo as opções que nós desejamos. Com pré-condicionador ou sem, com paralelismo ou não e com o solver selecionado.

\subsection{SAMR Método de Uzawa com BiCG-Stab (versão matricial)}

Finalizamos o presente capítulo mostrando como fica o algoritmo do Método de Uzawa baseado no Método BiCG-Stab empregando, para as velocidades, a forma matricial. Seguimos aqui a apresentação dada na Seção 3.5 do capítulo anterior e as discretizações na malha composta dadas na Seção 4.1.3. A solução do sistema matricial para as velocidades só retorna para a malha composta as aproximações para as arestas visíveis. Devido a isso, para continuar as operações retalho-a-retalho, nas quais se baseiam o Método BiCG-Stab, é preciso que a variável esteja bem definida na estrutura de dados do SAMR. Neste contexto, o algoritmo para a solução da equação estacionária de Stokes (2.44) na malha composta é dado como segue abaixo. 
Algoritmo 2 (SAMR Método de Uzawa com BiCG-Stab retalho-a-retalho).

$1: q=\mathbf{f}+\mathbf{G} p^{(0)}$

2: $\mathbf{L u}=q$
Gradiente na malha composta.

Solução matricial, onde $\mathbf{L}$ define o estêncil da discretização do operador Laplaciano na malha composta.

3: correct_DefineVar $(\mathbf{u})$

$4: r=-\mathbf{D} \cdot \mathbf{u}^{(0)}$

Divergente na malha composta.

5: calc_Ghost(r)

Dirichlet homogênea.

$6: r_{t}=r$

7: while (não converge)

8: $\quad \gamma_{1}=<r, r_{t}>_{l}$

9: $\quad$ if $\left(\gamma_{1}=0\right) \mathbf{O}$ metodo falha

10: $\quad$ if $(\mathrm{i}=1)$

11: $\quad \mathrm{q}=\mathrm{r}$

12: else

13: $\quad \beta=\left(\gamma_{1} / \gamma_{2}\right)(\alpha / \omega)$

14: $\quad q=r+\beta(q-\omega w)$

15: $\quad$ end if

16: $\quad \mathbf{L z}_{1}=\mathbf{G} q$

17: $\quad$ correct_DefineVar $\left(z_{1}\right)$

18: $\quad w=\mathbf{D} \cdot \mathbf{z}_{1}$

19: $\quad \alpha=\gamma_{1} /<r_{t}, w>_{l}$

20: $s=r-\alpha w$

21: calc_Ghost $(s) \quad$ Dirichlet homogênea

22: $\quad \mathbf{L z}_{2}=\mathbf{G} s$

23: $\quad$ correct_DefineVar $\left(z_{2}\right)$

24: $\quad h=\mathbf{D} \cdot \mathbf{z}_{2}$

25: $\quad \omega=<h, s>_{l} /<h, h>_{l}$

26: $\quad p^{(i)}=p^{(i-1)}+\alpha q+\omega s$

27: $\quad \mathbf{u}^{(i)}=\mathbf{u}^{(i-1)}+\alpha \mathbf{z}_{1}+\omega \mathbf{z}_{2}$

28: $\quad r=s-\omega h$

29: calc_Ghost $(r) \quad$ Dirichlet homogênea

30: $\quad \gamma_{2}=\gamma_{1}$

31: $\quad$ if $(\omega=0) \mathbf{O}$ método falha

32: Testar a convergência

33: end while

34: calc_Ghost $\left(p^{(1)}\right)$

35: calc_Ghost $\left(u^{(1)}\right)$ 


\section{Capítulo 5}

\section{Resultados numéricos}

Neste Capítulo, apresentamos resultados numéricos com o intuito de verificar os métodos e as técnicas introduzidas em capítulos precedentes. Este se inicia com a Seção 5.1, na qual descrevemos brevemente como são calculadas as normas e como a análise de convergência numérica será efetuada nas seções subsequentes. Na Seção 5.2, apresentamos resultados relativos à verificação do cálculo de valores das células fantasmas empregando a forma matricial. A verificação do uso da estrutura matricial para representar discretizações na técnica SAMR, é feita na Seção 5.3 por meio de três problemas-modelo, escolhidos especificamente e que podem ser usados no futuro na solução de equações de Navier-Stokes em forma matricial com o Método da Projeção e também na a solução da equação elíptica nas arestas que aparece na solução da equação de Stokes estacionária via o Método Uzawa BiCG-Stab. O tensor viscoelástico modelado pelo Oldroyd-B ou pelo FENE-P é verificado na Seção 5.4. A solução das equações de Navier-Stokes para escoamentos incompressíveis viscoelásticos é verificada com solução manufatura na Seção 5.5. Por último, na Seção 5.6, apresentamos resultados numéricos para a solução da equação de Stokes estacionária usando o Método de Uzawa, com solução manufaturada.

\subsection{Normas e análise da convergência numérica em malhas uni- formes e em malhas compostas}

Antes de apresentarmos qualquer resultado numérico, é necessário descrevermos as normas com as quais avaliaremos os erros tanto em malhas uniformes quanto em malhas compostas. Além disso, precisamos também descrever como será efetuada a análise da convergência numérica.

Em malhas descolocadas como as do tipo Marker-And-cell (MAC), quer sejam uniformes quer sejam compostas, as localizações das variáveis escalares e vetoriais assumem três diferentes discretizações do domínio físico $\Omega: \Omega_{p}^{h}$, onde posicionamos todas as variáveis escalares, é a união de todos os centros de células; $\Omega_{u}^{h}$, onde posicionamos a primeira componente de todas as variáveis vetoriais, é a união dos pontos médios de todas as arestas verticais; e $\Omega_{v}^{h}$, onde posicionamos a segunda componente de todas as variáveis vetoriais, é a união dos pontos médios de todas as arestas horizontais. Com isto em mente, definimos as normas $\|\cdot\|_{1},\|\cdot\|_{2}$ e $\|\cdot\|_{\infty}$ de uma variável escalar 
$\phi$ definida em $\Omega_{p}^{h}$ como

$$
\begin{aligned}
\|\phi\|_{q} & =\left(\sum_{k \in \Omega_{p}^{h}}\left|\phi_{k}\right|^{q} h_{k}^{2}\right)^{1 / q}, \quad q=1,2, \\
\|\phi\|_{\infty} & =\max _{k \in \Omega_{p}^{h}}\left\{\left|\phi_{k}\right|\right\},
\end{aligned}
$$

onde $\phi_{k}$ é o valor assumido por $\phi$ no centro da $k$-ésima célula cujas arestas medem $\Delta x=\Delta y=h_{k}$ (a igualdade é apenas para simplificar a apresentação e não representa nenhuma limitação das definições).

Se definirmos $\psi=\left(\psi_{1}, \psi_{2}\right)$, uma variável vetorial, então $\psi_{1}$ estará posicionada em $\Omega_{u}^{h}$ e $\psi_{2}$ em $\Omega_{v}^{h} \mathrm{e}$

$$
\begin{aligned}
\|\psi\|_{q} & =\left(\sum_{i \in \Omega_{u}^{h}}\left|\psi_{1 i}\right|^{q} a_{i}+\sum_{j \in \Omega_{v}^{h}}\left|\psi_{2 j}\right|^{q} b_{j}\right)^{1 / q}, \quad q=1,2, \\
\|\psi\|_{\infty} & =\max \left\{\max _{i \in \Omega_{u}^{h}}\left\{\left|\psi_{1 i}\right|\right\}, \max _{j \in \Omega_{v}^{h}}\left\{\left|\psi_{2 j}\right|\right\}\right\},
\end{aligned}
$$

onde

$$
a_{i}= \begin{cases}H^{2} & , \text { nas arestas grossas } \\ H^{2} / r^{2} & , \text { nas arestas finas } \\ H^{2}(r+1) /\left(2 r^{2}\right) & , \text { nas arestas de interface fina-grossa }\end{cases}
$$

sendo $r$ a razão de refinamento entre os níveis $l$ e $l+1$ e $H$, o espaçamento da malha no nível $l$. Definimos os pesos $b_{j}$ de maneira similar.

O tensor definido para o armazenamento do tensor viscoelástico tem todas as suas componentes definidas nos centros de células, onde usamos as normas $\|\cdot\|_{2}$ e $\|\cdot\|_{\infty}$ definidas para variáveis centradas nas comparações realizadas com cada uma das componentes tensoriais.

Para efetuarmos a análise de convergência numérica, assumiremos que a solução numérica obtida pelo método possui uma expansão assintótica em potências do espaçamento da malha. Em uma malha uniforme, por exemplo, uma variável escalar $\phi$ tem solução numérica $\phi(\mathbf{x}, t, h)$ a qual, por hipótese, pode ser escrita na forma

$$
\phi(\mathbf{x}, t, h)=\phi_{e}(\mathbf{x}, t)+E_{q}(\mathbf{x}, t) h^{q}+E_{q+1}(\mathbf{x}, t) h^{q+1}+\cdots
$$

para todos os espaçamentos $h$ suficientemente pequenos, onde $\phi_{e}(\mathbf{x}, t)$ é a solução exata e as funções que definem os coeficientes $E_{i}, i=q, q+1, \ldots$, independem de $h$.

Em diversos dos problemas-modelo que empregaremos nas seções a seguir, a solução exata será conhecida a priori. Tal abordagem é comumente denominada como verificação por solução manufaturada [95] e, hoje, é o padrão aceito na comprovação da correta implementação de metodologias numéricas. Para nossos problemas-modelo com solução conhecida, depois de calcularmos um valor aproximado $\phi(\mathbf{x}, t, h)$ para um dado espaçamento $h$, calculamos para o mesmo ponto da malha $(\mathbf{x}, t)$, mas desta vez com espaçamento $h / 2$, a aproximação $\phi(\mathbf{x}, t, h / 2)$. Assim, em primeira aproximação 
teremos

$$
\begin{aligned}
\phi(\mathbf{x}, t, h) & \approx \phi_{e}(\mathbf{x}, t)+E_{q}(\mathbf{x}, t) h^{q} \\
\phi\left(\mathbf{x}, t, \frac{h}{2}\right) & \approx \phi_{e}(\mathbf{x}, t)+E_{q}(\mathbf{x}, t) \frac{h^{q}}{2^{q}}
\end{aligned}
$$

o que nos fornece a aproximação para a razão de convergência

$$
\frac{\left\|\phi(\mathbf{x}, t, h)-\phi_{e}(\mathbf{x}, t)\right\|}{\left\|\phi\left(\mathbf{x}, t, \frac{h}{2}\right)-\phi_{e}(\mathbf{x}, t)\right\|} \approx 2^{q}
$$

onde $\|\cdot\|$ representa a norma (5.1) ou (5.2). Observe que as razões de convergência 2, 4, 8,.., significam que o método numérico tem ordem de precisão $q=1,2,3, \ldots$

Empregaremos a estimativa da razão de convergência (5.5) tanto em malhas uniformes quanto em malhas compostas. Quando necessário, faremos observações suplementares sobre o cálculo de normas e sobre a análise de convergência numérica empregando refinamento de malha.

\subsection{Verificação do cálculo matricial de valores fantasmas}

Comumente, os sistemas lineares resultantes do processo de discretização das equações diferenciais parciais em malhas com refinamento adaptativo são resolvidos por intermédio de métodos do tipo multinível-multigrid [65, 85, 102]. Tipicamente, a implementação desses métodos de solução em malhas compostas depende de laços aninhados sobre a hierarquia de níveis de refinamento e sobre os retalhos de malha que cada um destes níveis contém. Frequentemente, durante a aplicação do método precisamos chamar as rotinas de cálculo de valores fantasmas. A implementação computacional de tais rotinas em nossos códigos domésticos, por simplicidade e por eficiência de implementação, empregam as mesmas posições de memória para efetuar uma diversidade grande de cálculos auxiliares com objetivos distintos ao longo do processo de interpolação.

Em um primeiro momento, procurar estratégias de solução baseadas no Método Multigrid para as equações estacionárias de Stokes em malha composta pareceu ser bastante natural. Entretanto, percebemos, tal metodologia é sutil e de complexa implementação neste caso - até mesmo em malhas uniformes. Uma das razões é o surgimento de zeros na diagonal principal da matriz do sistema linear, o que impede o uso de relaxadores tradicionais como o de Jacobi e o de Gauss-Seidel (saddle point problem $)[8,21,72,76,114,115]$. Outras propostas de relaxação se fazem necessárias e sua tradução para malhas compostas não é clara. Como alternativa às técnicas multigrid, decidimos investir no desenvolvimento de uma versão adaptativa do Método de Uzawa [4, 7, 56, 57, 117].

Embora bastante naturais no contexto da metodologia multinível-multigrid, as rotinas de cálculos de valores fantasmas, ao dependerem tão fortemente do uso e do descarte contínuo de valores auxiliares para fins diferentes - sempre fazendo uso das mesmas posições de memória - tornou extremamente complexa a tarefa de desenvolvermos e implementarmos outras estratégias de solução de sistemas lineares sobre esta estrutura. Como exemplo, podemos citar nossa tentativa (num primeiro momento frustrada) de implementar sobre a estrutura de malha composta o Método dos Gradientes Bi-conjugados Estabilizado (BiCG-Stab) empregando os algoritmos e as rotinas inicialmente existentes de cálculo de valores fantasmas. Como discutimos em capítulos anteriores, o Método de Uzawa tem no BiCG-Stab uma de suas principais "máquinas" de cálculo e que deve ser empregado 
mesmo no contexto de malhas SAMR.

Além da dificuldade de implementação de novas estratégias de solução, é bem conhecida a dificuldade em se obter a convergência de métodos multigrid em problemas nos quais, por exemplo, há fortes anisotropias de espaçamento nas malhas espaciais ou quando há saltos "grandes" de propriedades materiais. Este é certamente o caso da massa específica em escoamentos bifásicos contendo gotas de água em ar (bolhas de ar em água). A simulação em malha composta de tais casos de interesse tem recebido contínua atenção dos grupos em Dinâmica de Fluidos Computacional do IME-USP e da FEMEC-UFU em muitas de suas linhas de pesquisa. Ainda hoje, se faz presente a necessidade de alternativas robustas e eficazes nestas situações onde potencialmente encontramos problemas na convergência dos métodos multinível-multigrid.

Considerando as dificuldades mencionadas acima, tornou-se evidente a necessidade de investigarmos uma nova abordagem, de propormos uma inovaçâa de paradigma: a obtenção de uma representação matricial explícita dos sistemas lineares oriundos das discretizações em malha composta. Uma vantagem indiscutível que traz tal representação matricial é a de permitir o uso de bibliotecas numéricas gratuitas como, por exemplo, Hypre ${ }^{1}$, Trilinos ${ }^{2}$, KINSol e o PETSc, muitas reconhecidas no meio acadêmico por sua robustez e eficiência. Via de regra, elas têm implementada uma incrível variedade de métodos numéricos, inclusive em suas versões paralelas. Assim sendo, o investimento de esforço neste sentido é muito bem recompensado: não só pela conquista de novas possibilidades de se resolver os sistemas lineares mas também pelo ganho em eficiência/velocidade na paralelização.

A obtenção da matriz esparsa que representa o sistema linear oriundo de uma discretização em malha composta é baseada nos mesmos padrões de interpolação definidos no Capítulo 4. Não há, na verdade, razões para alterarmos os interpolantes - apenas para empregá-los de outra maneira. Verificaremos a correta implementação das estruturas matriciais usando no presente trabalho a biblioteca PETSc. Tal biblioteca, desenvolvida pelo Laboratório Nacional de Argonne (ANL) nos Estados Unidos, apresenta opções ao paralelismo compartilhado e distribuído e conta com um grupo de apoio e listas de discussão bastante ativos.

A biblioteca PETSc tem uma grande variedade de ferramentas para problemas de grande porte. Inclui Krylov solvers, pré-condicionadores, opções para solução de problemas não lineares, solução de problemas dependentes do tempo, multigrid algébrico, entre outros. Muitas destas ferramentas têm versão paralela. Mais informação sobre o PETSc podem ser encontradas em [10, 11, 12].

A literatura que discorre sobre alguma metodologia de obtenção da representação matricial explícita em malhas compostas SAMR, como as usadas no presente trabalho é pouca. O que há é bastante difícil de se localizar [41, 42, 53, 83]. Em um primeiro instante na história do desenvolvimento de técnicas de discretização sobre tais malhas, passou desapercebida a importância e a utilidade de se ter disponível tal representação. Certamente, dentre nossos colaboradores, esta é a primeira vez que se emprega matrizes esparsas para armazenar o estêncil de uma discretização em malha composta. Bibliotecas como SAMRAI e Chombo [3, 33, 79, 109], largamente difundidas e utilizadas para resolver equações diferencias parciais em malhas adaptativas do tipo SAMR, não empregam a representação matricial como comentamos no Capítulo 4.

A definição dos problemas-modelo empregados na verificação da correta implementação das

\footnotetext{
${ }^{1}$ http://acts.nersc.gov/hypre

${ }^{2}$ http://trilinos.sandia.gov
} 
estruturas matriciais foi guiada pelas necessidades presentes durante o processo de resolução das equações de Stokes, via Método de Uzawa, e de Navier-Stokes, via Método da Projeção de ChorinTemam. Neste contexto, propusemos duas classes de problemas-modelo: uma para equações elípticas, para variáveis definidas em centros de células e em meios de arestas em aplicações envolvendo escoamentos transientes e estacionários, e uma outra para equações parabólicas, oriundas durante a aplicação de métodos de projeção para escoamentos transientes. No Método de Uzawa, temos que resolver equações elípticas para determinar as componentes das velocidades nos meios de arestas e, no Método da Projeção de Chorin-Temam, para determinar a correção da pressão nos centros de células.

Identificamos como sendo um ponto crucial a tradução da determinação de valores fantasmas da forma convencional no SAMR (i.e. por laços aninhados nos níveis de refinamento e nos retalhos de cada nível) para a forma matricial. Uma vez que se saiba como determinar matricialmente os valores em células fantasmas, não existe nenhuma dificuldade adicional para obtermos explicitamente a matriz que contém a discretização da equação diferencial parcial em estudo.

\subsubsection{Resultados do cálculo matricial de valores fantasmas}

Para verificar se a matriz que armazena o estêncil da interpolação de células fantasmas no SAMR está correta, comparamos os valores das células fantasmas obtidos da maneira convencional no SAMR (operações nível-a-nível e retalho-a-retalho) com aqueles obtidos por um produto matrizvetor. A função que usamos para este teste é

$$
\varphi(x, y)=\frac{\cos (2 \pi x) \sin (2 \pi y)}{2 \pi}
$$

e combinamos de condições de contorno periódicas, de Neumann e de Dirichlet.

Inicialmente, valores exatos $\varphi_{e}$ são armazenados em todas as células computacionais de cada retalho, incluindo as fantasmas, e no vetor empregado na abordagem matricial. Adotamos este procedimento para a malha composta com três níveis de refinamento, ilustrada na Figura 5.1, e para uma variável $\varphi$ definida no centro de célula: $\varphi_{M a t V e c}$ é o valor obtido ao ser usado o produto matriz-vetor e $\varphi_{S A M R}$ é o valor obtido com a implementação nível-a-nível e retalho-a-retalho. A verificação com este teste também foi realizada para variáveis definidas nas arestas verticais e nas horizontais, com diferentes condições de contorno e, como esperado, obtivemos erros na ordem do "zero de máquina" em dupla precisão.

Desejamos ver a diferença entre o cálculo de valores em células fantasmas realizado por meio da estrutura matricial que armazena o estêncil na obtenção de células fantasmas e pelo cálculo usual retalho-a-retalho Calc_ghost. Lembramos que o cálculo dos valores em células fantasmas segue os passos descritos no Capítulo 4, na Seção 4.1.2, para Calc_ghost e na Seção 4.2.2 para o cálculo com a estrutura matricial.

Obtivemos para uma variável definida no centro de célula (e.g. correção de pressão) a aproximação de valores nas células fantasmas para condição de contorno de Dirichlet mostrados na Tabela 5.1, para Neumann na Tabela 5.2 e condição periódica na Tabela 5.3. Note que tanto a aproximação com o produto matricial matriz-vetor quanto o cálculo usual nível-a-nível, retalho-a-retalho, aproximam os valores nas células fantasmas da mesma forma.

Em cada uma das tabelas 5.1-5.3, temos a ordem de convergência com a norma infinito dos inter- 


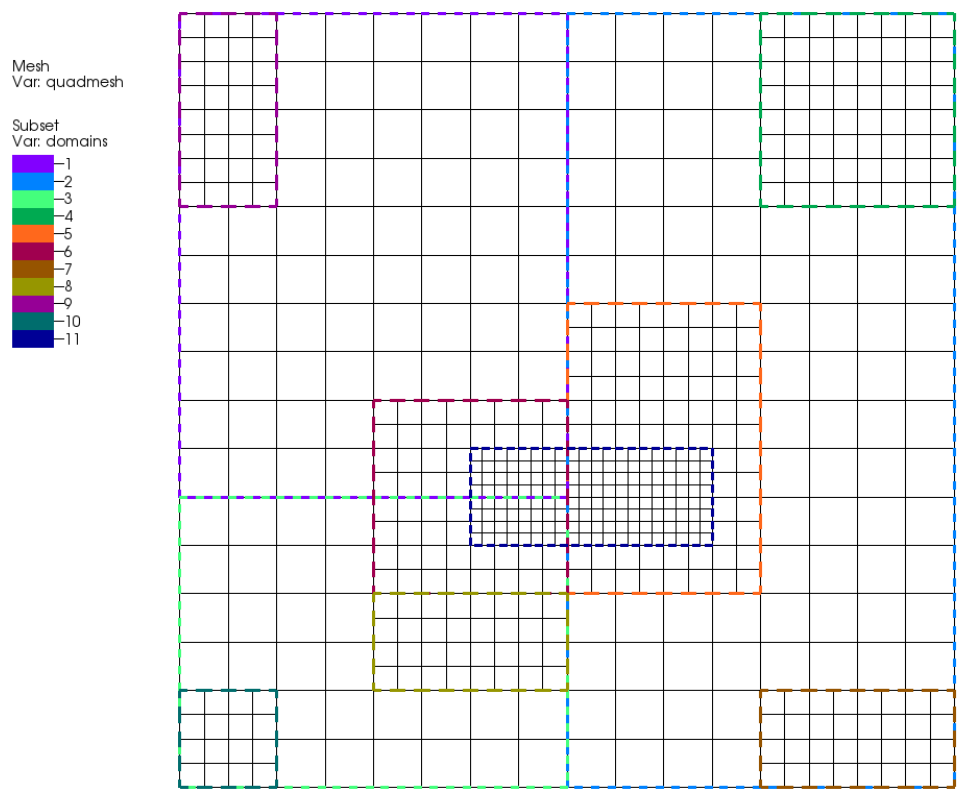

Figura 5.1: Malha composta com três níveis de refinamento e sua divisão em retalhos.

\begin{tabular}{cccccc}
\hline $\mathrm{n}$ & $\mathrm{L}$ & $\left|\varphi_{e}-\varphi_{\text {MatVec }}\right|_{\infty}$ & $r_{\infty}$ & $\left|\varphi_{e}-\varphi_{\text {SAMR }}\right|_{\infty}$ & $r_{\infty}$ \\
\hline 4 & 3 & $5.9987 \mathrm{E}-03$ & - & $5.9987 \mathrm{E}-03$ & - \\
5 & 3 & $1.5254 \mathrm{E}-03$ & 3.9326 & $1.5254 \mathrm{E}-03$ & 3.9326 \\
6 & 3 & $3.8296 \mathrm{E}-04$ & 3.9831 & $3.8296 \mathrm{E}-04$ & 3.9831 \\
7 & 3 & $9.5840 \mathrm{E}-05$ & 3.9958 & $9.5840 \mathrm{E}-05$ & 3.9958 \\
\hline
\end{tabular}

Tabela 5.1: Erro e ordem de convergência no cálculo dos valores das células fantasmas empregando o produto matriz-vetor e o cálculo usual nível-a-nível, retalho-a-retalho. Condição de contorno de Dirichlet.

\begin{tabular}{cccccc}
\hline $\mathrm{n}$ & $\mathrm{L}$ & $\left|\varphi_{e}-\varphi_{\text {MatVec }}\right|_{\infty}$ & $r_{\infty}$ & $\left|\varphi_{e}-\varphi_{\text {SAMR }}\right|_{\infty}$ & $r_{\infty}$ \\
\hline 4 & 3 & $6.0911 \mathrm{E}-02$ & & $6.0911 \mathrm{E}-02$ & \\
5 & 3 & $3.1050 \mathrm{E}-02$ & 1.9617 & $3.1050 \mathrm{E}-02$ & 1.9617 \\
6 & 3 & $1.5600 \mathrm{E}-02$ & 1.9904 & $1.5600 \mathrm{E}-02$ & 1.9904 \\
7 & 3 & $7.8094 \mathrm{E}-03$ & 1.9976 & $7.8094 \mathrm{E}-03$ & 1.9976 \\
\hline
\end{tabular}

Tabela 5.2: Erro e ordem de convergência no cálculo dos valores das células fantasmas empregando o produto matriz-vetor e o cálculo usual nível-a-nível, retalho-a-retalho. Condição de contorno de Neumann.

\begin{tabular}{cccccc}
\hline $\mathrm{n}$ & $\mathrm{L}$ & $\left|\varphi_{e}-\varphi_{\text {MatVec }}\right|_{\infty}$ & $r_{\infty}$ & $\left|\varphi_{e}-\varphi_{\text {SAMR }}\right|_{\infty}$ & $r_{\infty}$ \\
\hline 4 & 3 & $3.7317 \mathrm{E}-04$ & - & $3.7317 \mathrm{E}-04$ & - \\
5 & 3 & $4.6802 \mathrm{E}-05$ & 7.9734 & $4.6802 \mathrm{E}-05$ & 7.9734 \\
6 & 3 & $5.8550 \mathrm{E}-06$ & 7.9935 & $5.8550 \mathrm{E}-06$ & 7.9935 \\
7 & 3 & $7.3203 \mathrm{E}-07$ & 7.9984 & $7.3203 \mathrm{E}-07$ & 7.9984 \\
\hline
\end{tabular}

Tabela 5.3: Erro e ordem de convergência no cálculo dos valores das células fantasmas empregando o produto matriz-vetor e o cálculo usual nível-a-nível, retalho-a-retalho. Condição de contorno periódica. 
poladores que usamos para aproximar as células fantasmas com condição de contorno de Dirichlet, de Neumann e periódica, respectivamente.

Calegari [25] apresentou resultados onde o cálculo para aproximar células fantasmas é a operação mais chamada para cada passo de tempo na solução de escoamentos reativos com baixo número de Mach. O custo computacional total apresentado nesse cálculo é alto, especialmente quando temos um número elevado de retalhos por cada nível de refinamento. Uma vantagem que obtemos com a estrutura matricial é que ela armazena o estêncil para o cálculo de valores nas células fantasmas e isto é feito uma única vez por remalhagem.

Também uma única representação matricial para o cálculo de valores nas células fantasmas serve para aproximar tais valores de qualquer variável de mesmo tipo, e.g. no centro de célula (massa específica, viscosidade e correção de pressão, entre outras). De maneira semelhante, podemos empregar uma única representação matricial para valores fantasmas de variáveis definidas no meio de arestas.

\subsubsection{Tempo computacional do cálculo matricial de valores fantasmas}

Interessados no tempo de cômputo para obtermos valores em células fantasmas com a estrutura matricial, para uma variável definida no centro de célula com valores visíveis dados pela função (5.6), mostramos nas tabelas 5.4 e 5.6 tais tempos para a montagem da estrutura matricial que tem o estêncil para o cálculo de células fantasmas, o tempo gasto para obter os valores via produto matrizvetor e o tempo gasto com o cálculo usual nível-a-nível, retalho-a-retalho.

Este teste foi separado em dois casos. Um para condição de contorno de Neumann e outro para condição periódica. Cada um deles foi realizado para diferentes malhas, as mostradas nas figuras 5.2 e 5.3, as quais foram obtidas a partir de uma malha inicial aumentando-se consecutivamente um a um os níveis de refinamento.

O primeiro caso para o qual comparamos os tempos computacionais dados na obtenção de células fantasmas com as duas metodologias é para condição de contorno de Neumann. Aqui usamos as malhas mostradas nas figuras 5.2 (a), (b) e (c). Mostramos na Tabela 5.4 os tempos computacionais de cada operação no caso de condição de contorno de Neumann. A primeira linha corresponde à malha com três níveis de refinamento e malha base da ordem $2^{7} \times 2^{7}$, G7L3, mostrada na Figura 5.2(a). Consecutivamente, a segunda linha é para a malha, G7L4, mostrada na Figura 5.2(b) e a última linha é para a malha G7L5 na Figura 5.2(c). Para cada uma destas malhas compostas, mostramos os retalhos correspondentes nos diferentes níveis nas figuras 5.2 (d), (e) e (f), respectivamente. Mostramos a quantidade de retalhos para cada um dos níveis destas malhas na Tabela 5.4.

\begin{tabular}{cccc}
\hline Malha & $\begin{array}{c}\text { t_montar_Mat } \\
(\mathrm{s})\end{array}$ & $\begin{array}{c}\mathrm{t} \text {-MatVec } \\
(\mathrm{s})\end{array}$ & $\begin{array}{c}\mathrm{t}_{\text {_calcghost }} \\
(\mathrm{s})\end{array}$ \\
\hline G7L3 & $2.8002 \mathrm{E}-02$ & 0.0000 & 0.0000 \\
G7L4 & $1.2401 \mathrm{E}-01$ & $4.0000 \mathrm{E}-03$ & $4.0000 \mathrm{E}-03$ \\
G7L5 & $2.9202 \mathrm{E}-01$ & $8.0010 \mathrm{E}-03$ & $1.2000 \mathrm{E}-02$ \\
\hline
\end{tabular}

Tabela 5.4: Tempos gastos no cálculo dos valores em células fantasmas: matriz-vetor e cálculo convencional, para condição de contorno de Neumann. As malhas compostas usadas, seguindo a ordem da tabela, estão na Figura 5.2.

Da mesma forma que no caso de condição de contorno de Neumann, mas agora para o caso 
(a)

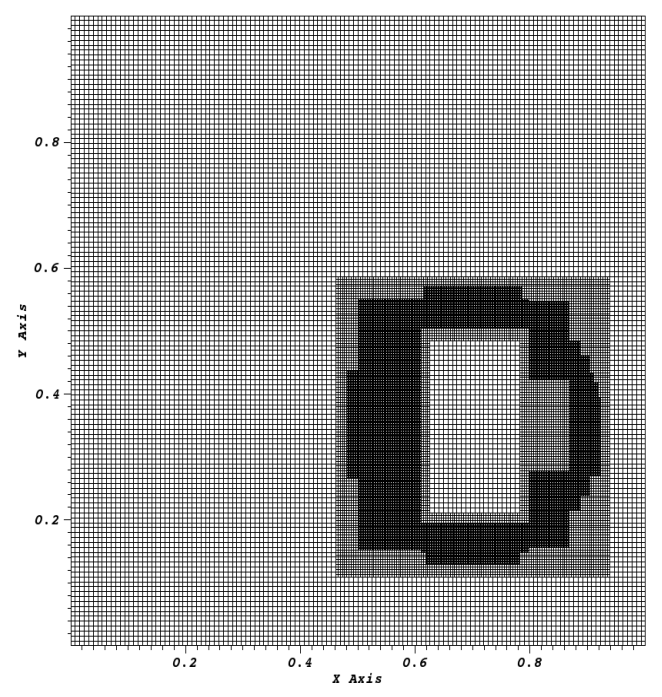

(b)

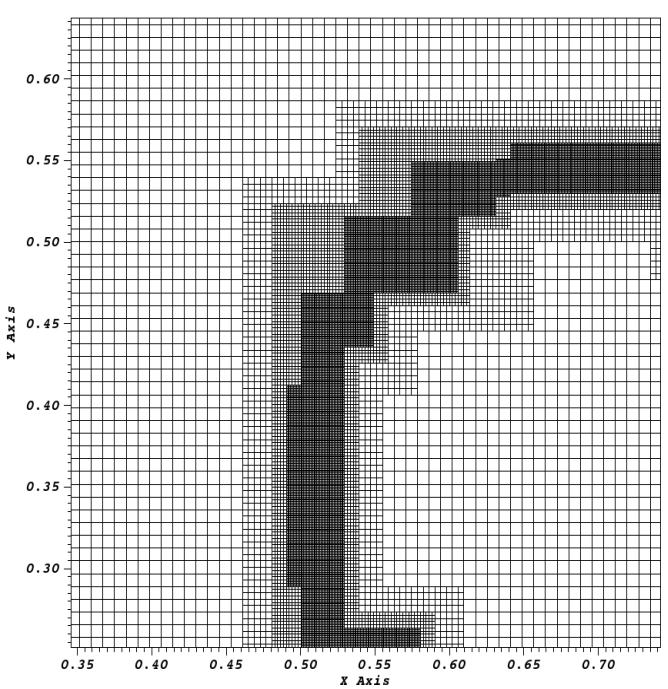

(c)

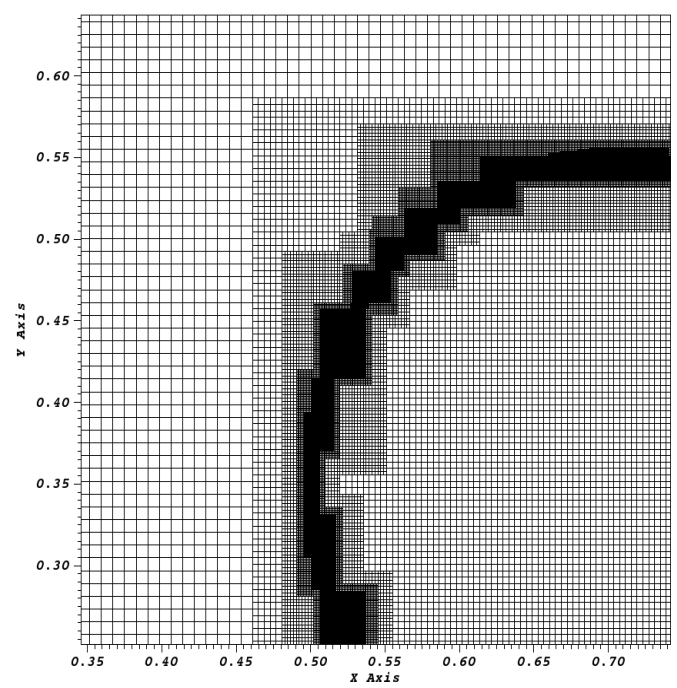

(d)

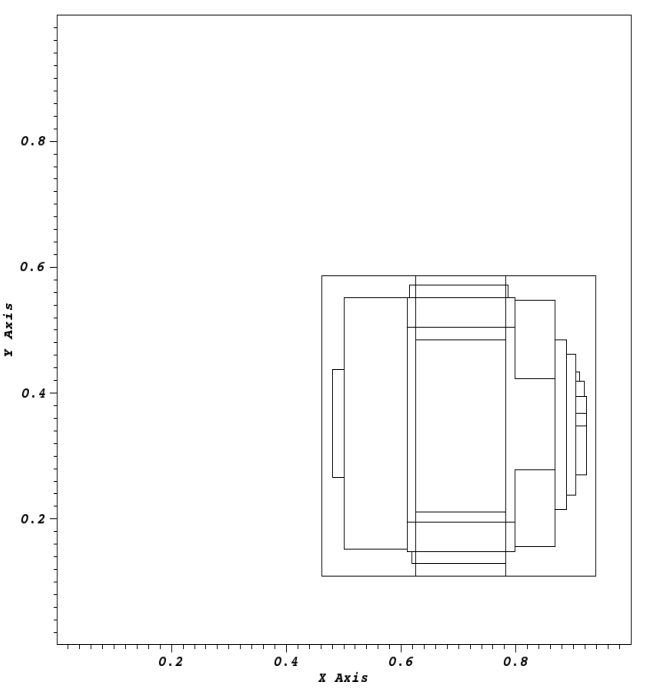

(e)
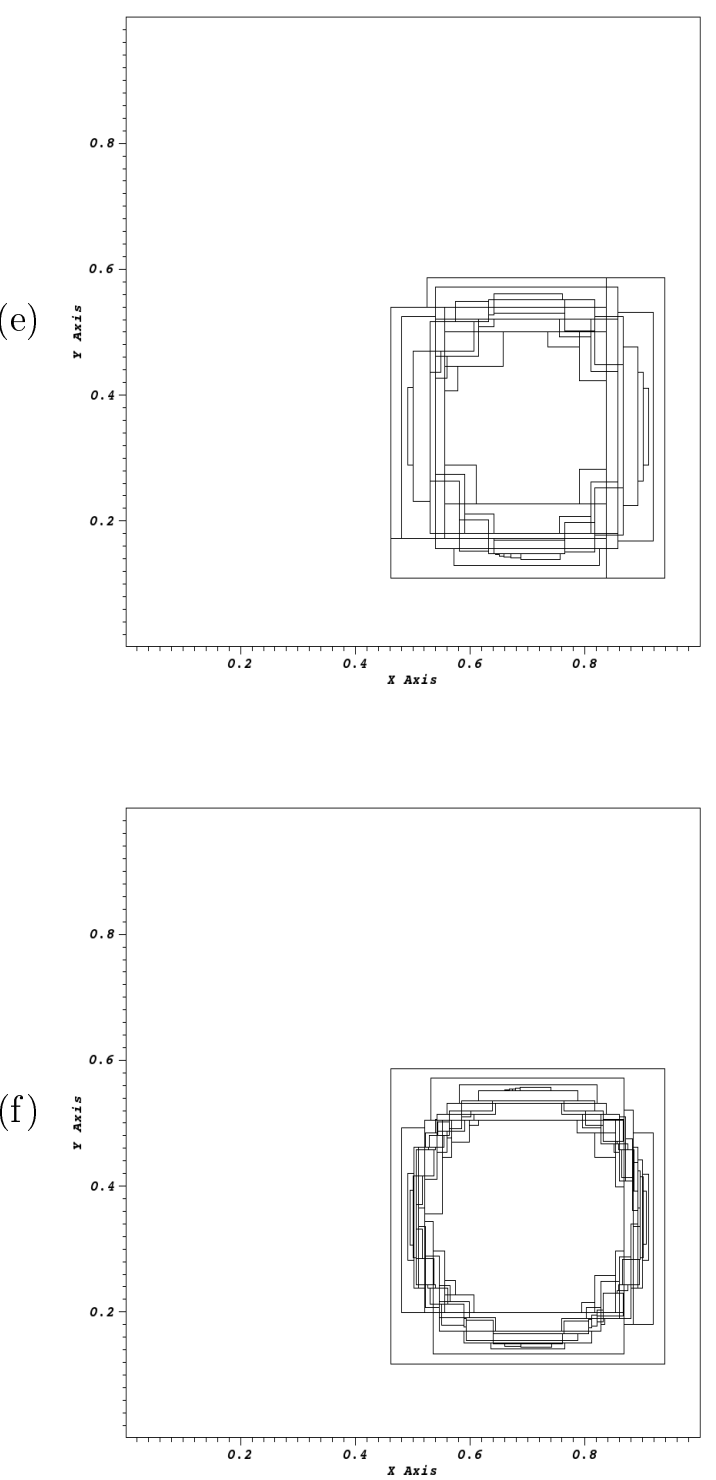

Figura 5.2: Malhas e retalhos usados no cálculo de valores em células fantasmas com condição de contorno de Neumann. 
(a) $\stackrel{n}{\frac{0}{x}}$
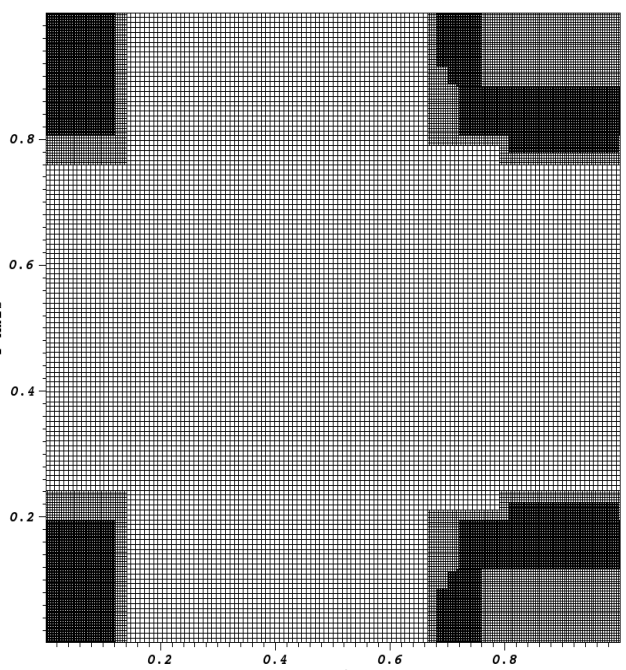

(b)

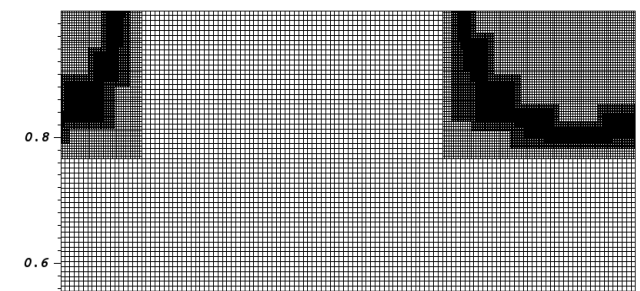

)

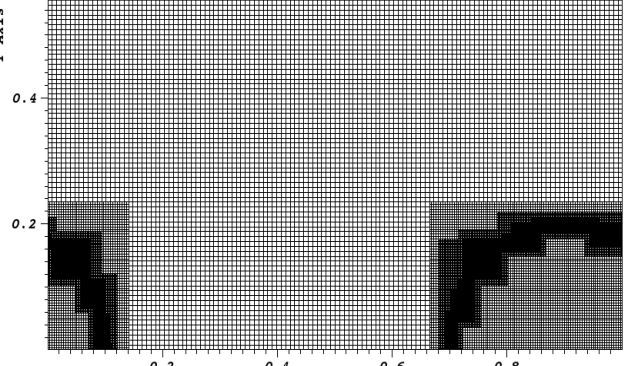

0.2

$0.4_{\mathrm{A} \text { axis }} 0.6$

(c)

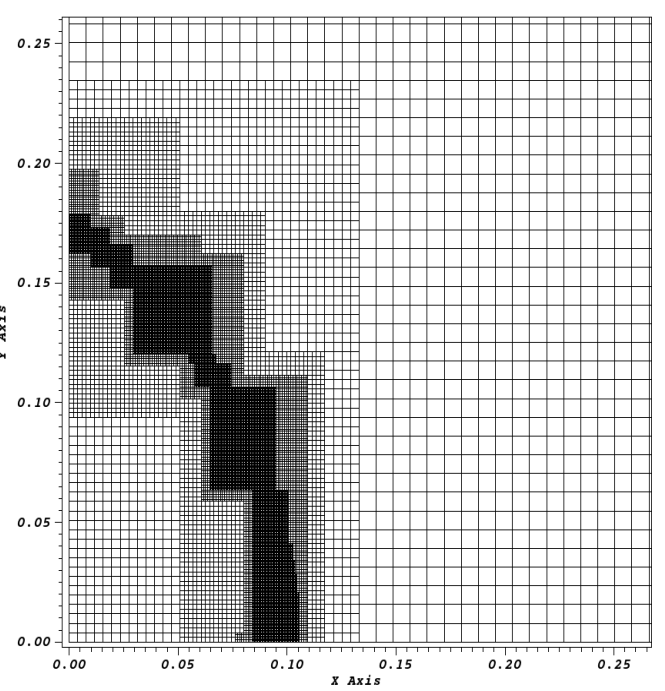

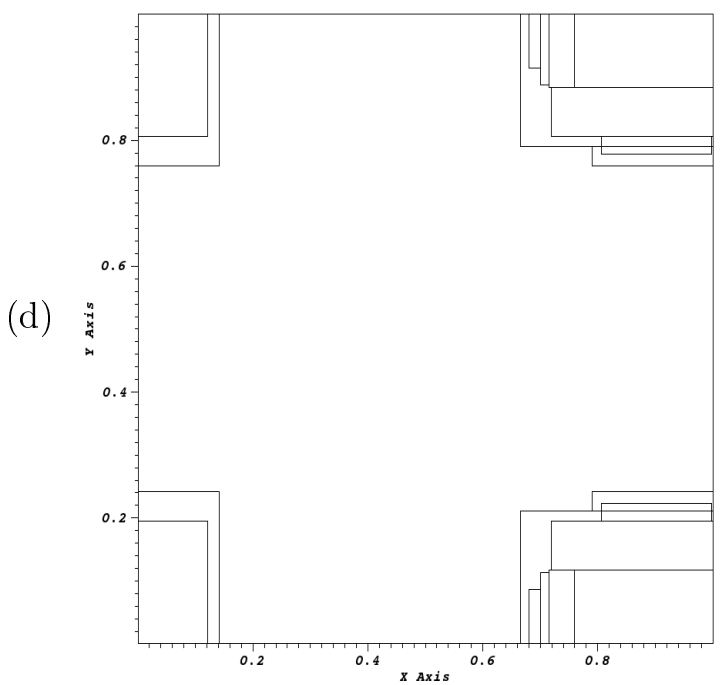
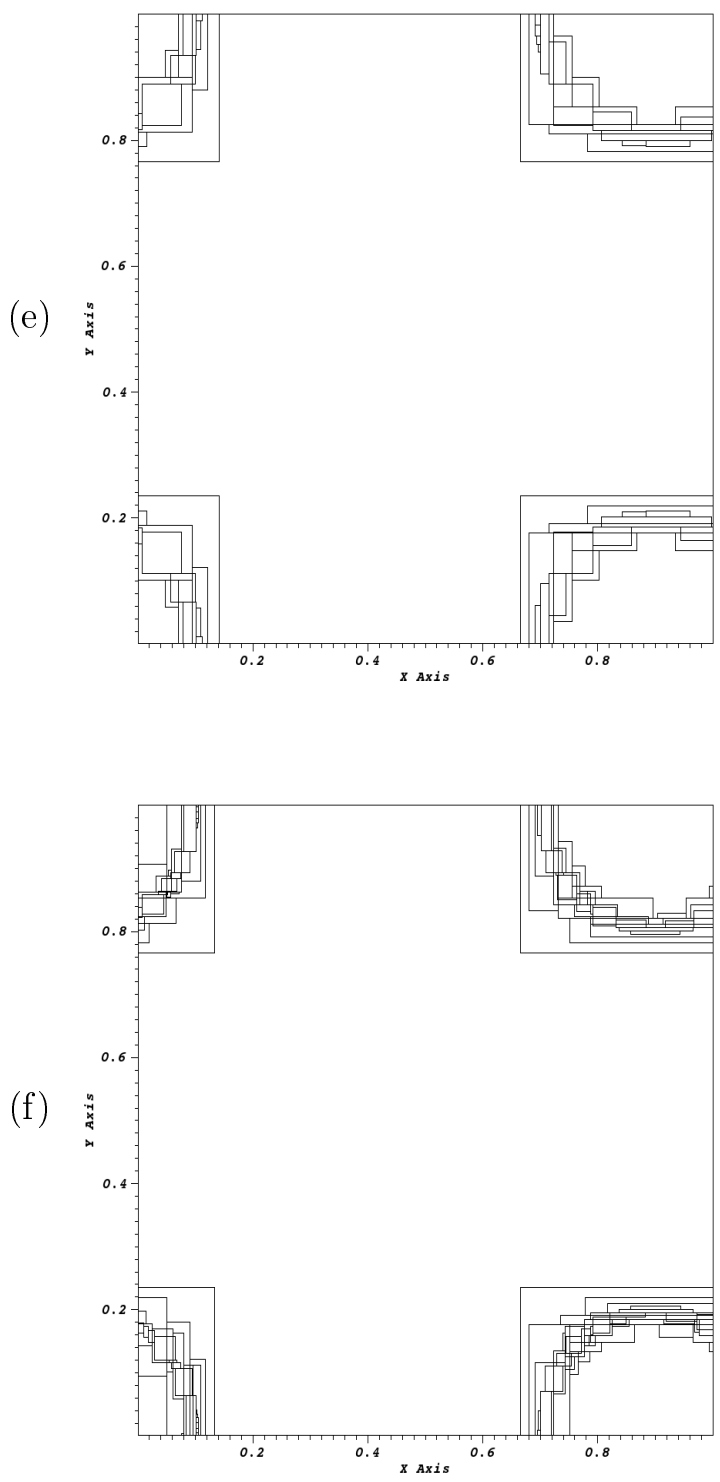

Figura 5.3: Malhas e retalhos usados no cálculo de células fantasmas, com condição de contorno periódica. 


\begin{tabular}{ccccccc}
\hline & $L_{1}$ & $L_{2}$ & $L_{3}$ & $L_{4}$ & $L_{5}$ & Total \\
\hline G7L3 & 1 & 4 & 15 & - & - & 20 \\
G7L4 & 1 & 12 & 14 & 24 & - & 51 \\
G7L5 & 1 & 1 & 19 & 34 & 48 & 103 \\
& & & & & & \\
\hline
\end{tabular}

Tabela 5.5: Informação sobre a quantidade de retalhos por nivel da Figura 5.2.

periódico, mostramos na Tabela 5.6 os tempos computacionais das diferentes formas de cálculo de valores em células fantasmas. As malhas compostas usadas neste caso para os valores obtidos para cada uma das três linhas da Tabela 5.6, são mostradas nas figuras 5.3(a)-(c), respectivamente. Os retalhos para todos os níveis de cada uma destas malhas são mostradas nas figuras 5.3(d)-(f), respectivamente, e a informação sobre a quantidade de retalhos em cada nível das malhas é dada na Tabela 5.7.

\begin{tabular}{cccc}
\hline Malha & t_montar_Mat $_{-}(\mathrm{s})$ & $\begin{array}{c}\mathrm{t} \_ \text {MatVec } \\
(\mathrm{s})\end{array}$ & $\begin{array}{c}\mathrm{t} \text { _calcghost } \\
(\mathrm{s})\end{array}$ \\
\hline G7L3 & $3.6002 \mathrm{E}-02$ & $4.0000 \mathrm{E}-03$ & 0.0000 \\
G7L4 & $1.0801 \mathrm{E}-01$ & $4.0000 \mathrm{E}-03$ & $4.0010 \mathrm{E}-03$ \\
G7L5 & $3.1602 \mathrm{E}-01$ & $8.0010 \mathrm{E}-03$ & $1.6001 \mathrm{E}-02$ \\
\hline
\end{tabular}

Tabela 5.6: Tempos gastos no cálculo dos valores em células fantasmas: matriz-vetor e cálculo convencional, para condição de contorno periódica em ambas as direções. As malhas compostas usadas, seguindo a ordem da tabela, estão na Figura 5.3.

\begin{tabular}{ccccccc}
\hline & $L_{1}$ & $L_{2}$ & $L_{3}$ & $L_{4}$ & $L_{5}$ & Total \\
\hline G7L3 & 1 & 6 & 12 & - & - & 19 \\
G7L4 & 1 & 4 & 24 & 33 & - & 62 \\
G7L5 & 1 & 4 & 26 & 41 & 55 & 127 \\
\hline
\end{tabular}

Tabela 5.7: Informação sobre a quantidade de retalhos por nível da Figura 5.3.

Ignorando o tempo da montagem da estrutura matricial para aproximação de valores em células fantasma, tanto para o caso com condição de Neumann na Tabela 5.4, como no caso com condição periódica na Tabela 5.6, percebemos que ao aumentarmos a quantidade de níveis e de retalhos, o tempo computacional, t_MatVec, no cálculo de células fantasmas com o produto matriz-vetor diminui quando comparado com o tempo computacional do calculo usual t_calcghost para determinar as células fantasmas. Isto traz grandes vantagens quando comparado com a necessidade de aproximar células fantasmas para diferentes variáveis definidas na mesma posição de célula, durante diferentes instantes de tempo nos quais se tenha a mesma malha. O tempo de se montar a estrutura matricial é gasto uma vez por remalhagem e por isso, como a cálculo de valores fantasmas é feito dezenas de vezes mais, ele foi excluído.

Devemos lembrar que o cálculo do produto matriz-vetor embora só envolva elementos diferentes de zero, ao usarmos na estrutura de dados atual listas ligadas para armazenarmos a matriz, este produto acaba se tornando mais caro computacionalmente se considerarmos um produto matriz-vetor usando uma estrutura vetorial. O processamento de uma lista ligada tem um tempo computacional 
maior do que o processamento vetorial [89]. Com a experiência adquirida na criação da matriz com essas estruturas, podemos passar a usar uma estrutura vetorial seguindo o formato original para armazenamento de matriz esparsa CSR[87, 88]. A estrutura vetorial dá vantagens na diminuição do tempo de processamento dos elementos armazenados nela e facilitará a comunicação para o caso paralelo. Prevemos a alteração da forma de armazenamento em um trabalho futuro próximo.

A estrutura com lista ligada foi pensada inicialmente porque não se tinha ideia da quantidade máxima de células de outros ou do mesmo nível que poderia estar envolvida em uma interpolação (do tamanho do estêncil de interpolação e de discretização). Ao iniciar o trabalho com matrizes, era importante preenchermos os valores dos níveis recobertos com médias, para permitir as operações usuais retalho-a-retalho entre células visíveis e invisíveis. Esta operação carregava informação que envolvia muitas células de diferentes níveis e o uso de listas ligadas permitia a inclusão de novos elementos de maneira versátil. Percebemos com o tempo que são importantes para as operações apenas as células visíveis e, com isto, ganhamos mais controle sobre a quantidade de células envolvidas nas interpolações, viabilizando o uso de uma estrutura vetorial.

Por outro lado, percebemos que uma grande vantagem do cálculo de valores em células fantasmas com o produto matriz-vetor é o fato dessa montagem ser realizada uma única vez para cada malha composta gerada e é a mesma para quaisquer variáveis localizadas nas mesmas posições, diferenciando-se cada variável apenas pela condição de contorno que lhe corresponde. Decorre destas observações que ao termos paralelismo com uma malha composta distribuída, a comunicação entre processos para o cálculo destas células vai diminuir consideravelmente. Ela dependerá basicamente da comunicação necessária para realizar o produto matriz-vetor e de condições de contorno [90].

Uma outra vantagem de termos uma estrutura matricial para o cálculo de células fantasmas é que o paralelismo pode ser explorado mesmo em casos em que o resto do código é sequencial (já ganhamos acesso ao paralelismo via uso de bibliotecas com paralelização nativa). Em casos nos quais já teremos também a malha composta distribuída em diferentes processos [90], o cálculo dos valores em células fantasmas tipicamente impõe muita comunicação entre os diferentes processos, diminuindo a escalabilidade da técnica SAMR. A eficiência do produto matriz-vetor em paralelo tem sido muito estudada e há varias técnicas para realizar este cálculo [20, 61, 87]. Portanto, acreditamos que o cálculo das células fantasmas com o produto matriz-vetor será de grande vantagem também para o SAMR que tem a malha composta construída também em paralelo.

Como o uso de uma matriz para representar uma discretização de um operador diferencial em malha composta foi concebido completamente por nós e é um dos resultados principais obtidos no presente trabalho, devemos fazer uma verificação detalhada e cuidadosa de tal estrutura. Primeiro, estudamos o caso da discretização para variáveis definidas em centro de célula, seguido do caso da discretização para variáveis definidas no meio das arestas. O uso da estrutura matricial na discretização de problemas-modelo de equações a derivadas parciais lineares, fundamentais aos nossos planos, é apresentado nas seções subsequentes.

\subsection{Resolução matricial de equações diferenciais parciais}

Para obter a solução da equação de Stokes na malha composta, iniciamos nosso estudo por meio de uma técnica de discretização que acoplava as variáveis em um único sistema linear que iria ser solucionado via multinível-multigrid [2, 72]. Este sistema incluia um bloco de zeros na 
diagonal oriundo da condição de incompressibilidade e que impediu o uso como relaxador para o multigrid do Método de Gauss-Seidel Preto-Vermelho [104]. Tal impedimento levou à necessidade de buscarmos uma outra abordagem, de termos acesso a outros métodos. Várias tentativas foram realizadas, inicialmente em malha uniforme, e dentre as implementações de um Gauss-Saidel multicolorido [104], do Método SIMPLE [76] e o do Método de Uzawa para a equação de Stokes [4, 7, $56,57]$, obtivemos sucesso com o último.

Em malha composta, a implementação do Método de Uzawa para a equação de Stokes, que emprega o Método BiCG-Stab, apresentou problemas na convergência ao calcularmos os valores fantasmas da maneira convencional em malha composta, empregando a implementação original por nível-a-nível, retalho-a-retalho. A tentativa de descobrir as razões para o comportamento divergente foi um processo bastante educativo e levou a um conhecimento profundo do algoritmo de cálculo de fantasmas em sua versão original. Tal comportamento era ainda mais intrigante quando se encontrava na literatura a implementação do Método de Uzawa em malhas adaptativas não estruturadas voltadas a elementos finitos $[40,56,59,69]$ e nenhum relato de problemas com a convergência do método. Suspeitávamos que o mau comportamento com relação à convergência era causado pela maneira de como as interpolações estavam sendo feitas em células fantasmas no SAMR. A busca pela forma matricial permitiu entender claramente como estas eram formadas entre as malhas dos diferentes níveis. O cálculo de valores fantasmas da maneira tradicional, nível-a-nível, retalho-a-retalho, depende do uso de posições auxiliares de memória que são acionadas diversas vezes para fins diferentes durante o processo de interpolação. Isto torna difícil saber exatamente quais são os pesos finais empregados na obtenção de cada valor fantasma. Tal entendimento permitiu que alterássemos a implementação original e que, por fim, conseguíssemos o funcionamento do Método BiCG-Stab em malhas compostas, implementado com uma estratégia nível-a-nível, retalho-a-retalho.

Para verificar a implementação matricial empregando o PETSc para a solução de sistemas lineares, abordamos primeiro a solução da equação de Poisson para variáveis centradas na células. Na solução da equação de Stokes pelo Método de Uzawa com BiCG-Stab é necessária a solução de um sistema linear oriundo de uma equação elíptica nas arestas, como mostramos na Seção 3.5. Entretanto, como primeiro teste de nossos desenvolvimentos, achamos natural iniciarmos o estudo para o caso de variáveis definidas no centro de célula por ser a implementação muito mais simples. Ao mesmo tempo, corroborou o fato de estarmos também interessados no uso de bibliotecas externas na solução de Navier-Stokes pois, como mencionamos anteriormente, não é incomum problemas com a convergência do método ao empregarmos métodos multinível-multigrid em problemas bifásicos com altas razões de massa específica [111]. A necessidade de maior robustez nestes escoamentos transientes bifásicos também catalizou a decisão de abordarmos centros de células em primeiro lugar, sendo posteriormente o foco dirigido às equações elípticas nos meios de arestas. Apresentamos os resultados obtidos para equações elípticas em centros de células e em meios de aresta nas seções 5.3.1 e 5.3.2, respectivamente.

O uso de uma representação matricial explícita abre também a possibilidade de se pensar na solução da equação de Navier-Stokes com o Método de Projeção segregado ou monolítico [82], acoplando e desacoplando a correção de pressão das velocidades. Isso justifica o estudo de outro problema-modelo: um problema parabólico nas arestas (Seção 5.3.3). Desta maneira, teremos assim a opção de futuramente tentar uma solução matricial para a equação de Navier-Stokes no caso de escoamentos transientes. 


\subsubsection{Primeiro problema-modelo: equação elíptica para variáveis centradas}

Consideremos a equação de Poisson com coeficientes variáveis e condições de contorno quaisquer,

$$
\nabla \cdot(\omega(x, y) \nabla \varphi(x, y))=f(x, y), \quad(x, y) \in \Omega
$$

condições de contorno em $\partial \Omega$ : de Dirichlet, periódicas ou de Neumann,

onde $f(x, y)$ é o termo forçante, $\Omega$ é o domínio espacial, aqui adotado como sendo $[0,1] \times[0,1]$, $\partial \Omega$ é a fronteira do domínio espacial, $\omega(x, y)$ é uma função que será definida nos testes e $\varphi$ é a incógnita.

Sejam $\varphi$ e $\omega$ definidas no centro da célula computacional. Quando as condições de contorno são puramente periódicas ou de Neumann, ou uma mistura destas duas, existe uma condição de compatibilidade [65] bem conhecida e que deve ser tratada com cuidado para que tenhamos a solução do problema discretizado. Satisfazer esta condição de compatibilidade equivale a termos uma versão discreta do Teorema de Gauss satisfeita na malha e está também associada à falta de unicidade de solução. Quando temos ao menos uma condição de contorno do tipo Dirichlet não há problema de unicidade de solução e, em geral, implementamos com muito mais facilidade a resolução numérica do sistema discretizado.

No espírito do exposto acima, abordaremos o problema (5.7) de duas formas diferentes: uma que pressupõe a existência de pelo menos uma condição de contorno do tipo Dirichlet e outra na qual passaremos, de fato, ao estudo da solução via forma matricial com uma discretização que aceite condições de contorno gerais (e que satisfaz, portanto, a forma discreta do Teorema de Gauss). A vantagem de apresentarmos desta maneira é tornar a exposição de ideias mais simples, didática e respeitar a ordem cronológica real de como as técnicas foram sendo desenvolvidas.

A quantidade de células fantasmas que usamos em todos os testes desta seção é "um" (entendase: "uma camada, uma moldura de uma célula computacional adicional no entorno de cada retalho de malha composta").

\section{Equação elíptica para variáveis centradas: pelo menos uma condição de Dirichlet}

Para obter a discretização da equação (5.7) com pelo menos uma condição de contorno de Dirichlet, expandimos cada um dos termos do divergente obtendo

$$
\nabla \cdot(\omega \nabla \varphi)=\omega \nabla^{2} \varphi+\partial_{x} \omega \partial_{x} \varphi+\partial_{y} \omega \partial_{y} \varphi
$$

da qual se vê facilmente a redução do problema quando temos $\omega$ constante.

Com as variáveis $\varphi$ e $\omega$ definidas no centro de célula, a discretização de (5.8), supondo pelo menos uma condição de contorno de Dirichlet, é dada por

$$
\begin{aligned}
\omega_{i j}\left(\nabla^{2} \varphi\right)_{i j} & +\frac{\omega_{i+1 j}-\omega_{i-1 j}}{2 \Delta x} \frac{\varphi_{i+1 j}-\varphi_{i-1 j}}{2 \Delta x}+ \\
& +\frac{\omega_{i j+1}-\omega_{i j-1}}{2 \Delta y} \frac{\varphi_{i j+1}-\varphi_{i j-1}}{2 \Delta y}=f_{i, j}
\end{aligned}
$$

onde

$$
\left(\nabla^{2} \varphi\right)_{i j}=\frac{\varphi_{i+1, j}-2 \varphi_{i j}+\varphi_{i-1, j}}{\Delta x^{2}}+\frac{\varphi_{i, j+1}-2 \varphi_{i j}+\varphi_{i, j-1}}{\Delta y^{2}}
$$


é a discretização usual para o operador laplaciano.

De (5.9), obtemos os valores que correspondem a cada posição da matriz e assim a forma matricial que obtemos para cada linha é

$$
\begin{gathered}
\left(\frac{\omega_{i j}}{\Delta x^{2}}-\frac{\omega_{i+1 j}-\omega_{i-1 j}}{4 \Delta x^{2}}\right) \varphi_{i-1 j}+\left(\frac{\omega_{i j}}{\Delta y^{2}}-\frac{\omega_{i j+1}-\omega_{i j-1}}{4 \Delta y^{2}}\right) \varphi_{i j-1}+ \\
+\left[-2 \omega_{i j}\left(\frac{1}{\Delta x^{2}}+\frac{1}{\Delta y^{2}}\right)\right] \varphi_{i j}+\left(\frac{\omega_{i j}}{\Delta y^{2}}+\frac{\omega_{i j+1}-\omega_{i j-1}}{4 \Delta y^{2}}\right) \varphi_{i j+1}+ \\
+\left(\frac{\omega_{i j}}{\Delta x^{2}}+\frac{\omega_{i+1 j}-\omega_{i-1 j}}{4 \Delta x^{2}}\right) \varphi_{i+1 j}=f_{i j} \\
A \varphi=\mathbf{f} .
\end{gathered}
$$

Na Figura 5.4 temos o gráfico do padrão de esparsidade da matriz para esta discretização (5.11) em uma malha uniforme de $n_{x} \times n_{y}$ células e para a malha composta da Figura 5.5 com dois níveis de refinamento. O padrão na Figura 5.4 representa as posições com valores não nulos da matriz. Observamos que o fato de $\omega$ ser ou não constante, não muda o padrão de esparsidade da matriz que representa a discretização (5.11).
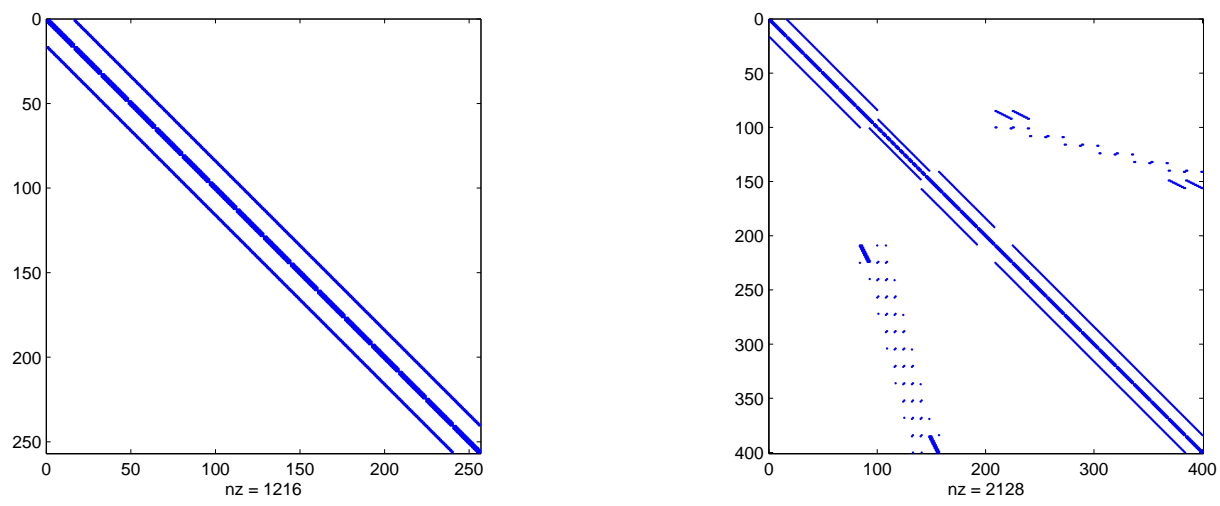

Figura 5.4: Padrão de esparsidade da discretização (5.11) na malha uniforme e na malha composta $L_{2}$ da Figura 5.5.

A biblioteca PETSc inclui vários formatos para o armazenamento da matriz esparsas. Inicialmente, em nossos testes, não foi fixado nenhum em particular e a biblioteca usou seu default. Isto gerou matrizes densas que tomavam um tempo grande para serem processadas. Optamos então pelo uso do formato esparso AIJ [88], que é definido para o caso sequencial por MATSEQAIJ e, para o caso paralelo, por MATMPIAIJ.

Alguns testes realizados são descritos a seguir, nos quais a solução do sistema linear dado pela discretização da equação de Poisson (5.11) for computada com o uso do PETSc em um único processo, com o método GMRES e pré-condicionador ILU(0) (default no PETSc). Adotamos tolerância de $10^{-12}$, número máximo de iterações de $10^{4}$ e não fixamos o tipo de representação da matriz usada para armazenar a discretização na estrutura do PETSc, ficando assim a representação default do aplicativo. 
As funções usadas para os testes são:

$$
\begin{aligned}
\varphi(x, y) & =\sin (2 \pi x) \sin (2 \pi y) \\
f(x, y) & =\omega \nabla^{2} \varphi+\partial_{x} \omega \partial_{x} \varphi+\partial_{y} \omega \partial_{y} \varphi \quad \text { e } \\
\omega(x, y) & =(1+h)+h * \tanh (2 \pi d(x-0.5)) \text { em }[0,1] \times[0,1]
\end{aligned}
$$

onde $h$ controla a variação de $\omega$ e $d$ a quantidade de células na qual esta variação sucede.

No primeiro teste empregamos para $h=0$ (sem "salto de propriedade"). Assim sendo, $\omega(x, y)=1$ e $f(x, y)=-8 \pi^{2} \sin (2 \pi x) * \sin (2 \pi y)$. A malha composta empregada tem dois níveis de refinamento e está mostrada na Figura 5.5, junto à aproximação obtida para $\varphi(x, y)$.

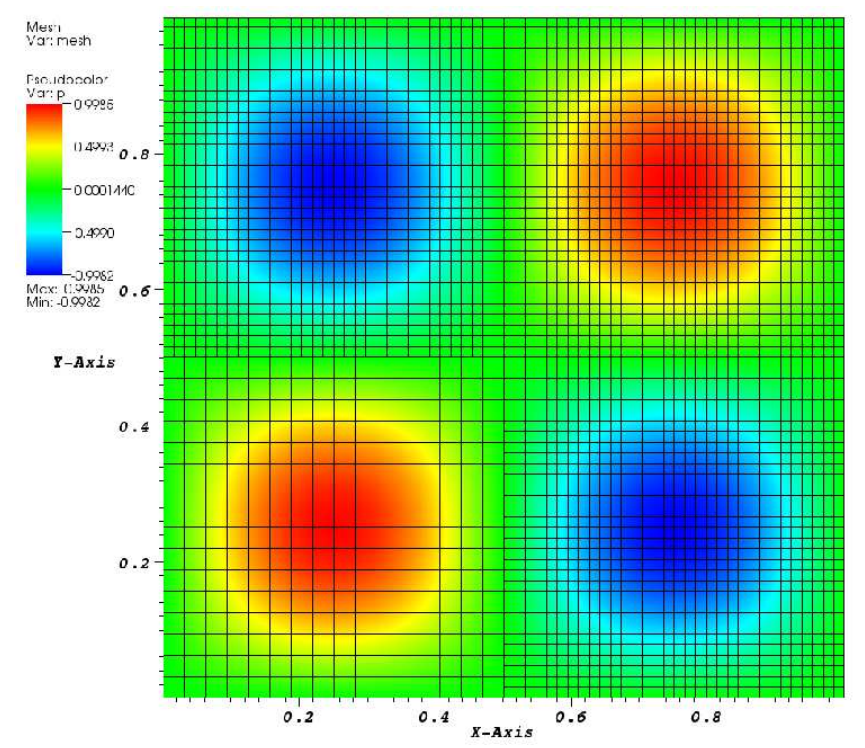

Figura 5.5: Malha composta com dois niveis de refinamento e solução obtida com o PETSc.

A ordem esperada para a discretização é dois, a qual foi comprovada numericamente. Os resultados obtidos estão na Tabela 5.8 para o caso uniforme e, na Tabela 5.9, para a malha composta da Figura 5.5. A coluna "Iter" nas tabelas 5.8 e 5.9 fornece a quantidade de iterações que o Método GMRES, com précondicionador ILU(0) do PETSc, precisou para determinar a solução do sistema com precisão $10^{-12}$.

\begin{tabular}{cccccc}
\hline Malha & $\|e\|_{\infty}$ & $r_{\infty}$ & $\|e\|_{2}$ & $r_{2}$ & Iter \\
\hline $32 \times 32$ & $3.1880 \mathrm{E}-003$ & & $1.60948 \mathrm{E}-003$ & & 36 \\
$64 \times 64$ & $8.0164 \mathrm{E}-004$ & 3.9769 & $4.01789 \mathrm{E}-004$ & 4.0058 & 88 \\
$128 \times 128$ & $2.0070 \mathrm{E}-004$ & 3.9942 & $1.00411 \mathrm{E}-004$ & 4.0014 & 272 \\
$256 \times 256$ & $5.0193 \mathrm{E}-005$ & 3.9985 & $2.51004 \mathrm{E}-005$ & 4.0004 & 724 \\
$512 \times 512$ & $1.2549 \mathrm{E}-005$ & 3.9996 & $6.27497 \mathrm{E}-006$ & 4.0000 & 2653 \\
\hline
\end{tabular}

Tabela 5.8: Erro, ordem de convergência e número de iterações para a equação de Poisson em malha uniforme. Variáveis no centro de célula.

Caracterizamos as matrizes esparsas obtidas. Os valores calculados para o condicionamento da matriz nas malhas uniforme e composta estão na Tabela 5.10. O gráfico de esparsidade, os autovalores da matriz $A$, os autovalores da matriz $A^{T} A$ (estudados pelo interesse em usar o Método BiCG-Stab) e o número de condicionamento da matriz, foram calculados usando funções do MAT- 


\begin{tabular}{cccccc}
\hline Malha & $\|e\|_{\infty}$ & $r_{\infty}$ & $\|e\|_{2}$ & $r_{2}$ & Iter \\
\hline $32 \times 32 L 2$ & $3.54768 \mathrm{E}-003$ & & $1.00282 \mathrm{E}-003$ & & 133 \\
$64 \times 64 L 2$ & $8.82303 \mathrm{E}-004$ & 4.0209 & $2.48572 \mathrm{E}-004$ & 4.0343 & 299 \\
$128 \times 128 L 2$ & $2.19873 \mathrm{E}-004$ & 4.0128 & $6.19008 \mathrm{E}-005$ & 4.0156 & 952 \\
$256 \times 256 L 2$ & $5.49076 \mathrm{E}-005$ & 4.0044 & $1.54464 \mathrm{E}-005$ & 4.0074 & 3340 \\
$512 \times 512 L 2$ & $1.37184 \mathrm{E}-005$ & 4.0025 & $3.85811 \mathrm{E}-006$ & 4.0036 & 9580 \\
\hline
\end{tabular}

Tabela 5.9: Erro, ordem de convergência e número de iterações para a equação de Poisson em malha composta com dois niveis. Variáveis no centro de células.

LAB. Empregamos para isto uma rotina que imprime a matriz armazenada no AMR2D no formato de matriz esparsa utilizada pelo GNU Octave ${ }^{3}$.

\begin{tabular}{cccccc}
\hline Malha & Dimensão & & Autovalor $(A)$ & Autovalor $\left(A^{T} A\right)$ & $K_{2}(A)$ \\
\hline $32 \times 32$ & \multirow{2}{*}{$1024 \times 1024$} & Mínimo & $-8.19198 \mathrm{e}+03$ & $6.64657 \mathrm{e}+07$ & $9.90416 \mathrm{e}-01$ \\
& & Máximo & $-8.15264 \mathrm{e}+03$ & $6.71089 \mathrm{e}+07$ & \\
\hline $64 \times 64$ & \multirow{2}{*}{$4096 \times 4096$} & Mínimo & $-3.27680 \mathrm{e}+04$ & $1.07116 \mathrm{e}+09$ & $9.97594 \mathrm{e}-01$ \\
& & Máximo & $-3.27285 \mathrm{e}+04$ & $1.07374 \mathrm{e}+09$ & \\
\hline \hline $32 \times 32 L 2$ & \multirow{2}{*}{$3328 \times 3328$} & mínimo & $-3.27621 \mathrm{e}+04$ & $1.06813 \mathrm{e}+09$ & $9.95137 \mathrm{e}-01$ \\
& & máximo & $-3.26823 \mathrm{e}+04$ & $1.07335 \mathrm{e}+09$ & \\
\hline $64 \times 64 L 2$ & \multirow{2}{*}{$13312 \times 13312$} & mínimo & $-1.31066 \mathrm{e}+05$ & $1.71574 \mathrm{e}+10$ & $9.98782 \mathrm{e}-01$ \\
& & máximo & $-1.30986 \mathrm{e}+05$ & $1.71783 \mathrm{e}+10$ & \\
\hline
\end{tabular}

Tabela 5.10: Informação da matriz para malha uniforme e composta com 2 níveis de refinamento da Figura 5.5.

A malha composta para os testes tem dois níveis de refinamento: o nível base é formado por um único retalho e o nível fino, acima, pela união de dois retalhos, como mostra a Figura 5.5. Também temos o gráfico com o comportamento do erro da solução obtida com o PETSc para esta malha na Figura 5.6 e, como esperado, ele é maior na região onde estão definidas as células mais grossas e menor nas finas.

Repetimos a resolução da equação de Poisson para variáveis no centro empregando uma malha composta mais complexa, contendo vários níveis de refinamento e união de retalhos, mostrada na Figura 5.7. A ideia é verificar que a solução não se degenera pelas diferentes interpolações usadas para a comunicação dos retalhos do mesmo ou de diferentes níveis de refinamento.

A malha usada para este teste é composta por sete níveis de refinamento e malha base $32 \times 32$, G5L7. A principal informação sobre as células da malha está na Tabela 5.11. Temos respectivamente, do nível de base ao nível mais fino de refinamento, as seguintes quantidades de retalhos: 1, 1, 1, 1, 19,34 e 48.

\begin{tabular}{ccccc}
\hline malha & total & visíveis & fantasmas & recobertas \\
\hline G5L7 & 229484 & 155380 & 22652 & 51452 \\
\hline
\end{tabular}

Tabela 5.11: Informação sobre a quantidade de células da malha composta com sete níveis de refinamento.

O valor obtido para o erro neste caso está na Tabela 5.12. A solução também foi dada pelo

\footnotetext{
${ }^{3}$ http://www.gnu.org/software/octave/
} 
Erro Sol. Poisson mateicial em malha composta

Malha:32X32L2

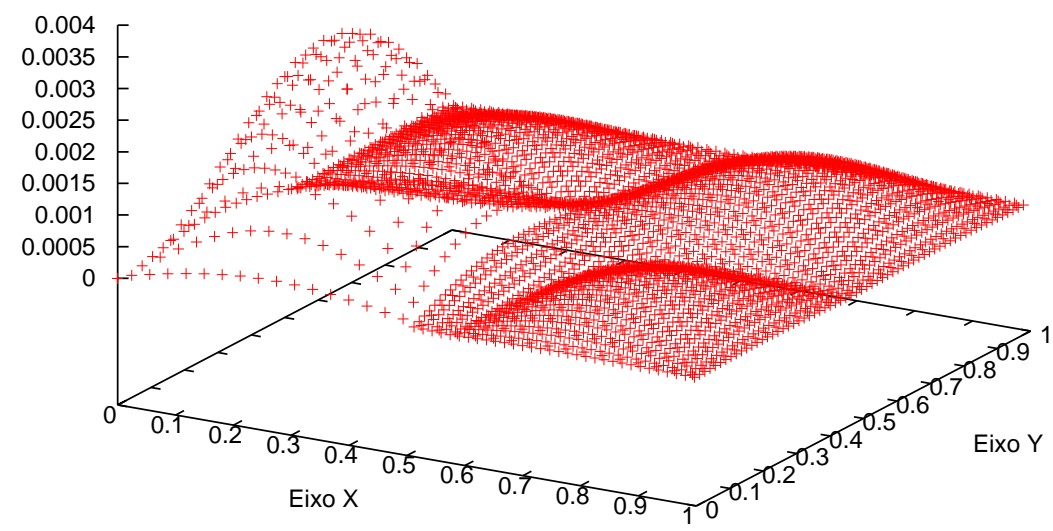

Figura 5.6: Erro na resolução da equação de Poisson com dois niveis de refinamento.

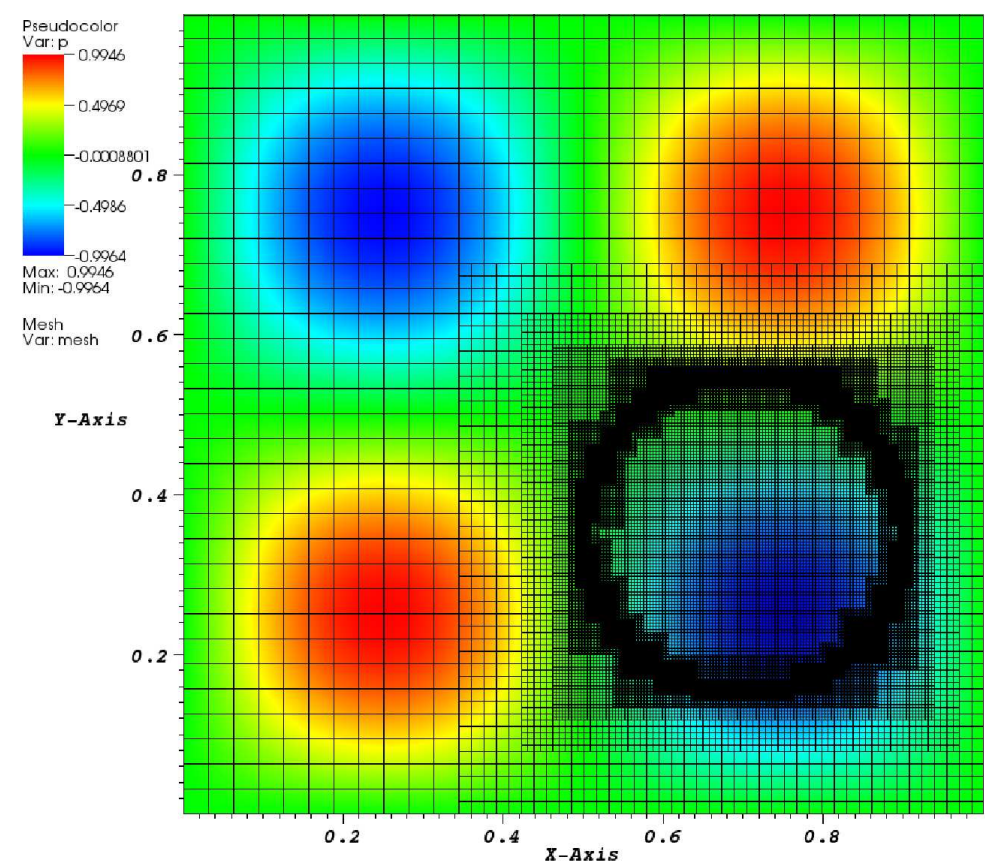

Figura 5.7: Malha composta com sete niveis de refinamento. 
GMRES com précondicionar ILU(0) e tolerância de $10^{-12}$ do PETSc. Foram necessárias 4.488 iterações nesse caso onde a matriz tem dimensão $155.380 \times 155.380$ (dada pelo número de células visíveis). Quando comparado com o erro obtido com o caso uniforme na Tabela 5.8, vemos que o uso da malha composta não degenerou a qualidade da aproximação obtida (esse era o resultado esperado uma vez que o problema tem solução muito suave).

\begin{tabular}{cccc}
\hline Malha & $\|e\|_{\infty}$ & $\|e\|_{2}$ & Iter \\
$32 \times 32 L 7$ & $3.05066 \mathrm{E}-003$ & $1.22517 \mathrm{E}-003$ & 4488 \\
\hline
\end{tabular}

Tabela 5.12: Erro e número de iterações em malha composta com sete niveis.

A seguir, apresentamos resultados para casos em que $h \neq 0$ e $\omega=\omega(x, y)$. O "salto de propriedade" tem tamanho $h$ e a transição depende de $d$. Com $h=10^{3}$ e $d=2$, os valores do erro e a ordem de convergência com a malha composta por dois níveis de refinamento estão na Tabela 5.13. Novamente verificamos a ordem de convergência esperada e comparando ao caso simples, em que $h=0$ e $\omega$ era constante na malha composta Tabela 5.9, vemos que o número de iterações para obtermos a solução do sistema é maior neste caso.

\begin{tabular}{cccccc}
\hline Malha & $\|e\|_{\infty}$ & $\mathrm{R}$ & $\|e\|_{2}$ & $\mathrm{R}$ & Iter \\
\hline $64 \times 64 L 2$ & $4.55936 \mathrm{E}-003$ & & $9.27781 \mathrm{E}-004$ & & 325 \\
$128 \times 128 L 2$ & $1.16399 \mathrm{E}-003$ & 3.917 & $2.34347 \mathrm{E}-004$ & 3.959 & 738 \\
$256 \times 256 L 2$ & $2.92955 \mathrm{E}-004$ & 3.973 & $5.88132 \mathrm{E}-005$ & 3.985 & 1806 \\
$512 \times 512 L 2$ & $7.34638 \mathrm{E}-005$ & 3.988 & $1.47263 \mathrm{E}-005$ & 3.994 & 5337 \\
\hline
\end{tabular}

Tabela 5.13: Erro, ordem de convergência e número de iteraçôes com $h=10^{3}, d=2$ e dois níveis de refinamento.

\section{Equação elíptica para variáveis centradas: condições de contorno arbitrárias}

Apresentamos a seguir a comparação entre as soluções obtidas ao usarmos a discretização matricial, usando a biblioteca PETSc, e a aquela obtida nível-a-nível e retalho-a-retalho usando o método multinível-multigrid, originalmente implementado no AMR2D [111]. No que segue, usamos uma discretização do operador diferencial baseada em fluxos tendo em vista problemas oriundos da condição de compatibilidade em malhas compostas SAMR (isto é, a validade do Teorema de Gauss em sua forma discreta em malhas compostas). Esta discretização permite o uso de condições de contorno de Neumann ou periódicas puras (ou uma combinação destas).

Para obter segunda ordem ao discretizarmos equações elípticas para variáveis centradas e condição de contorno arbitrária, alguns cuidados precisam ser tomados nas regiões que contêm as interfaces entre as malhas finas e grossas de forma a se obter a precisão desejada [64, 111]. O operador divergente deve satisfazer a seguinte identidade, advinda do Teorema de Gauss [96],

$$
\begin{aligned}
\int_{\Omega} \nabla \cdot \mathbf{f} & =\int_{\delta \Omega} \mathbf{f} \cdot \mathbf{n}, \\
h_{l}^{2} \sum_{i, j}(D \cdot \mathbf{f})_{i j} & =h_{l} \sum_{m}(\mathbf{f} \cdot \mathbf{n})_{m},
\end{aligned}
$$

onde $D$ é o operador divergente discretizado, $h$ é o espaçamento da malha (por simplicidade, 
tomamos aqui $h=\Delta x=\Delta y)$ e o índice $l$ denota o nível em análise, $\mathbf{f}=\left(f_{x}, f_{y}\right)$ é o fluxo a partir de uma variável centrada (e.g. $\varphi$ ), os índices $i$ e $j$ denotam as células pertencentes ao domínio $\Omega$ e o índice $m$ denota as células que estão no contorno do volume de controle.

Não é difícil ver que, para que a igualdade (5.13) seja satisfeita a nível discreto, nas interfaces fina-grossa (Figura 5.8), ao discretizarmos (5.13), devemos ter

$$
f_{x_{I+\frac{1}{2}, J}}=\frac{f_{x_{i-\frac{1}{2}, j}}+f_{x_{i-\frac{1}{2}, j+1}}}{2} .
$$

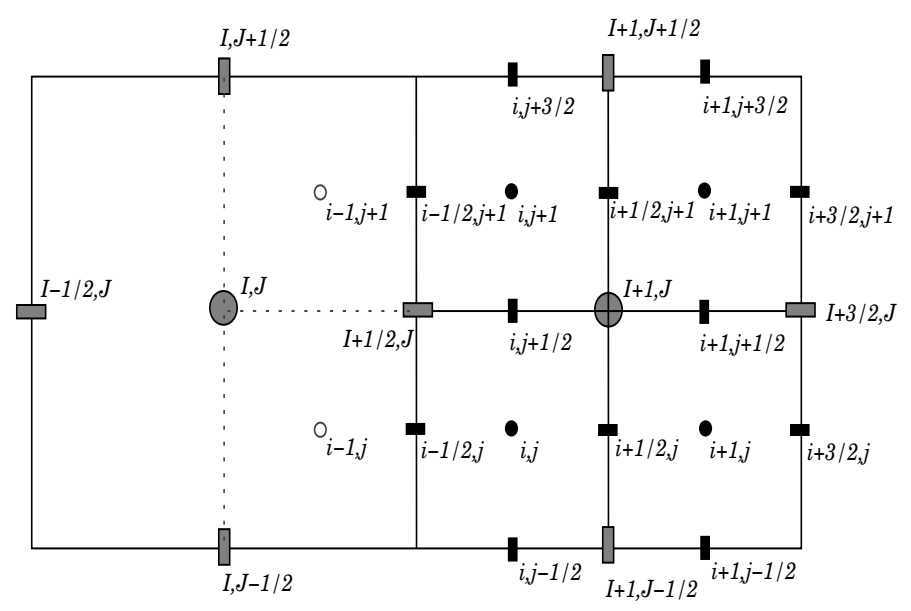

Figura 5.8: Interface fina-grossa em malha composta.

Longe de interfaces fina-grossas, o operador laplaciano continua sendo definido com a discretização usual de cinco pontos como em (5.10) (para o caso $\omega$ constante). Nas interfaces fina-grossas é necessário que seja feita uma "correção de fluxos". A definição do operador laplaciano em malhas compostas segue uma estratégia conservativa baseada na diferença de fluxos na qual empregamos um volume de controle ao redor de cada célula. Nesse contexto, a discretização para $\nabla^{2} \varphi=\nabla \cdot \mathbf{f}$, tal que $\mathbf{f}=\nabla \varphi$, em qualquer nível de refinamento é

$$
\begin{aligned}
\left(\nabla^{2} \varphi\right)_{i, j} & =(\nabla \cdot \mathbf{f})_{i j}, \\
& =\frac{f_{x_{i+\frac{1}{2}, j}}-f_{x_{i-\frac{1}{2}, j}}+f_{y_{i, j+\frac{1}{2}}}-f_{y_{i, j-\frac{1}{2}}}}{h},
\end{aligned}
$$

onde

$$
\begin{aligned}
\mathbf{f} & =\nabla \varphi, \\
f_{x_{i+\frac{1}{2}, j}} & =\frac{1}{h}\left(\varphi_{i+1, j}-\varphi_{i, j}\right), \\
f_{x_{i-\frac{1}{2}, j}} & =\frac{1}{h}\left(\varphi_{i, j}-\varphi_{i-1, j}\right), \\
f_{y_{i, j+\frac{1}{2}}} & =\frac{1}{h}\left(\varphi_{i, j+1}-\varphi_{i, j}\right), \\
f_{y_{i, j-\frac{1}{2}}} & =\frac{1}{h}\left(\varphi_{i, j}-\varphi_{i, j-1}\right),
\end{aligned}
$$

tal que $i$ e $j$ representam uma célula qualquer da malha composta.

$\mathrm{Na}$ interface fina-grossa, com o volume de controle ao redor de uma célula pertencente à malha 
grossa adjacente à interface fina-grossa, ao somar os fluxos dessa interface, temos que a discretização para esta região seguindo a Figura 5.8 é da forma

$$
\nabla^{2} \varphi_{I, J}=\frac{f_{x_{I+\frac{1}{2}, J}}^{* l}-f_{x_{I-\frac{1}{2}, J}}^{l}+f_{y_{I, J+\frac{1}{2}}}^{l}-f_{y_{I, J-\frac{1}{2}}}^{l}}{h_{l}},
$$

com

$$
f_{x_{I+\frac{1}{2}, J}^{* l}}^{* l}=\frac{1}{2}\left(f_{x_{i-\frac{1}{2}, j+1}}^{l+1}+f_{x_{i-\frac{1}{2}, j}}^{l+1}\right)
$$

$\mathrm{e}$

$$
\begin{array}{r}
f_{x_{i-\frac{1}{2}, j+1}}^{l+1}=\frac{\varphi_{i-1, j+1}^{l+1}-\varphi_{i, j+1}^{l+1}}{h_{l+1}}, \\
f_{x_{i-\frac{1}{2}, j}}^{l+1}=\frac{\varphi_{i-1, j}^{l+1}-\varphi_{i, j}^{l+1}}{h_{l+1}},
\end{array}
$$

onde os índices $I$ e $J$ identificam as células pertencentes ao retalho grosso e $i$ e $j$ são os índices das células localizadas no retalho mais fino, como mostramos na Figura 5.8.

Destacamos que $f_{x}$ e $f_{y}$ estão nas arestas vertical e horizontal das células e a discretização matricial que representa este operador deve respeitar isto. Também devemos notar que $\varphi_{i-1, j}^{l+1}$ e $\varphi_{i-1, j+1}^{l+1}$ são valores em células fantasmas e, portanto, vêm de uma interpolação fina-grossa. Assumindo que a porção de malha na Figura 5.8 não toca nenhuma fronteira do domínio, podemos dizer que a interpolação de $\varphi_{i-1, j}^{l+1}$ e $\varphi_{i-1, j+1}^{l+1}$ é da forma

$$
\begin{gathered}
\varphi_{i-1, j}^{l+1}=\omega_{I, J-1}^{1} \varphi_{I, J-1}^{l}+\omega_{I, J}^{1} \varphi_{I, J}^{l}+\omega_{I, J+1}^{1} \varphi_{I, J+1}^{l}+\omega_{i, j}^{1} \varphi_{i, j}^{l+1}+\omega_{i+1, j}^{1} \varphi_{i+1, j}^{l+1}, \\
\varphi_{i-1, j+1}^{l+1}=\omega_{I, J-1}^{2} \varphi_{I, J-1}^{l}+\omega_{I, J}^{2} \varphi_{I, J}^{l}+\omega_{I, J+1}^{2} \varphi_{I, J+1}^{l}+\omega_{i, j+1}^{2} \varphi_{i, j+1}^{l+1}+\omega_{i+1, j+1}^{2} \varphi_{i+1, j+1}^{l+1} .
\end{gathered}
$$

Para ilustrar, os elementos de uma linha da matriz que representa o operador laplaciano na interface fina-grossa, seguindo a equação (5.17), poderiam ser substituídos pelas representações obtidas para cada um dos elementos da equação (5.18)-(5.20) e (5.21)- (5.22), obtendo, ao separar cada uma das componentes, uma representação da forma

$$
\begin{aligned}
\nabla^{2} \varphi_{I, J} & =\omega_{i, j} \varphi_{i, j}^{l+1}+\omega_{i, j+1} \varphi_{i, j+1}^{l+1}+\omega_{i+1, j} \varphi_{i+1, j}^{l+1}+\omega_{i+1, j+1} \varphi_{i+1, j+1}^{l+1}+ \\
& +\omega_{I-1, J} \varphi_{I, J}^{l}+\omega_{I, J-1} \varphi_{I, J-1}^{l}+\omega_{I, J} \varphi_{I, J}^{l}+\omega_{I, J+1} \varphi_{I, J+1}^{l},
\end{aligned}
$$

onde cada valor dos pesos $\omega$ provém do correto agrupamento dos valores correspondentes a cada termo depois da substituição.

Para verificar a solução de $\nabla \cdot(\omega(x, y) \nabla \phi(x, y))=f(x, y)$ com a discretização matricial usando fluxos, usamos as funções

$$
\begin{gathered}
\phi(x, y)=\frac{\cos (2 \pi x) \sin (2 \pi y)}{2 \pi} \\
f(x, y)=\omega \nabla^{2} \varphi+\partial_{x} \omega \partial_{x} \varphi+\partial_{y} \omega \partial_{y} \varphi \quad \mathrm{e} \\
\omega(x, y)=(1+h)-h * \tanh \left[d *\left(\sqrt{\left(x-x_{c}\right)^{2}+\left(y-y_{c}\right)^{2}}-r_{c}\right)\right] .
\end{gathered}
$$

A tolerância usada é $10^{-8}$ e a biblioteca PETSc usa por default, novamente, o précondicionador 
ILU(0). A condição de contorno usada é periódica em todas as direções. Também usamos para definir $\omega$ os valores das variáveis $r_{c}=0.2, x_{c}=0.7, y_{c}=0.35$ e $d=180$. Um gráfico de $\omega(x, y)$ para o caso $h=10$ está na Figura 5.9. Este valor de $d$ é tal que o nível com espaçamento $1 / 256$ tem a transição em 4 células computacionais desse nível, como mostramos na Figura 5.9(b).
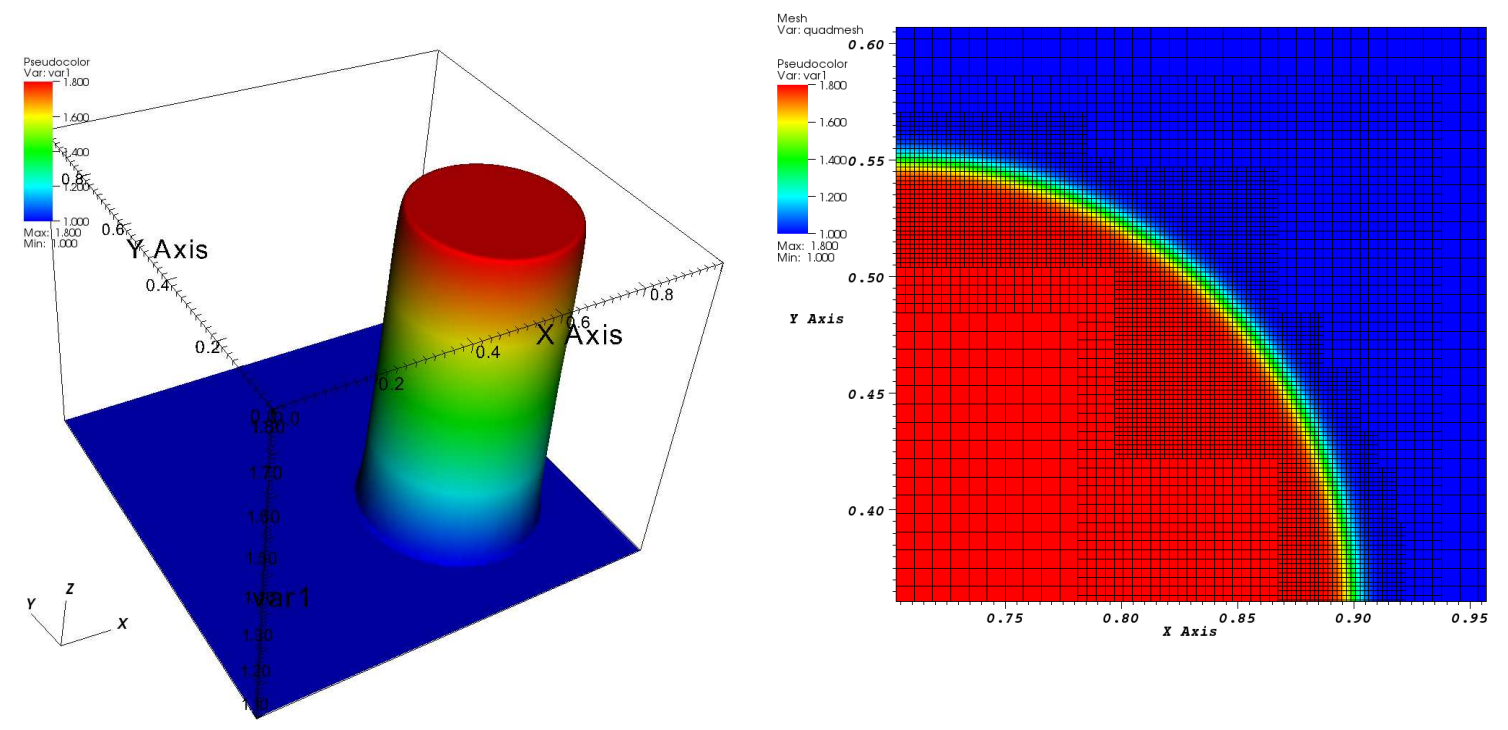

Figura 5.9: $\omega$ variável. (a) Altura e (b) zona de transição.

As funções e as malhas escolhidas neste teste permitem que comparemos os resultados diretamente com aqueles reportados por Villar [111]. Empregamos as mesmas malhas, mostradas na Figura 5.10, para o caso com malha uniforme e com malha composta.
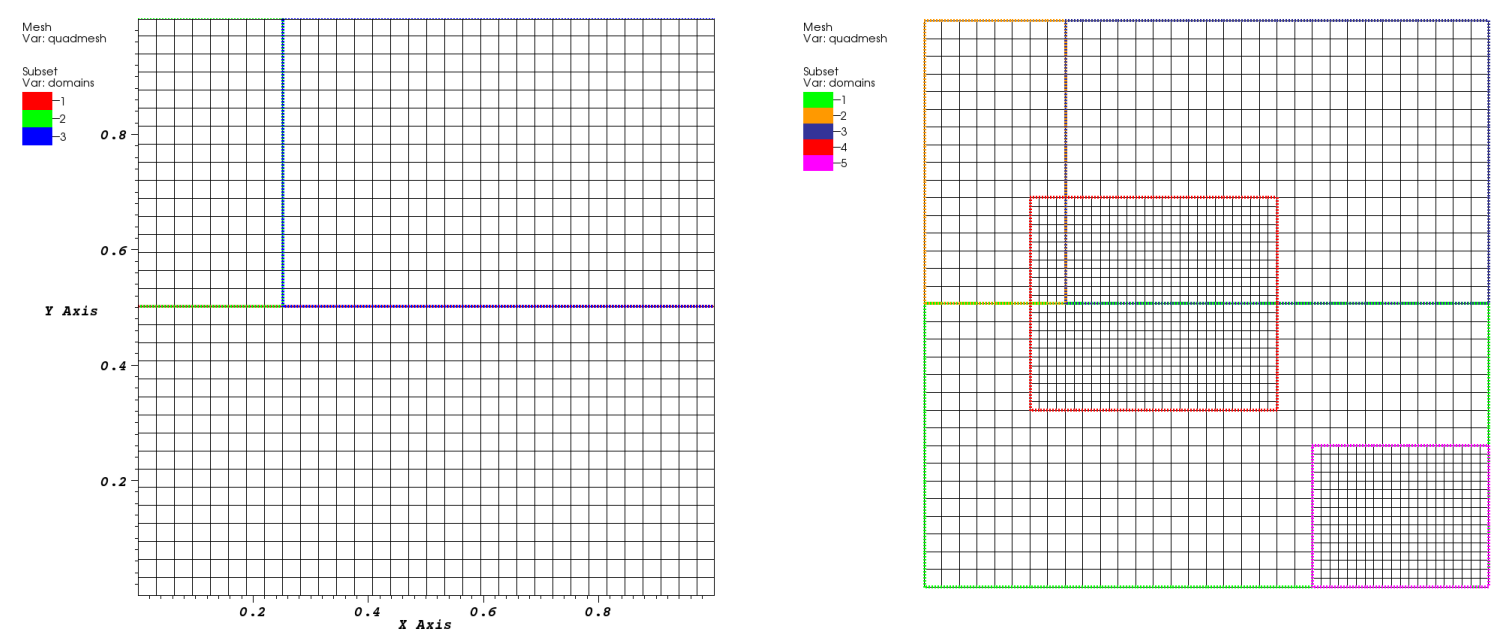

Figura 5.10: Malhas (a) uniforme $32 \times 32 L 1=$ G5L1 e (b) composta G5L2, com nivel base $32 \times 32$ e dois niveis de refinamento.

O primeiro teste foi efetuado em uma malha uniforme, porém formada por uma união de retalhos, a qual mostramos na Figura 5.10(a). A malha inicial que usamos G5L1, tem $32 \times 32$ células computacionais. Para esta malha, o número total de células é 1.260, incluindo as células fantasmas 
contidas em todos os retalhos. O número de células visíveis é 1.024, como era de se esperar por ser uma malha uniforme. Nas comparações empregamos a norma L2 para malhas compostas definida na Seção 5.1.

Neste primeiro teste, definimos $\omega$ constante e $h=0$ e os resultados obtidos estão na Tabela 5.14. Da biblioteca PETSc, empregamos os métodos BCGS (Método do Gradiente Bi-conjungado Estabilizado) e o GMRES, ambos com o précondicionador default ILU(0). Também usamos o método multinível-multigrid (MMG) do AMR2D. Podemos observar que o estudo de convergência aponta segunda ordem de precisão, como esperado.

\begin{tabular}{cccc}
\hline Solver & Malha & $\|e\|_{2}$ & $r_{2}$ \\
\hline \multirow{4}{*}{ MMG } & G5L1 & $2.5615 \mathrm{E}-04$ & \\
& G6L1 & $6.3946 \mathrm{E}-05$ & 4.0058 \\
& G7L1 & $1.5980 \mathrm{E}-05$ & 4.0014 \\
& G8L1 & $3.9948 \mathrm{E}-06$ & 4.0004 \\
& G9L1 & $9.9868 \mathrm{E}-07$ & 4.0001 \\
\hline \multirow{5}{*}{ GMRES } & G5L1 & $2.5615 \mathrm{E}-04$ & \\
& G6L1 & $6.3946 \mathrm{E}-05$ & 4.0058 \\
& G7L1 & $1.5981 \mathrm{E}-05$ & 4.0014 \\
& G8L1 & $3.9951 \mathrm{E}-06$ & 4.0002 \\
& G9L1 & $1.0008 \mathrm{E}-06$ & 3.9919 \\
\hline \multirow{5}{*}{ BCGS } & G5L1 & $2.5615 \mathrm{E}-04$ & \\
& G6L1 & $6.3946 \mathrm{E}-05$ & 4.0058 \\
& G7L1 & $1.5980 \mathrm{E}-005$ & 4.0015 \\
& G8L1 & $3.9948 \mathrm{E}-06$ & 4.0003 \\
& G9L1 & $9.9985 \mathrm{E}-07$ & 3.9954 \\
\hline
\end{tabular}

\begin{tabular}{cccc}
\hline Solver & Malha & $\|e\|_{2}$ & $r_{2}$ \\
\hline \multirow{4}{*}{ MMG } & G5L2 & $2.2673 \mathrm{E}-04$ & \\
& G6L2 & $5.6298 \mathrm{E}-05$ & 4.0275 \\
& G7L2 & $1.4035 \mathrm{E}-05$ & 4.0110 \\
& G8L2 & $3.5047 \mathrm{E}-06$ & 4.0048 \\
& G9L2 & $8.7568 \mathrm{E}-07$ & 4.0023 \\
\hline \multirow{5}{*}{ GMRES } & G5L2 & $2.2673 \mathrm{E}-05$ & \\
& G6L2 & $5.6297 \mathrm{E}-05$ & 4.0275 \\
& G7L2 & $1.4036 \mathrm{E}-05$ & 4.0109 \\
& G8L2 & $3.5066 \mathrm{E}-06$ & 4.0028 \\
& G9L2 & $8.8945 \mathrm{E}-07$ & 3.9424 \\
\hline \multirow{5}{*}{ BCGS } & G5L2 & $2.26739 \mathrm{E}-04$ & \\
& G6L2 & $5.62985 \mathrm{E}-05$ & 4.0274 \\
& G7L2 & $1.40362 \mathrm{E}-05$ & 4.0109 \\
& G8L2 & $3.50682 \mathrm{E}-06$ & 4.0025 \\
& G9L2 & $8.82605 \mathrm{E}-07$ & 3.9733 \\
\hline
\end{tabular}

Tabela 5.14: Erro e ordem de convergência com $h=0$ em malha uniforme (à esquerda) e em malha composta (à direita), usando condição de contorno periódica em todas as direções.

Nas mesmas condições, mas agora para a malha da Figura 5.10(b), com um nível a mais, temos os valores de erro e de ordem de convergência na Tabela 5.14. Para esta malha temos que o número total de células é de 2.436 e o número total de células visíveis é de 1.768 . Novamente, obtivemos a ordem de convergência esperada e o uso da malha composta não degradou a aproximação quando comparados os resultados com aqueles obtidos na malha uniforme.

Por último, temos um teste no qual usamos uma malha composta que segue a zona de transição da variável $\omega(x, y)$ quando $h \neq 0$, como mostramos na Figura 5.11, onde temos a representação gráfica da malha com seus respectivos retalhos e os valores de $w(x, y)$ para $h=0.4$. A malha inicial G7L3 tem malha base de $128 \times 128$ células computacionais e três níveis de refinamento. Esta malha tem 77.820 células computacionais, 45.118 células visíveis e 20 retalhos. A ideia nesse teste é variar o valor de $h$ para verificar a robustez dos métodos da biblioteca PETSc em termos de convergência. Pretendemos também explorar o uso e verificar o comportamento de seus pré;condicionadores, além de registrar alguns resultados iniciais explorando o paralelismo.

Com o uso da malha da Figura 5.11, o primeiro teste realizado foi com $h=0$. Neste caso, o valor do erro e a ordem de convergência estão na Tabela 5.15. Novamente obtivemos a ordem de convergência esperada. A precisão do erro diminuiu ao compararmos com os valores obtidos com a malha uniforme na Tabela 5.14.

Continuando com a malha composta da Figura 5.11, mas agora sem o estudo de convergência, 

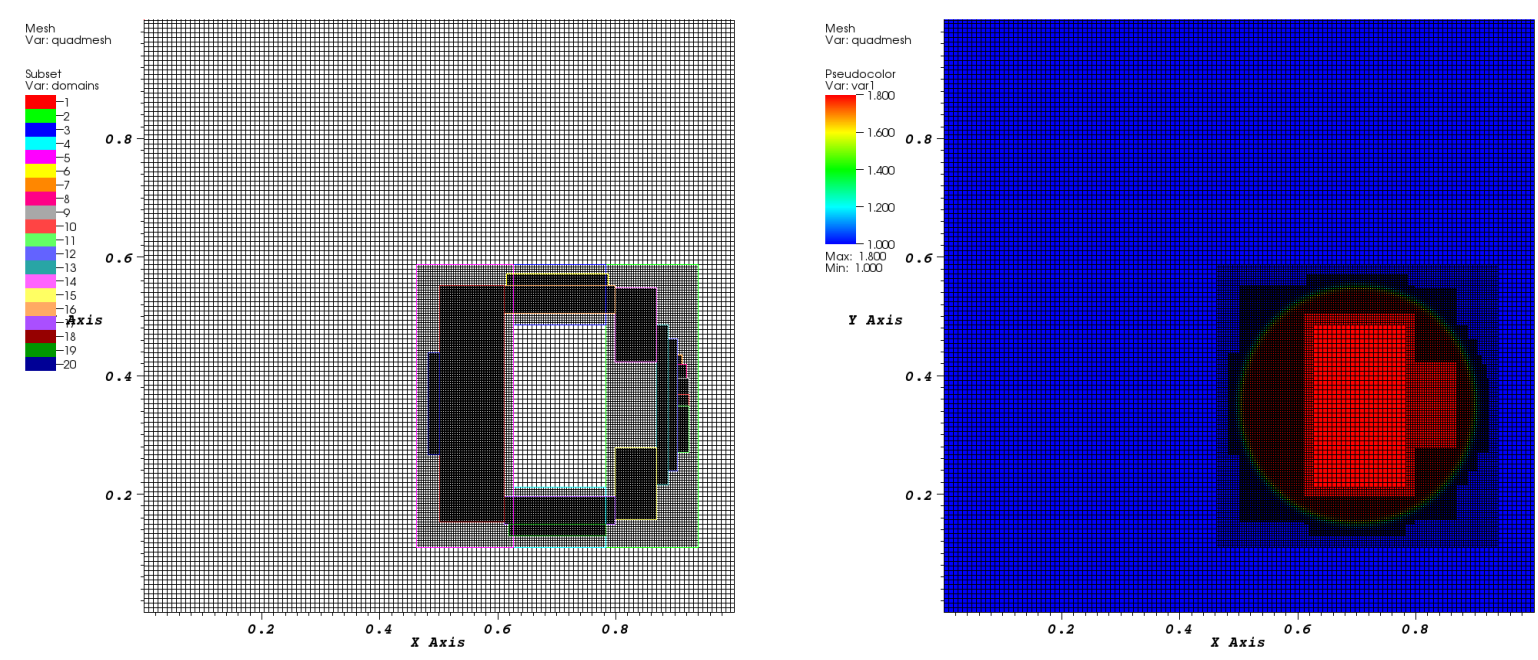

Figura 5.11: Malha composta $128 \times 128 L 2=G 7 L 3$ e $\omega(x, y)$ para $h=0.4$.

\begin{tabular}{cccc}
\hline Solver & Malha & $\|e\|_{2}$ & $r_{2}$ \\
\hline \multirow{4}{*}{ MMG } & G7L3 & $1.4038 \mathrm{E}-005$ & \\
& G8L3 & $3.5052 \mathrm{E}-006$ & 4.0049 \\
& G9L3 & $8.7581 \mathrm{E}-007$ & 4.0023 \\
& G10L3 & $2.1888 \mathrm{E}-007$ & 4.0012 \\
\hline \multirow{4}{*}{ GMRES } & G7L3 & $1.4039 \mathrm{E}-005$ & \\
& G8L3 & $3.5042 \mathrm{E}-006$ & 4.0065 \\
& G9L3 & $8.8463 \mathrm{E}-007$ & 3.9612 \\
& G10L3 & $2.2947 \mathrm{E}-07$ & 3.8551 \\
\hline \multirow{5}{*}{ BCGS } & G7L3 & $1.4039 \mathrm{E}-005$ & \\
& G8L3 & $3.5080 \mathrm{E}-006$ & 4.0021 \\
& G9L3 & $8.8397 \mathrm{E}-007$ & 3.9685 \\
& G10L3 & $2.2947 \mathrm{E}-007$ & 3.8522 \\
\hline
\end{tabular}

Tabela 5.15: Erro e ordem de convergência com $h=0$ em malha composta. 
solucionamos este mesmo caso usando diferentes métodos da biblioteca PETSc. Mostramos na Tabela 5.16 os resultados obtidos ${ }^{4}$. Dentre os métodos empregados na resolução do sistema linear, apenas o GMRES e o BCGS convergiram. Uma outra observação importante é que entre o GMRES e o BCGS, o BCGS precisa de um tempo bem menor para resolver o sistema linear ("t_solve"). Entretanto, nenhum deles para este caso compete com o método multinível-multigrid que, com apenas seis V-ciclos, obtém a convergência na precisão desejada.

\begin{tabular}{ccccc}
\hline Solver & Result & $\|e\|_{2}$ & Iter & t_solve (s) \\
\hline GMRES & Converg & $8.8463 \mathrm{E}-07$ & 3136 & 308.96 \\
BCGS & Converg & $8.8397 \mathrm{E}-07$ & 665 & 63.96 \\
BiCG & Diverg & 1.0502627 & 2952 & 174.39 \\
CGS & Diverg & 0.4069322 & 21 & 2.48 \\
lsqr & Diverg & $7.9577 \mathrm{E}-002$ & 0 & 0.39 \\
chebychev & Diverg & $2.24578 \mathrm{E}-02$ & 10000 & 490.88 \\
richardson & Diverg & $2.24722 \mathrm{E}-02$ & 10000 & 467.61 \\
MMG & Converg & $8.7581 \mathrm{E}-07$ & 6 & 6.98 \\
\hline
\end{tabular}

Tabela 5.16: Comparação entre métodos do PETCs e o método multinivel-multigrid "nativo" do AMR2D $(h=0$ em malha composta).

Dado que o método BCGS da biblioteca PETSc foi o mais rápido para determinar a solução, experimentamos com ele diferentes pré-condicionadores, lembrando que até aqui usamos sempre o précondicionador ILU(0). Testamos quatro pré-condicionadores diferentes e um caso sem précondicionador, cujos resultados mostramos na Tabela 5.16. Os pré-condicionadores ILU(0) e bjacobi 5 foram os que realizaram o tempo computacional mantendo uma boa precisão na solução. Por outro lado, quando não usamos pré-condicionador o tempo de computo aumentou significativamente e a precisão obtida diminuiu.

\begin{tabular}{cccc}
\hline Precondicionador & $\|e\|_{2}$ & Iter & t_solve(s) \\
\hline ilu & $8.8397 \mathrm{E}-07$ & 665 & 60.14 \\
bjacobi & $8.8397 \mathrm{E}-07$ & 665 & 62.74 \\
jacobi & $1.2468 \mathrm{E}-06$ & 2342 & 131.88 \\
sor & $8.9050 \mathrm{E}-07$ & 904 & 90.74 \\
none & $3.7029 \mathrm{E}-04$ & 6056 & 324.77 \\
\hline
\end{tabular}

Tabela 5.17: Comparação de pré-condicionadores do PETCs fixando o solver BCGS $(h=0$ em malha composta).

Investigamos brevemente o paralelismo da biblioteca PETSc empregando a malha G9L3, obtida ao se refinar a malha da Figura 5.11. Escolhemos esta malha porque com ela se tinha um tempo de cômputo considerável no calculo da solução do sistema nas contas da Tabela 5.15. Usamos 1, 2 e 4 processadores com os métodos GMRES e BCGS, com précondicionador ILU(0). O método multinível-multigrid é serial e só pode ser comparado com os outros métodos quando usamos um processador. Mostramos os resultados obtidos na Tabela 5.18 e vemos que, quando aumentamos o número de processadores, o tempo de cômputo cai. Entretanto, ainda é muito maior àquele obtido

\footnotetext{
${ }^{4}$ No PETSc as siglas "bicg", "cgs" e "lsqr" correspondem aos métodos dos gradientes bi-conjugados, gradientes conjugados quadrados e mínimos quadrados para sistemas esparsos, respectivamente.

${ }^{5}$ A sigla "bjacobi" correspondem ao précondicionar Jacobi por blocos.
} 
com o método multinível-multigrid. Também podemos ver que a precisão da solução continua a mesma e que, quando aumentamos o número de processadores a serem usados, a quantidade de iterações tanto do GMRES como do BCGS também aumentam. Como o PETSc é uma biblioteca externa ao AMR2D, incluímos nesta tabela o tempo gasto para passar a solução do PETSc que se encontra distribuída nos diferentes processadores para a variável serial do AMR2D ("t_PETScAMR"), respeitando a estrutura de dados e as respetivas posições da variável no AMR2D.

\begin{tabular}{cccccc}
\hline np & solver & $\|e\|_{2}$ & Iter & t_solve(s) & t_PETScAMR(s) \\
\hline 1 & GMRES & $8.8463 \mathrm{E}-07$ & 3136 & 219.48 & $7.918 \mathrm{E}-03$ \\
2 & GMRES & $8.8471 \mathrm{E}-07$ & 3677 & 150.91 & $1.022 \mathrm{E}-02$ \\
4 & GMRES & $8.8417 \mathrm{E}-07$ & 2959 & 87.22 & $1.335 \mathrm{E}-02$ \\
1 & BCGS & $8.8397 \mathrm{E}-07$ & 665 & 60.14 & $7.957 \mathrm{E}-03$ \\
2 & BCGS & $8.81409 \mathrm{E}-07$ & 740 & 39.62 & $9.848 \mathrm{E}-03$ \\
4 & BCGS & $8.8973 \mathrm{E}-07$ & 782 & 25.19 & $1.452 \mathrm{E}-02$ \\
1 & MMG & $8.7580 \mathrm{E}-07$ & 6 & 6.985 & - \\
\hline
\end{tabular}

Tabela 5.18: Comparação em paralelo entre os métodos GMRES e BCGS do PETSc e o método multinivelmultigrid "nativo" do AMR2D.

A seguir, reportamos o comportamento, tanto do Método BCGS da biblioteca PETSc quanto o do método multinível-multigrid "nativo" no AMR2D, na solução da equação elíptica com as mesmas funções exatas, com a malha G9L3 escolhida e diferentes valores $h$ do tamanho do salto. Escolhemos o BCGS por que foi o método do PETSc que gastou menos tempo para determinar a solução do sistema.

Na Tabela 5.19, mostramos os valores obtidos para $h=0.1$ usando paralelismo e o précondicionador ILU(0). Podemos ver que a quantidade de iterações do método multinível-multigrid aumentou ao compararmos ao que ocorreu com $h=0$ e reportado na Tabela 5.18.

\begin{tabular}{cccccc}
\hline $\mathrm{np}$ & solver & $\|e\|_{2}$ & Iter & t_solve(s) & t_PETScAMR(s) \\
\hline 1 & BCGS & $3.7035 \mathrm{E}-004$ & 664 & 59.93 & $7.8620 \mathrm{E}-003$ \\
2 & BCGS & $3.7036 \mathrm{E}-004$ & 732 & 39.09 & $9.6909 \mathrm{E}-003$ \\
4 & BCGS & $3.7029 \mathrm{E}-004$ & 761 & 24.17 & $1.4565 \mathrm{E}-002$ \\
1 & MMG & $3.702 \mathrm{E}-004$ & 13 & 15.49 & - \\
\hline
\end{tabular}

Tabela 5.19: Comparações de paralelismo ( $h=0.1$ em malha composta).

Na Tabela 5.20, temos os valores obtidos para $h=0.4$. Podemos ver que a quantidade de iterações do método multinível-multigrid "nativo" aumentou consideravelmente, mesmo ao incrementarmos o número de relaxações do Gauss-Seidel preto-vermelho e empregarmos o SIP-solver como relaxador adicional (Strongly-Implicit-Procedure [38]), disponível no AMR2D. Por outro lado, o BCGS teve tempo menor ao do método multinível-multigrid, mesmo com um processador só. Ao aumentar o número de processos, o tempo tomado pelo método BCGS diminui e por isto, neste caso, achamos interessante fazer um pequeno estudo de escalas de paralelismo - muito embora este estudo seja mais apropriado quando temos uma quantidade maior de processadores. Investigamos o speedup, a eficiência e o trabalho. Disponibilizamos estes resultados na Tabela 5.20. Incluímos também uma estimativa do tempo por iteração $(t \times$ iter $)$ tomado pelo método numérico empregado em cada caso. 


\begin{tabular}{|c|c|c|c|c|c|}
\hline $\mathrm{np}$ & solver & $\|e\|_{2}$ & Iter & t_solve(s) & t_PETScAMR(s) \\
\hline 1 & BCGS & $1.4230 \mathrm{E}-003$ & 766 & 68.83 & $7.9061 \mathrm{E}-003$ \\
\hline 2 & BCGS & $1.4231 \mathrm{E}-003$ & 785 & 41.85 & $9.6888 \mathrm{E}-003$ \\
\hline 4 & BCGS & $1.4232 \mathrm{E}-003$ & 796 & 25.32 & $1.4554 \mathrm{E}-002$ \\
\hline 1 & MMG & $1.4231 \mathrm{E}-003$ & 90 & 120.80 & - \\
\hline 1 & $\mathrm{MMG}+\mathrm{SIP}$ & $1.4231 \mathrm{E}-003$ & 62 & 91.62 & - \\
\hline & solver & $t \times \operatorname{iter}(\mathrm{s})$ & speedur & Eficiência & Trabalho \\
\hline & BCGS & 0.090 & 1.00 & 1.00 & 68.83 \\
\hline & BCGS & 0.053 & 1.64 & 0.82 & 83.70 \\
\hline & BCGS & 0.032 & 2.72 & 0.68 & 101.26 \\
\hline
\end{tabular}

Tabela 5.20: Comparações de paralelismo ( $h=0.4$ em malha composta).

Mostramos na Figura 5.12 gráficos com o comportamento dos resíduos para cada iteração do método multinível-multigrid e do BCGS para $h=0.1$ e para $h=0.4$. Podemos ver como o resíduo com o MMG cai mais acentuadamente com menor número de iterações comparado com o resíduo que teve o método BCGS. Os resíduos por iteração com o BCGS oscilam um pouco, mas sempre caem para a tolerância desejada.
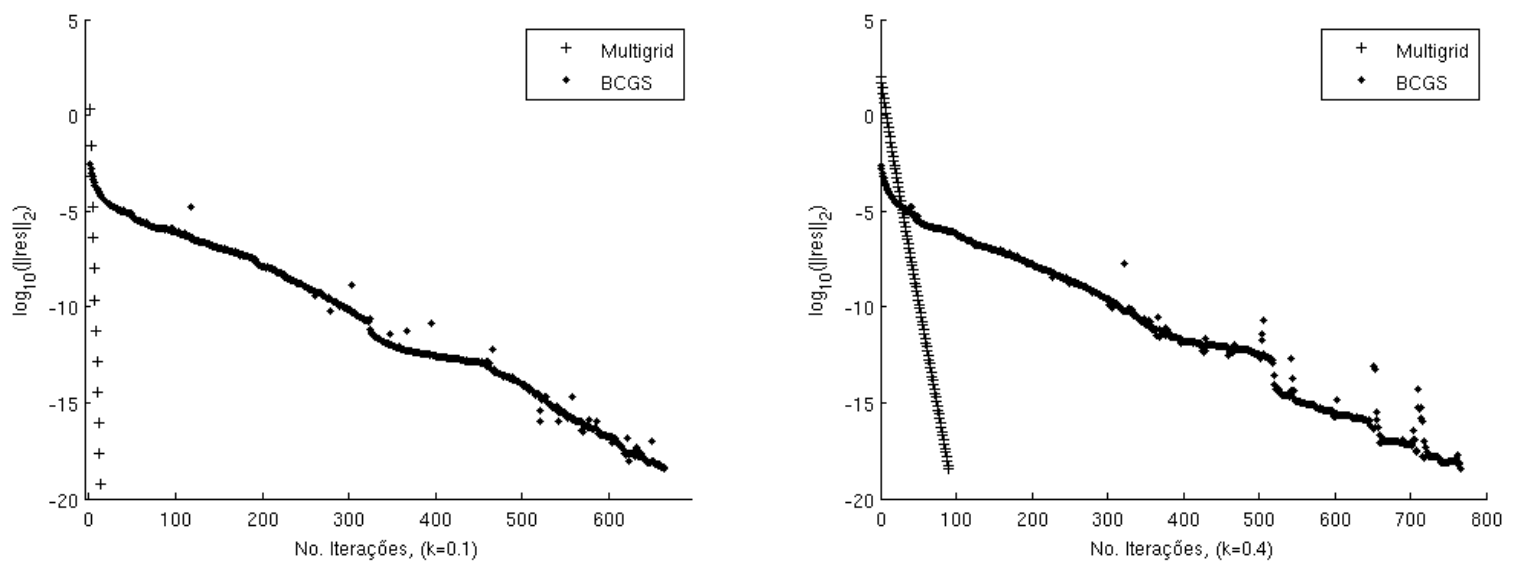

Figura 5.12: Comportamento do resíduo por iteração com os métodos BCGS e o multinível-multigrid para $h=0.1$ (esquerda) e para $h=0.4$ (direita).

Ao testarmos para $h>0.5$, obtivemos a divergência do método multinível-multigrid. Para diferentes valores de $h$, comparamos então a solução via BCGS da biblioteca PETSc. Os resultados encontrados estão na Tabela 5.21. Notamos que, quando aumentamos $h$, o erro também aumenta e, portanto, precisaríamos usar malhas mais finas para obter um erro menor.

\begin{tabular}{cccc}
\hline$h$ & $\|e\|_{\infty}$ & $\|e\|_{2}$ & Iter \\
\hline 1 & $1.0214 \mathrm{E}-02$ & $3.4243 \mathrm{E}-03$ & 667 \\
10 & $6.6692 \mathrm{E}-02$ & $3.2319 \mathrm{E}-02$ & 818 \\
100 & $5.6406 \mathrm{E}-01$ & $3.1282 \mathrm{E}-01$ & 738 \\
1000 & $5.2353 \mathrm{E}+00$ & $3.0277 \mathrm{E}+00$ & 621 \\
\hline
\end{tabular}

Tabela 5.21: Diferentes valores de h para o BCGS em malha composta.

Nos testes anteriores, a função $\omega$ pode ser interpretada como em um teste de "gotículas" no 
qual a massa específica exterior é menor que a interior. A seguir, tendo em mente o passo de correção da pressão nas equações de Navier-Stokes, definiremos $\omega(x, y)=1 / \rho(x, y)$, onde $\rho(x, y)=$ $1+h * \sin ^{2}(2 \pi x) \sin ^{2}(2 \pi y)$. Logo, se $\rho$ é grande então $\omega=1 / \rho$ é um valor pequeno.

Para diferentes métodos, temos os comportamentos obtidos reportados na Tabela 5.22. Verificamos que a ordem de convergência tende à segunda ordem, como esperávamos. O número de iterações com o método multinível-multigrid se mantém constante com o refinamento. Entretanto, o tempo de processamento vai aumentando. Os métodos GMRES e BCGS, neste caso, tomam um tempo bem maior quando comparados com o método multinível-multigrid e o número de iterações aumenta com o refinamento da malha. Para comparar com os resultados reportados por Villar [111], usamos novamente a malha composta de dois níveis dada na Figura 5.10(b).

\begin{tabular}{cccccc}
\hline Solver & Malha & $\|e\|_{2}$ & $r_{2}$ & iter & t_solver(s) \\
\hline MMG & G6L2 & $5.6298 \mathrm{E}-05$ & - & 6 & $3.9591 \mathrm{E}-02$ \\
& G7L2 & $1.4036 \mathrm{E}-05$ & 4.011 & 6 & $1.7456 \mathrm{E}-01$ \\
& G8L2 & $3.5047 \mathrm{E}-06$ & 4.005 & 6 & $7.6739 \mathrm{E}-01$ \\
& G9L2 & $8.7568 \mathrm{E}-07$ & 4.002 & 6 & $4.2753 \mathrm{E}+00$ \\
\hline GMRES & G6L2 & $5.6298 \mathrm{E}-05$ & - & 114 & $7.5015 \mathrm{E}-02$ \\
& G7L2 & $1.4036 \mathrm{E}-05$ & 4.011 & 288 & $7.6286 \mathrm{E}-01$ \\
& G8L2 & $3.5066 \mathrm{E}-06$ & 4.003 & 789 & $8.6052 \mathrm{E}+00$ \\
& G9L2 & $8.8945 \mathrm{E}-07$ & 3.942 & 2546 & $1.1798 \mathrm{E}+02$ \\
\hline BCGS & G6L2 & $5.6299 \mathrm{E}-05$ & - & 70 & $6.2365 \mathrm{E}-02$ \\
& G7L2 & $1.4036 \mathrm{E}-05$ & 4.011 & 131 & $4.5088 \mathrm{E}-01$ \\
& G8L2 & $3.5068 \mathrm{E}-06$ & 4.003 & 234 & $3.3129 \mathrm{E}+00$ \\
& G9L2 & $8.8287 \mathrm{E}-07$ & 3.972 & 437 & $2.5691 \mathrm{E}+01$ \\
\hline
\end{tabular}

Tabela 5.22: Solução manufaturada em malha composta com dois niveis de refinamento e $h=0.0$.

Para a nova definição de $\omega$, investigamos o que ocorre para maiores valores de $h$. O comportamento obtido pelo métodos GMRES, BCGS e multinível-multigrid são apresentados na Tabela 5.23, na qual GMRES só é usado uma vez porque o tempo de cômputo for tão mais alto que os demais para $h=0$ que não o empregamos mais para os demais valores de $h$. O método multinível-multigrid convergiu para todos os valores de $h$, mas o custo computacional aumentou com o aumento de $h$ e, quando comparado com o BCGS, ele deixa de ser útil.

\begin{tabular}{ccccc}
\hline$h$ & Solver & $\|e\|_{2}$ & Iter & t_solver \\
\hline 0 & MMG & $8.76 \mathrm{E}-007$ & 6 & 4.275 \\
0 & GMRES & $8.89 \mathrm{E}-007$ & 2546 & 117.982 \\
0 & BCGS & $8.83 \mathrm{E}-007$ & 437 & 25.69 \\
\hline 1 & MMG & $6.74 \mathrm{E}-007$ & 25 & 20.889 \\
1 & BCGS & $6.88 \mathrm{E}-007$ & 484 & 27.225 \\
\hline 100 & MMG & $2.03 \mathrm{E}-005$ & 512 & 437.206 \\
100 & BCGS & $2.04 \mathrm{E}-005$ & 586 & 33.932 \\
\hline 1000 & MMG & $7.99 \mathrm{E}-004$ & 1209 & 1033.832 \\
1000 & BCGS & $7.99 \mathrm{E}-004$ & 661 & 38.129 \\
\hline
\end{tabular}

Tabela 5.23: Diferentes valores de $h$ em malha composta.

Para finalizarmos esta subseção, apresentamos resultados que caracterizam a matriz associada à 
discretização da equação. Para $h=0$, temos o padrão de esparsidade na Figura 5.13 e informações sobre a matriz para os diferentes valores de $h$ na Tabela 5.24.

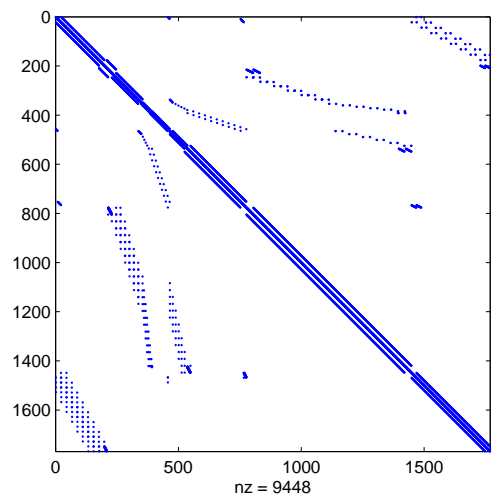

Figura 5.13: Padrão de esparsidade para $h=0$ correspondente à discretização na malha composta da Figura 5.10 .

Na Tabela 5.24, podemos ver que o valor máximo na diagonal tende a zero com o aumento de $h$, de onde se conclui que, tendo como relaxador o Método Gauss-Seidel, o uso do método multinívelmultigrid fica comprometido. Com o aumento de $h$, o mau condicionamento da matriz aumenta, como esperado.

\begin{tabular}{cccccc}
\hline$h$ & $\min (\operatorname{Diag}(A))$ & $\max (\operatorname{Diag}(A))$ & $\min \left(\lambda_{A}\right)$ & $\max \left(\lambda_{A}\right)$ & $\|A\|_{2}$ \\
\hline \hline 0 & $-1.6384 \mathrm{e}+04$ & $-4.0960 \mathrm{e}+04$ & $-3.2645 \mathrm{e}+04$ & $-3.2233 \mathrm{e}+04$ & 1.0128 \\
\hline 1 & $-1.6384 \mathrm{e}+04$ & $-2.0866 \mathrm{e}+03$ & $-3.2360 \mathrm{e}+04$ & $-3.1207 \mathrm{e}+04$ & 1.0369 \\
\hline 100 & $-1.6356 \mathrm{e}+04$ & $-4.2122 \mathrm{e}+01$ & $-3.0871 \mathrm{e}+04$ & $-2.6452 \mathrm{e}+04$ & 1.1671 \\
\hline 1000 & $-1.6111 \mathrm{e}+04$ & $-4.2516 \mathrm{e}+00$ & $-2.8720 \mathrm{e}+04$ & $-2.0205 \mathrm{e}+04$ & 1.4214 \\
\hline 10000 & $-1.4312 \mathrm{e}+04$ & $-4.2556 \mathrm{e}-01$ & $-2.4044 \mathrm{e}+04$ & $-1.2341 \mathrm{e}+04$ & 1.9483 \\
\hline
\end{tabular}

Tabela 5.24: Informações da matriz para $\omega=1 / \rho$ e diferentes valores de $h$.

\subsubsection{Segundo problema-modelo: equação elíptica para variáveis nas arestas}

O estudo deste problema é muito importante uma vez que esta é a forma matricial que usamos para a solução das equações de Stokes estacionárias (2.41)-(2.44) por meio do Método de Uzawa com BiCG-Stab apresentado no Capítulo 3.

Seja $\mathbf{u}=[u, v]^{T}$ um campo vetorial com as componentes $u$ e $v$ definidas no meio das arestas verticais e horizontais, respectivamente. Apresentamos nesta seção a solução da equação de Poisson (5.7) aplicada a variável $\mathbf{u}$, dada por

$$
\nabla \cdot(\omega(x, y) \nabla \mathbf{u}(x, y))=\mathbf{f}(x, y), \quad(x, y) \in \Omega
$$

onde $\mathbf{f}=\left[f_{1}, f_{2}\right]^{T}$ e um campo vetorial e $\omega$ é uma variável escalar.

Como descrevemos na seção anterior, a discretização na malha composta deve satisfazer a conservação de fluxos (5.13), especialmente quando temos condições de contorno de Neumann ou condições periódicas puras ou uma combinação delas. No caso de variáveis definidas no meio das arestas, para que a discretização da equação (5.25) cumpra a conservação de fluxos (5.13), a discretização deve 
satisfazer uma condição nas interfaces fina-grossa similar àquela dada para variáveis definidas no centro de célula em (5.14). Mas neste caso, nós não conhecemos esta condição. Por isto determinamos a solução numérica com condições de Dirichlet em todas as arestas do contorno ou qualquer combinação que tenha pelo menos uma aresta com condição de Dirichlet. Além disso, assumimos $\omega(x, y)=1$. Neste caso, usamos a discretização do operador laplaciano com a fórmula usual de cinco pontos. Assim, para $\left(\begin{array}{c}\nabla^{2} u \\ \nabla^{2} v\end{array}\right)=\left(\begin{array}{l}f_{1} \\ f_{2}\end{array}\right)$, a discretização é dada por

$$
\left(\begin{array}{l}
\frac{u_{i+1, j}^{n+1}-2 u_{i j}^{n+1}+u_{i-1, j}^{n+1}}{\Delta x^{2}}+\frac{u_{i, j+1}^{n+1}-2 u_{i j}^{n+1}+u_{i, j-1}^{n+1}}{\Delta y^{2}} \\
\frac{v_{i+1, j}^{n+1}-2 v_{i j}^{n+1}+v_{i-1, j}^{n+1}}{\Delta x^{2}}+\frac{v_{i, j+1}^{n+1}-2 v_{i j}^{n+1}+v_{i, j-1}^{n+1}}{\Delta y^{2}}
\end{array}\right)=\left(\begin{array}{c}
f_{1, i j} \\
f_{2, i j}
\end{array}\right) .
$$

A forma matricial é obtida ao agruparmos os termos de mesmos subíndices. Como a discretização (5.26) possui as variáveis $u$ e $v$ desacopladas, podemos criar uma matriz para cada uma delas. Desta forma resultam dois sistemas, um para $\nabla^{2} u=f_{1}$ e outro para $\nabla^{2} v=f_{2}$. Criamos uma estrutura matricial que representa o cálculo de células fantasmas para $u$ e outra para $v$. Depois criamos a matriz para a discretização (5.26), uma associada à variável $u$ e outra associada à variável $v$. Entretanto na biblioteca PETSc criamos uma única matriz $A$, onde primeiro bloco da matriz corresponde à discretização de $u$ e o segundo, à discretização de $v$. A enumeração para a variável $u$ no PETSc continua sendo a mesma. Por outro lado, a enumeração para a variável $v$ sofre uma pequena mudança ao somarmos a cada índice a dimensão da matriz do primeiro bloco. A ideia de fazer a solução deste problema de forma acoplada serve como experiência para as discretizações que serão acopladas.

Para o teste de verificação numérica, utilizamos as funções

$$
\begin{aligned}
& u(x, y)=\sin (2 \pi x) \cos (2 \pi y), \\
& v(x, y)=\exp [\sin (2 \pi x) \cos (2 \pi y)], \\
& \omega(x, y)=1,
\end{aligned}
$$

sendo $\mathbf{f}$ dada por $f_{1}(x, y)=\nabla^{2} u$ e $f_{2}(x, y)=\nabla^{2} v$.

A Figura 5.14 mostra a malha composta utilizada, G4L3. A malha G4L3 possui um nível base com $16 \times 16$ células e, além deste, dois níveis de refinamento. Além disso, a Figura 5.14 mostra a solução aproximada obtida para $u$ e $v$ com o uso da biblioteca PETSc.

A Tabela 5.25 apresenta os erros e as razões de convergência obtidas para as variáveis $u$ e $v$. Os resultados numéricos foram obtidos utilizando o método BCGS (Gradiente Biconjugado estabilizado) e pré-condicionador ILU(0) com tolerância $10^{-12}$.

Observando as razões de convergência obtidas, verificamos que a ordem 2 foi alcançada, tanto na norma $L 2$ quanto na norma infinito. Embora as normas dos erros das variáveis $u$ e $v$ na Tabela 5.25 estão separadas, na biblioteca PETSc, $u$ e $v$ foram resolvidas em um único sistema. Para este sistema, o vetor solução é da forma $[u, v]^{T}$. Quando retornamos as variáveis $u$ e $v$ para o código AMR2D, separamos elas novamente. 

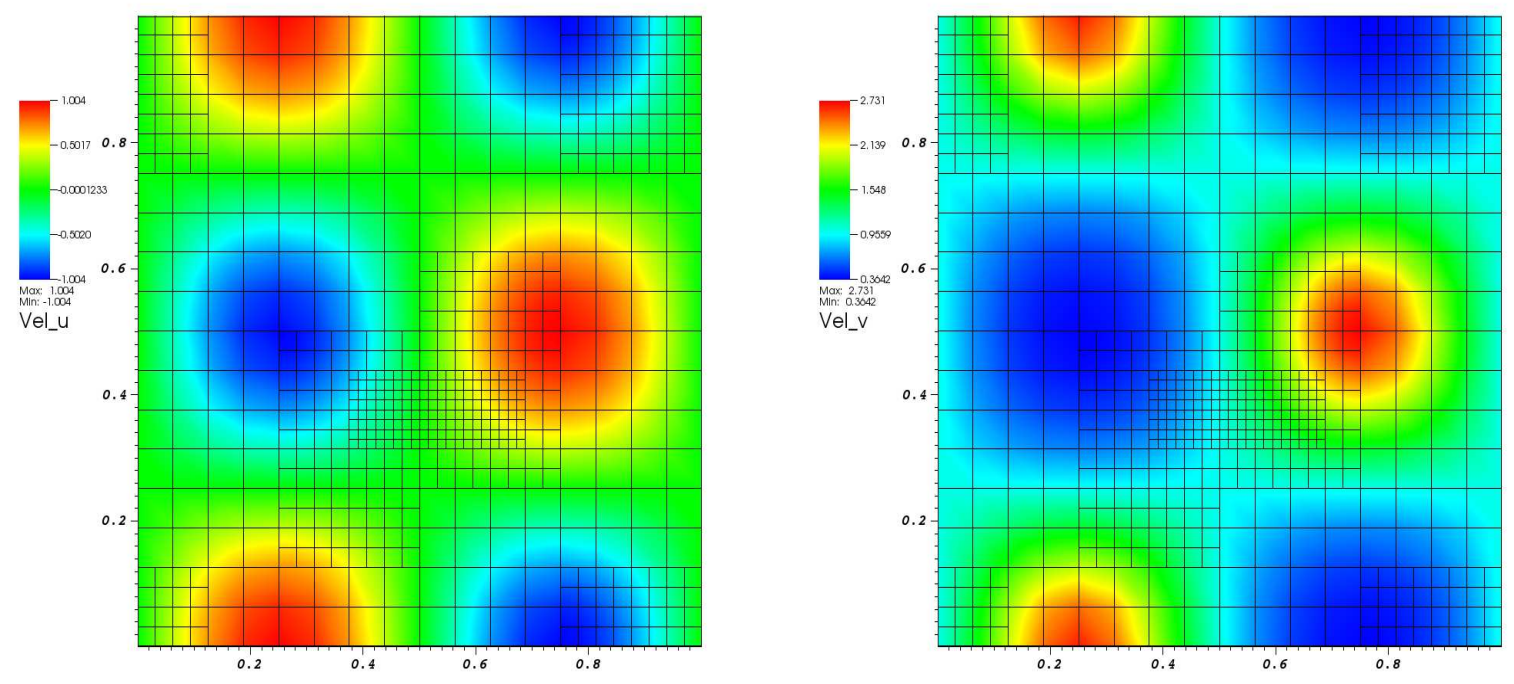

Figura 5.14: Malha composta G4L3 e aproximação de u (à esquerda) e v (à direita), com o operador laplaciano definido nas arestas e condições de contorno de Dirichlet.

\begin{tabular}{ccccccccc}
\hline Malha & $\|e(u)\|_{\infty}$ & $r_{\infty, u}$ & $\|e(u)\|_{2}$ & $r_{2, u}$ & $\|e(v)\|_{\infty}$ & $r_{\infty, v}$ & $\|e(v)\|_{2}$ & $r_{2, v}$ \\
\hline G4L3 & $1.7180 \mathrm{E}-02$ & & $5.6776 \mathrm{E}-03$ & & $3.1158 \mathrm{E}-02$ & & $8.0346 \mathrm{E}-03$ & \\
G5L3 & $4.5835 \mathrm{E}-03$ & 3.7482 & $1.4527 \mathrm{E}-03$ & 3.9084 & $7.7126 \mathrm{E}-03$ & 4.0399 & $1.9826 \mathrm{E}-03$ & 4.0525 \\
G6L3 & $1.1767 \mathrm{E}-03$ & 3.8952 & $3.6801 \mathrm{E}-04$ & 3.9474 & $1.9408 \mathrm{E}-03$ & 3.9740 & $4.9606 \mathrm{E}-04$ & 3.9968 \\
G7L3 & $2.9775 \mathrm{E}-04$ & 3.9519 & $9.2633 \mathrm{E}-05$ & 3.9728 & $4.8905 \mathrm{E}-04$ & 3.9684 & $1.2429 \mathrm{E}-04$ & 3.9913 \\
G8L3 & $7.4872 \mathrm{E}-05$ & 3.9768 & $2.3238 \mathrm{E}-05$ & 3.9863 & $1.2277 \mathrm{E}-04$ & 3.9836 & $3.1119 \mathrm{E}-05$ & 3.9939 \\
\hline
\end{tabular}

Tabela 5.25: Erro e razão de convergência para u e v para a solução da equação de Poisson, com condições de contorno de Dirichlet. 
Este teste nos motivou a utilizar uma representação matricial no Algoritmo 2 (SAMR Método de Uzawa com BiCG-Stab retalho-a-retalho), apresentado na Seção 4.3. O operador discreto $L$, na equação (3.62), representa a matriz correspondente à discretização (5.26).

\subsubsection{Terceiro problema-modelo: equação parabólica para variáveis nas arestas}

Nesta seção, desejamos solucionar uma equação parabólica discretizada em uma malha composta, baseada na estrutura matricial, dada por

$$
\mathbf{u}_{\mathbf{t}}-\nabla \cdot\left[\mu\left(\nabla \mathbf{u}+\nabla \mathbf{u}^{\mathbf{T}}\right)\right]=\mathbf{f}
$$

com condições de contorno do tipo Dirichlet, periódica ou Neumann e condição inicial $\mathbf{u}(x, y, 0)$, onde $\mathbf{u}=[u, v]^{T}, \mathbf{f}=\left[f_{1}, f_{2}\right]^{T}$. As componentes $u$ e $v$ estão definidas no meio das arestas vertical e horizontal de cada célula, respectivamente.

O modelo (5.27) representa um sistema de equações diferenciais parciais para as variáveis $u$ e $v$ dado por

$$
\begin{aligned}
\partial_{t} u & =\mu \nabla^{2} u+\mu \frac{\partial}{\partial x}(\nabla \cdot \mathbf{u})+2 \frac{\partial u}{\partial x} \frac{\partial \mu}{\partial x}+\left(\frac{\partial u}{\partial y}+\frac{\partial v}{\partial x}\right) \frac{\partial \mu}{\partial y}+f_{1} \\
\partial_{t} v & =\mu \nabla^{2} v+\mu \frac{\partial}{\partial y}(\nabla \cdot \mathbf{u})+\left(\frac{\partial u}{\partial y}+\frac{\partial v}{\partial x}\right) \frac{\partial \mu}{\partial x}+2 \frac{\partial v}{\partial y} \frac{\partial \mu}{\partial y}+f_{2} .
\end{aligned}
$$

Tipicamente, equações parabólicas aparecem em nosso trabalho durante a resolução das equações de Navier-Stokes via Método da Projeção. Embora neste trabalho ainda não está sendo usada a discretização que estamos apresentando, futuramente desejamos usá-la com o Método da Projeção matricial segregado e monolítico para estas equações [82].

O terceiro problema modelo foi importante para verificarmos a implementação matricial SAMR, apontando erros na subrotina do cálculo de células fantasmas no código $A M R 2 D$. O teste numérico feito para o terceiro problema modelo também ajudou a verificar a enumeração das arestas, por isso criamos uma malha composta complexa para verificar a ordem de convergência.

Quando $\mu>0$ é constante, o problema (5.27) se reduz à discretização de

$$
\left(\begin{array}{c}
\partial_{t} u \\
\partial_{t} v
\end{array}\right)=\mu\left(\begin{array}{c}
\nabla^{2} u \\
\nabla^{2} v
\end{array}\right)+\left(\begin{array}{l}
f_{1} \\
f_{2}
\end{array}\right),
$$

o qual é um sistema de duas equações desacopladas, que pode ser reescrito na forma

$$
\mathbf{u}_{t}=\mathbf{F}(\mathbf{u})
$$

onde $\mathbf{F}(\mathbf{u})=\mu \nabla^{2} \mathbf{u}+\mathbf{f}$.

Quando $\mu$ for variável, a discretização de (5.28)-(5.29) resulta em um sistema acoplado de equações algébricas. Neste caso, no sistema $(5.30), \mathbf{F}(\mathbf{u})=\nabla \cdot\left[\mu\left(\nabla \mathbf{u}+\nabla \mathbf{u}^{\mathbf{T}}\right)\right]+\mathbf{f}$.

A seguir apresentamos duas discretizações temporais para o sistema (5.28)-(5.29): o Método de Euler Implícito e o Método IMEX. 


\section{Discretização via Método de Euler Implícito}

Inicialmente, solucionamos o sistema de equações (5.30) com o Método de Euler Implícito

$$
\frac{\mathbf{u}^{n+1}-\mathbf{u}^{n}}{\Delta t}=\mathbf{F}^{n+1}
$$

A discretização para cada uma das variáveis $u$ e $v$, quando $\mu$ é constante, é dada por

$$
\begin{aligned}
& \frac{u_{i j}^{n+1}-u_{i, j}^{n}}{\Delta t}=\mu\left(\frac{u_{i+1, j}^{n+1}-2 u_{i j}^{n+1}+u_{i-1, j}^{n+1}}{\Delta x^{2}}+\frac{u_{i, j+1}^{n+1}-2 u_{i j}^{n+1}+u_{i, j-1}^{n+1}}{\Delta y^{2}}\right)+F_{1, i j}^{n+1}, \\
& \frac{v_{i j}^{n+1}-v_{i, j}^{n}}{\Delta t}=\mu\left(\frac{v_{i+1, j}^{n+1}-2 v_{i j}^{n+1}+v_{i-1, j}^{n+1}}{\Delta x^{2}}+\frac{v_{i, j+1}^{n+1}-2 v_{i j}^{n+1}+v_{i, j-1}^{n+1}}{\Delta y^{2}}\right)+F_{2, i j}^{n+1} .
\end{aligned}
$$

Em (5.31)-(5.32), usamos o fato da célula $(i, j)$ ter as arestas oeste e sul indexadas $(i, j)$, a aresta leste indexada $(i+1, j)$ e a aresta norte indexada $(i, j+1)$. Note que as equações (5.31)-(5.32) formam um sistema de equações algébricas lineares.

Dadas as enumerações das arestas correspondentes a cada uma das variáveis $u$ e $v$, criamos a matriz da discretização para (5.31) e para (5.32).

Para $\mu$ variável, a discretização de cada termo de (5.28) é dada por

$$
\begin{aligned}
\left(\partial_{t} u\right)_{i j}^{n+1} & =\frac{u_{i j}^{n+1}-u_{i j}^{n}}{\Delta t} \\
\left(\nabla^{2} u\right)_{i j}^{n+1} & =\frac{u_{i+1 j}^{n+1}-2 u_{i j}^{n+1}+u_{i-1 j}^{n+1}}{\Delta x^{2}}+\frac{u_{i j+1}^{n+1}-2 u_{i j}^{n+1}+u_{i j-1}^{n+1}}{\Delta y^{2}} \\
\left(\frac{\partial u}{\partial x}\right)_{i j}^{n+1} & =\frac{u_{i+1 j}^{n+1}-u_{i-1 j}^{n+1}}{2 \Delta x} \\
\left(\frac{\partial u}{\partial y}\right)_{i j}^{n+1} & =\frac{u_{i j+1}^{n+1}-u_{i j-1}^{n+1}}{2 \Delta y}, \\
\left(\frac{\partial v}{\partial x}\right)_{i j}^{n+1} & =\left(\frac{v_{i j}^{n+1}-v_{i-1 j}^{n+1}}{\Delta x}+\frac{v_{i j+1}^{n+1}-v_{i-1 j+1}^{n+1}}{\Delta x}\right) / 2
\end{aligned}
$$

Note que o termo envolvendo $\nabla \cdot \mathbf{u}$ não é incluido na discretização pois vamos considerar casos em que $\nabla \cdot \mathbf{u}=0$.

Desta forma, a discretização usando o Método de Euler Implícito para a variável $u$ é dada por

$$
\begin{aligned}
\frac{u_{i j}^{n+1}-u_{i j}^{n}}{\Delta t}= & \mu_{i j}\left(\frac{u_{i+1 j}^{n+1}-2 u_{i j}^{n+1}+u_{i-1 j}^{n+1}}{\Delta x^{2}}+\frac{u_{i j+1}^{n+1}-2 u_{i j}^{n+1}+u_{i j-1}^{n+1}}{\Delta y^{2}}\right)+ \\
& +2 \frac{\partial \mu}{\partial x} \frac{u_{i+1 j}^{n+1}-u_{i-1 j}^{n+1}}{2 \Delta x}+\frac{\partial \mu}{\partial y}\left(\frac{u_{i j+1}^{n+1}-u_{i j-1}^{n+1}}{2 \Delta y}+\right. \\
& \left.+\frac{v_{i j}^{n+1}-v_{i-1 j}^{n+1}+v_{i j+1}^{n+1}-v_{i-1 j+1}^{n+1}}{2 \Delta x}\right)+f_{1, i j}^{n+1} .
\end{aligned}
$$


Agrupando os termos de mesmo índice, escrevemos a forma matricial como

$$
\begin{aligned}
& \left(-\frac{\mu_{i j}}{\Delta x^{2}}+\frac{\partial \mu}{\partial x} \frac{1}{\Delta x}\right) u_{i-1 j}^{n+1}+\left(-\frac{\mu_{i j}}{\Delta y^{2}}+\frac{\partial \mu}{\partial y} \frac{1}{2 \Delta y}\right) u_{i j-1}^{n+1}+ \\
& +\left(\frac{1}{\Delta t}+2 \mu_{i j}\left(\frac{1}{\Delta x^{2}}+\frac{1}{\Delta y^{2}}\right)\right) u_{i j}^{n+1}+\left(-\frac{\mu_{i j}}{\Delta y^{2}}-\frac{\partial \mu}{\partial y} \frac{1}{2 \Delta y}\right) u_{i j+1}^{n+1}+ \\
& +\left(-\frac{\mu_{i j}}{\Delta x^{2}}-\frac{\partial \mu}{\partial x} \frac{1}{\Delta x}\right) u_{i+1 j}^{n+1}+\frac{\partial \mu}{\partial y} \frac{1}{2 \Delta x} v_{i-1 j}^{n+1}+\frac{\partial \mu}{\partial y} \frac{1}{2 \Delta x} v_{i-1 j+1}^{n+1}+ \\
& -\frac{\partial \mu}{\partial y} \frac{1}{2 \Delta x} v_{i j}^{n+1}-\frac{\partial \mu}{\partial y} \frac{1}{2 \Delta x} v_{i j+1}^{n+1}=f_{1, i j}^{n+1}+\frac{1}{\Delta t} u_{i j}^{n} .
\end{aligned}
$$

De forma análoga, pode ser obtida a forma matricial para (5.29) empregando o Método de Euler Implícito.

\section{Discretização via métodos implícitos-explícitos (IMEX)}

Na Seção 3.2.2, descrevemos os métodos IMEX aplicados às equações de Navier-Stokes. Nesta seção, vamos aproximar (5.27) empregando a metodologia IMEX (3.14), onde $\mathbf{g}=\nabla \cdot\left[\mu\left(\nabla \mathbf{u}+\nabla \mathbf{u}^{\mathbf{T}}\right)\right]$, $\mathbf{f}=\mathbf{f}^{\text {man }}, \quad \mathbf{f}^{\text {man }}$ é o termo forçante manufaturado e $\alpha_{i}, \theta_{i}$ e $\beta_{i}$ são dados pelas equações (3.16)(3.18).

Assim, a representação matricial para (5.28) é obtida a partir de

$$
\begin{aligned}
& u_{i-1 j}^{n+1}\left(\frac{\theta_{2}}{\Delta x} \frac{\partial \mu_{i j}^{n+1}}{\partial x}-\frac{\theta_{2}}{\Delta x^{2}} \mu_{i j}^{n+1}\right)+u_{i j-1}^{n+1}\left(\frac{\theta_{2}}{2 \Delta y} \frac{\partial \mu_{i j}^{n+1}}{\partial y}-\frac{\theta_{2}}{\Delta y^{2}} \mu_{i j}^{n+1}\right)+ \\
+ & u_{i j}^{n+1}\left(\frac{\alpha_{2}}{\Delta t^{n+1}}+\frac{2 \theta_{2}}{\Delta x^{2}} \mu_{i j}^{n+1}+\frac{2 \theta_{2}}{\Delta y^{2}} \mu_{i j}^{n+1}\right)+u_{i j+1}^{n+1}\left(-\frac{\theta_{2}}{2 \Delta y} \frac{\partial \mu_{i j}^{n+1}}{\partial y}-\frac{\theta_{2}}{\Delta y^{2}} \mu_{i j}^{n+1}\right)+ \\
+ & u_{i+1 j}^{n+1}\left(-\frac{\theta_{2}}{\Delta x} \frac{\partial \mu_{i j}^{n+1}}{\partial x}-\frac{\theta_{2}}{\Delta x^{2}} \mu_{i j}^{n+1}\right)+v_{i-1 j}^{n+1}\left(\frac{\theta_{2}}{2 \Delta x} \frac{\partial \mu_{i j}^{n+1}}{\partial y}\right)+ \\
+ & v_{i-1 j+1}^{n+1}\left(\frac{\theta_{2}}{2 \Delta x} \frac{\partial \mu_{i j}^{n+1}}{\partial y}\right)+v_{i j}^{n+1}\left(-\frac{\theta_{2}}{2 \Delta x} \frac{\partial \mu_{i j}^{n+1}}{\partial y}\right)+v_{i j+1}^{n+1}\left(-\frac{\theta_{2}}{2 \Delta x} \frac{\partial \mu_{i j}^{n+1}}{\partial y}\right) \\
= & \theta_{1} g_{1}\left(u^{n}, v^{n}\right)_{i j}+\theta_{0} g_{1}\left(u^{n-1}, v^{n-1}\right)_{i j}+\beta_{1} f_{1}\left(u^{n}, v^{n}\right)_{i j}+\beta_{0} f_{1}\left(u^{n-1}, v^{n-1}\right)_{i j}+ \\
& -\frac{\alpha_{1} u_{i j}^{n}+\alpha_{2} u_{i j}^{n-1}}{\Delta t^{n+1}} .
\end{aligned}
$$

De forma análoga, pode ser obtida a forma matricial para (5.29) com a metodologia IMEX.

\section{Verificações numéricas}

As verificações numéricas para esse problema modelo foram feitas com o uso da biblioteca PETSc em um único processo. Para resolver o sistema linear usamos o método GMRES com o précondicionador ILU(0). A tolerância utilizada na solução do sistema foi $10^{-12}$, com número máximo de iterações 10000. Na biblioteca PETSc não fixamos o tipo de matriz usada para armazenar a discretização. Assim a biblioteca PETSc decide qual tipo de matriz usar.

Neste teste, a matriz está mudando a cada passo de tempo, embora estejamos usando um tamanho de passo $\Delta t$ constante. O tempo final escolhido foi $t_{f}=0.5$, e $\Delta t=\min \left(\Delta x_{l t o p}, \Delta y_{l t o p}\right)$ 
depende do espaçamento da malha do nível mais fino. As funções usadas neste teste foram

$$
\begin{aligned}
u(x, y, t) & =-\partial_{y} \varphi(x, y, t), \\
v(x, y, t) & =\partial_{x} \varphi(x, y, t), \\
\mu(x) & =(1+h)+h \tanh [2 \pi(x-0.5)],
\end{aligned}
$$

onde $\varphi(x, y, t)=\sin (\pi x)^{2} \sin (\pi y)^{2} \cos (\pi t) / \pi, \nabla \cdot \mathbf{u}=0$ e $h$ controla a variação de $\mu$, neste caso $h=100$.

A Figura 5.15 mostra a malha composta utilizada. Consideramos esta malha suficientemente complexa e aleatória para verificar as enumerações das arestas. A enumeração nas arestas foi complicada e foram necessários testes em diferentes tipos de malhas compostas. Na Figura 5.15, a malha à esquerda possui $8 \times 8$ células na malha base e mais dois níveis de refinamento. A malha à direita possui $16 \times 16$ células e mais dois níveis de refinamento.
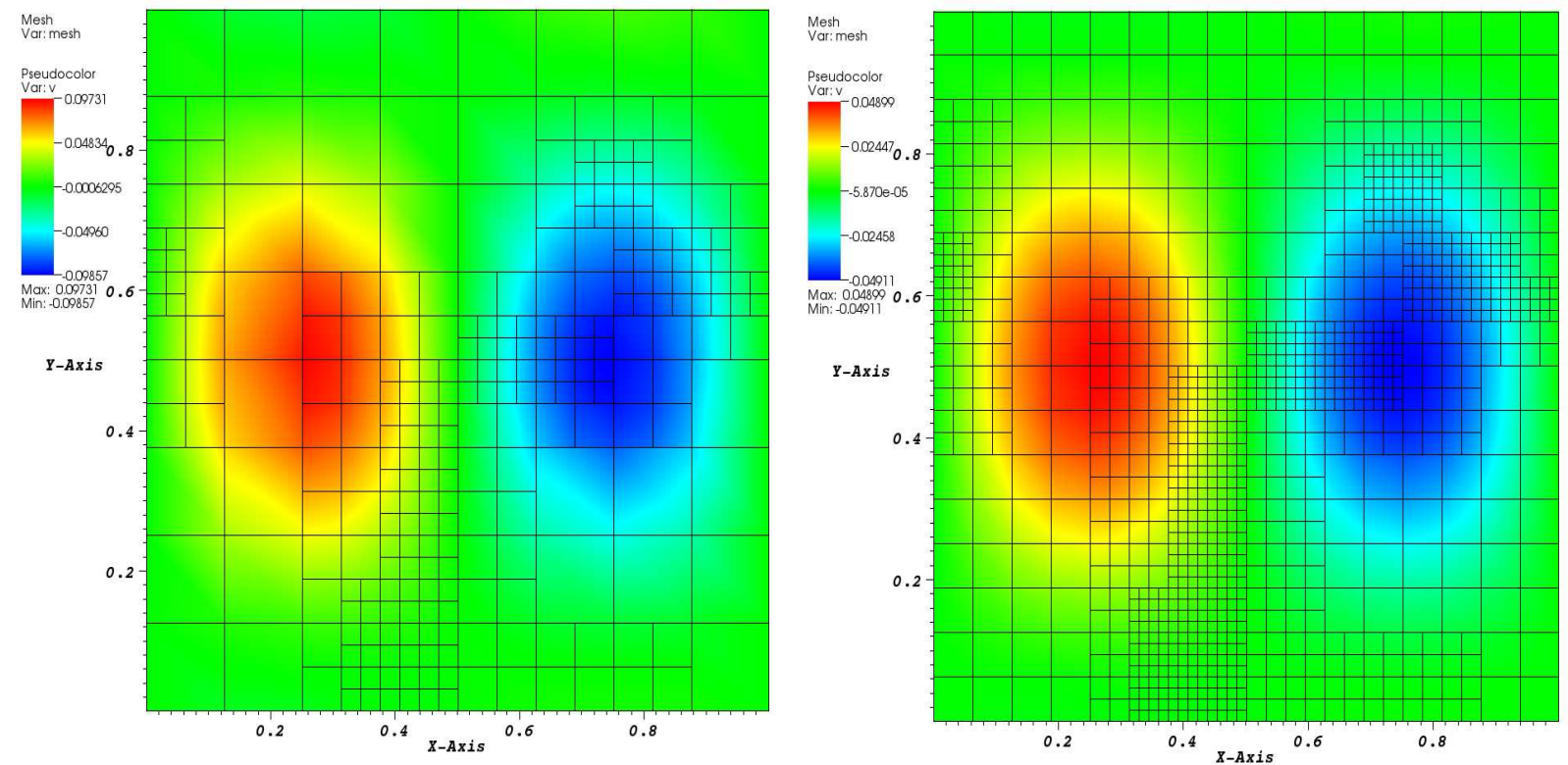

Figura 5.15: Malha composta (à esquerda) e malha composta refinada (à direita) com a aproximação de $v$.

Note que os retalhos da malha composta estão fixos e isto deve ser satisfeito durante a verificação numérica. Antes não podíamos fazer verificações numéricas com dois ou mais níveis de refinamento (além da malha base), pois não conseguíamos garantir que os retalhos permanecessem fixos quando a malha era refinada. Durante a realização deste trabalho, nós incluimos estes tipos de testes com malhas compostas e a ideia pode ser estendida para o código AMR3D.

A Figura 5.16 mostra o padrão de esparsidade da matriz de discretização usando o Método de Euler Implícito. Note que a matriz apresenta um padrão com diagonais inferiores de elementos não nulos. Isto ocorre porque $\mu$ foi escolhida como função que depende apenas de $x$. Desta forma, os termos cruzados que têm um produto com $\frac{\partial \mu}{\partial y}=0$ não aparecem.

A Tabela 5.26 apresenta os erros na norma $L 2$, a razão de convergência para $u$ e $v$ e o número de iterações do método que resolve o sistema no último passo de tempo. Vale lembrar que o Método de 


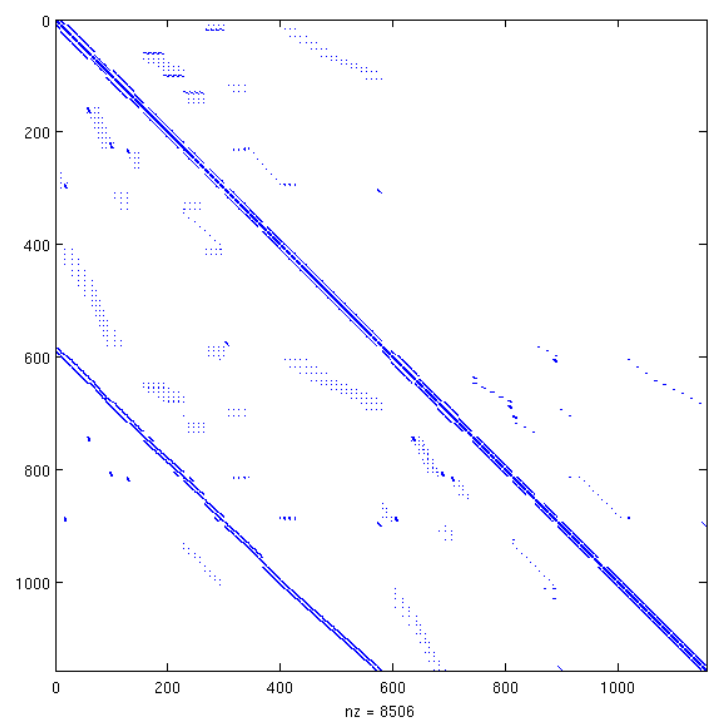

Figura 5.16: Padrão de esparsidade da matriz na equação de difusão com o método de Euler Implícito.

\begin{tabular}{cccccc}
\hline Malha & $\|e(u)\|_{2}$ & $r_{2, u}$ & $\|e(v)\|_{2}$ & $r_{2, v}$ & IterS \\
\hline $8 \times 8 L 3$ & $4.266 \mathrm{e}-04$ & & $3.903 \mathrm{e}-04$ & & 49 \\
$16 \times 16 L 3$ & $7.662 \mathrm{e}-05$ & 5.567 & $8.747 \mathrm{e}-05$ & 4.462 & 128 \\
$32 \times 32 L 3$ & $1.683 \mathrm{e}-05$ & 4.552 & $2.059 \mathrm{e}-05$ & 4.248 & 260 \\
$64 \times 64 L 3$ & $4.116 \mathrm{e}-06$ & 4.089 & $4.728 \mathrm{e}-06$ & 4.354 & 565 \\
$128 \times 128 L 3$ & $1.038 \mathrm{e}-06$ & 3.953 & $9.733 \mathrm{e}-07$ & 4.857 & 1521 \\
\hline
\end{tabular}

Tabela 5.26: Erros, ordem de convergência e número de iterações na equação de difusão com o método de Euler Implícito. 
Euler Implícito possui ordem de convergência igual a 1. Entretanto, os valores obtidos na Tabela 5.26 sugerem que obtivemos ordem 2. Outros exemplos de funções testes devem ser utilizados para verificar se não foi alguma simetria do problema que gerou o aumento da ordem de convergência. A coluna IterS na Tabela 5.26 é o número de iterações na última solução matricial dada pela biblioteca PETSc. Lembrando que a cada passo de tempo um sistema é resolvido.

No caso da discretização com a metodologia IMEX, usamos os coeficientes do Método de Gear com passo de tempo constante obtidos $\operatorname{com} \gamma=1$ e $c=0$, ou seja

$$
\alpha_{2}=3 / 2, \alpha_{1}=-2, \alpha_{0}=1 / 2, \theta_{2}=1, \theta_{1}=\theta_{0}=0, \beta_{1}=2 \text { e } \beta_{0}=-1 .
$$

A Tabela 5.27 apresenta os erros na norma $L 2$, a razão de convergência para $u$ e $v$ e o número de iterações do método que resolve o sistema no último passo de tempo.

\begin{tabular}{cccccc}
\hline Malha & $\|e(u)\|_{2}$ & $r_{2, u}$ & $\|e(v)\|_{2}$ & $r_{2, v}$ & IterS \\
\hline $8 \times 8 L 3$ & $4.266 \mathrm{e}-04$ & & $3.903 \mathrm{e}-04$ & & 158 \\
$16 \times 16 L 3$ & $4.0613 \mathrm{e}-05$ & 4.567 & $3.0517 \mathrm{e}-05$ & 5.112 & 403 \\
$32 \times 32 L 3$ & $8.581 \mathrm{e}-06$ & 4.733 & $5.992 \mathrm{e}-06$ & 5.092 & 1092 \\
$64 \times 64 L 3$ & $1.856 \mathrm{e}-06$ & 4.622 & $1.247 \mathrm{e}-06$ & 4.806 & 3627 \\
\hline
\end{tabular}

Tabela 5.27: Erro, ordem de convergência e número de iterações na equação de difusão com o Método de Gear.

Neste caso, obtemos a ordem de convergência esperada igual a 2. A ordem de convergência é verificada na Tabela 5.27 ao observarmos as reazões de convergência. Comparando a coluna IterS nas tabelas 5.26 e 5.27, observamos o aumento do número de iterações do Método GMRES da biblioteca PETSc quando utilizamos o Método de Gear.

Os problemas-modelo mostrados nesta seção são o início e a motivação do estudo da solução matricial na forma segregada das equações de Navier-Stokes no SAMR [82].

\subsection{Verificação do tensor viscoelástico}

Nesta Seção, apresentamos a solução numérica para um tensor viscoelástico dado pelos modelos Oldroyd-B e FENE-P, os quais têm o termo advectivo discretizado com um esquema ENO de terceira ordem. A discretização temporal é feita com um método de Runge-Kutta de segunda ordem (RK2) com propriedade TVD (total variation diminishing).

\subsubsection{Verificação do termo de advecção com ENO3}

Na Seção 3.4.1 do Capítulo 3, apresentamos a discretização para o termo advectivo dos modelos Oldroyd-B e FENE-P do tensor viscoelástico. Fazemos dois testes diferentes: um onde a aproximação é para variáveis suaves e outro onde há um salto.

A aproximação do termo advectivo empregando um esquema ENO de terceira ordem (ENO3) gerou particular dificuldade dado que ela precisa de uma camada de três células fantasmas ao redor de cada retalho. Inicialmente, ao empregarmos malhas compostas não obtivemos os resultados esperados. O motivo disto foi investigado e descobrimos alguns problemas de implementação que passaram desapercebidos até então pois o código AMR2D havia sido usado supondo apenas uma camada de células fantasmas. Embora o diagnóstico do problema tenha sido relativamente fácil, foi 
apenas com grande esforço que conseguimos gerar exemplos que expuseram com clareza os pontos que demandavam correção. Nos testes reportados a seguir, tivemos o cuidado de procurar usar malhas sem nenhuma particularidade ou simetria aparente. Reutilizamos a mesma malha composta empregada quando estudamos a enumeração de faces na formação de matrizes (Figura 5.15).

Consideramos $p, u$ e $v$ funções suaves dadas por

$$
\begin{aligned}
& u=e^{\sin (2 \pi x) \cos (2 \pi y)}, \\
& v=e^{\sin (2 \pi x) \cos (2 \pi y)}, \\
& p=t \sin (2 \pi x) \cos (2 \pi y),
\end{aligned}
$$

e condição de contorno de Dirichlet. Mostramos na Tabela 5.28 o erro e a ordem de convergência na discretização para uma variável $\phi$ dada por

$$
\phi=\mathbf{u} \cdot \nabla p=u \frac{\partial p}{\partial x}+v \frac{\partial p}{\partial y}
$$

empregando o esquema ENO3. Ainda nos testes executados e reportados a seguir, não consideramos a aproximação temporal dada pelo RK2. Empregamos apenas a discretização espacial.

Com os valores obtidos na Tabela 5.28, podemos ver que a ordem de convergência na norma L2 tende a quatro. Esperávamos que a aproximação fosse, claro, de ordem três e não encontramos explicação aparente para tal comportamento. Por outro lado, na norma infinito obtivemos que a ordem de convergência tende a dois, neste caso isto pode ser dado pelo grau de aproximação das células fantasmas na fronteira com condição de contorno de Dirichlet que é de segunda ordem. Os resultados obtidos nesse exemplo serão estudados mais profundamente futuramente. A aproximação do termo advectivo, encontramos no Capítulo 3.

\begin{tabular}{ccccc}
\hline Malha & $\|e(\phi)\|_{\infty}$ & $r_{\infty}$ & $\|e(\phi)\|_{2}$ & $r_{2}$ \\
\hline$G 5 L 1$ & $8.2601 \mathrm{E}-01$ & & $3.3693 \mathrm{E}-02$ & \\
$G 6 L 1$ & $2.0597 \mathrm{E}-01$ & 4.010 & $2.0156 \mathrm{E}-03$ & 16.716 \\
$G 7 L 1$ & $5.1455 \mathrm{E}-02$ & 4.003 & $1.2405 \mathrm{E}-04$ & 16.249 \\
$G 8 L 1$ & $1.2861 \mathrm{E}-02$ & 4.001 & $7.7227 \mathrm{E}-06$ & 16.063 \\
G9L1 & $3.2152 \mathrm{E}-03$ & 4.000 & $4.8220 \mathrm{E}-07$ & 16.015 \\
\hline G5L3 & $8.2601 \mathrm{E}-01$ & & $1.5805 \mathrm{E}-02$ & \\
G6L3 & $2.0597 \mathrm{E}-01$ & 4.010 & $8.9563 \mathrm{E}-04$ & 17.647 \\
G7L3 & $5.1455 \mathrm{E}-02$ & 4.003 & $5.4022 \mathrm{E}-05$ & 16.579 \\
G8L3 & $1.2861 \mathrm{E}-02$ & 4.001 & $3.3304 \mathrm{E}-06$ & 16.221 \\
G9L3 & $3.2152 \mathrm{E}-03$ & 4.000 & $2.0696 \mathrm{E}-07$ & 16.092 \\
\hline
\end{tabular}

Tabela 5.28: Erro e ordem de convergência usando ENOЗ com condição de Dirichlet para malhas uniforme e composta.

Realizamos o mesmo teste em malha composta mas, desta vez, com condição de contorno mista: temos condição periódica na direção $x$ e de Dirichlet em $y$. Obtivemos os resultados que estão na Tabela 5.29 e, novamente, a ordem de convergência tende a quatro na norma $L 2$ e a dois na norma infinito. 


\begin{tabular}{ccccc}
\hline Malha & $\|e(\phi)\|_{\infty}$ & $r_{\infty}$ & $\|e(\phi)\|_{2}$ & $r_{2}$ \\
\hline$G 5 L 3$ & $8.1081 \mathrm{E}-01$ & & $1.2037 \mathrm{E}-02$ & \\
$G 6 L 3$ & $2.0500 \mathrm{E}-01$ & 3.955 & $6.8262 \mathrm{E}-04$ & 17.634 \\
$G 7 L 3$ & $5.1394 \mathrm{E}-02$ & 3.989 & $4.1316 \mathrm{E}-05$ & 16.522 \\
$G 8 L 3$ & $1.2858 \mathrm{E}-02$ & 3.997 & $2.5523 \mathrm{E}-06$ & 16.188 \\
G9L3 & $3.2150 \mathrm{E}-03$ & 3.999 & $1.5882 \mathrm{E}-07$ & 16.070 \\
\hline
\end{tabular}

Tabela 5.29: Erro e ordem de convergência usando ENO3 com condição mista de Dirichlet e periódica. Malha composta.

A seguir, apresentamos um teste que simula uma descontinuidade (salto) empregando as funções

$$
\begin{aligned}
& u=(1+h)+h * \tanh \left(2 \pi d\left(x-x_{c}\right)\right), \\
& v=(1+h)+h * \tanh \left(2 \pi d\left(x-x_{c}\right)\right), \\
& p=(1+h)+h * \tanh \left(2 \pi d\left(x-x_{c}\right)\right),
\end{aligned}
$$

onde $x_{c}=0.5, d=4$ e $h$ controla a variação de $u, v$ e $p$, dado neste caso por $h=10$.

Os resultados obtidos neste caso, usando condição de contorno mista como no teste anterior, são mostrados na Tabela 5.30. Neste caso, usamos uma malha uniforme e uma malha composta com três níveis de refinamento para a qual a região de refinamento se encontra onde temos o salto, como vemos na Figura 5.17. Nesta figura, temos a malha composta recobrindo a região do domínio com variação e também representamos os valores da aproximação obtida para $\phi$.
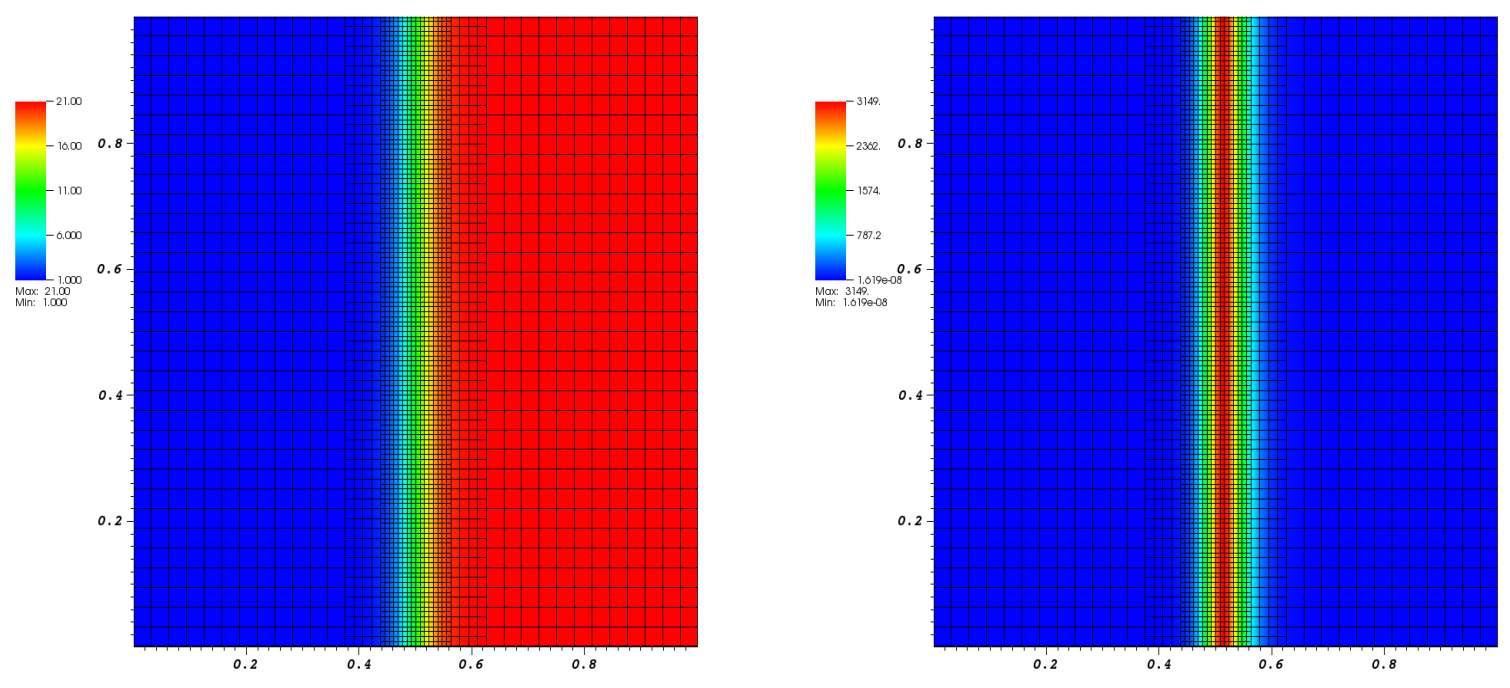

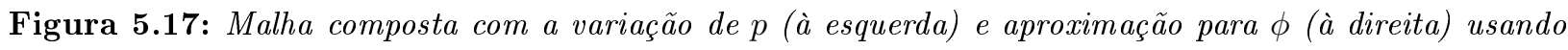
ENO3 com condições de contorno mistas de Dirichlet e periódica.

Dos resultados mostrados na Tabela 5.30, percebemos que, como era de se esperar, os valores dos erros na malha composta são menores que os da malha uniforme. 


\begin{tabular}{ccccc}
\hline Malha & $\|e(p)\|_{\infty}$ & $r_{\infty}$ & $\|e(p)\|_{2}$ & $r_{2}$ \\
\hline$G 5 L 1$ & $8.6325 \mathrm{E}+02$ & & $2.5822 \mathrm{E}+04$ & \\
$G 6 L 1$ & $7.1974 \mathrm{E}+01$ & 11.994 & $1.4181 \mathrm{E}+02$ & 182.087 \\
$G 7 L 1$ & $8.7154 \mathrm{E}+00$ & 8.258 & $1.1868 \mathrm{E}+00$ & 119.484 \\
$G 8 L 1$ & $1.2786 \mathrm{E}+00$ & 6.816 & $5.2929 \mathrm{E}-02$ & 22.423 \\
$G 9 L 1$ & $3.7216 \mathrm{E}-01$ & 3.436 & $5.6644 \mathrm{E}-03$ & 9.344 \\
$G 10 L 1$ & $1.0044 \mathrm{E}-01$ & 3.705 & $4.5761 \mathrm{E}-04$ & 12.378 \\
\hline$G 5 L 3$ & $1.1017 \mathrm{E}+01$ & & $4.4977 \mathrm{E}+00$ & \\
$G 6 L 3$ & $3.5815 \mathrm{E}+00$ & 3.076 & $3.5694 \mathrm{E}-01$ & 12.600 \\
$G 7 L 3$ & $1.0638 \mathrm{E}+00$ & 3.367 & $2.5352 \mathrm{E}-02$ & 14.079 \\
$G 8 L 3$ & $1.4947 \mathrm{E}-01$ & 7.117 & $9.3696 \mathrm{E}-04$ & 27.058 \\
$G 9 L 3$ & $2.6079 \mathrm{E}-02$ & 5.732 & $3.9231 \mathrm{E}-05$ & 23.883 \\
\hline
\end{tabular}

Tabela 5.30: Erro e ordem de convergência em malha uniforme e em malha composta empregando ENO3 com condição de contorno mista de Dirichlet e periódica.

\subsubsection{Verificação do Modelo Oldroyd-B}

Para o teste de verificação da ordem de convergência da discretização empregada para o Modelo Oldroyd-B, usamos as forças $f_{x x}^{\text {man }}, f_{x y}^{\text {man }}$ e $f_{y y}^{\text {man }}$ para a solução manufaturada dadas por

$$
\begin{aligned}
& f_{x x}^{\text {man }}=\frac{\partial S_{x x}}{\partial t}+\left(u \frac{\partial S_{x x}}{\partial x}+v \frac{\partial S_{x x}}{\partial y}\right)-2\left(S_{x x} \frac{\partial u}{\partial x}+S_{x y} \frac{\partial u}{\partial y}\right)+W_{i}^{-1}\left(S_{x x}-1\right), \\
& f_{x y}^{\text {man }}=\frac{\partial S_{x y}}{\partial t}+\left(u \frac{\partial S_{x y}}{\partial x}+v \frac{\partial S_{x y}}{\partial y}\right)-\left(\frac{\partial v}{\partial x} S_{x x}+\frac{\partial u}{\partial y} S_{y y}\right)+W_{i}^{-1} S_{x y}, \\
& f_{y y}^{\text {man }}=\frac{\partial S_{y y}}{\partial t}+\left(u \frac{\partial S_{y y}}{\partial x}+v \frac{\partial S_{x x}}{\partial y}\right)-2\left(\frac{\partial v}{\partial x} S_{x y}+\frac{\partial v}{\partial y} S_{y y}\right)+W_{i}^{-1}\left(S_{y y}-1\right) .
\end{aligned}
$$

No RK2, empregamos para o teste manufaturado, por exemplo na componente $S_{x x}$ do tensor

$$
\frac{\partial S_{x x}}{\partial t}=g_{x x}\left(t, u, v, S_{x x}, S_{x y}\right)
$$

a função $g_{x x}(2.30)$ definida no Capítulo 2. Aqui, esta função é dada por

$$
g_{x x}=-\left(u \frac{\partial S_{x x}}{\partial x}+v \frac{\partial S_{x x}}{\partial y}\right)+2\left(S_{x x} \frac{\partial u}{\partial x}+S_{x y} \frac{\partial u}{\partial y}\right)-W_{i}^{-1}\left(S_{x x}-1\right)+f_{x x}^{\operatorname{man}} .
$$

Da mesma forma, definimos as funções $g_{x y}$ e $g_{y y}$.

As funções usadas para os testes usando condição de contorno de Dirichlet, foram

$$
\begin{aligned}
& S_{x x}(x, y ; t)=-2 \cos (\pi t) \sin ^{2}(\pi x) \cos (\pi y) \sin (\pi y), \\
& S_{x y}(x, y ; t)=t \sin (2 \pi x) \sin (2 \pi y), \\
& S_{y y}(x, y ; t)=e^{-t} \sin (2 \pi x) \sin (2 \pi y), \\
& u(x, y ; t)=-2 \cos (\pi t) \sin ^{2}(\pi x) \cos (\pi y) \sin (\pi y), \\
& v(x, y ; t)=2 \cos (\pi t) \cos (\pi x) \sin (\pi x) \sin ^{2}(\pi y), \\
& \nabla \cdot \mathbf{u}=0, \quad t_{f}=2.5 \quad \Omega=[0,1] \times[0,1] .
\end{aligned}
$$

Lembrando que quando apresentamos o modelo matemático para o tensor simplificamos o termo 
dado pela incompressibilidade do fluido. Devido a isto, é necessário escolher $\mathbf{u}$ tal que $\nabla \cdot \mathbf{u}=0$. A discretização usada foi a definida no Capítulo 3.

Na Tabela 5.31, estão os erros obtidos para cada uma das componentes tensoriais de $\mathbf{S}$ em malha uniforme e em malha composta contendo três níveis de refinamento, dada na Figura 5.1. Incluímos a quantidade de passos no tempo nstep tomados para chegar ao tempo final, lembrando que $\Delta t$ deve satisfazer $\Delta t \leq \frac{1}{\Delta t_{u}+\Delta t_{v}}$, onde $\Delta t_{u}=\frac{|u \max |}{\min (\Delta x, \Delta y)_{l}}$ e $\Delta t_{v}=\frac{|v \max |}{\min (\Delta x, \Delta y)_{l}}$. Mais uma vez, esperávamos segunda ordem de convergência, porém obtivemos para cada uma das componentes um comportamento que tende para ordem quatro (comportamento superconvergente).

\begin{tabular}{cccccccc}
\hline Malha & nstep & $\|e(S x x)\|_{2}$ & $r_{2} S_{x x}$ & $\|e(S x y)\|_{2}$ & $r_{2} S_{x y}$ & $\| e($ Syy $) \|_{2}$ & $r_{2} S_{y y}$ \\
\hline G5L1 & 29 & $1.1433 \mathrm{E}-06$ & & $1.6496 \mathrm{E}-06$ & & $3.5895 \mathrm{E}-06$ & \\
G6L1 & 58 & $7.2984 \mathrm{E}-08$ & 15.6643 & $9.8889 \mathrm{E}-08$ & 16.6818 & $2.1049 \mathrm{E}-07$ & 17.0533 \\
G7L1 & 116 & $4.6012 \mathrm{E}-09$ & 15.8620 & $6.0463 \mathrm{E}-09$ & 16.3552 & $1.2767 \mathrm{E}-08$ & 16.4863 \\
G8L1 & 231 & $2.8891 \mathrm{E}-10$ & 15.9259 & $3.7368 \mathrm{E}-10$ & 16.1804 & $7.8716 \mathrm{E}-10$ & 16.2196 \\
\hline G5L3 & 116 & $8.5270 \mathrm{E}-07$ & & $1.1556 \mathrm{E}-06$ & & $2.1200 \mathrm{E}-06$ & \\
G6L3 & 231 & $5.4153 \mathrm{E}-08$ & 15.7459 & $7.0159 \mathrm{E}-08$ & 16.4712 & $1.2066 \mathrm{E}-07$ & 17.5704 \\
G7L3 & 462 & $3.4303 \mathrm{E}-09$ & 15.7869 & $4.3202 \mathrm{E}-09$ & 16.2396 & $7.2357 \mathrm{E}-09$ & 16.6755 \\
G8L3 & 923 & $2.1721 \mathrm{E}-10$ & 15.7927 & $2.6841 \mathrm{E}-10$ & 16.0958 & $4.4161 \mathrm{E}-10$ & 16.3848 \\
\hline
\end{tabular}

Tabela 5.31: Erro e ordem de convergência nas malhas uniforme (L1) e composta (L3) para cada uma das componentes do tensor $\mathbf{S}$ com o Modelo Oldroyd-B.

\subsubsection{Verificação do Modelo FENE-P}

Para verificamos a correta implementação do Modelo FENE-P, empregamos as funções

$$
\begin{aligned}
S x x & =e^{-t} \sin (2 \pi x) \sin (2 \pi y) \\
S x y & =t \sin (2 \pi x) \sin (2 \pi y) \\
S y y & =e^{-t} \sin (2 \pi x) \sin (2 \pi y), \\
u(x, y ; t) & =-2 \cos (\pi t) \sin ^{2}(\pi x) \cos (\pi y) \sin (\pi y), \\
v(x, y ; t) & =2 \cos (\pi t) \cos (\pi x) \sin (\pi x) \sin ^{2}(\pi y), \\
\nabla \cdot \mathbf{u} & =0 .
\end{aligned}
$$

Os parâmetros adotados foram $t \in[0,0.25], W_{i}=10$ e $l=7$, e condições de contorno de Dirichlet puras.

O Modelo FENE-P é dado pelas equações (2.31)-(2.32), onde, para o teste manufaturado, precisamos definir o termo forçante do lado direito que depende da solução exata $\mathbf{f}_{m a n}$. Dessa forma, no teste manufaturado para o Modelo FENE-P usamos

$$
\begin{gathered}
\partial_{t} \mathbf{C}+\mathbf{u} . \nabla \mathbf{C}-\left(\nabla \mathbf{u} \mathbf{C}+\mathbf{C} \nabla \mathbf{u}^{T}\right)+\frac{1}{W_{i}}(\mathbf{S}-I)=\mathbf{f}_{m a n}, \\
\mathbf{S}=\frac{\mathbf{C}}{1-\left(\frac{\operatorname{tr} \mathbf{C}}{l^{2}}\right)}
\end{gathered}
$$


onde, por exemplo, para a componente $S_{x x}$ temos

$$
\begin{gathered}
\frac{\partial C_{x x}}{\partial t}+\left(u \frac{\partial C_{x x}}{\partial x}+v \frac{\partial C_{x x}}{\partial y}\right)-2\left(\frac{\partial u}{\partial x} C_{x x}+\frac{\partial u}{\partial y} C_{x y}\right)+\omega e^{-1}\left(S_{x x}-1\right)=f_{x x}^{\operatorname{man}} \\
S_{x x}=\frac{1}{1-\left(\operatorname{tr}(\mathbf{C}) / l^{2}\right)} C_{x x} .
\end{gathered}
$$

Nota-se que nas funções empregadas para o teste em (5.35), nenhuma tem um valor específico para o tensor C. Então, para conseguir definir $f_{x x}$, devemos usar a relação que define o tensor $\mathbf{C}$ em função do tensor dado $\mathbf{S}_{\mathbf{p}}$, seguindo a equação (2.36). Por intermédio desta equação também definimos a condição inicial para $\mathbf{C}$.

A função $h$ definida no Capítulo 2 em (2.39), para esta mesma componente do tensor é

$$
h_{x x}=-\left(u \frac{\partial C_{x x}}{\partial x}+v \frac{\partial C_{x x}}{\partial y}\right)+2\left(C_{x x} \frac{\partial u}{\partial x}+C_{x y} \frac{\partial u}{\partial y}\right)-W_{i}^{-1}\left(S_{x x}-1\right)+f_{x x}^{\operatorname{man}} .
$$

De forma análoga, definimos as funções $g_{x y}$ e $g_{y y}$.

Para a malha composta dada na Figura 5.1, com nível base $16 \times 16$ e três níveis de refinamento, obtemos os valores mostrados na Tabela 5.32. Neste caso, não obtivemos a ordem de convergência esperada, segunda ordem. Todas as componentes do tensor apresentam a mesma ordem de convergência, ficando mais claro o comportamento para as componentes $S x x$ e $S x y$, no caso da solução manufaturada empregada. Por outro lado, a componente Syy, que depende da componente Sxy, não tem um comportamento natural na forma de decaimento do erro, como podemos ver na última coluna da Tabela 5.32, mas ele diminui, exibindo um comportamento que tende à quarta ordem.

\begin{tabular}{ccccccc}
\hline Malha & $\|e(S x x)\|_{2}$ & $r_{2} S_{x x}$ & $\|e(S x y)\|_{2}$ & $r_{2} S_{x y}$ & $\|e(S y y)\|_{2}$ & $r_{2} S_{y y}$ \\
\hline G4L3 & $7.7854 \mathrm{E}-05$ & & $1.8290 \mathrm{E}-05$ & & $2.8429 \mathrm{E}-04$ & \\
G5L3 & $1.8491 \mathrm{E}-06$ & 42.1030 & $1.2645 \mathrm{E}-06$ & 14.4646 & $1.5327 \mathrm{E}-05$ & 18.5487 \\
G6L3 & $1.1332 \mathrm{E}-07$ & 16.3182 & $8.2581 \mathrm{E}-08$ & 15.3122 & $3.5324 \mathrm{E}-07$ & 43.3889 \\
G7L3 & $7.4602 \mathrm{E}-09$ & 15.1896 & $5.3249 \mathrm{E}-09$ & 15.5084 & $1.8188 \mathrm{E}-08$ & 19.4211 \\
G8L3 & $4.7155 \mathrm{E}-10$ & 15.8206 & $3.3960 \mathrm{E}-10$ & 15.6800 & $1.0623 \mathrm{E}-09$ & 17.1218 \\
\hline
\end{tabular}

Tabela 5.32: Erro e ordem de convergência para as componentes do tensor viscoelástico com Modelo FENE$P$ para $l=7, W_{i}=10$ e $t=0.25$.

Na discretização do tensor $\mathbf{S}$, tanto com o Modelo Oldroyd-B como com o Modelo FENE-P, a razão de convergência com na norma do máximo tende à quarta ordem, quando o esperado seria segunda ordem.

\subsection{Verificação das equações de Navier-Stokes (escoamento incom- pressivel transiente)}

\subsubsection{Fluido newtoniano monofásico}

Para verificar a implementação das equações de Navier-Stokes, lembramos que empregamos o Método da Projeção de Chorin-Temam e o método multinível-multigrid pertencentes ao AMR2D 
original. Além disso, a discretização temporal original empregava o Método de Gear, para passo de tempo variável, e o Método de Crank-Nicolson Modificado (MCNAB) com passo de tempo constante. Tais esquemas de discretização no tempo foram substituídos por um esquema paramétrico do tipo implícito-explícito (IMEX) geral com passo de tempo variável. Mostramos aqui um teste que emprega passo de tempo constante usando os parâmetros $(c, \gamma)$ com $\left(\frac{1}{8}, \frac{1}{2}\right)$ e $\left(0, \frac{1}{2}\right)$, isto é, os métodos de Crank-Nicolson Modificado e de Crank-Nicolson Adams-Bashforth, MCNAB e CNAB, respectivamente. Verificamos a convergência e a ordem esperada empregando as funções

$$
\begin{aligned}
p(x, y ; t) & =\cos (2 \pi x) \cos (2 \pi y), \\
u(x, y ; t) & =-2 \cos (\pi t) \sin ^{2}(\pi x) \cos (\pi y) \sin (\pi y), \\
v(x, y ; t) & =2 \cos (\pi t) \cos (\pi x) \sin (\pi x) \sin ^{2}(\pi y), \\
\nabla \cdot \mathbf{u}=0, \quad \mu & =1, \quad \rho=1, \quad t_{f}=1.0 \quad \Omega=[0,1] \times[0,1],
\end{aligned}
$$

com condição de contorno periódica na direção $x$ e de Dirichlet na outra direção para u e homogênea de Neumann para $p$.

Na Tabela 5.33, mostramos os resultados obtidos usando a malha composta 5.1 com três níveis e com o nível base dado como união de retalhos, usando os esquemas MCNAB e CNAB na discretização temporal. Obtivemos ordem de convergência para ambas as direções da velocidade tendendo a segunda ordem e para a pressão tendendo a $\log _{2}(3.5) \approx 1.807$. Nesta tabela, também mostramos a quantidade de passos temporais para obter a solução onde $\Delta t$ deve satisfazer a mesma condição que o tensor para a equação de advecção $\Delta t \leq \frac{1}{\Delta t_{u}+\Delta t_{v}}$.

\begin{tabular}{ccccccccc}
\hline IMEX & malha & nstep & $\|e(u)\|_{2}$ & $r_{2} u$ & $\|e(v)\|_{2}$ & $r_{2} v$ & $\|e(p)\|_{2}$ & $r_{2} p$ \\
\hline MCNAB & G4L3 & 320 & $5.4993 \mathrm{E}-04$ & & $2.4493 \mathrm{E}-04$ & & $2.6454 \mathrm{E}-02$ & \\
& G5L3 & 640 & $1.3822 \mathrm{E}-04$ & 3.9788 & $6.0762 \mathrm{E}-05$ & 4.0310 & $7.2267 \mathrm{E}-03$ & 3.6605 \\
& G6L3 & 1280 & $3.4418 \mathrm{E}-05$ & 4.0158 & $1.5220 \mathrm{E}-05$ & 3.9921 & $1.9304 \mathrm{E}-03$ & 3.7437 \\
& G7L3 & 2560 & $8.6010 \mathrm{E}-06$ & 4.0017 & $3.8133 \mathrm{E}-06$ & 3.9914 & $5.2697 \mathrm{E}-04$ & 3.6632 \\
& G8L3 & 5120 & $2.1504 \mathrm{E}-06$ & 3.9997 & $9.5463 \mathrm{E}-07$ & 3.9946 & $1.4908 \mathrm{E}-04$ & 3.5348 \\
\hline CNAB & G4L3 & 320 & $3.6822 \mathrm{E}-04$ & & $2.5465 \mathrm{E}-04$ & & $1.9163 \mathrm{E}-02$ & \\
& G5L3 & 640 & $9.4604 \mathrm{E}-05$ & 3.8923 & $6.4704 \mathrm{E}-05$ & 3.9356 & $5.1022 \mathrm{E}-03$ & 3.7559 \\
& G6L3 & 1280 & $2.4079 \mathrm{E}-05$ & 3.9289 & $1.6194 \mathrm{E}-05$ & 3.9956 & $1.3765 \mathrm{E}-03$ & 3.7067 \\
& G7L3 & 2560 & $6.0816 \mathrm{E}-06$ & 3.9593 & $4.0442 \mathrm{E}-06$ & 4.0041 & $3.8906 \mathrm{E}-04$ & 3.5379 \\
& G8L3 & 5120 & $1.5288 \mathrm{E}-06$ & 3.9779 & $1.0104 \mathrm{E}-06$ & 4.0027 & $1.1584 \mathrm{E}-04$ & 3.3586 \\
\hline
\end{tabular}

Tabela 5.33: Erro e ordem para cada direção da velocidade e a pressão usando o IMEX com parâmetros $\left(\frac{1}{8}, \frac{1}{2}\right)$ e $\left(0, \frac{1}{2}\right)$, na solução das equações de Navier-Stokes.

Com as outras opções de parâmetros para o IMEX, obtivemos resultados na mesma ordem e precisão dos mostrados na Tabela 5.33 .

\subsubsection{Fluido não newtoniano monofásico}

Verificamos a aproximação da solução das equações de Navier-Stokes quando $\alpha_{p} \neq 0$ nas equações (2.1)-(2.2), isto é, quando o tensor viscoelástico é diferente de zero e as equações modelam um escoamento viscoelástico. Empregamos o Modelo Oldroyd-B para o tensor viscoelástico. Na solução manufaturada, usamos para a velocidade, para a pressão, para a massa específica e para a viscosidade as funções (5.36) empregadas anteriormente no teste para fluido newtoniano 
monofásico. Para o tensor viscoelástico, empregamos as funções

$$
\begin{aligned}
& S_{x x}(x, y ; t)=-2 \cos (\pi t) \sin ^{2}(\pi x) \cos (\pi y) \sin (\pi y), \\
& S_{x y}(x, y ; t)=t \sin (2 \pi x) \sin (2 \pi y), \\
& S_{y y}(x, y ; t)=e^{-t} \sin (2 \pi x) \sin (2 \pi y) .
\end{aligned}
$$

O teste é para condição de contorno mista, da mesma forma como foi feito no caso newtoniano, usando na discretização temporal o esquema MCNAB e os resultados obtidos estão na Tabela 5.34. No caso das velocidades, a convergência aproxima-se de segunda ordem e para a pressão aproximase de $\log _{2}(3.1) \approx 1.632$. Para o tensor viscoelástico, mostramos apenas a componente $S_{x x}$ dado que o comportamento para as outras componentes tensoriais foi semelhante e a ordem ficou entre terceira e quarta ordem. A quantidade de passos no tempo são para a aproximação da equação de Navier-Stokes.

\begin{tabular}{cccccccccc}
\hline malha & nstep & $\|e(u)\|_{2}$ & $r_{2} u$ & $\|e(v)\|_{2}$ & $r_{2} v$ & $\|e(p)\|_{2}$ & $r_{2} p$ & $S_{x x}$ & $r_{2} S_{x x}$ \\
\hline G4L3 & 320 & $6.3133 \mathrm{E}-04$ & & $5.4123 \mathrm{E}-04$ & & $2.6454 \mathrm{E}-02$ & & $7.3367 \mathrm{E}-03$ & \\
G5L3 & 640 & $1.5241 \mathrm{E}-04$ & 4.1423 & $1.2798 \mathrm{E}-04$ & 4.2291 & $8.5412 \mathrm{E}-03$ & 3.0972 & $5.0344 \mathrm{E}-04$ & 14.5732 \\
G6L3 & 1280 & $3.5239 \mathrm{E}-05$ & 4.3250 & $3.0615 \mathrm{E}-05$ & 4.1802 & $2.7205 \mathrm{E}-03$ & 3.1396 & $3.3848 \mathrm{E}-05$ & 14.8732 \\
G7L3 & 2560 & $9.1126 \mathrm{E}-06$ & 3.8671 & $6.4687 \mathrm{E}-06$ & 4.7328 & $8.7351 \mathrm{E}-04$ & 3.1144 & $2.4338 \mathrm{E}-06$ & 13.9075 \\
G8L3 & 5120 & $2.1650 \mathrm{E}-06$ & 4.2091 & $1.4676 \mathrm{E}-06$ & 4.4076 & $2.8336 \mathrm{E}-04$ & 3.0827 & $1.6461 \mathrm{E}-07$ & 14.7854 \\
\hline
\end{tabular}

Tabela 5.34: Erro e ordem de convergência na solução das equações de Navier-Stokes viscoelásticas.

O caso bifásico para a solução das equações de Navier-Stokes para um fluido newtoniano foi apresentado por Villar [111]. O caso não newtoniano será abordado em um trabalho futuro.

\subsection{Verificação das equações de Stokes (escoamento incompressivel estacionário)}

\subsubsection{Fluido newtoniano monofásico}

Apresentamos nesta seção a aproximação obtida para as equações de Stokes de um escoamento incompressível estacionário (2.41)- (2.44). Resolvemos as equações discretizadas empregando o Método de Uzawa em conjunto com o Método BiCG-Stab, discutido na Seção 3.5. Usamos o Algoritmo 2 (SAMR Método de Uzawa com BiCG-Stab retalho-a-retalho), dado na Seção 4.3.

Solucionamos a seguir as equações discretas (3.62)- (3.63) para $\mu=1$ constante, usando a solução manufaturada com $\mathbf{f}=-\nabla p+\nabla^{2} \mathbf{u}$ e as funções

$$
\begin{aligned}
p(x, y) & =\sin (2 \pi x) \sin (2 \pi y), \\
u(x, y) & =-2 \sin ^{2}(\pi x) \cos (\pi y) \sin (\pi y), \\
v(x, y) & =2 \cos (\pi x) \sin (\pi x) \sin ^{2}(\pi y), \\
\nabla \cdot \mathbf{u} & =0 \quad \Omega=[0,1] \times[0,1] .
\end{aligned}
$$


Na Figura 5.18, temos a malha composta com dois níveis que empregamos no problema. Tal malha possui dez retalhos: três no nível de base e sete no segundo nível. Usamos condição de contorno mista, onde temos condição periódica na direção $x$ e de Dirichlet na outra direção para a velocidade e homogênea de Neumann para a pressão.

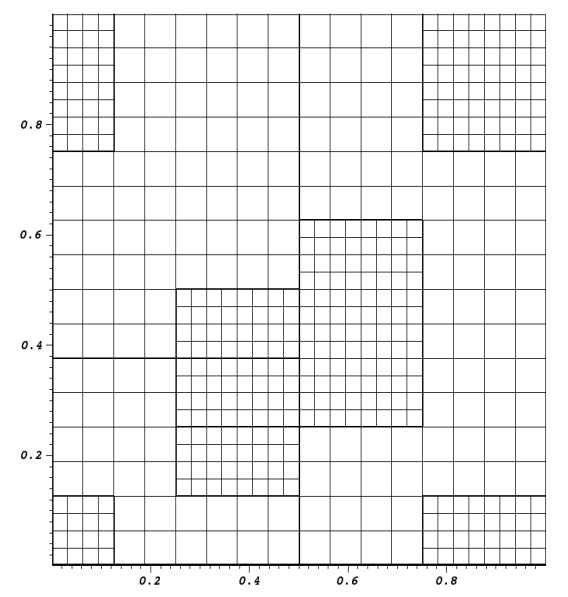

Figura 5.18: Malha composta empregada na verificação da equação de Stokes para um escoamento incompressivel estacionário.

Os resultados obtidos estão na Tabela 5.35 e podemos constatar que na norma $L 2$ todas as aproximações são de segunda ordem. Entretanto, na norma infinito as velocidades tem segunda ordem mas a pressão tem ordem um.

\begin{tabular}{ccccccc}
\hline Malha & $\|e(p)\|_{2}$ & $r_{2} p$ & $\|e(u)\|_{2}$ & $r_{2} u$ & $\|e(v)\|_{2}$ & $r_{2} v$ \\
\hline G4L2 & $1.4003 \mathrm{E}-02$ & & $4.5701 \mathrm{E}-03$ & & $4.2675 \mathrm{E}-03$ & \\
G5L2 & $3.5333 \mathrm{E}-03$ & 3.9630 & $1.1436 \mathrm{E}-03$ & 3.9961 & $1.0731 \mathrm{E}-03$ & 3.9767 \\
G6L2 & $8.8933 \mathrm{E}-04$ & 3.9730 & $2.8686 \mathrm{E}-04$ & 3.9867 & $2.6995 \mathrm{E}-04$ & 3.9752 \\
G7L2 & $2.2303 \mathrm{E}-04$ & 3.9875 & $7.1899 \mathrm{E}-05$ & 3.9898 & $6.7752 \mathrm{E}-05$ & 3.9844 \\
\hline & $\|e(p)\|_{\infty}$ & $r_{\infty} p$ & $\|e(u)\|_{\infty}$ & $r_{\infty} u$ & $\|e(v)\|_{\infty}$ & $r_{\infty} v$ \\
\hline G4L2 & $9.0150 \mathrm{E}-02$ & & $1.0246 \mathrm{E}-02$ & & $1.0425 \mathrm{E}-02$ & \\
G5L2 & $4.2353 \mathrm{E}-02$ & 2.1285 & $2.5805 \mathrm{E}-03$ & 3.9707 & $2.5641 \mathrm{E}-03$ & 4.0659 \\
G6L2 & $2.1696 \mathrm{E}-02$ & 1.9521 & $6.5147 \mathrm{E}-04$ & 3.9611 & $6.4412 \mathrm{E}-04$ & 3.9808 \\
G7L2 & $1.1042 \mathrm{E}-02$ & 1.9648 & $1.6380 \mathrm{E}-04$ & 3.9772 & $1.6134 \mathrm{E}-04$ & 3.9923 \\
\hline
\end{tabular}

Tabela 5.35: Erro e ordem de convergência na solução das equações de Stokes para um escoamento incompressivel estacionário monofásico.

Neste teste, para a solução do sistema linear proveniente da discretização do laplaciano em meio de arestas, empregamos a representação matricial e a biblioteca PETSc com o Método GMRES com precondicionador ILU(0) e tolerância do resíduo de $10^{-12}$. Mostramos na Tabela 5.36 a quantidade de iterações do Algoritmo BiCG-Stab para cada malha composta e o divergente obtido para a velocidade na iteração final do BiCG-Stab (note que este divergente coincide com o valor do resíduo final nas iterações do BiCG-Stab). 


\begin{tabular}{ccc}
\hline Malha & Iter(BiCG-Stab) & $\|\nabla \cdot \mathbf{u}\|_{2}$ \\
\hline G4L2 & 11 & $1.28 \mathrm{E}-011$ \\
G5L2 & 12 & $1.11 \mathrm{E}-012$ \\
G6L2 & 12 & $2.71 \mathrm{E}-011$ \\
G7L2 & 13 & $7.51 \mathrm{E}-012$ \\
\hline
\end{tabular}

Tabela 5.36: Número de iterações do Método BiCG-Stab para cada malha composta empregada e norma do divergente da velocidade.

\subsubsection{Fluido newtoniano bifásico}

Para este teste simulamos a deformação sofrida por uma gota circular sob a ação de um escoamento cisalhante, dado por $\mathbf{u}=[\Upsilon y, 0]^{T}$, onde $\Upsilon$ é a velocidade de cisalhamento que neste caso a tomamos constante e igual à tensão superficial $\sigma$ e a condição de contorno para todas as variáveis é periódica na direção horizontal e de Dirichlet ao norte e ao sul do domínio computacional. Usamos neste teste $\mu=1, \rho=1, \sigma=0.005524$ e $\Omega=[0,1] \times[0,1]$. Iniciando com pressão zero, as componentes da velocidade e a pressão são determinadas pelo Método adaptativo de Uzawa BiCG-Stab no instante inicial. A força da fronteira imersa foi determinada usando a formulação híbrida disponível na implementação AMR2D.

Escolhemos este teste por que futuramente ele nós permitirá fazer comparações físicas, especialmente quando incluirmos o tensor viscoelástico (escoamento bifásico não newtoniano).

Os resultados (parciais) mostram que o comportamento geométrico de deformação esperado foi obtido, entretanto ainda é necessária uma análise da relação entre a capilaridade e a deformação.

Empregamos uma malha composta que denominamos "G7L3" formada por uma malha de base com $128 \times 128$ células computacionais e três níveis de refinamento. A remalhagem ocorre a cada quatro passos no tempo. Na Figura 5.19, temos as malhas compostas empregadas nos instantes de tempo 0,60, 152 e 284. Para cada um destes instantes a quantidade de retalhos da malha é 114, 104, 100 e 107, respectivamente.

\subsubsection{Fluido não newtoniano monofásico}

A verificação para a malha composta neste caso é feita usando a solução manufaturada para as equações de Stokes (3.62)- (3.70) modelando um escoamento de fluido viscoelástico, usando o Método de Uzawa BiCG-Stab na malha composta.

Usamos a força manufaturada $\mathbf{f}^{\text {man }}$ dada por

$$
\mathbf{f}^{\text {man }}=-\mathbf{D} \cdot \mathbf{S}+\mathbf{f}
$$

onde $\mathbf{f}=-\nabla p+\nabla^{2} \mathbf{u}$ e solucionamos as equações de Stokes em um escoamento estacionário de um fluido viscoelástico, dadas por

$$
-\nabla p+\nabla^{2} \mathbf{u}=\mathbf{f}^{\text {man }} \quad \text { e } \quad \nabla \cdot \mathbf{u}=0
$$



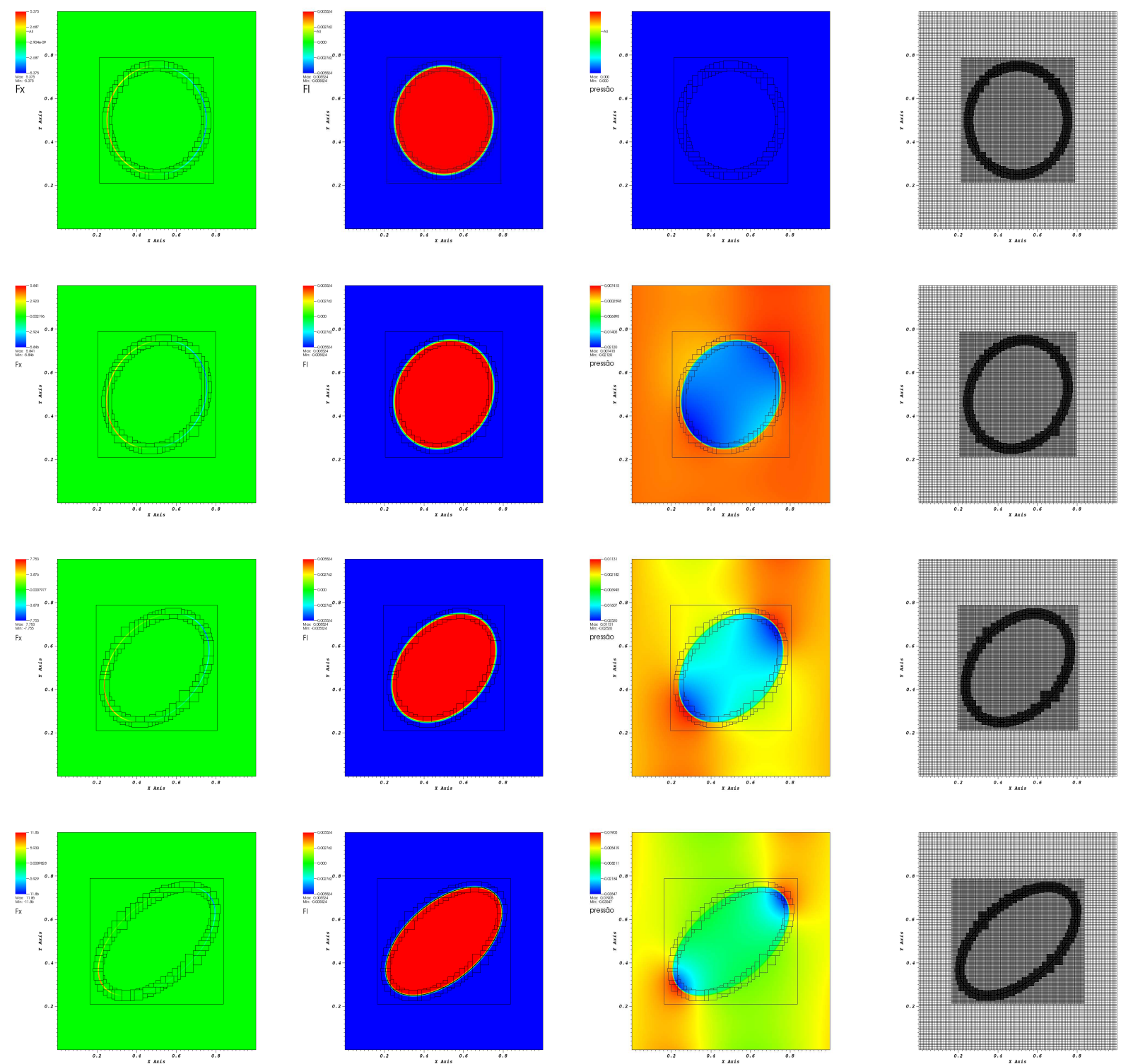

Figura 5.19: Aproximações obtidas com a solução da equação de Stokes para um escoamento incompressivel estacionário de um fluido bifásico a baixo cisalhamento. Da esquerda à direta, temos a força $f_{x}$ dada pela interface na aresta vertical, a função indicadora, a pressão e a malha composta em cada caso. 
onde as funções para a velocidade e a pressão são dadas em (5.37) e para o tensor viscoelástico são

$$
\begin{aligned}
& S_{x x}=e^{-t} \sin (2 \pi x) \sin (2 \pi y), \\
& S_{x y}=t \sin (2 \pi x) \sin (2 \pi y), \\
& S_{y y}=e^{-t} \sin (2 \pi x) \sin (2 \pi y) .
\end{aligned}
$$

Na Tabela 5.37, mostramos os resultados obtidos neste caso, no qual empregamos o Modelo OldroydB para o tensor viscoelástico e usamos uma malha composta dada por dois níveis de refinamento, mostrada na Figura 5.18. Na norma infinito, conseguimos convergência de segunda ordem para ambas as componentes da velocidade e primeira ordem para a pressão, como já havia acontecido na verificação das equações de Stokes de um fluido monofásico newtoniano (Tabela 5.35). Incluímos também na Tabela 5.37 a quantidade de iterações do Método BiCG-Stab e a norma infinito do resíduo final. A solução do sistema linear com a biblioteca PETSc é novamente baseada no Método GMRES com ILU(0) como pré-condicionador.

\begin{tabular}{ccccccccc}
\hline Malha & $\|e(u)\|_{\infty}$ & $r_{\infty} u$ & $\|e(v)\|_{\infty}$ & $r_{\infty} v$ & $\|e(p)\|_{\infty}$ & $r_{\infty} p$ & Iter(BCGS) & $\|r e s\|_{\infty}$ \\
\hline G4L2 & $7.7228 \mathrm{E}-03$ & & $7.7228 \mathrm{E}-03$ & & $1.1472 \mathrm{E}-01$ & & 8 & $6.2004 \mathrm{E}-13$ \\
G5L2 & $2.4464 \mathrm{E}-03$ & 3.1568 & $2.4464 \mathrm{E}-03$ & 3.1568 & $5.5417 \mathrm{E}-02$ & 2.0700 & 9 & $3.7493 \mathrm{E}-13$ \\
G6L2 & $7.0561 \mathrm{E}-04$ & 3.4671 & $7.0520 \mathrm{E}-04$ & 3.4691 & $2.7095 \mathrm{E}-02$ & 2.0453 & 9 & $1.8345 \mathrm{E}-13$ \\
G7L2 & $1.8969 \mathrm{E}-04$ & 3.7198 & $1.9060 \mathrm{E}-04$ & 3.6998 & $1.3281 \mathrm{E}-02$ & 2.0401 & 10 & $9.7247 \mathrm{E}-13$ \\
\hline
\end{tabular}

Tabela 5.37: Erro e razão de convergência com a solução manufatura da equação de Stokes com o termo viscoelástico. 


\section{Capítulo 6}

\section{Conclusão e perspectivas futuras}

Motivados pelo estudo da dinâmica de escoamentos incompressíveis bifásicos de fluidos imiscíveis em regimes transiente e estacionário, principalmente no contexto de aplicações nas quais pode haver uma fase viscoelástica (uma fase não newtoniana), apresentamos em detalhes modelos matemáticos e métodos numéricos capazes de realizar de maneira robusta e eficiente as respectivas simulações computacionais. Os métodos de Um Fluido [32, 105, 106, 107] e da Fronteira Imersa [48, 81, 85] aliados aos métodos da Projeção de Chorin-Temam [34, 97] e o de Uzawa [4, 7, 56, 57] (este último aplicado em conjunto com o Método do Gradiente Biconjugado Estabilizado BiCG-Stab [13, 57, 108]), compõem a seleção escolhida para abordar a resolução numérica das equações obtidas dos modelos matemáticos mencionados.

Mostramos como a aplicação de cada combinação de métodos depende da resolução de uma série de problemas-modelo essencialmente mais simples baseados em equações diferenciais parciais elípticas e/ou parabólicas. Mais especificamente, a aplicação do Método da Projeção de ChorinTemam para resolver escoamentos incompressíveis (bifásicos) transientes, depende da resolução de uma equação parabólica para encontrar um campo de velocidades preliminar que, para ser projetado no espaço dos campo vetoriais com divergente zero, depende da resolução de uma equação elíptica para a correção de pressão. No caso de escoamentos estacionários, mostramos como o Método de Uzawa envolve a resolução de equações elípticas para as componentes da velocidade e um sistema linear para a pressão, resultante do processo que desacopla a pressão da velocidade - este último sistema é resolvido de maneira iterativa usando o Método BiCG-Stab.

Os problemas-modelo essenciais, discretizados em malhas compostas bloco-estruturadas SAMR, dão origem a sistemas lineares os quais foram resolvidos empregando-se uma biblioteca numérica externa, no caso, o PETSc [11]. O núcleo de nosso trabalho foi exatamente propor algoritmos e estruturas de dados que viabilizassem a obtenção explícita das matrizes que representam em cada caso a discretização de um dos modelos de interesse em malhas compostas. A obtenção de tais matrizes representando as discretizações passa pela obtenção dos pesos usados na interpolação de valores fantasmas, necessários à implementação dos esquemas de diferenças finitas empregados, e também calculados usando formas matriciais explícitas.

A obtenção explícita de tais matrizes no contexto de malhas compostas adaptativas blocoestruturadas e do Método das Diferenças Finitas é um problema pouquíssimo estudado, havendo virtualmente nenhum relato a seu respeito. Tal abordagem matricial constitui, em nossa opinião, um contribuição importante pois ela permite, efetivamente, caracterizar as propriedades dos sistemas lineares obtidos durante as discretizações dos problemas-modelos essenciais mencionados: seus 
condicionamentos, raios espectrais, como se comportam os autovalores, propriedades de definição e simetria. Além disso, tendo-se acesso à forma matricial explícita, ganhamos acesso ao uso de vários métodos existentes e disponíveis inclusive em versões paralelas, via uso de bibliotecas numéricas externas. Merece especial destaque que, com as matrizes explicitamente calculadas, podemos usar pré-condicionadores para resolver os sistemas lineares associados de maneira mais eficiente.

Ao longo do processo de obtenção das matrizes mencionadas anteriormente, conseguimos identificar, em várias oportunidades, formas de aprimorar e estender as subrotinas responsáveis por cálculos de valores fantasmas do AMR2D [85, 110, 111], baseadas em algoritmos "naturais" que dependem de visitas retalho-a-retalho e de varreduras nível-a-nível da hierarquia de malhas. O contínuo aprimoramento de tais subrotinas permitiu que o Método BiCG-Stab (usado no Método de Uzawa) pudesse ser implementado dentro deste paradigma mais natural no contexto SAMR. Consideramos este aprimoramento e implementação uma grande contribuição de nosso trabalho.

Uma vez tendo obtido sucesso na resolução matricial dos problemas-modelo essenciais que compõem os métodos da Projeção de Chorin-Temam e de Uzawa, podemos usá-los em conjunto para simular computacionalmente as aplicações almejadas. Neste sentido, alguns primeiros passos forma dados. Estendemos o AMR2D de maneira que ele agora pode conter uma fase viscoelástica. Essa extensão depende da resolução da equação hiperbólica que nos fornece o tensor de tensões viscoelásticas (no momento, via modelos Oldroyd-B ou FENE-P). Esta implementação é baseada no uso de esquemas ENO de terceira ordem e de Runge-Kutta TVD de segunda ordem em malhas compostas.

Ao empregarmos para solucionar a equação de Poisson, oriunda da correção da pressão no Método da Projeção, o método multinível-multigrid em problemas bifásicos com altas razões de massa específica, foram encontrados problemas com a convergência do método [111]. Na Seção 5.3.1 relatamos testes que comparam a resolução desta equação usando o método multinível-multigrid e a representação matricial da discretização com diferentes solvers e pré-condicionadores do PETSc. Com o uso da matriz e do PETSc para altas razões conseguimos obter convergência em casos onde o método multinível-multigrid não conseguiu, e ao usar paralelismo, obtivemos para razões moderadas um tempo de resolução inferior ao obtido com o multinível-multigrid.

Na Figura 6.1, apresentamos a nossa perspectiva de trabalhos futuros a curto (em cor cinza) e a longo prazo (em cor branco). Para a finalização dos blocos na cor cinza, precisamos solucionar um problema de transferência de dados ao usarmos malhas dinâmicas que mudam com propriedades da interface ou específicas do problema. Este problema é devido ao fato de que precisamos de uma camada de três células fantasmas ao redor de cada retalho e o AMR2D foi projetado para ser usado com uma camada só. Uma vez resolvido este problema, iremos fazer aplicações com poucas bolhas ou gotículas, combinando fases newtonianas e viscoelásticas, tanto para baixo Reynolds como para Reynolds zero.

Os itens em cor branco precisam da implementação de versões eficientes do Método da Fronteira Imersa [26, 27, 28], pois pensamos em problemas com um número grande de interfaces imersas (e.g. borbulhamento em polímeros ou grande número de gotículas no escoamento). Para tal, precisamos conseguir versões da metodologia com restrições temporais mais brandas e preparadas para lidar melhor com um grande número de pontos lagrangianos e suas interações. 


\begin{tabular}{|c|c|}
$\begin{array}{c}S A M R \text { Uzawa } \\
\text { Stokes } \\
\text { bifásico (viscoelástico) }\end{array}$ & $\begin{array}{c}\text { SAMR Chorin-Temam } \\
\text { Navier-Stokes } \\
\text { bifásico (viscoelástico) }\end{array}$ \\
\hline $\begin{array}{c}\text { SAMR Chorin-Temam } \\
\text { Navier-Stokes } \\
\text { "escoamento complexo" }\end{array}$ & $\begin{array}{c}\text { SAMR Uzawa } \\
\text { Stokes } \\
\text { "escoamento complexo" }\end{array}$ \\
\hline
\end{tabular}

Figura 6.1: Cinza: trabalho futuro a curto prazo. Branco: trabalho futuro a longo prazo. 


\section{Apêndice A}

\section{Adimensionalização}

Seja $\mathbf{S}_{p}$ o tensor polimérico responsável pela contribuição oriunda da fase viscoelástica (fase não newtoniana) e $\mathbf{S}_{N}$ o tensor newtoniano dado por

$$
\mathbf{S}_{N}=-p I+2 \mu \mathbf{D}
$$

sendo $p$ o campo de pressão, $\mu$ a viscosidade dinâmica do fluido, $\mathbf{D}$ o tensor deformação e $\mathbf{D}=$ $\frac{1}{2}\left(\nabla \mathbf{u}+\nabla \mathbf{u}^{\mathbf{T}}\right)$ com $\mathbf{u}=(u, v)$ representando o campo de velocidade.

Desta forma, as equações de Navier-Stokes que modelam escoamentos incompressíveis de fluidos newtonianos (ou não newtonianos) são dadas por

$$
\begin{aligned}
& \rho \frac{D \mathbf{u}}{D t}=\nabla \cdot\left(\mathbf{S}_{N}+\mathbf{S}_{p}\right)+\mathbf{f}, \\
& \nabla \cdot \mathbf{u}=0 \quad \text { em } \Omega
\end{aligned}
$$

onde $\rho$ é a massa específica do fluido, $\mathbf{f}$ são forças que atuam no fluido, $\Omega$ é o domínio e o operador $D$ é a derivada total definida por

$$
D=\frac{\partial}{\partial t}+\mathbf{u} \cdot \nabla
$$

Usando a definição anterior em (A.1), temos que

$$
\rho\left(\frac{\partial \mathbf{u}}{\partial t}+\mathbf{u} \cdot \nabla\right)=-\nabla p+\nabla\left[\mu\left(\nabla \mathbf{u}+\nabla \mathbf{u}^{\mathbf{T}}\right)\right]+\nabla \cdot \mathbf{S}_{p}+\mathbf{f} .
$$

Muitas abordagens diferentes têm sido empregadas na modelagem do tensor polimérico. No presente trabalho, foram empregados os modelos Oldroyd-B e FENE-P. Para mostrar o procedimento adotado na adimensionalização, usaremos o Modelo Oldroyd-B

$$
\tau \mathbf{S}_{p}^{\nabla}=-\left(\mathbf{S}_{p}-G I\right), \quad \text { em } \Omega
$$

onde $\tau$ é a escala do tempo para a relação polimérica, $G$ é o estresse isotrópico induzido pelo campo polimérico na ausência de fluido e

$$
\mathbf{S}_{p}^{\nabla}=\frac{\partial \mathbf{S}_{p}}{\partial t}+\mathbf{u} \cdot \nabla \mathbf{S}_{p}-\left(\nabla \mathbf{u} \mathbf{S}_{p}+\mathbf{S}_{p} \nabla \mathbf{u}^{T}\right)
$$

é a derivada convectada superior. 
Algumas propriedades importantes de escoamentos podem ser caracterizadas ao serem adimensionalizadas as equações que os definem.

$\mathrm{Na}$ adimensionalização de (A.1)-(A.4), tomemos

$$
\overline{\mathbf{u}}=\frac{\mathbf{u}}{U_{c}}, \quad \overline{\mathbf{x}}=\frac{\mathbf{x}}{L_{c}}, \quad \bar{t}=\frac{t}{t_{c}}, \bar{p}=\frac{p L_{c}}{\mu_{c} U_{c}}, \bar{\mu}=\frac{\mu}{\mu_{c}}, \quad \bar{\rho}=\frac{\rho}{\rho_{c}}, \quad \text { e } \quad \overline{\mathbf{S}}_{p}=\frac{1}{G} \mathbf{S}_{p},
$$

onde $\overline{\mathbf{u}}=(\bar{u}, \bar{v}), \overline{\mathbf{x}}=(\bar{x}, \bar{y})$ e $U_{c}, L_{c}, \tau, \mu_{c}, \rho_{c}$ e $G$ são valores típicos que caracterizam cada uma das variáveis. No nosso caso, $L_{c}$ é o comprimento característico dado pelo comprimento do domínio $\left(L_{c}=L\right), U_{c}$ é a velocidade característica, $t_{c}$ é o tempo característico requerido para o fluido ser transportado a uma distância da ordem do comprimento do domínio $\left(t_{c}=\frac{L}{U_{c}}\right)$ e $\mu_{c}$ é a viscosidade característica.

No que segue, usaremos a variável $\phi$ para representar qualquer uma das variáveis anteriores e $\phi_{c}$ é o valor característico associado a $\phi$. Por exemplo, se $\phi=\mathbf{S}_{p}$, temos $\phi_{c}=G$.

Se denotarmos $\nabla^{\prime}=\left(\frac{\partial}{\partial \bar{x}}, \frac{\partial}{\partial \bar{y}}\right)$, teremos que:

1. O gradiente adimensional aplicado a $\phi$ é dado por

$$
\begin{aligned}
\nabla \phi & =\left(\frac{\partial \phi}{\partial x}, \frac{\partial \phi}{\partial y}\right) \\
& =\left(\frac{\partial\left(\phi_{c} \bar{\phi}\right)}{\partial\left(L_{c} \bar{x}\right)}, \frac{\partial\left(\phi_{c} \bar{\phi}\right)}{\partial\left(L_{c} \bar{y}\right)}\right) \\
& =\frac{\phi_{c}}{L_{c}}\left(\frac{\partial \bar{\phi}}{\partial \bar{x}}, \frac{\partial \bar{\phi}}{\partial \bar{y}}\right) \\
& =\frac{\phi_{c}}{L_{c}} \nabla^{\prime} \bar{\phi}
\end{aligned}
$$

2. O Laplaciano adimensional aplicado a $\phi$ é

$$
\nabla^{2} \phi=\frac{\phi_{c}}{L_{c}^{2}} \nabla^{\prime 2} \bar{\phi}
$$

3. A derivada total adimensional aplicada a $\phi$ é

$$
\begin{aligned}
\frac{D \phi}{D t} & =\frac{\partial \phi}{\partial t}+\mathbf{u} \cdot \nabla \phi \\
& =\frac{\phi_{c}}{t_{c}} \frac{\partial \bar{\phi}}{\partial \bar{t}}+\frac{U_{c} \phi_{c}}{L_{c}} \overline{\mathbf{u}} \cdot \nabla^{\prime} \bar{\phi} \\
& =\frac{U_{c} \phi_{c}}{L_{c}} \frac{\partial \bar{\phi}}{\partial \bar{t}}+\frac{U_{c} \phi_{c}}{L_{c}} \overline{\mathbf{u}} \cdot \nabla^{\prime} \bar{\phi} \\
& =\frac{U_{c} \phi_{c}}{L_{c}}\left(\frac{\partial \bar{\phi}}{\partial \bar{t}}+\overline{\mathbf{u}} \cdot \nabla^{\prime} \bar{\phi}\right) \\
& =\frac{U_{c} \phi_{c}}{L_{c}} \frac{D \bar{\phi}}{D \bar{t}} ;
\end{aligned}
$$


4. A derivada convectada superior adimensional aplicado a $\phi$ é dada por

$$
\begin{aligned}
\phi^{\nabla} & =\frac{\phi_{c}}{t_{c}} \frac{\partial \bar{\phi}}{\partial \bar{t}}+\frac{U_{c} \phi_{c} \overline{\mathbf{u}}}{L_{c}} \cdot \nabla^{\prime} \bar{\phi}-\left(\frac{U_{c} \phi_{c}}{L_{c}} \nabla^{\prime} \overline{\mathbf{u}} \bar{\phi}+\frac{U_{c} \phi_{c}}{L_{c}} \bar{\phi} \nabla^{\prime} \overline{\mathbf{u}}^{T}\right) \\
& =\frac{\phi_{c}}{t_{c}} \frac{\partial \bar{\phi}}{\partial \bar{t}}+\frac{\phi_{c}}{t_{c}} \overline{\mathbf{u}} \cdot \nabla^{\prime} \bar{\phi}-\frac{\phi_{c}}{t_{c}}\left(\nabla^{\prime} \overline{\mathbf{u}} \bar{\phi}+\bar{\phi} \nabla^{\prime} \overline{\mathbf{u}}^{T}\right) \\
& =\frac{\phi_{c}}{t_{c}}\left(\frac{\partial \phi}{\partial \bar{t}}+\overline{\mathbf{u}} \cdot \nabla^{\prime} \phi-\left(\nabla^{\prime} \overline{\mathbf{u}} \phi+\phi \nabla^{\prime} \overline{\mathbf{u}}^{T}\right)\right) \\
& =\frac{\phi_{c}}{t_{c}} \phi^{\nabla^{\prime}} .
\end{aligned}
$$

Em consequência, ao adimensionalizar a equação (A.1) obtemos

$$
\begin{aligned}
\bar{\rho} \rho_{c} \frac{U_{c}}{\tau_{c}} \frac{D \overline{\mathbf{u}}}{D \bar{t}} & =-\frac{\mu_{c} U_{c}}{L_{c}^{2}} \nabla^{\prime} \bar{p}+\nabla \cdot\left(\frac{\mu_{c} U_{c}}{L_{c}} \bar{\mu}\left(\nabla^{\prime} \overline{\mathbf{u}}+\nabla^{\prime} \overline{\mathbf{u}}^{T}\right)\right)+\frac{G}{L_{c}} \nabla^{\prime} \cdot \overline{\mathbf{S}}_{p}+\mathbf{f} \\
& =-\frac{\mu_{c} U_{c}}{L_{c}^{2}} \nabla^{\prime} \bar{p}+\frac{\mu_{c} U_{c}}{L_{c}^{2}} \nabla^{\prime}\left(\bar{\mu}\left(\nabla^{\prime} \overline{\mathbf{u}}+\nabla^{\prime} \overline{\mathbf{u}}^{T}\right)\right)+\frac{G}{L_{c}} \nabla^{\prime} \cdot \overline{\mathbf{S}}_{p}+\mathbf{f} \\
& =\frac{\mu_{c} U_{c}}{L_{c}^{2}}\left[-\nabla^{\prime} \bar{p}+\nabla^{\prime}\left(\bar{\mu}\left(\nabla^{\prime} \overline{\mathbf{u}}+\nabla^{\prime} \overline{\mathbf{u}}^{T}\right)\right)+\frac{G L_{c}}{\mu_{c} U_{c}} \nabla^{\prime} \cdot \overline{\mathbf{S}}_{p}+\frac{L_{c}^{2} \mathbf{f}}{\mu_{c} U_{c}}\right] \\
& =\frac{\mu_{c}}{L_{c} \tau_{c}}\left[-\nabla^{\prime} \bar{p}+\nabla^{\prime}\left(\bar{\mu}\left(\nabla^{\prime} \overline{\mathbf{u}}+\nabla^{\prime} \overline{\mathbf{u}}^{T}\right)\right)+\frac{G \tau_{c}}{\mu_{c}} \nabla^{\prime} \cdot \overline{\mathbf{S}}_{p}+\frac{L_{c}^{2} \mathbf{f}}{\mu_{c} U_{c}}\right] .
\end{aligned}
$$

Assim,

$$
\bar{\rho} \rho_{c} \frac{U_{c} L_{c}}{\mu_{c}} \frac{D \overline{\mathbf{u}}}{D \bar{t}}=-\nabla^{\prime} \bar{p}+\nabla^{\prime}\left(\bar{\mu}\left(\nabla^{\prime} \overline{\mathbf{u}}+\nabla^{\prime} \overline{\mathbf{u}}^{T}\right)\right)+\frac{G \tau_{c}}{\mu_{c}} \nabla^{\prime} \cdot \overline{\mathbf{S}}_{p}+\frac{L_{c}^{2} \mathbf{f}}{\mu_{c} U_{c}}
$$

Definido os adimensionais $R e=\frac{\rho_{c} U_{c} L_{c}}{\mu_{c}}$ (número de Reynolds), $\beta=\frac{G \tau_{c}}{\mu_{c}}$ e $\overline{\mathbf{f}}=-\frac{L_{c}^{2} \mathbf{f}}{\mu_{c} U_{c}}=-\frac{L_{c} \tau_{c}}{\mu_{c}} \mathbf{f}$, ao substituir estes valores na equação anterior conseguimos a equação adimensional do movimento

$$
R e \bar{\rho} \frac{D \overline{\mathbf{u}}}{D \bar{t}}=-\nabla^{\prime} \bar{p}+\nabla^{\prime}\left(\bar{\mu}\left(\nabla^{\prime} \overline{\mathbf{u}}+\nabla^{\prime} \overline{\mathbf{u}}^{T}\right)\right)+\beta \nabla^{\prime} \cdot \overline{\mathbf{S}}_{p}-\overline{\mathbf{f}} .
$$

Da mesma forma, obtemos para a equação (A.2)

$$
\nabla^{\prime} \cdot \overline{\mathbf{u}}=0
$$

Para o tensor (A.4), a adimensionalização é como segue

$$
\begin{aligned}
& \tau\left(\frac{D \mathbf{S}_{p}}{D t}-\left(\nabla \mathbf{u} \mathbf{S}_{p}+\mathbf{S}_{p} \nabla \mathbf{u}^{T}\right)\right)=-\left(\mathbf{S}_{p}-G I\right) \\
& \tau\left(\frac{G}{t_{c}} \frac{D \overline{\mathbf{S}}_{p}}{D \bar{t}}-\left(\frac{G U_{c}}{L_{c}} \nabla^{\prime} \overline{\mathbf{u}} \overline{\mathbf{S}}_{p}+\frac{G U_{c}}{L_{c}} \overline{\mathbf{S}}_{p} \nabla^{\prime} \overline{\mathbf{u}}^{T}\right)\right)=-G\left(\overline{\mathbf{S}}_{p}-I\right) .
\end{aligned}
$$


Logo,

$$
\begin{aligned}
& \tau\left(\frac{1}{t_{c}} \frac{D \overline{\mathbf{S}}_{p}}{D \bar{t}}-\frac{U_{c}}{L_{c}}\left(\nabla^{\prime} \overline{\mathbf{u}} \overline{\mathbf{S}}_{p}+\overline{\mathbf{S}}_{p} \nabla^{\prime} \overline{\mathbf{u}}^{T}\right)\right)=-\left(\overline{\mathbf{S}}_{p}-I\right), \\
& \tau\left(\frac{1}{c} \frac{D \overline{\mathbf{S}_{p}}}{D \bar{t}}-\frac{1}{t_{c}}\left(\nabla^{\prime} \overline{\mathbf{u}} \overline{\mathbf{S}}_{p}+\overline{\mathbf{S}}_{p} \nabla^{\prime} \overline{\mathbf{u}}^{T}\right)\right)=-\left(\overline{\mathbf{S}}_{p}-I\right), \\
& \frac{\tau}{t_{c}}\left(\frac{D \overline{\mathbf{S}}_{p}}{D \bar{t}}-\left(\nabla^{\prime} \overline{\mathbf{u}} \overline{\mathbf{S}}_{p}+\overline{\mathbf{S}}_{p} \nabla^{\prime} \overline{\mathbf{u}}^{T}\right)\right)=-\left(\overline{\mathbf{S}}_{p}-I\right), \\
& W_{i} \overline{\mathbf{S}}_{p}^{\nabla}=-\left(\overline{\mathbf{S}}_{p}-I\right),
\end{aligned}
$$

onde $W_{i}=\frac{\tau}{t_{c}}$ é o número de Weissenberg, dado pela razão entre o tempo de relaxação polimérica com o tempo característico do escoamento do fluido. O valor $W_{i}$ é uma medida dos efeitos não newtonianos pois é o tempo relativo de relaxação ou do polímero (macromoléculas) ou de microestruturas no fluido. Se o tempo de relaxação polimérica $\tau$ é pequeno ao ser comparado com o tempo característico, a microestrutura relaxa rapidamente ao equilíbrio e o fluido tem um comportamento newtoniano, por outro lado se $\tau$ é comparável com o tempo característico, isto é, $W_{i}=O(1)$, a microestrutura não tem tempo para retornar ao equilíbrio, dando origem a efeitos não newtonianos no escoamento.

Para números de Reynolds pequenos $(R e \rightarrow 0)$ em (A.5)-(A.7), temos as equações Stokes-Oldroyd-B

$$
\begin{gathered}
-\nabla \bar{p}+\nabla\left(\bar{\mu}\left(\nabla \overline{\mathbf{u}}+\nabla \overline{\mathbf{u}}^{T}\right)\right)+\beta \nabla \cdot \overline{\mathbf{S}}_{p}=\overline{\mathbf{f}}, \\
\nabla \cdot \overline{\mathbf{u}}=0, \quad \text { em } \Omega, \\
W_{i} \overline{\mathbf{S}}_{p}^{\nabla}=-\left(\overline{\mathbf{S}}_{p}-I\right), \quad \text { em } \Omega .
\end{gathered}
$$

Notemos que se isolarmos a massa específica característica $\rho_{c}$ em (A.1), podemos obter uma equação adimensional diferente, a qual pode relacionar outras propriedades como o número de Mach ou também podemos encontrar o número de Reynolds em uma posição diferente como o denominador. 


\section{Apêndice B}

\section{Mapa de visibilidade para variáveis definidas no meio das arestas}

No Capítulo 4, definimos o mapa de visibilidade de variáveis definidas no centro das células em uma malha composta, o qual é necessário para compor a estrutura matricial de uma discretização em uma malha adaptativa do tipo SAMR.

Para o caso de variáveis centradas, empregamos no Capítulo 4 a malha da Figura 4.2(b) e mostramos para ela como fica o mapa de visibilidade. No presente apêndice, fazemos da mesma forma para variáveis definidas nas arestas $x$ e $y$ seus respetivos mapas de visibilidade. O mapa de visibilidade que mostramos para essa malha é para variáveis com condição de contorno de Dirichlet ou de Neumann. Neste apêndice também incluímos um exemplo do mapa de visibilidade para variáveis definidas no meio das arestas $x$ com condição de contorno periódica.

Como foi definido no Capítulo 4, o mapa de visibilidade é uma aplicação que retorna "1" quando a célula é visível e "0" quando é invisível. Ele é importante durante o processo de construção da matriz que representa a discretização da equação em malha composta.

Para decidir se uma aresta é visível ou invisível, temos que ter um cuidado adicional com relação ao caso quando a variável está definida no centro da célula. Retalhos de malha contidos em um mesmo nível podem compartilhar arestas (isto é, arestas que se tocam) e, ao mesmo tempo, podem não estar recobertos por nenhum retalho de malha em um nível mais fino.

Uma aresta compartilhada por retalhos de malha diferentes só pode ser representada uma única vez na matriz. Por isto, decidimos que para as arestas compartilhadas, somente uma delas poderá ser visível e as outras serão então invisíveis. A convenção que assumimos no caso de arestas compartilhadas é que sempre a aresta que pertence ao retalho à direita (correspondente ao retalho superior) comparando os retalhos será a visível. Isto pode ser visto no segundo nível da malha composta que estamos usando como exemplo na Figura 4.2(b), onde para variáveis definidas na aresta $x$, os retalhos $G_{2,1}$ e $G_{2,2}$ compartilham uma parte da aresta $x$.

Mostramos nas figuras B.1 e B.2 o mapa de visibilidade do nível base e do segundo nível de refinamento, respectivamente.

O valor do mapa de visibilidade das arestas compartilhadas na aresta $x$ do retalho à direita é 1 mas o valor destas arestas no retalho à esquerda é 0 (Figura B.2). 


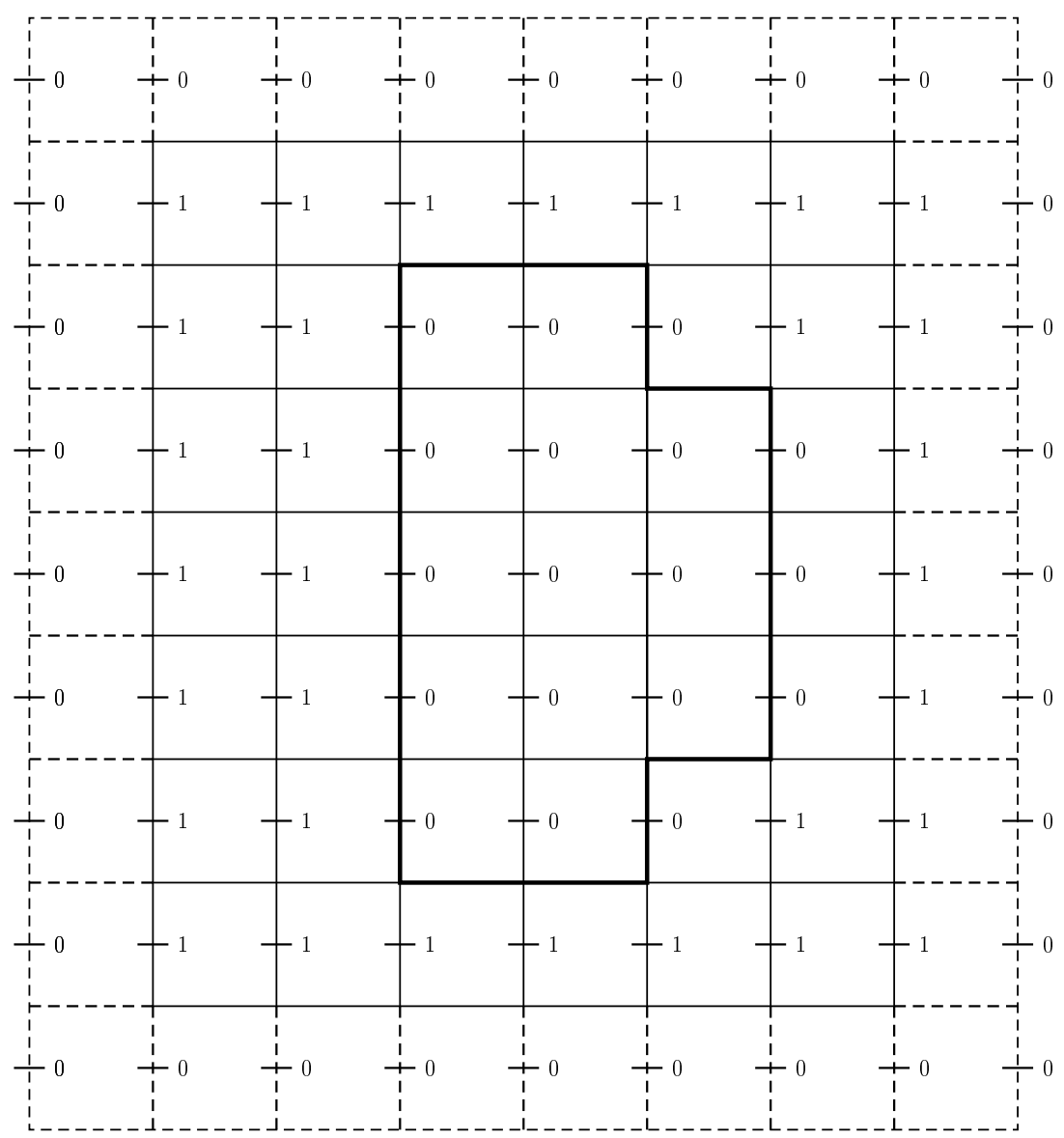

Figura B.1: Mapa de visibilidade nivel base na aresta $x$ para condição de contorno de Dirichlet ou de Neumann. 

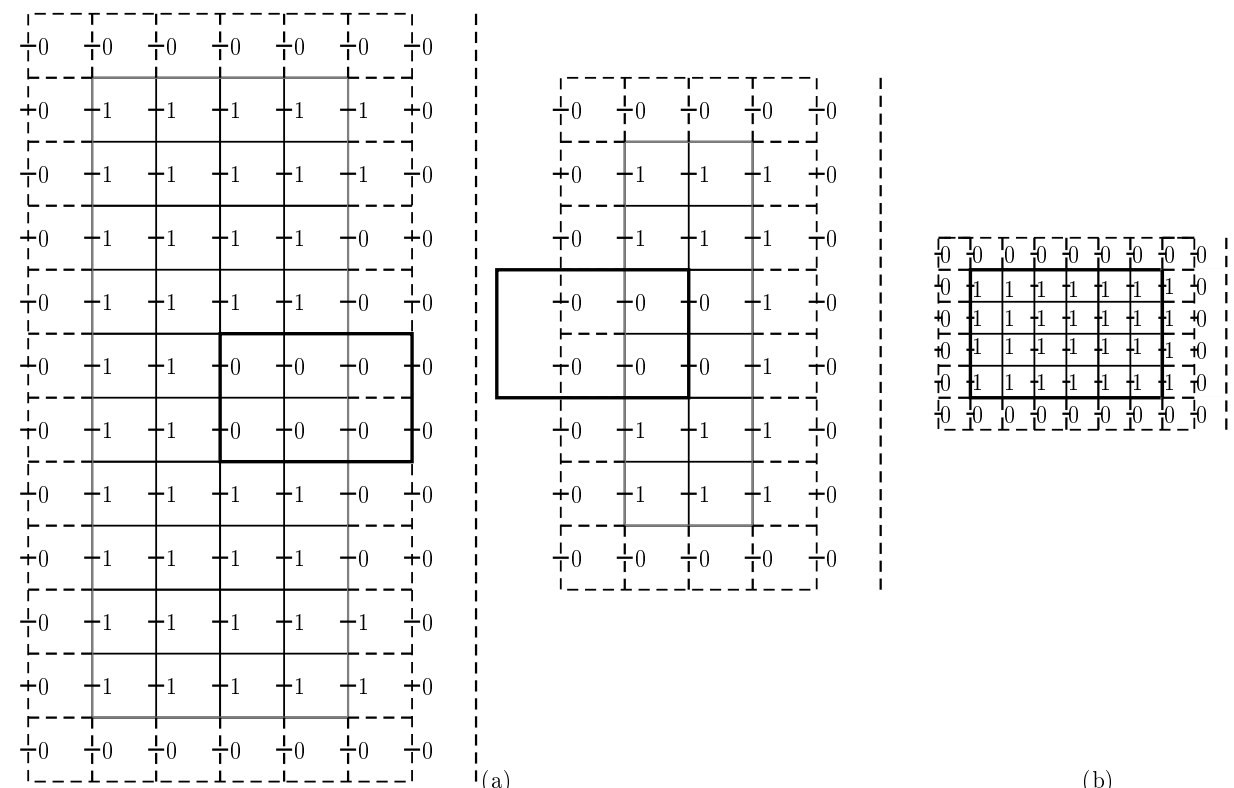

Figura B.2: Mapa de visibilidade na aresta $x$ para condição de contorno de Dirichlet ou de Neumann. (a) Nivel base $+1=L_{2}$. (b) Nivel base $+2=L_{3}$.

Finalmente, também temos nas figuras B.3 e B.4 o mapa de visibilidade para as variáveis definidas na aresta $y$. Notemos que neste caso as comparações de visibilidade correspondem claramente à aresta $y$.

A quantidade de células visíveis (total de "1's" no mapa de visibilidade) para as variáveis definidas na aresta $x$ é 113 e para as variáveis definidas na aresta $y$ é de 111. No próximo apêndice, o qual contém a enumeração correspondente aos mapas de visibilidade das figuras (B.1)-(B.2), podemos ver que a quantidade de células visíveis é o maior número possível da enumeração. 


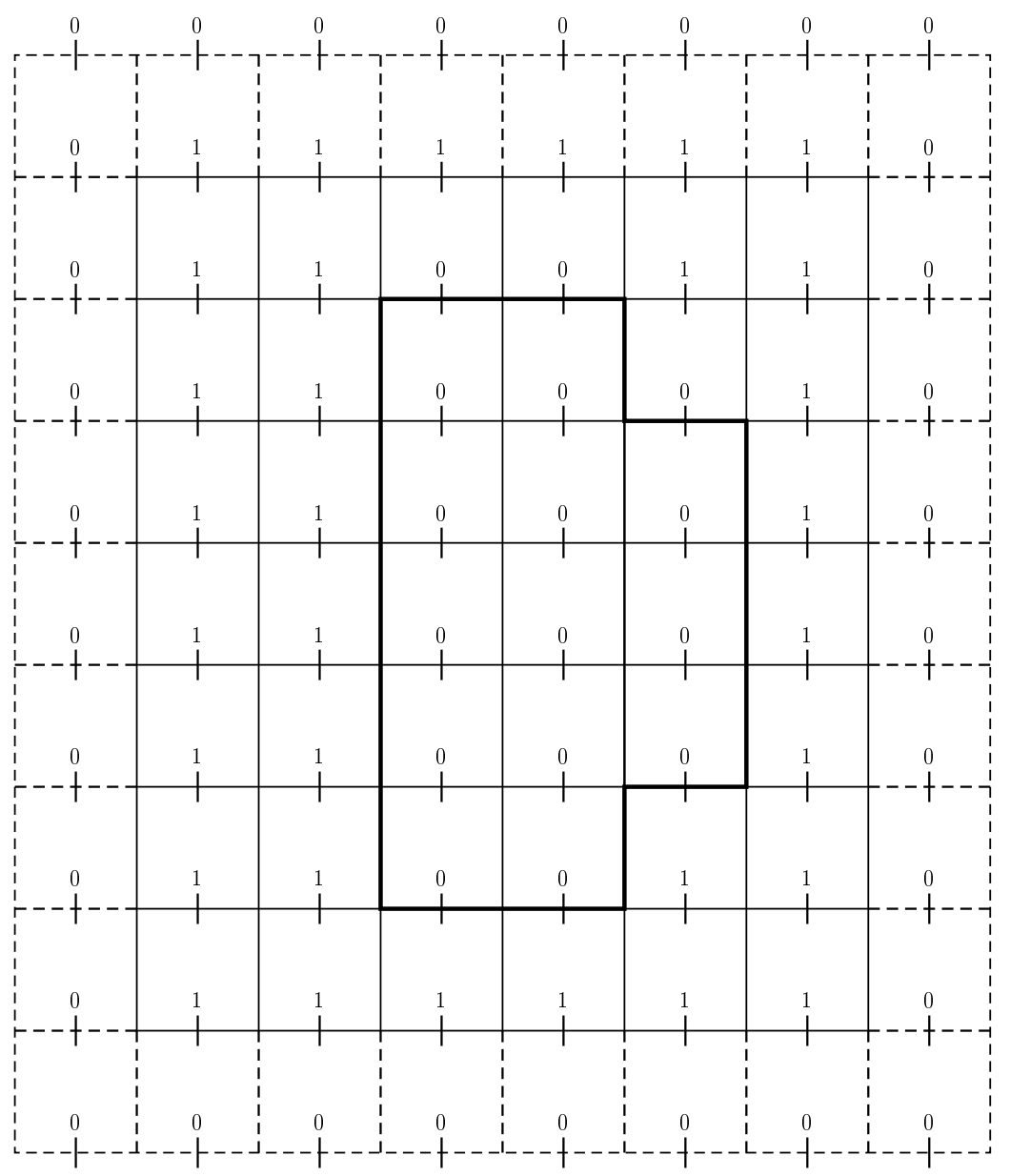

Figura B.3: Mapa de visibilidade nivel base na aresta y para condição de contorno de Dirichlet ou de Neumann. 

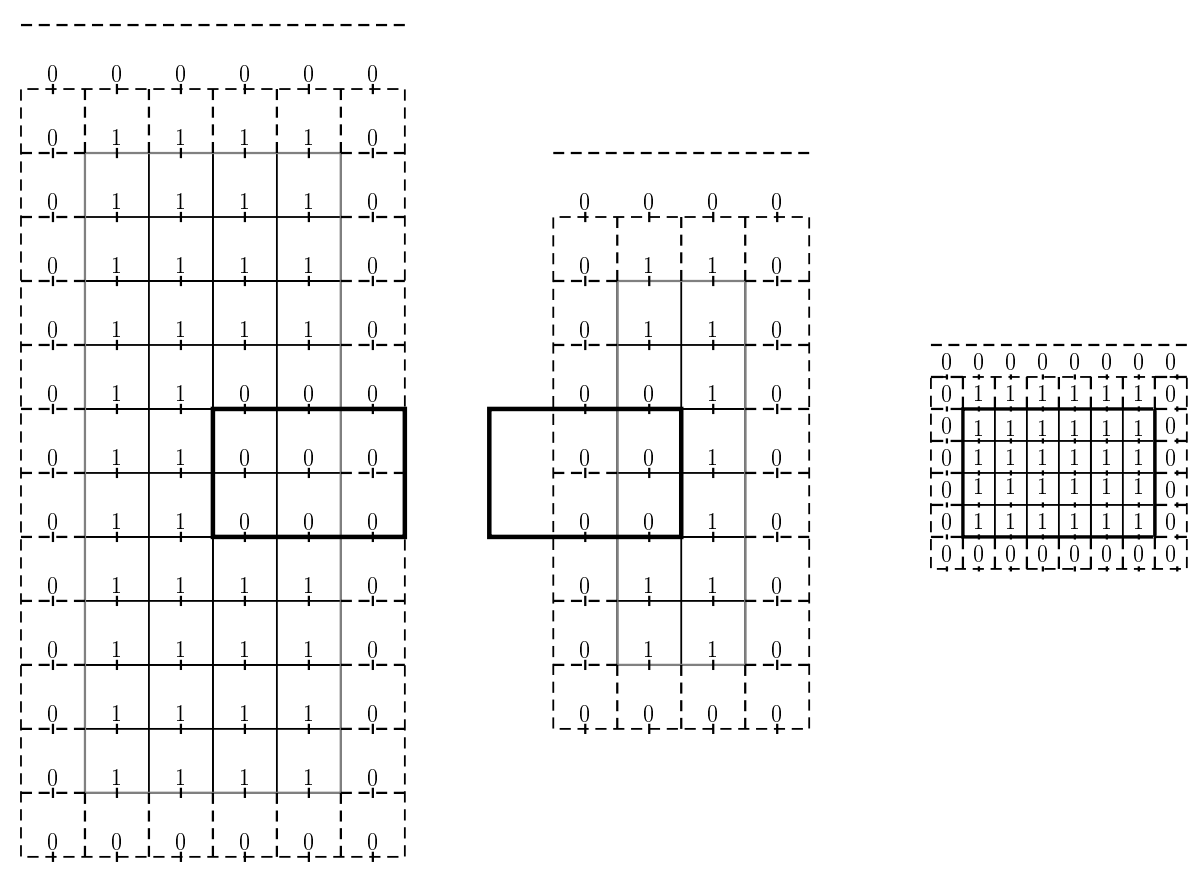

(a)

(b)

Figura B.4: Mapa de visibilidade na aresta $x$ para condição de contorno de Dirichlet ou de Neumann. (a) Nivel base $+1=L_{2}$. (b) Nivel base $+2=L_{3}$.

No caso periódico é importante lembrar que a aresta direita(superior) na fronteira do domínio físico é a mesma que a aresta esquerda(inferior) quando temos periodicidade na direção $x$ (direção $y$, respectivamente). Neste caso, deve-se tomar mais cuidado no momento de criar o mapa de visibilidade.

A convenção que assumimos para decidir qual das duas aresta é invisível é que as arestas que estão à direita(superior) na fronteira do domínio físico serão invisíveis. Por exemplo, para a malha na Figura B.5(a) com os retalhos ordenados como na Figura B.5(b), obtemos para a aresta $x$ periódica o mapa de visibilidade mostrado na Figura B.6. No mapa de visibilidade, observe na Figura B.6(a) como no nível base a aresta direita não tem células visíveis e na Figura B.6(b) correspondente ao segundo nível, como as arestas compartilhadas também influem na escolha das células visíveis.

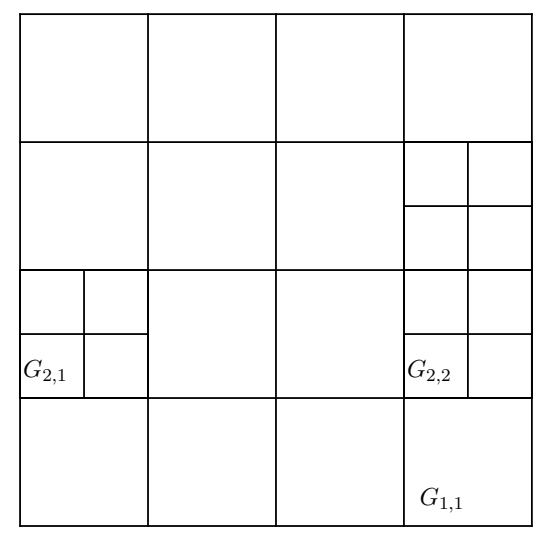

(a)

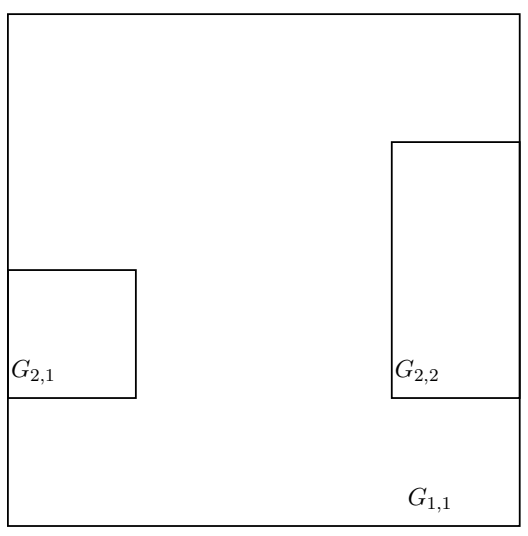

(b)

Figura B.5: (a) Malha composta com dois niveis para caso periódico. (b) Retalhos da malha composta. 


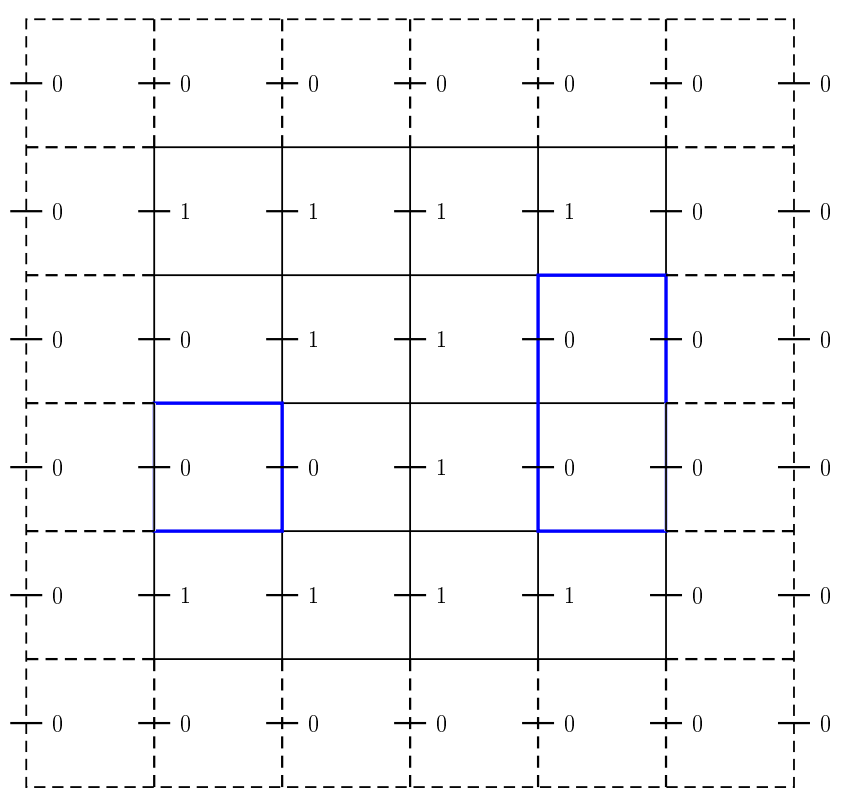

(a)

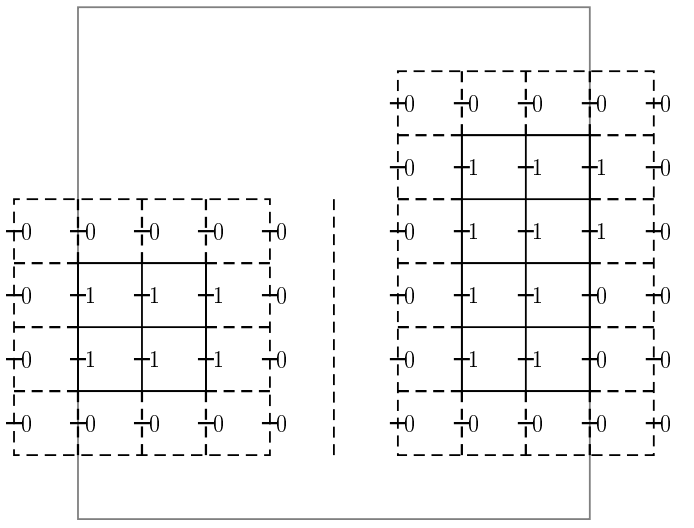

(b)

Figura B.6: Mapa de visibilidade na aresta $x$ com condição de contorno periódica na direção $x$. (a) Nível base. (b) Nivel base $+1=L_{2}$ aresta $x$. 


\section{Apêndice C}

\section{Enumeração para variáveis definidas no meio das arestas}

No Capítulo 4, definimos uma enumeração para variáveis definidas no centro das células em uma malha composta. Esta enumeração é usada para definir a estrutura matricial de uma discretização com a técnica AMR somente para células visíveis.

Para mostrar a enumeração no caso de variáveis centradas, usamos no Capítulo 4 a malha na Figura 4.2(b). No presente apêndice, mostramos da mesma forma para variáveis com condição de contorno de Dirichlet ou de Neumann definidas na aresta $x$ e na aresta $y$ suas respectivas enumerações. Também incluímos a enumeração para o caso periódico dado no Apêndice B.

Seguindo a mesma ideia de enumeração para variáveis definidas no centro das célula em forma consecutiva da esquerda à direita, seguindo a ordem dos retalhos em cada nível e iniciando desde o nível mais grosso até o mais fino, temos definida a enumeração para variáveis definidas na aresta $x$ e na aresta $y$. Apenas as posições visíveis recebem enumeração - desta forma, o uso do mapa de visibilidade (Apêndice B) é muito importante para que arestas compartilhadas tenham uma única enumeração.

Nas figuras C.1 e C.2 temos a enumeração do caso de exemplo para variáveis definidas no meio da aresta $x$ (ou da aresta $y$ ) para condição de contorno de Dirichlet e de Neumann. 


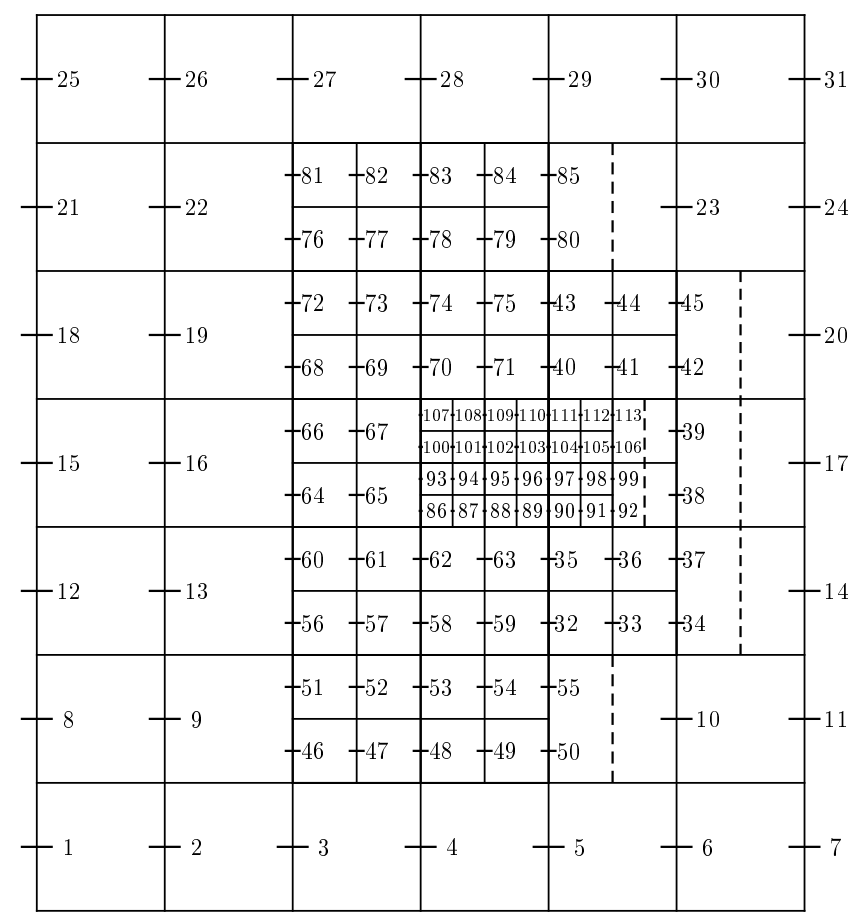

$$
\begin{aligned}
& 1 \\
& 1 \\
& 1 \\
& 1 \\
& 1 \\
& 1 \\
& 1 \\
& 1 \\
& 1 \\
& 1 \\
& 1 \\
& 1 \\
& 1 \\
& 1 \\
& 1 \\
& 1 \\
& 1 \\
& 1 \\
& 1
\end{aligned}
$$

Figura C.1: Enumeração na aresta $x$ para condições de contorno de Dirichlet ou de Neumann.

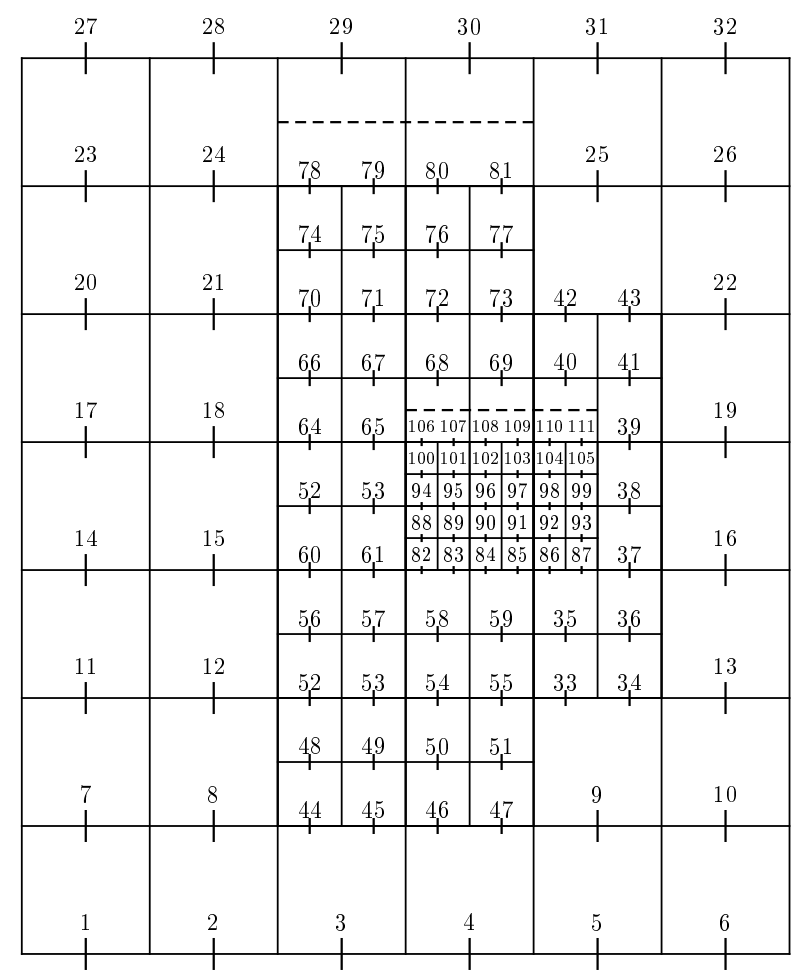

Figura C.2: Enumeração na aresta y para condições de contorno de Dirichlet ou de Neumann.

Para condições de contorno periódicas, como foi dito no Apêndice B o mapa de visibilidade é diferente do obtido com condições de contorno de Dirichlet ou de Neumann. Para ilustrar isto, seguindo o mapa de visibilidade na Figura B.6 obtido para um exemplo periódico na direção $x$ no Apêndice $\mathrm{B}$, obtemos a enumeração correspondente às células definidas na aresta $x$, mostrada na Figura C.3. 


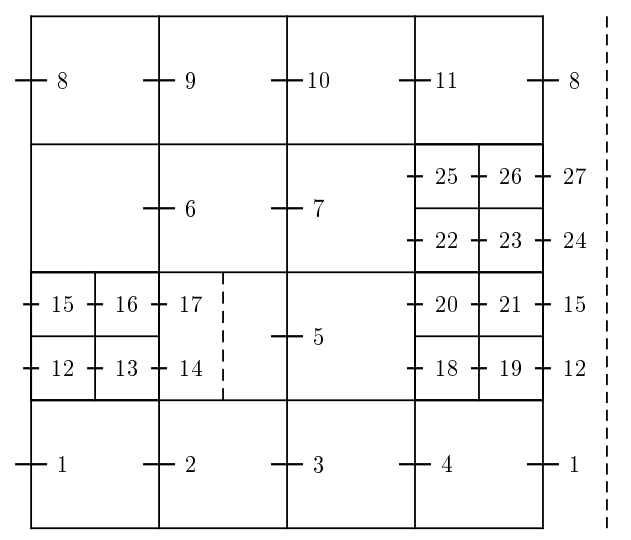

Figura C.3: Enumeração na aresta x para o caso periódico. 


\section{Referências Bibliográficas}

[1] M.J. Aftosmis, M.J. Berger, e S.M. Murman. Applications of space-filling curves to cartesian methods for CFD. Technical report, NAS, March 2004. 67

[2] M. Albers. A local mesh refinement multigrid method for 3-d convection problems with strongly variable viscosity. Journal of Computational Physics, 160(1):126 - 150, 2000. 5, 85

[3] Applied Numerical Algorithms Group (ANAG). Chombo - Software for Adaptive Solutions of Partial Differential Equations, 2013. 5, 53, 57, 61, 78

[4] K.J. Arrow, L. Hurwicz, e H. Uzawa. Studies in linear and non-linear programming. With contributions by H. B. Chenery, S. M. Johnson, S. Karlin, T. Marschak, R. M. Solow. Stanford Mathematical Studies in the Social Sciences, vol. II. Stanford University Press, Stanford, 1958. $5,42,43,77,86,123$

[5] U.M. Ascher, S.J. Ruuth, e B.T.R. Wetton. Implicit-explicit methods for time-dependent partial differential equations. SIAM J. Numer. Anal., 32(3):797-823, 1995. 23, 29, 30

[6] C. Auth e H. Harder. Multigrid solution of convection problems with strongly variable viscosity. Geophysical Journal International, 137(3):793-804, 1999. 5

[7] C. Bacuta. A unified approach for Uzawa Algorithms. SIAM J. Numerical Analysis, 44(6):2633-2649, 2006. 5, 77, 86, 123

[8] C. Bacuta, P.S. Vassilevski, e S. Zhang. A new approach for solving stokes systems arising from a distributive relaxation method. Numerical Methods for Partial Differential Equations, 27(4):898-914, 2011. 5, 77

[9] A. Baeza e P. Mulet. Adaptive mesh refinement techniques for high-order shock capturing schemes for multi-dimensional hydrodynamic simulations. International Journal for Numerical Methods in Fluids, 52(4):455-471, 2006. 23, 52

[10] S. Balay, J. Brown, K. Buschelman, V. Eijkhout, W.D. Gropp, D. Kaushik, M.G. Knepley, L.C. McInnes, B.F. Smith, e H. Zhang. PETSc users manual. Technical Report ANL-95/11 - Revision 3.3, Argonne National Laboratory, 2012. 78

[11] S. Balay, J. Brown, K. Buschelman, W.D. Gropp, D. Kaushik, M.G. Knepley, L.C. McInnes, B.F. Smith, e H. Zhang. PETSc Web page, 2012. http://www.mcs.anl.gov/petsc. 53, 71, 78, 123

[12] S. Balay, W.D. Gropp, L.C. McInnes, e B.F. Smith. Efficient management of parallelism in object oriented numerical software libraries. In E. Arge, A. M. Bruaset, e H. P. Langtangen, editors, Modern Software Tools in Scientific Computing, pages 163-202. Birkhäuser Press, 1997. 78

[13] R. Barrett, M. Berry, T.F. Chan, J. Demmel, J. Donato, J. Dongarra, V. Eijkhout, R. Pozo, C. Romine, e H. Van der Vorst. Templates for the solution of linear systems: building blocks for iterative methods. Society for Industrial and Applied Mathematics (SIAM), Philadelphia, PA, 1994. 5, 43, 123 
[14] J.B. Bell, P. Colella, e H.M. Glaz. A second-order projection method for the incompressible Navier-Stokes equations. Journal of Computational Physics, 85(2):257-283, 1989. 29

[15] J.B. Bell, L.H. Howell, e P. Colella. An efficient second-order projection method for viscous incompressible flow. In A. D. Vakili e C. Gauthier, editors, Space Manufacturing 8 - Energy and Materials from Space, pages 24-26, April 1991. 52

[16] M.J. Berger e P. Colella. Local adaptive mesh refinement for shock hydrodynamics. J. Comput. Phys., 82:64-84, May 1989. 3, 52, 53, 54

[17] M.J. Berger e J. Oliger. Adaptive mesh refinement for hyperbolic partial differential equations. Journal of Computational Physics, 53(3):484 - 512, 1984. 3, 52, 54

[18] M.J. Berger e I. Rigoutsos. An algorithm for point clustering and grid generation. IEEE Transactions on Systems, Man, and Cybernetics, 21:1278-1286, 1991. 3, 52, 54

[19] R.B. Bird, P.J. Dotson, e N.L. Johnson. Polymer solution rheology based on a finitely extensible bead-spring chain model. Journal of Non-Newtonian Fluid Mechanics, 7(2):213-235, 1980. $2,17,23,41$

[20] R.H. Bisseling e W. Meesen. Communication balancing in parallel sparse matrix-vector multiplication. ETNA. Electronic Transactions on Numerical Analysis [electronic only], 21:47-65, 2005. 85

[21] D. Braess e R. Sarazin. An efficient smoother for the stokes problem. Applied Numerical Mathematics, 23(1):3 - 19, 1997. < ce:title>Multilevel Methods $</$ ce:title>. 5, 77

[22] J.H. Bramble, J.E. Pasciak, e A. T. Vassilev. Uzawa type algorithms for nonsymmetric saddle point problems. Math. Comp., 69(230):667-689, 2000. 5

[23] R.L. Burden e J.D. Faires. Numerical analysis. Brooks-Cole, Cengage Learning, Boston, MA, USA, nineth edition, 2010. 5, 8, 37, 40

[24] J.C. Butcher. Numerical methods for ordinary differential equations. John Wiley \& Sons Ltd., Chichester, 2003. 23, 39, 40

[25] P.C. Calegari. Simulação computacional de escoamentos reativos com baixo número Mach aplicando técnicas de refinamento adaptativo de malhas. Tese de Doutorado, Universidade de São Paulo, São Paulo, Brasil, 2012. 29, 52, 81

[26] H.D. Ceniceros e J.E. Fisher. A fast, robust, and non-stiff immersed boundary method. J. Comput. Physics, 230(12):5133-5153, 2011. 124

[27] H.D. Ceniceros e J.E. Fisher. Peristaltic pumping of a viscoelastic fluid at high occlusion ratios and large weissenberg numbers. Journal of Non-Newtonian Fluid Mechanics, 171-172(0):31 41, 2012. 2, 39, 124

[28] H.D. Ceniceros, J.E. Fisher, e A.M Roma. Efficient solutions to robust, semi-implicit discretizations of the immersed boundary method. J. Comput. Physics, 228(19):7137-7158, 2009. 124

[29] H.D. Ceniceros, R.L. Nós, e A.M. Roma. Three-dimensional, fully adaptive simulations of phase-field fluid models. Journal of Computational Physics, 229(17):6135 - 6155, 2010. 5, 31, 52,60

[30] H.D. Ceniceros e A.M. Roma. Study of the long-time dynamics of a viscous vortex sheet with a fully adaptive nonstiff method. Physics of Fluids, 16(12):4285 - 4318, 2004. 27, 34 
[31] H.D. Ceniceros e A.M. Roma. A multi-phase flow method with a fast, geometry-based fluid indicator. Journal of Computational Physics, 205(2):391-400, 2005. 4, 12, 13, 27, 28, 29, 47

[32] H.D. Ceniceros, A.M. Roma, A. Silveira-Neto, e M.M. Villar. A robust, fully adaptive hybrid level-set/front-tracking method for two-phase flows with an accurate surface tension computation. Communications in Computational Physics, 2010. 3, 4, 5, 7, 8, 11, 12, 13, 14, 23, 27, $29,34,52,53,57,60,123$

[33] Center for Applied Scientific Computing. SAMRAI - Structured Adaptive Mesh Refinement Application Infrastructure, 2013. 53, 57, 61, 78

[34] A.J. Chorin. Numerical solution of the Navier-Stokes equations. Math. Comp., 22:745-762, 1968. $6,23,29,123$

[35] A.J. Chorin e J.E. Marsden. A Mathematical Introduction to Fluid Mechanics. SpringerVerlag, New York NY, 1993. 23, 30

[36] M.-R. Cui. Analysis of iterative algorithms of uzawa type for saddle point problems. Applied Numerical Mathematics, 50(2):133-146, 2004. 5, 43

[37] T de Pinheiro Macedo. Gts-cpt: Uma implementação de função indicadora utilizando a transformada do ponto mais próximo. Trabalho de Conclusão de Curso (Graduação em Bacharelado em Matemática Aplicada)- IME-USP, 2013. 29

[38] F. Deserno. Basic Optimisation Strategies for CFD-Codes. Technical report, FriedrichAlexander-Universität Erlangen-Nürnberg, 2003. 99

[39] J. Douglas e T. Dupont. Alternating-direction Galerkin methods on rectangles. Numerical Solution of Partial Differential Equations, II (SYNSPADE 1970), pages 133-214, 1971. 31

[40] H.C. Elman. Multigrid and krylov subspace methods for the discrete stokes equations. Int. J. Numer. Meth. Fluids, 22:755-770, 1994. 5, 23, 42, 86

[41] R.E. Ewing, R.D. Lazarov, e P. S. Vassilevski. Local refinement techniques for elliptic problems on cell-centered grids; II. optimal order two-grid iterative methods. Numerical Linear Algebra with Applications, 1(4):337-368, 1994. 6, 60, 78

[42] R.E. Ewing, R.D. Lazarov, e P.S. Vassilevski. Local refinement techniques for elliptic problems on cell-centered grids. I. Error analysis. Math. Comp., 56(194):437-461, 1991. 6, 33, 58, 60, 78

[43] R. Fattal e R. Kupferman. Time-dependent simulation of viscoelastic flows at high Weissenberg number using the log-conformation representation. Journal of Non-Newtonian Fluid Mechanics, 126(1):23 - 37, 2005. 2, 3, 40

[44] L.J. Fauci e R. Dillon. Biofluidmechanics of reproduction. Annual Review of Fluid Mechanics, 38:371-394, 2006. 2, 4

[45] J.H. Ferziger e M. Perić. Computational methods for fluid dynamics, volume 2. Springer Berlin, 1999. 29

[46] D. Gottlieb e S.A. Orszag. Numerical analysis of spectral methods: theory and applications, volume 26. Society for industrial and applied mathematics, 1993. 31

[47] B. E. Griffith, R. D. Hornung, D. M. McQueen, e C.S. Peskin. An adaptive, formally second order accurate version of the immersed boundary method. J. Comput. Phys., 223(1):10-49, April 2007. 52 
[48] B.E. Griffith. Simulating the blood-muscle-valve mechanics of the heart by an adaptive and parallel version of the immersed boundary method. Tese de Doutorado, New York University, New York, Estados Unidos, 2005. 29, 52, 123

[49] B. Gustafsson e J. Nilsson. Boundary conditions and estimates for the steady stokes equations on staggered grids. J. Sci. Comput., 15(1):29-59, March 2000. 5

[50] F.H. Harlow e J.E. Welch. Numerical calculation of time-dependent viscous incompressible flow of fluid with a free surface. Physics of Fluid, pages 251-263, 1965. 24

[51] A. Harten. High resolution schemes for hyperbolic conservation laws. Journal of computational physics, 49(3):357-393, 1983. 3, 6, 39

[52] E.J Hinch. Non-newtonian geophysical fluid dynamics : Lectures by john hinch. volume 2003. Woods Hole Oceanographic Institution, 2003. 3

[53] X. Huang e X. Zhang. Adaptive mesh refinement for computational aeroacoustics. In 11th AIAA/CEAS Aeroacoustics Conference. American Institute of Aeronautics and Astronautics, May 2005. AIAA 2005-2873. 6, 78

[54] M.A. Hulsen, R. Fattal, e R. Kupferman. Flow of viscoelastic fluids past a cylinder at high weissenberg number: stabilized simulations using matrix logarithms. J. Non-Newtonian Fluid Mech., 127(1):27-39, 2005. 3, 8, 40

[55] J. Kim e P. Moin. Application of a fractional-step method to incompressible Navier-Stokes equations. Journal of Computational Physics, 59(2):308-323, 1985. 29

[56] S.D. Kim. Uzawa algorithms for coupled Stokes equations from the optimal control problem. Calcolo, 46(1):37-47, 2009. 5, 23, 42, 77, 86, 123

[57] D.H. Klein, C. Garcia-Cervera, H.D. Ceniceros, e L.G. Leal. Computational studies of the shear flow behaviour of a model for nematic liquid crystalline polymers. In Rob May e A. J. Roberts, editors, Proc. of 12th Computational Techniques and Applications Conference CTAC-2004, volume 46, pages C210-C244, April 2005. 4, 5, 43, 44, 77, 86, 123

[58] D.H. Klein, L.G. Lealy, C.J. García-Cervera, e H.D. Ceniceros. Three-dimensional sheardriven dynamics of polydomain textures and disclination loops in liquid crystalline polymers. Journal of Rheology, 52, 2008. 23, 42, 43

[59] C. Kreuzer. Analysis of an adaptive Uzawa finite element method for the nonlinear Stokes problem. Math. Comp., 81(277):21-55, 2012. 5, 23, 42, 86

[60] R.G. Larson. The Structure and Rheology of Complex Fluids. Oxford University Press, USA, November 1998. 2, 3, 16, 23

[61] J. Leung, L. Kelly, e J.H. Anderson. Handbook of Scheduling: Algorithms, Models, and Performance Analysis. CRC Press, Inc., Boca Raton, FL, USA, 2004. 85

[62] Z. Li e K. Ito. The Immersed Interface Method: Numerical Solutions of PDEs Involving Interfaces and Irregular Domains. Society for Industrial and Applied Mathematics, 2006. 27

[63] A.L.F. Lima e Silva. Desenvolvimento e Implementação de Uma Nova Metodologia para Modelagem de Escoamentos sobre Geometrias Complexas: Método da fronteira Imersa com Modelo Físico Virtual. Tese de Doutorado, Universidade Federal de Uberlândia, Uberlândia, Brasil,, 2002. 26

[64] D. Martin e K. Cartwright. Solving poisson's equation using adaptive mesh refinement, 1996. $5,58,92$ 
[65] D.F. Martin. An adaptive cell-centered projection method for the incompressible Euler equations. Tese de Doutorado, University of California at Berkeley, 1998. 6, 33, 77, 87

[66] S. Mauch. A fast algorithm for computing the closest point and distance transform. Technical report, CALTECH ASCI, 2000. 28

[67] S. McKee, M.F. Tomé, V.G. Ferreira, J.A. Cuminato, A. Castelo, F.S. Sousa, e N. Mangiavacchi. The MAC method. Computers \&3 Fluids, 37(8):907 - 930, 2008. 24

[68] R. Mittal e G. Iaccarino. Immersed boundary methods. Annual Review of Fluid Mechanics, 37:239-261, 2005. 14

[69] L.-N. Moresi e V.S. Solomatov. Numerical investigations of 2D convection with extremely large viscosity variations, 1995. 5, 43, 86

[70] A. Niestegge e K. Witsch. Analysis of a multigrid strokes solver. Applied Mathematics and Computation, 35(3):291 - 303, 1990. 5

[71] R.L. Nós. Simulações de escoamentos bifásicos tridimensionais empregando métodos adaptativos e modelos de campo de fase. Tese de Doutorado, Universidade de São Paulo, São Paulo, Brasil, 2007. 7, 33, 53, 57, 63

[72] C.W. Oosterlee e F.J. Gaspar Lorenz. Multigrid methods for the stokes system. Computing in Science and Engineering, 8(6):34-43, 2006. 5, 77, 85

[73] S. Osher e R. Fedkiw. Level set methods and dynamic implicit surfaces, volume 153 of Applied Mathematical Sciences. Springer-Verlag, New York, 2003. 4, 6, 12, 13, 23, 27, 29, 33, 37

[74] S. Osher e R.P. Fedkiw. Level set methods: An overview and some recent results. Journal of Computational Physics, 169(2):463-502, 2001. 4, 15, 27

[75] R.G. Owens e T.N. Phillips. Computational rheology. Imperial College Press, London, 2002. 3

[76] S.V. Patankar. Numerical Heat Transfer and Fluid Flow. Hemisphere, Washington,D.C, 1980. 77,86

[77] S.V. Patankar e D.B. Spalding. A calculation procedure for heat, mass and momentum transfer in three-dimensional parabolic flows. International Journal of Heat and Mass Transfer, 15(10):1787 - 1806, 1972. 5

[78] G. S.H. Pau, J.B. Bell, A.S. Almgren, K. M. Fagnan, e M.J. Lijewski. An adaptive mesh refinement algorithm for compressible two-phase flow in porous media. Computational Geosciences, 16(3):577-592, 2012. 4, 27, 52

[79] M. Pernice e B.T. Gunney. Solution of the Modified Bratu Problem in SAMRAI. Technical report, Lawrence Livermore National Lab., CA., 2004. 52, 53, 57, 61, 78

[80] M. Pernice e D. Hornung. Newton-Krylov-FAC methods for problems discretized on locally refined grids. Comput. Vis. Sci., 8(2):107-118, April 2005. 33, 53, 61

[81] C. Peskin. Numerical analysis of blood flow in the heart. Journal of Computational Physics, 25:220-252, 1977. 4, 8, 14, 27, 29, 33, 123

[82] L.A. Petri. Métodos de fronteira imersa em mecânica dos fluidos. Disertação de Mestrado, Universidade de São Paulo, São Carlos, Brasil, 2010. 33, 86, 105, 110

[83] A. Pletzer, B. Jamroz, R. Crockett, e S. Sides. Compact cell-centered discretization stencils at fine-coarse block structured grid interfaces. Preprint submitted to Journal of Computational Physics, 2013. 6, 58, 60, 78 
[84] S. Popinet e S. Zaleski. A front-tracking algorithm for accurate representation of surface tension. International Journal for Numerical Methods in Fluids, 30:775-793, 1999. 27

[85] A.M. Roma. A multilevel self adaptive version of the immersed boundary method. Tese de Doutorado, New York University, New York, Estados Unidos, 1996. 6, 14, 24, 29, 33, 55, 60, $77,123,124$

[86] A.M. Roma, C.S. Peskin, e M.J. Berger. An adaptive version of the immersed boundary method. Journal of Computational Physics, 153(2):509 - 534, 1999. 52

[87] C.M. Rúa-Alvarez. Implementación de una librería orientada a objeto para matrices esparcidas en paralelo. Disertação de Mestrado, Universidad de Puerto Rico, Recinto Universitario de Mayagüez, Puerto Rico, outubro 2007. 85

[88] Y. Saad. Iterative Methods for Sparse Linear Systems, Second Edition. Society for Industrial and Applied Mathematics, 2 edition, April 2003. 33, 51, 61, 71, 85, 88

[89] R. Sedgewick. Algorithms in C, Parts 1-4: Fundamentals, Data Structures, Sorting, Searching. Addison Wesley, 3rd edition, December 1997. 61, 62, 69, 85

[90] R. Sene de Lima. Desenvolvimento e implementação de malhas adaptativas bloco-estruturadas para computação paralela em mecânica dos fluidos. Tese de Doutorado, Universidade Federal de Uberlândia, Uberlândia, Brasil, 2012. 7, 85

[91] C. Shen, J-M. Qiu, e A. Christlieb. Adaptive mesh refinement based on high order finite difference WENO scheme for multi-scale simulations. Journal of Computational Physics, $230(10): 3780-3802,2011.23$

[92] S. Shin, S.I. Abdel-Khalik, V. Daru, e D. Juric. Accurate representation of surface tension using the level contour reconstruction method. J. Comput. Phys., 203(2):493-516, March 2005. $4,12,14,15,27,29,35,47$

[93] C.W. Shu e S. Osher. Efficient implementation of essentially non-oscillatory shock-capturing schemes, II. Journal of Computational Physics, 83(1):32 - 78, 1989. 3, 6, 23, 37, 39

[94] O. Stanley e J.A. Sethian. Fronts propagating with curvature dependent speed: Algorithms based on hamilton-jacobi formulations. Journal of Computational Physics, 79(1):12-49, 1988. 4,12

[95] J. Stoer e R. Bulirsch. Introduction to numerical analysis, volume 12. Springer Verlag, 2002. 8,76

[96] J.C. Strikwerda. Finite difference schemes and partial differential equations. Society for Industrial and Applied Mathematics (SIAM), Philadelphia, PA, second edition, 2004. 36, 37, 40, 92

[97] R. Temam. Une méthode d'approximation de la solution des équations de Navier-Stokes. Bull. Soc. Math. France, 98:115-152, 1968. 6, 23, 29, 123

[98] J. Teran, L. Fauci, e Shelley M. Viscoelastic fluid response can increase the speed and efficiency of a free swimmer. Phys. Rev. Lett., 104:038101, Jan 2010. 4

[99] J. Teran, L. Fauci, e M. Shelley. Peristaltic pumping and irreversibility of a Stokesian viscoelastic fluid. Physics of Fluids, 20(7), 2008. 2, 4, 16, 23, 29, 36

[100] B. Thomases e M. Shelley. Emergence of Singular Structures in Oldroyd-B Fluids. Physics of Fluids, 19(10), 2007. 2, 4, 16, 36, 41 
[101] B. Thomases e M. Shelley. Transition to mixing and oscillations in a stokesian viscoelastic flow. Physical Review Letters, 103, 2009. 4, 16, 41

[102] M.C Thompson e J.H. Ferziger. An adaptive multigrid technique for the incompressible Navier-Stokes equations . Journal of Computational Physics, 82(1):94 - 121, 1989. 6, 33, 60, 77

[103] M.F. Tomé, A. Castelo, F.M. Federson, e A. Cuminato. A numerical method for solving the Oldroyd-B model for 3D free surface flows. Mecánica Computacional, XXIII, 2004. 16, 23, 24,36

[104] U. Trottenberg, C.W. Oosterlee, e A. Schuller. Multigrid. Academic Press, Inc., Orlando, FL, USA, 2001. 5, 33, 60, 86

[105] G. Tryggvason. Numerical simulations of the Rayleigh-Taylor instability. J. Comput. Phys., 75(2):253-282, April 1988. 11, 12, 14, 123

[106] G. Tryggvason, B. Bunner, A. Esmaeeli, D. Juric, N. Al-Rawahi, W. Tauber, J. Han, S. Nas, e Y.-J. Jan. A front-tracking method for the computations of multiphase flow. Journal of Computational Physics, 169(2):708-759, 2001. 4, 11, 12, 13, 14, 27, 29, 123

[107] S.O. Unverdi e G. Tryggvason. A front-tracking method for viscous, incompressible, multifluid flows. J. Comput. Phys., 100(1):25-37, May 1992. 4, 11, 12, 13, 14, 27, 29, 123

[108] H.A. van der Vorst. Bi-CGSTAB: A Fast and Smoothly Converging Variant of Bi-CG for the Solution of Nonsymmetric Linear Systems. Siam Journal on Scientific and Statistical Computing, 13, 1992. 5, 43, 123

[109] B. Van Straalen, P. Colella, D.T. Graves, e N. Keen. Petascale block-structured amr applications without distributed meta-data. In Proceedings of the 17th international conference on Parallel processing - Volume Part II, Euro-Par'11, pages 377-386, Berlin, Heidelberg, 2011. Springer-Verlag. 53, 57, 61, 78

[110] M.M. Villar. AMR2D Software. Universidade Federal de Uberlândia e Universidade de São Paulo, 2007. 33, 35, 124

[111] M.M. Villar. Análise numérica detalhada de escoamentos multifásicos bidimensionais. Tese de Doutorado, Universidade Federal de Uberlândia, Uberlândia, Brasil, 2007. 3, 4, 6, 7, 26, $27,29,33,53,57,60,63,64,86,92,95,101,117,124$

[112] D. Wang e S.J. Ruuth. Variable step-size implicit-explicit linear multistep methods for timedependent partial differential equations. J. Comput. Math., 26(6):838-855, 2008. 23, 29, 30, 31

[113] H. Wang, J. Kearney, e K. Atkinson. Arc-length parameterized spline curves for real-time simulation. In in Proc. 5th International Conference on Curves and Surfaces, pages 387-396, 2002. 27

[114] G. Wittum. Multi-grid methods for Stokes and Navier-Stokes equations. Numerische Mathematik, 54:543-563, 1989. 5, 77

[115] G. Wittum. On the convergence of multi-grid methods with transforming smoothers. Numerische Mathematik, 57:15-38, 1990. 5, 77

[116] X. Yang, X. Zhang, Z. Li, e Guo-Wei He. A smoothing technique for discrete delta functions with application to immersed boundary method in moving boundary simulations. J. Comput. Phys., 228:7821-7836, November 2009. 33 
[117] J.H. Yun. Variants of the Uzawa method for saddle point problem. Computers \& Mathematics with Applications, 65(7):1037-1046, 2013. 5, 43, 77

[118] J. Zhang e J. Shang. A class of Uzawa-SOR methods for saddle point problems. Applied Mathematics and Computation, 216(7):2163-2168, 2010. 5, 43 


\section{Índice Remissivo}

Algoritmo

SAMR Método de Uzawa com BiCG-Stab retalho-a-retalho, 73

algoritmo

BICG Stab, xxi, 44

AMR2D, 53

AMR3D, 53

célula

fantasma, 55

invisível, 51, 56, 63

recoberta, 55

visível, 51,55

condições de contorno, 55

CSR, 51, 61

derivada

convectada superior, 16

domínio

euleriano, 12, 52

lagrangiano, 12

equação

da continuidade, 11

da quantidade de movimento linear, 11

de Stokes estacionária, 20

equações

de Navier-Stokes, 11

escoamento

incompressível, 11

estacionário, 22

transiente, 21

esquema

ENO, 3

extrapolação de Richardson, 53

extrapolação-interpolação cúbica, 56

fase

contínua, 12

dispersa, 12

fluido

bifásico, 11, 13, 21, 22

não newtoniano, 12, 16

newtoniano, 12, 16 fluidos

não newtonianos, 3,7

newtonianos, 3,7

força

interfacial, 21

formulação

clássica, 14

híbrida, 14, 15

função

de Heaviside, 13, 15

indicadora

de fluidos, 12

indicadora de fluidos, 12

lista

ligada, 51

Método

da Projeção, 29

método

de Uzawa, 51

BiCG-Stab, 51

da Fronteira Imersa, 14, 52

de Acompanhamento de Fronteira, 13

de elementos finitos, 5

de Euler, 37

de fronteira imersa, 14

de Gauss-Seidel, 60

preto-vermelho, 5

de Runge-Kutta, 40

de Runge-Kutta TVD, 3

de Uzawa, 5

dos gradientes biconjugados estabilizado, 5

ENO, 40

híbrido

front-tracking/front-capturing, 11

multinível-multigrid, 5, 57, 60 malha

bloco estruturada, 3,52

composta, 52, 54

trivial, 70

mapa, 51

binário, 63 
de visibilidade, 63,64

de visibilidade de arestas, 63

modelo

FENE, 2

FENE-P, 2, 11, 17, 18, 20, 22

Oldroyd-B, 2, 11, 16-18, 20, 22

número

de Reynolds, 11, 20

de Weissenberg, 20, 40

nível

base, 55

Navier-Stokes, 3

retalhos (patches), 52

rotina

Average Var, 59

Calc_Ghost, 59

correct_Definevar, 60

FillSister_Face, 59

SAMRAI, 52

T-junctions, 57

técnica

AMR, 3, 45, 52

SAMR, 3, 5, 52, 71

TVD (Total Variation Diminishing), 39

upwinding, 37 